\title{
Visible-Light-Mediated Decarboxylative Tandem Carbocyclization of Acrylamide-attached Alkylidenecyclopropanes: Access to Polycyclic Benzazepine Derivatives
}

\author{
Xiao-Yu Zhang, ${ }^{a}$ Chao Ning, ${ }^{\text {a }}$ Yong-Jie Long, ${ }^{\text {a }}$ Yin Wei ${ }^{\mathrm{b}}$ and Min Shi ${ }^{* a, b}$ \\ ${ }^{a}$ Laboratory for Advanced Materials \& Institute of Fine Chemicals, School of Chemistry \& \\ Molecular Engineering, East China University of Science and Technology, 130 Meilong Road, \\ Shanghai 200237, P. R. China, ${ }^{b}$ State Key Laboratory of Organometallic Chemistry, Center for \\ Excellence in Molecular Synthesis, University of Chinese Academy of Sciences, Shanghai Institute \\ of Organic Chemistry, Chinese Academy of Sciences, 345 Lingling Road, Shanghai 200032, P. R. \\ China.rmshi@mail.sioc.ac.cn
}

\section{CONTENTS}

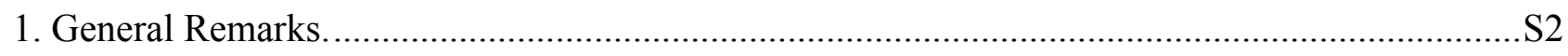

2. General Procedure for the Synthesis of Substrates.......................................................................S3

3. General Procedure for the Synthesis of Products..................................................................S4

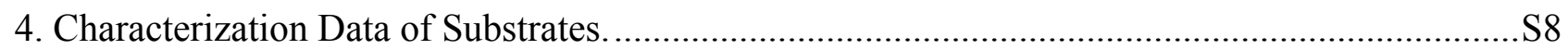

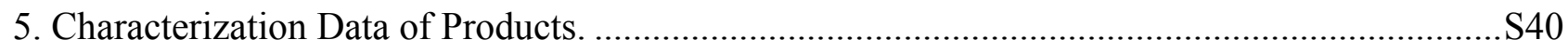

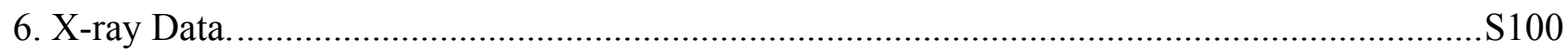

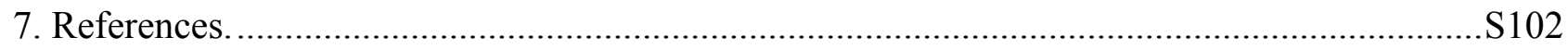




\section{General Remarks.}

Melting points were determined on a digital melting point apparatus and temperatures were uncorrected. NMR spectra were recorded with a Bruker spectrometer at $400 \mathrm{MHz}\left({ }^{1} \mathrm{H}\right.$ NMR), 100 $\mathrm{MHz}, 125 \mathrm{MHz}\left({ }^{13} \mathrm{C} \mathrm{NMR}\right)$ and $376 \mathrm{MHz}\left({ }^{19} \mathrm{~F} \mathrm{NMR}\right)$ in $\mathrm{CDCl}_{3}$, respectively. Chemical shift was reported in ppm down field from internal TMS. Organic solvents used were dried by standard methods when necessary. Commercially available reagents were used without further purification. All reactions were monitored by TLC with Huanghai $\mathrm{GF}_{254}$ silica gel coated plates. Flash column chromatography was carried out using 300-400 mesh silica gel at increased pressure. All reactions were performed under argon using standard Schlenk techniques. Infrared spectra were recorded on a Perkin-Elmer PE-983 spectrometer with absorption in $\mathrm{cm}^{-1}$. Mass spectra were recorded by ESI and HRMS was measured on a HP-5989 instrument. The $15 \mathrm{~W}$ Blue LED (Manufacturer: Liangyuan-Light Factory, Model: PAR 38, Wavelength: $425 \mathrm{~nm}$ ) was directly purchased from the supermarket. 


\section{General Procedure for the Synthesis of Substrates.}
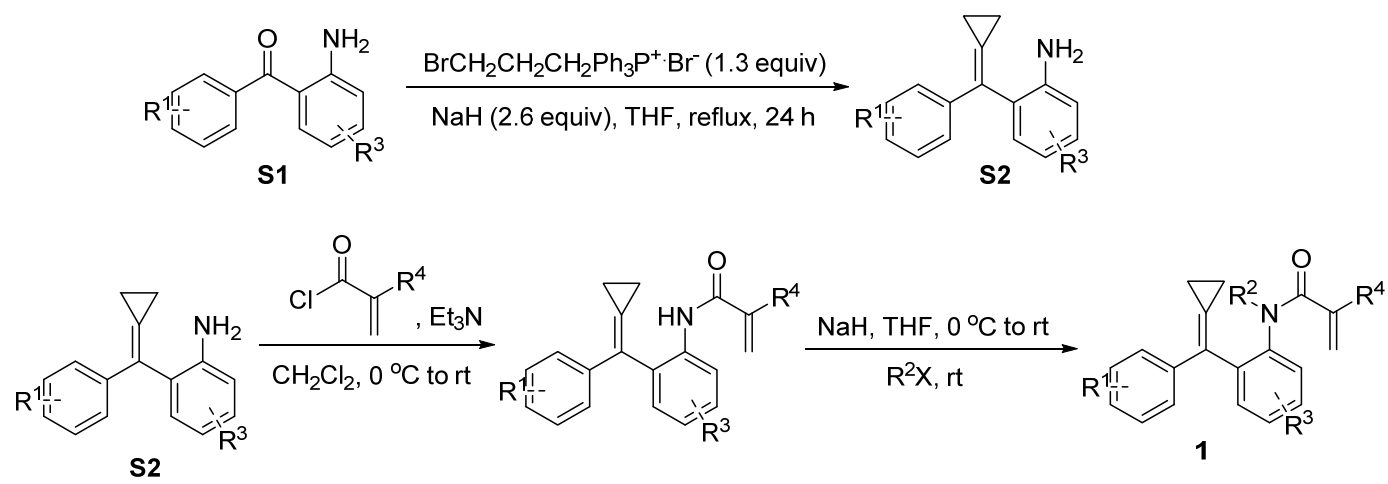

The procedure of preparing substrates $\mathbf{1}$ was slightly modified according to the previous literature. ${ }^{1}$ A solution of 3-bromopropyltriphenylphosphonium bromide (5.96 g, $13 \mathrm{mmol})$ and $\mathrm{NaH}$ (624 mg, $26 \mathrm{mmol})$ in THF $(15 \mathrm{~mL})$ was stirred at $65{ }^{\circ} \mathrm{C}$ in an oil bath under $\mathrm{N}_{2}$ for $12 \mathrm{~h}$. Afterwards compound S1 $(10 \mathrm{mmol})$ in THF $(10 \mathrm{~mL})$ was added and the reaction solution was stirred at $65{ }^{\circ} \mathrm{C}$ in an oil bath for another $12 \mathrm{~h}$. Upon completion, the reaction was cooled to room temperature and the mixture was filtered through a celite. The filtrate was concentrated under reduced pressure and the residue was purified by a silica gel flash chromatography $(\mathrm{PE} / \mathrm{EA}=80 / 1)$ to afford the products $\mathbf{S} 2$ in moderate yields.

A solution of compound S2 $(2.0 \mathrm{mmol})$ in $\mathrm{CH}_{2} \mathrm{Cl}_{2}(5.0 \mathrm{~mL})$ was stirred at $0{ }^{\circ} \mathrm{C}$ in an ice bath under $\mathrm{N}_{2}$. Then $\mathrm{Et}_{3} \mathrm{~N}(3.0 \mathrm{mmol})$ and the corresponding acyl chloride $(2.4 \mathrm{mmol})$ were added dropwise respectively and the resulting mixture was warmed to room temperature and stirred overnight. Upon completion, the mixture was evaporated to remove the solvent and excess methacyloyl. The residue dissolved in THF $(5.0 \mathrm{~mL})$ was added to $\mathrm{NaH}(3.0 \mathrm{mmol})$ in $\mathrm{THF}(5.0 \mathrm{~mL})$ at $0{ }^{\circ} \mathrm{C}$ in an ice bath dropwise and the reaction mixture was stirred for $30 \mathrm{~min}$. Afterwards iodomethane or alkyl bromide (3.0 mmol) was added and the reaction was stirred overnight at room temperature. The reaction mixture was quenched by water and extracted with DCM for 3 times. The combined organic layer was washed with brine and dried over anhydrous $\mathrm{Na}_{2} \mathrm{SO}_{4}$. The solvent was removed under vacuum and the residue was purified by a flash column chromatography on silica gel $(\mathrm{PE} / \mathrm{EA}=10 / 1)$ to afford the products 1 . 


\subsection{Method A:}

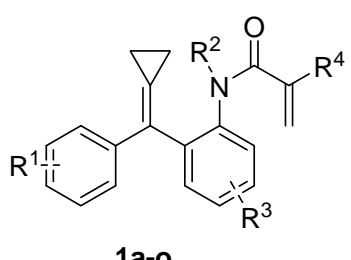

1a-o

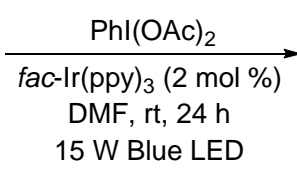

15 W Blue LED

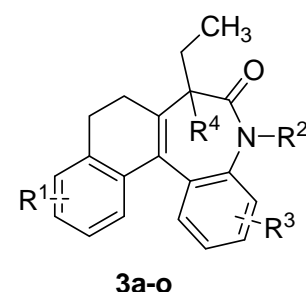

3a-o

An oven-dried Schlenk tube $(20 \mathrm{~mL})$ was equipped with a magnetic stir bar, and substrates 1a-o (0.2 mmol, $60.6 \mathrm{mg})$, fac- $\mathrm{Ir}(\mathrm{ppy})_{3}\left(2.6 \mathrm{mg}, 0.02\right.$ equiv), $\mathrm{PhI}(\mathrm{OAc})_{2}(2.0$ equiv, $64.4 \mathrm{mg})$ and $\mathrm{DMF}$ $(1.0 \mathrm{~mL})$ were added. The tube was degassed by alternating vacuum evacuation (10 min) and argon backfill three times. The tube was placed at a distance (app. $5 \mathrm{~cm}$ ) from $15 \mathrm{~W}$ Blue LED, and the resulting yellow solution was stirred at ambient temperature under visible-light irradiation. When the reaction finished, the mixture was diluted with ethyl acetate and added to a separating funnel containing $15 \mathrm{~mL}$ saturated $\mathrm{K}_{2} \mathrm{CO}_{3}$ aqueous solution. The layers were separated and the aqueous layer was extracted with ethyl acetate $(3 \times 15 \mathrm{~mL})$. The combined organic layers were washed with saturated brine, dried $\left(\mathrm{Na}_{2} \mathrm{SO}_{4}\right)$ and filtered. The filtrate was concentrated in vacuo and the residue was purified by chromatography on a silica gel $(\mathrm{PE} / \mathrm{EA}=10 / 1)$ to afford the products 3a-o.

\subsection{Method B:}<smiles>C=C(C)C(=O)N(C)c1ccccc1C(=C1CC1)c1ccccc1</smiles>

1a

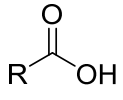

$\mathrm{R}=1^{\circ}, 2^{\circ}, 3^{\circ}$ alkyl

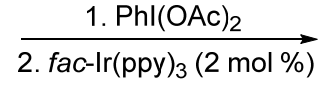
DMF, rt, $24 \mathrm{~h}$ 15 W Blue LED

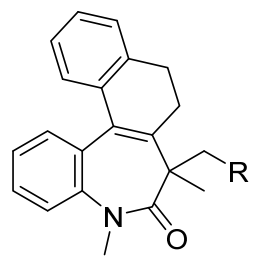

$3 p-3 a d$

In a $25 \mathrm{~mL}$ round flask was equipped with a magnetic stir bar, aliphatic carboxylic acid (5.0 mmol, 2.0 equiv), $\mathrm{PhI}(\mathrm{OAc})_{2}$ (2.5 mmol, 1.0 equiv) and $\mathrm{CHCl}_{3}(15 \mathrm{~mL})$ were added. The phenyliodine(III) dicarboxylate can be easily obtained as a white solid or a viscous oil at $30-40{ }^{\circ} \mathrm{C}$ under reduced pressure to remove the HOAc, ${ }^{2}$ and it can be directly used without further purification. Then, an oven-dried Schlenk tube $(20 \mathrm{~mL})$ was equipped with a magnetic stir bar, 1a $(0.2 \mathrm{mmol}, 60.6 \mathrm{mg})$, fac- $\operatorname{Ir}(\mathrm{ppy})_{3}(2.6 \mathrm{mg}, 0.02$ equiv), phenyliodine(III) dicarboxylates (2.0 equiv, $0.4 \mathrm{mmol})$ and DMF 
$(1.0 \mathrm{~mL})$ were added. The tube was degassed by alternating vacuum evacuation (10 min) and argon backfill three times. The tube was placed at a distance (app. $5 \mathrm{~cm}$ ) from $15 \mathrm{~W}$ Blue LED, and the resulting yellow solution was stirred at ambient temperature under visible-light irradiation. When the reaction finished, the mixture was diluted with ethyl acetate and added to a separating funnel containing $15 \mathrm{~mL}$ saturated $\mathrm{K}_{2} \mathrm{CO}_{3}$ solution. The layers were separated and the aqueous layer was extracted with ethyl acetate $(3 \times 15 \mathrm{~mL})$. The combined organic layers were washed with saturated brine, dried $\left(\mathrm{Na}_{2} \mathrm{SO}_{4}\right)$ and filtered. The filtrate was concentrated in vacuo and the residue was purified by a chromatography $(\mathrm{PE} / \mathrm{EA}=10 / 1)$ on a silica gel to afford the products $\mathbf{3 p}$-3ad.

\subsection{Synthetic Application of the Reaction}

\section{A scale up experiment of 1a.}
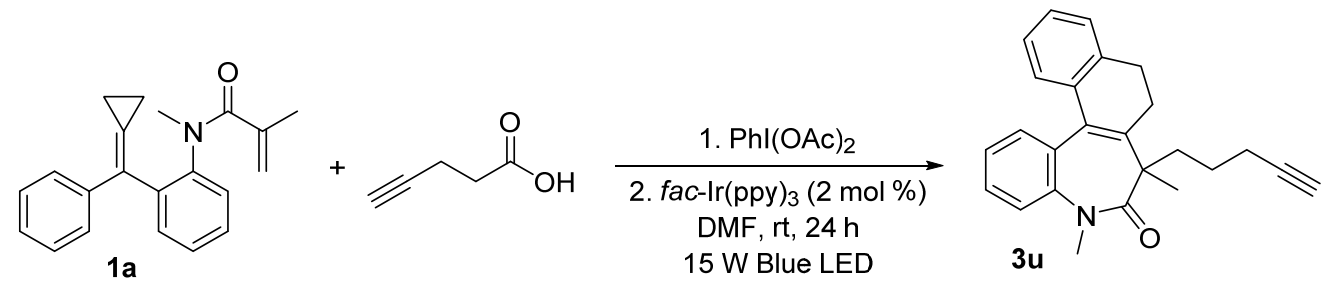

In a $50 \mathrm{~mL}$ round flask was equipped with a magnetic stir bar, aliphatic carboxylic acid (12.0 mmol, 2.0 equiv), $\mathrm{PhI}(\mathrm{OAc})_{2}\left(6.0 \mathrm{mmol}, 1.0\right.$ equiv) and $\mathrm{CHCl}_{3}(20 \mathrm{~mL})$ were added. The phenyliodine(III) dicarboxylate can be easily obtained as a white solid or a viscous oil at $30-40{ }^{\circ} \mathrm{C}$ under reduced pressure to remove the HOAc, ${ }^{2}$ and it can be directly used without further purification. Then, an oven-dried Schlenk tube $(20 \mathrm{~mL})$ was equipped with a magnetic stir bar, 1a (2.0 mmol, $0.61 \mathrm{~g})$, fac- $\operatorname{Ir}(\mathrm{ppy})_{3}(26 \mathrm{mg}, 0.02$ equiv), phenyliodine(III) dicarboxylate (2.0 equiv, $4.0 \mathrm{mmol})$ and DMF $(10 \mathrm{~mL})$ were added. The tube was degassed by alternating vacuum evacuation (10 min) and argon backfill three times. The tube was placed at a distance (app. $5 \mathrm{~cm}$ ) from $15 \mathrm{~W}$ Blue LED, and the resulting yellow solution was stirred at ambient temperature under visible-light irradiation. When the reaction finished, the mixture was diluted with ethyl acetate and added to a separating funnel containing $30 \mathrm{~mL}$ saturated $\mathrm{K}_{2} \mathrm{CO}_{3}$ solution. The layers were separated and the aqueous layer was extracted with ethyl acetate $(3 \times 15 \mathrm{~mL})$. The combined organic layers were washed with saturated brine, dried $\left(\mathrm{Na}_{2} \mathrm{SO}_{4}\right)$ and filtered. The filtrate was concentrated in vacuo and the residue was purified by a chromatography $(\mathrm{PE} / \mathrm{EA}=10 / 1)$ on silica gel to afford the product $3 \mathbf{u}(70 \%$ yield). 


\section{Synthesis of Compound 4a}
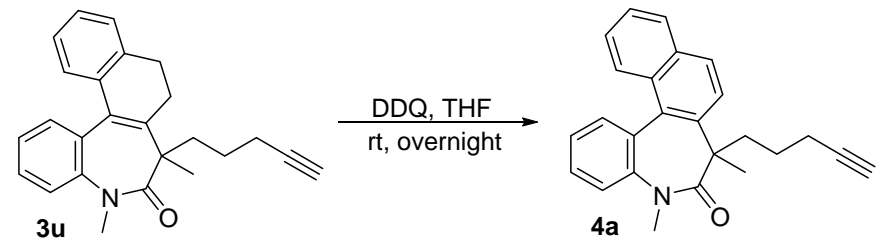

In an oven-dried $20 \mathrm{~mL}$ round flask was equipped with a magnetic stir bar, substrate $3 \mathbf{u}(0.5 \mathrm{mmol}$, 1.0 equiv) and THF ( $5 \mathrm{~mL})$. DDQ ( $0.6 \mathrm{mmol}, 1.2$ equiv) were added in one portion. The resulting mixture was stirred vigorously at room temperature for overnight. After that, the mixture was filtered through a pad of celite and rinsed with EtOAc. The filtrate was concentrated under reduced pressure. The residue was purified by a flash column chromatography $(\mathrm{PE} / \mathrm{EA}=10 / 1)$ on silica gel to afford 4a as foam-like solid (98\% yield).

\section{Synthesis of Compound $4 b$}
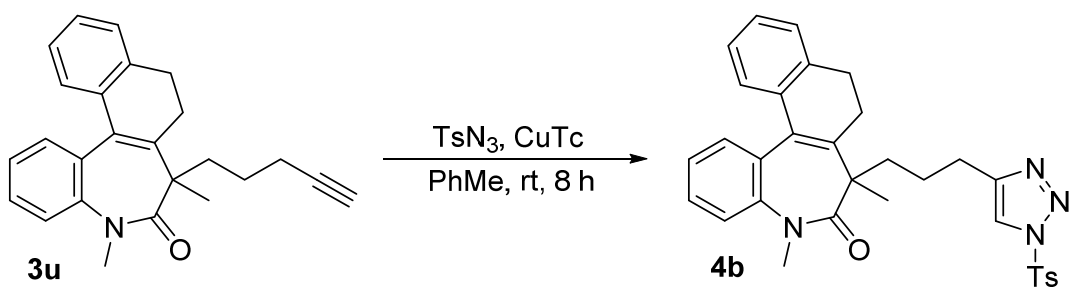

In an oven-dried $10 \mathrm{~mL}$ Schlenk tube was equipped with a magnetic stir bar, substrate $3 \mathbf{u}(0.5 \mathrm{mmol}$, 1.0 equiv), $\mathrm{CuTc}\left(0.05 \mathrm{mmol}, 0.1\right.$ equiv) and $\mathrm{PhMe}(5 \mathrm{~mL})$ were added. After 10 minutes, $\mathrm{TsN}_{3}(0.6$ mmol, 1.2 equiv) was added in one portion. The resulting mixture was stirred vigorously at room temperature for $8 \mathrm{~h}$. After that, the mixture was concentrated under reduced pressure. The residue was purified by a flash column chromatography $(\mathrm{PE} / \mathrm{EA}=10 / 1)$ on silica gel to afford $\mathbf{4 b}$ as foam-like solid (95\% yield).

\section{Synthesis of Compound 4c}
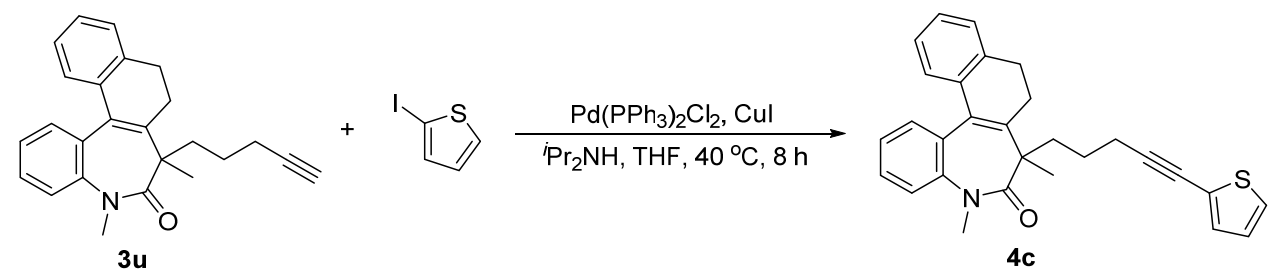

In an oven-dried $20 \mathrm{~mL}$ Schlenk tube was equipped with a magnetic stir bar, $\mathrm{Pd}\left(\mathrm{PPh}_{3}\right)_{2} \mathrm{Cl}_{2}(0.025$ mmol, 0.05 equiv), CuI ( $0.05 \mathrm{mmol}, 0.1$ equiv) were added. The mixture was evacuated and backfilled with argon for three times. Substrate $3 \mathbf{u}(0.5 \mathrm{mmol}, 1.0$ equiv), 2-iodothiophene (0.65 
mmol, 1.3 equiv), ${ }^{i} \operatorname{Pr}_{2} \mathrm{NH}$ (5.0 mmol, 10.0 equiv) and THF ( $\left.8 \mathrm{~mL}\right)$ were added. The Schlenk tube was sealed with a screw-cap and put into a $40{ }^{\circ} \mathrm{C}$ oil bath. After stirring for $8 \mathrm{~h}$, the reaction mixture was cooled to room temperature and concentrated. The residue was purified by a flash column chromatography $(\mathrm{PE} / \mathrm{EA}=10 / 1)$ on silica gel to give $\mathbf{4} \mathbf{c}$ as a colorless oil (98\% yield).

\section{Synthesis of Compound 4d}

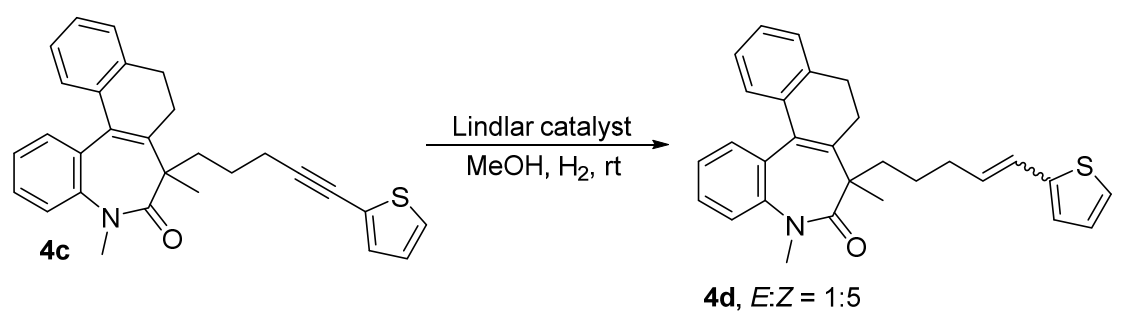

To a flask was added substrate 4c (0.2 mmol, 1.0 equiv), Lindlar catalyst $(20 \mathrm{mg})$ and $\mathrm{MeOH}(8$ $\mathrm{mL}$ ). After the reaction mixture was stirred at room temperature for 24 hours under $1 \mathrm{~atm} \mathrm{H}_{2}$, the resulting mixture was filtered with a pad of celite. The filtrate was concentrated and the residue was purified with a silica gel chromatography $(\mathrm{PE} / \mathrm{EA}=10 / 1)$ to give product $\mathbf{4 d}$ as a colorless oil $(98 \%$ yield). 


\section{Characterization Data of Substrates.}

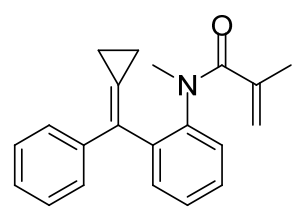

$N$-(2-(cyclopropylidene(phenyl)methyl)phenyl)- $N$-methylmethacrylamide (1a): $462 \mathrm{mg}, 76 \%$ yield, white solid, m.p. $110-112{ }^{\circ} \mathrm{C}$; Eluent: PE/EA = 10/1. ${ }^{1} \mathrm{H}$ NMR (400 $\left.\mathrm{MHz}, \mathrm{CDCl}_{3}, \mathrm{TMS}\right): \delta$ 7.35-7.29 (m, 9H), $4.93(\mathrm{~s}, 1 \mathrm{H}), 4.69(\mathrm{~s}, 1 \mathrm{H}), 2.75(\mathrm{~s}, 3 \mathrm{H}), 1.59(\mathrm{~s}, 3 \mathrm{H}), 1.48-1.39(\mathrm{~m}, 2 \mathrm{H})$, 1.33-1.26 (m, 1H), 1.06-1.00 (m, 1H); $\left.{ }^{13} \mathrm{C} \mathrm{NMR} \mathrm{(100} \mathrm{MHz,} \mathrm{CDCl}_{3}, \mathrm{TMS}\right): \delta 170.2,143.3,140.6$, 139.4, 139.1, 131.9, 128.3, 128.1, 127.8, 127.2, 127.1, 127.0, 119.6, 37.5, 19.8, 4.6, 2.4; IR $\left(\mathrm{CH}_{2} \mathrm{Cl}_{2}\right): v$ 3053, 2973, 1650, 1621, 1594, 1488, 1446, 1362, 767, $697 \mathrm{~cm}^{-1}$; HRMS (ESI) m/z: $(\mathrm{M}+\mathrm{H})^{+}$Calcd. for $\mathrm{C}_{21} \mathrm{H}_{22} \mathrm{NO} 304.1696$; found 304.1696. 


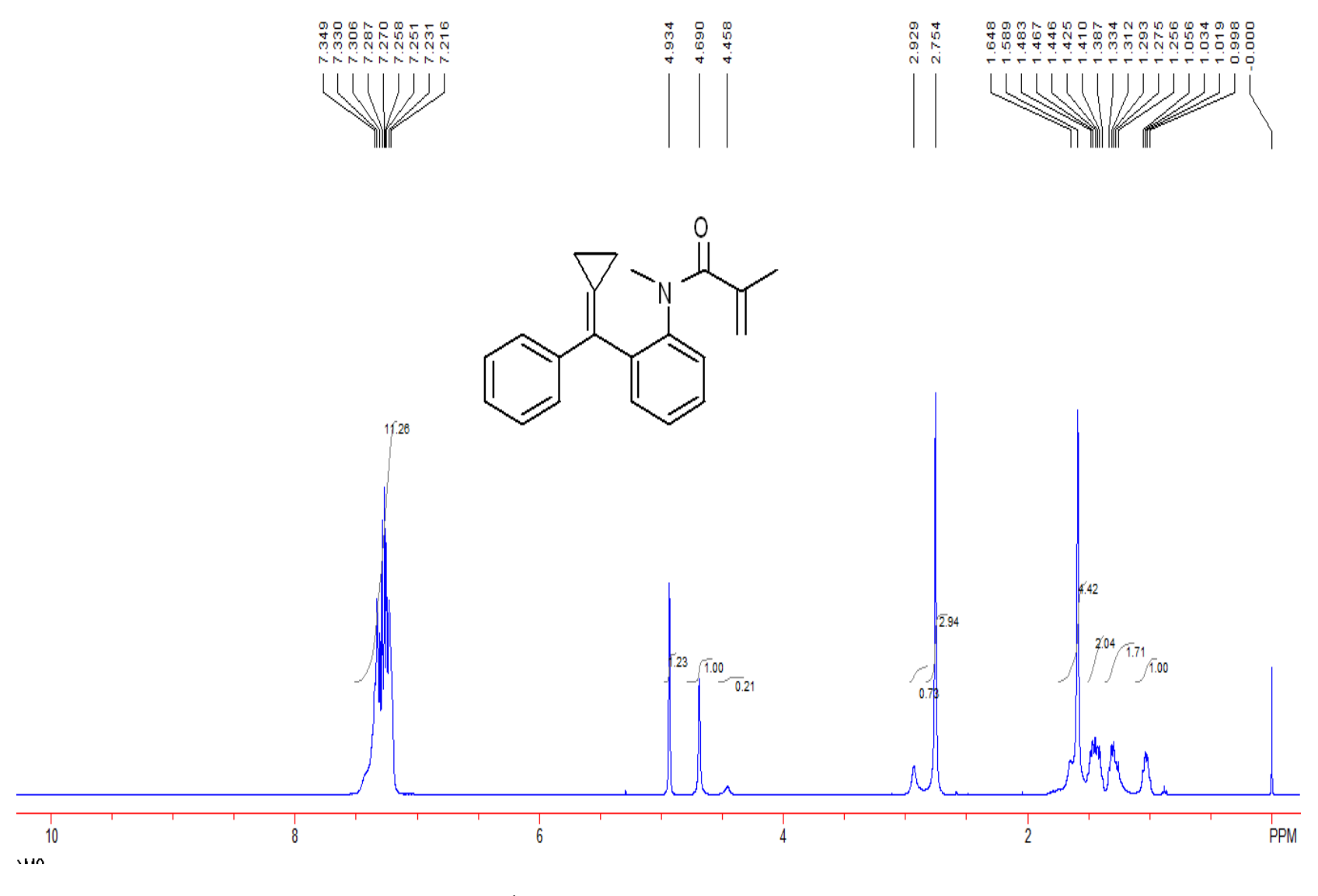

1a $\left({ }^{1} \mathrm{H} \mathrm{NMR}, 400 \mathrm{MHz}, \mathrm{CDCl}_{3}\right)$

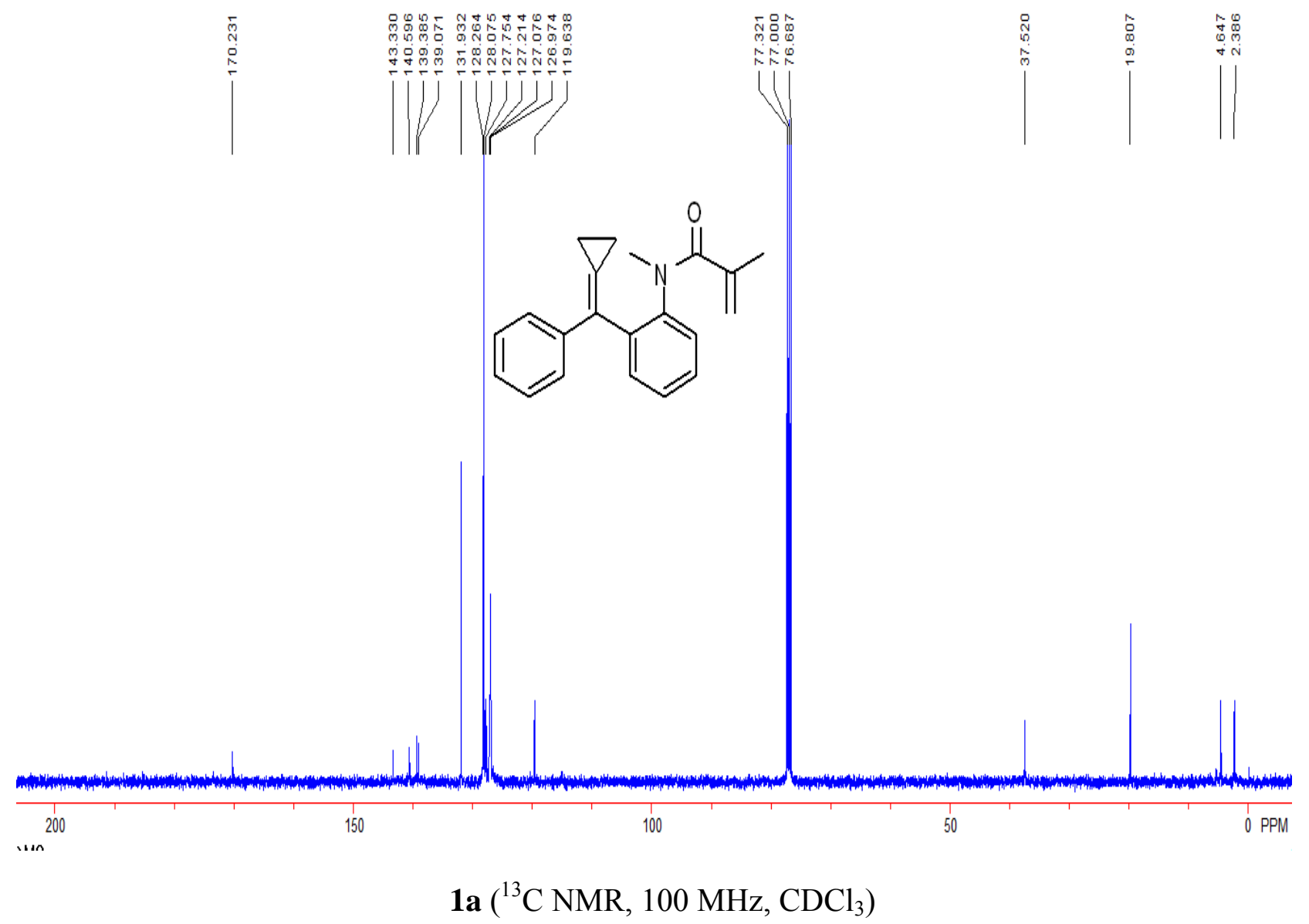




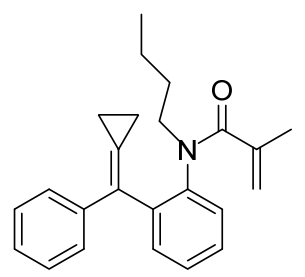

$N$-butyl- $N$-(2-(cyclopropylidene(phenyl)methyl)phenyl)methacrylamide (1b): $478 \mathrm{mg}, 73 \%$ yield, white solid, m.p. $113-115{ }^{\circ} \mathrm{C}$; Eluent: PE/EA = 10/1. ${ }^{1} \mathrm{H}$ NMR (400 MHz, $\left.\mathrm{CDCl}_{3}, \mathrm{TMS}\right): \delta$ 7.44-7.23 (m, 9H), $4.95(\mathrm{~s}, 1 \mathrm{H}), 4.76(\mathrm{~s}, 1 \mathrm{H}), 3.52-3.42(\mathrm{~m}, 1 \mathrm{H}), 2.60-2.57(\mathrm{~m}, 1 \mathrm{H}), 1.80-1.60$ (m, 5H), 1.44-1.42 (m, 2H), 1.30-1.06 (m, 4H), 0.81-0.79 (m, 3H); ${ }^{13} \mathrm{C} \mathrm{NMR}\left(100 \mathrm{MHz}, \mathrm{CDCl}_{3}, \mathrm{TMS}\right)$ : $\delta 170.1,142.8,140.7,140.0,138.6,138.2,132.1,128.5,128.3,128.1,127.9,127.1,126.4,119.8$, 49.8, 30.2, 20.3, 20.0, 13.7, 4.6, 2.7; IR $\left(\mathrm{CH}_{2} \mathrm{Cl}_{2}\right): \vee 2972,2930,1647,1619,1593,1489,1450$, 1367, 771, $698 \mathrm{~cm}^{-1}$; HRMS (ESI) m/z: $(\mathrm{M}+\mathrm{H})^{+}$Calcd. for $\mathrm{C}_{24} \mathrm{H}_{28} \mathrm{NO} 346.2165$; found 346.2172. 


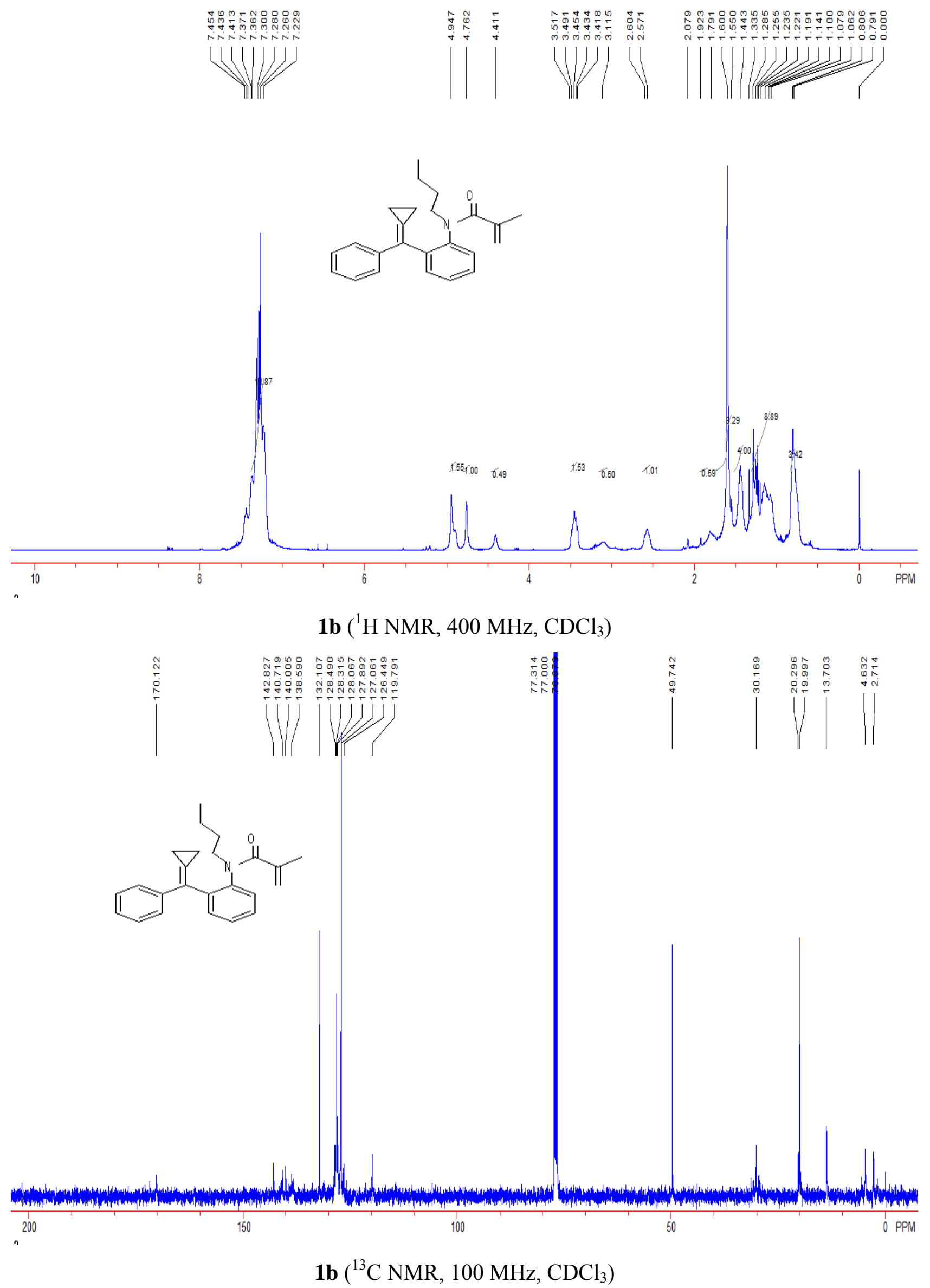




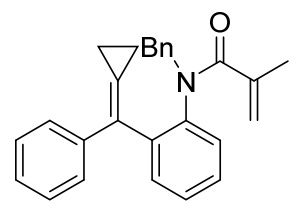

$N$-benzyl- $N$-(2-(cyclopropylidene(phenyl)methyl)phenyl)methacrylamide (1c): $547 \mathrm{mg}, 72 \%$ yield, white solid, m.p. 90-92 ${ }^{\circ} \mathrm{C}$; Eluent: PE/EA = 10/1. ${ }^{1} \mathrm{H}$ NMR (400 $\left.\mathrm{MHz}, \mathrm{CDCl}_{3}, \mathrm{TMS}\right): \delta$ 7.51-6.92 (m, 14H), $4.99(\mathrm{~s}, 1 \mathrm{H}), 4.93(\mathrm{~d}, 1 \mathrm{H}, J=15.2 \mathrm{~Hz}), 4.81(\mathrm{~s}, 1 \mathrm{H}), 3.48(\mathrm{~d}, 1 \mathrm{H}, J=15.2 \mathrm{~Hz})$, $1.62(\mathrm{~s}, 3 \mathrm{H}), 1.50-1.42(\mathrm{~m}, 2 \mathrm{H}), 1.34-1.26(\mathrm{~m}, 1 \mathrm{H}), 1.09-1.07(\mathrm{~m}, 1 \mathrm{H}) ;{ }^{13} \mathrm{C} \mathrm{NMR}\left(100 \mathrm{MHz}, \mathrm{CDCl}_{3}\right.$, TMS): $\delta 170.3,142.7,140.6,139.5,138.7,138.5,131.9,128.3,128.1,127.8,127.4,127.15,127.06$, 126.9, 120.2, 52.5, 19.7, 4.6, 2.5; IR $\left(\mathrm{CH}_{2} \mathrm{Cl}_{2}\right): v$ 3028, 2973, 1651, 1621, 1494, 1487, 1447, 1192, 767, $696 \mathrm{~cm}^{-1}$; HRMS (ESI) m/z: (M+H) ${ }^{+}$Calcd. for $\mathrm{C}_{27} \mathrm{H}_{26} \mathrm{NO} 380.2009$; found 380.2014. 


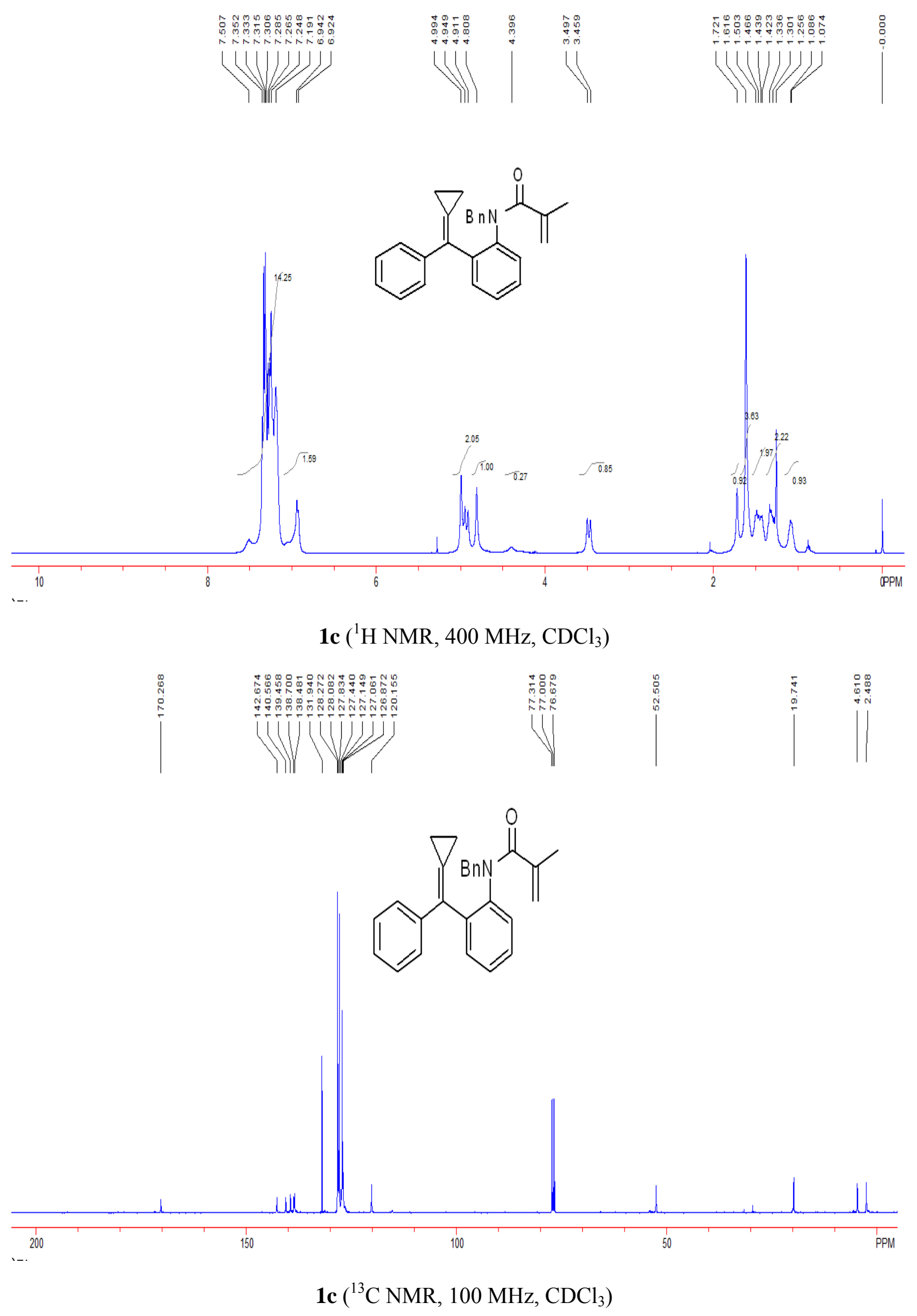




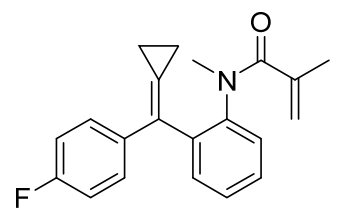

$N$-(2-(cyclopropylidene(4-fluorophenyl)methyl)phenyl)- $N$-methylmethacrylamide (1d): $457 \mathrm{mg}$, 71\% yield, white solid, m.p. 98-100 ${ }^{\circ} \mathrm{C}$; Eluent: PE/EA = 10/1. ${ }^{1} \mathrm{H}$ NMR (400 MHz, $\left.\mathrm{CDCl}_{3}, \mathrm{TMS}\right)$ : $\delta$ 7.36-7.22 (m, 6H), 7.00-6.95 (m, 2H), $4.94(\mathrm{~s}, 1 \mathrm{H}), 4.69(\mathrm{~s}, 1 \mathrm{H}), 2.79(\mathrm{~s}, 3 \mathrm{H}), 1.57(\mathrm{~s}, 3 \mathrm{H})$, 1.46-1.39 (m, 2H), 1.34-1.29 (m, 1H), 1.03-1.02 (m, 1H); $\left.{ }^{13} \mathrm{C} \mathrm{NMR} \mathrm{(100} \mathrm{MHz,} \mathrm{CDCl}_{3}, \mathrm{TMS}\right): \delta$ 170.0, $161.6(\mathrm{~d}, J=245.1 \mathrm{~Hz}), 143.0,139.0,138.5,136.5,131.6,128.4(\mathrm{~d}, J=7.5 \mathrm{~Hz}), 128.3$, 127.5, $127.1(\mathrm{~d}, J=3.0 \mathrm{~Hz}), 127.0,126.9,119.6,114.7(\mathrm{~d}, \mathrm{~J}=21.1 \mathrm{~Hz}), 37.4,19.5,4.4,2.2 ;{ }^{19} \mathrm{~F}$ NMR (376 MHz, $\left.\mathrm{CDCl}_{3}, \mathrm{CFCl}_{3}\right): \delta$-115.0; IR $\left(\mathrm{CH}_{2} \mathrm{Cl}_{2}\right): 3052,2973,1650,1621,1595,1506,1363$, 1222, 1097, $805 \mathrm{~cm}^{-1}$; HRMS (ESI) m/z: $(\mathrm{M}+\mathrm{H})^{+}$Calcd. for $\mathrm{C}_{21} \mathrm{H}_{21} \mathrm{FNO} 322.1602$; found 322.1609 . 


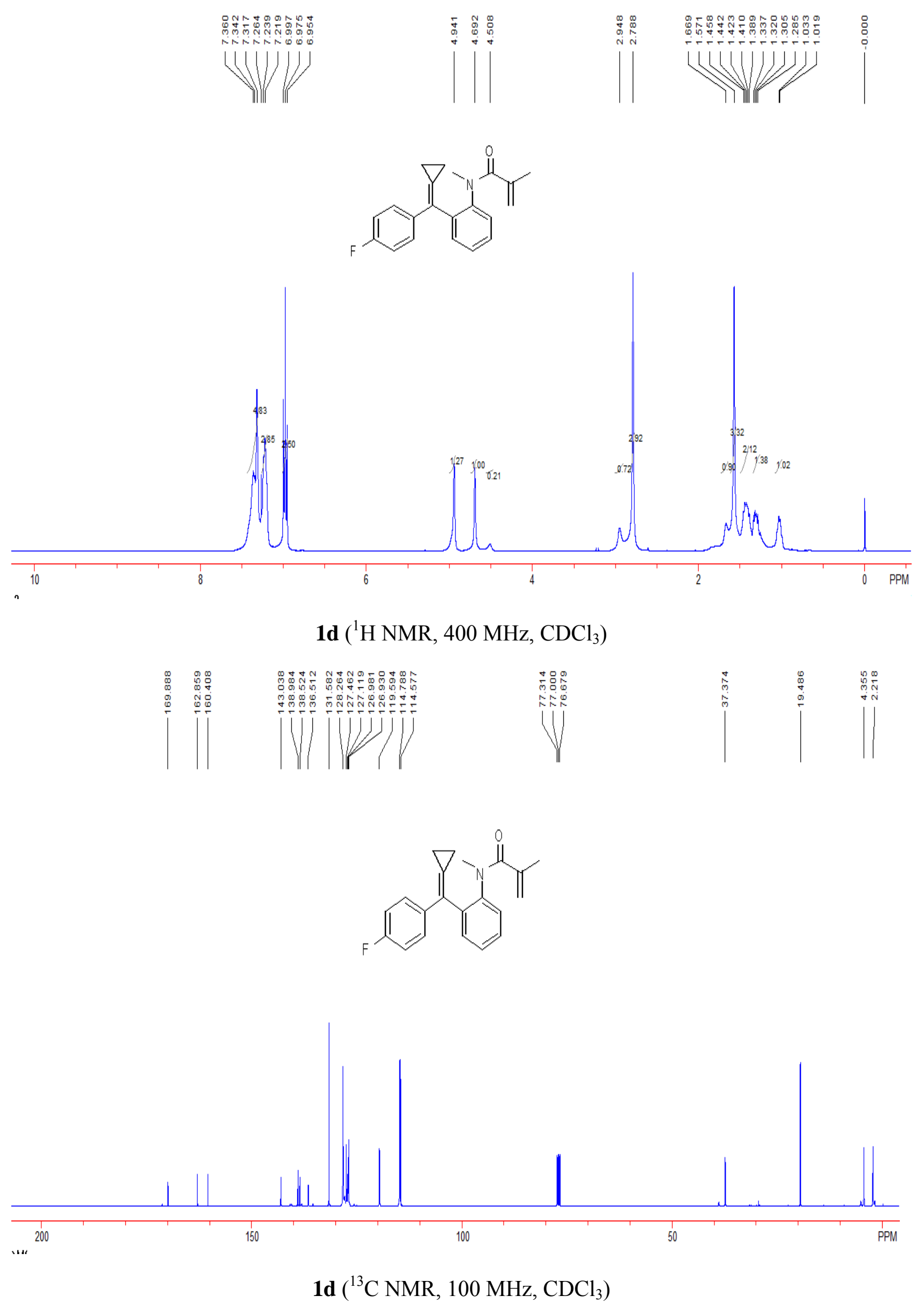




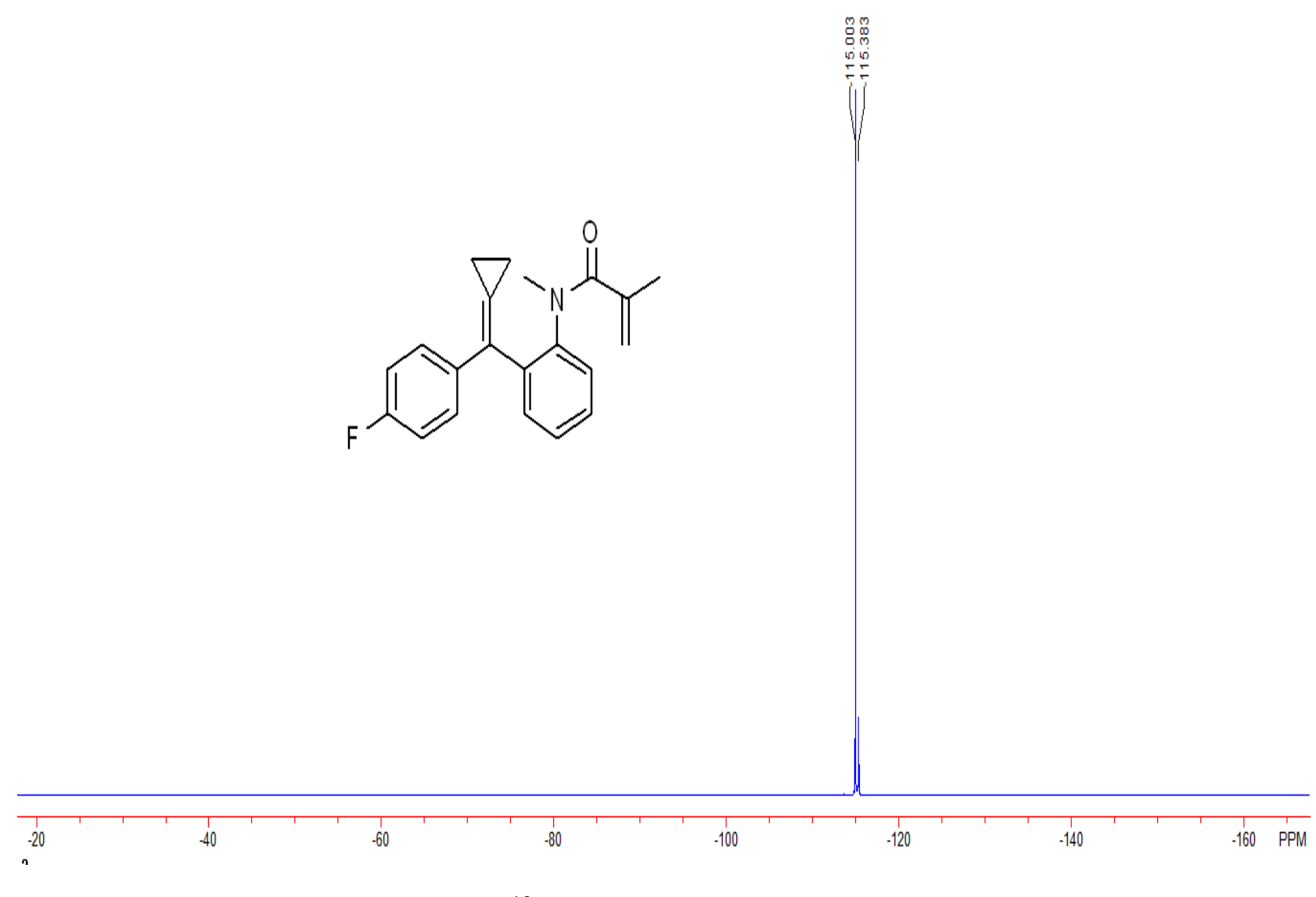

1d $\left({ }^{19} \mathrm{~F}\right.$ NMR, $\left.376 \mathrm{MHz}, \mathrm{CDCl}_{3}\right)$ 


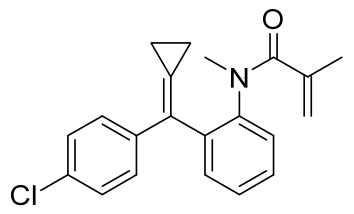

$N$-(2-((4-chlorophenyl)(cyclopropylidene)methyl)phenyl)- $N$-methylmethacrylamide (1e): 487 mg, 72\% yield, white solid, m.p. 103-105 ${ }^{\circ} \mathrm{C}$; Eluent: PE/EA = 10/1. ${ }^{1} \mathrm{H} \mathrm{NMR} \mathrm{(400} \mathrm{MHz,} \mathrm{CDCl}_{3}$, TMS): $\delta$ 7.35-7.22 (m, 6H), 6.99-6.95 (m, 2H), $4.94(\mathrm{~s}, 1 \mathrm{H}), 4.69(\mathrm{~s}, 1 \mathrm{H}), 2.79(\mathrm{~s}, 3 \mathrm{H}), 1.57(\mathrm{~s}, 3 \mathrm{H})$, 1.44-1.39 (m, 2H), 1.34-1.26 (m, 1H), 1.03-1.02 (m, 1H); $\left.{ }^{13} \mathrm{C} \mathrm{NMR} \mathrm{(100} \mathrm{MHz,} \mathrm{CDCl}_{3}, \mathrm{TMS}\right): \delta$ $170.1,163.0,160.6,143.2,139.2,138.7,136.7,131.7,128.45,128.39,127.6,127.3,127.1,119.7$, 114.9, 114.7, 37.5, 19.7, 4.5, 2.4; IR $\left(\mathrm{CH}_{2} \mathrm{Cl}_{2}\right): v$ 3051, 2974, 1649, 1621, 1489, 1361, 1092, 769, $757 \mathrm{~cm}^{-1}$; HRMS (ESI) m/z: $(\mathrm{M}+\mathrm{H})^{+}$Calcd. for $\mathrm{C}_{21} \mathrm{H}_{21} \mathrm{CINO} 338.1306$; found 338.1311. 


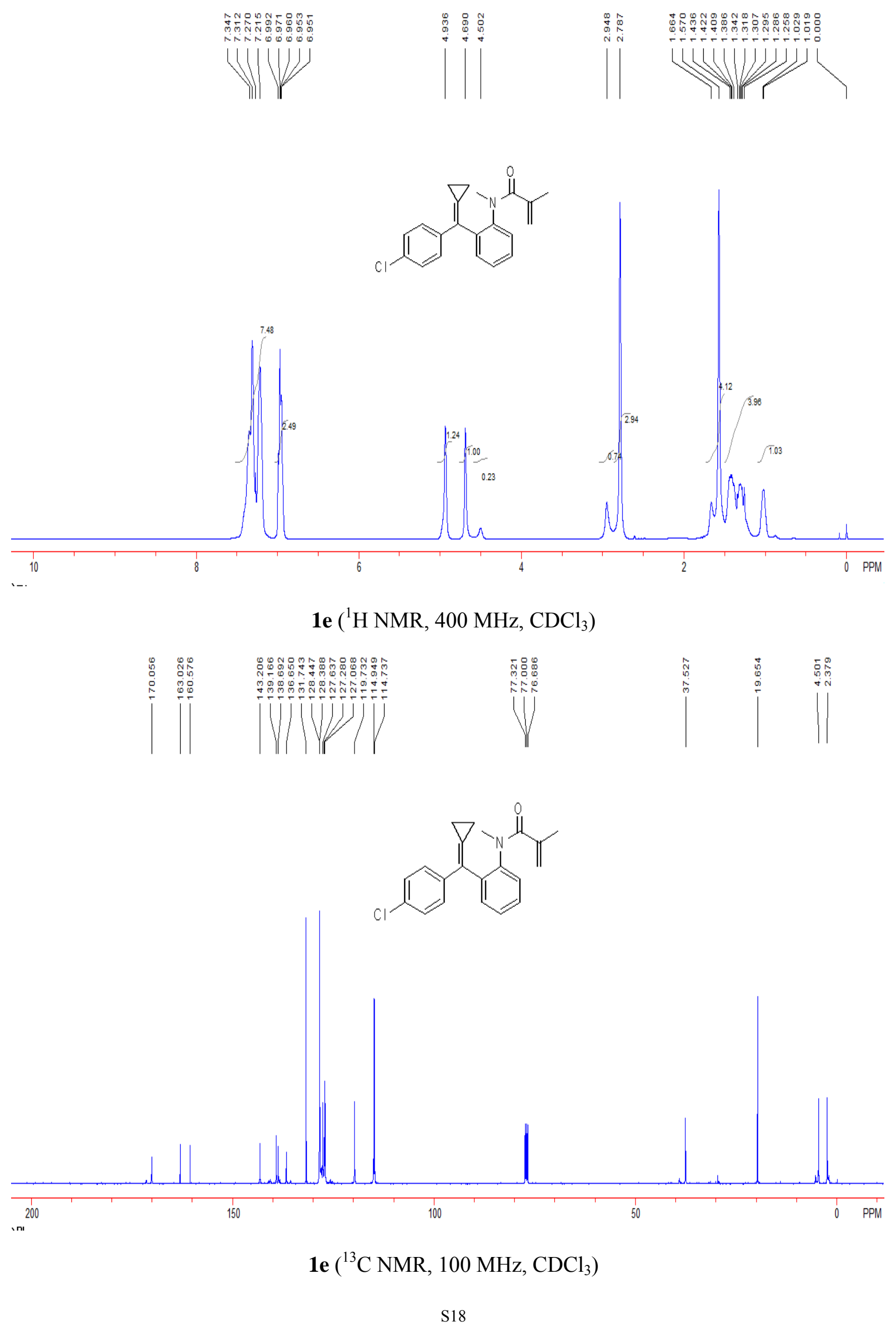




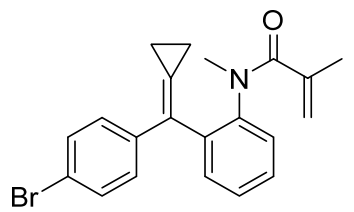

$N$-(2-((4-bromophenyl)(cyclopropylidene)methyl)phenyl)- $N$-methylmethacrylamide (1f): 665 mg, 87\% yield, white solid, m.p. $110-112{ }^{\circ} \mathrm{C}$; Eluent: PE/EA = 10/1. ${ }^{1} \mathrm{H}$ NMR $\left(400 \mathrm{MHz}, \mathrm{CDCl}_{3}\right.$, TMS): $\delta 7.41-7.31(\mathrm{~m}, 6 \mathrm{H}), 7.24(\mathrm{~d}, 1 \mathrm{H}, J=7.6 \mathrm{~Hz}), 7.12(\mathrm{~d}, 1 \mathrm{H}, J=8.0 \mathrm{~Hz}), 4.94(\mathrm{~s}, 1 \mathrm{H}), 4.69$ (s, $1 \mathrm{H}), 2.81(\mathrm{~s}, 3 \mathrm{H}), 1.56(\mathrm{~s}, 3 \mathrm{H}), 1.46-1.39(\mathrm{~m}, 2 \mathrm{H}), 1.34-1.26(\mathrm{~m}, 1 \mathrm{H}), 1.03-1.02(\mathrm{~m}, 1 \mathrm{H}) ;{ }^{13} \mathrm{C} \mathrm{NMR}$ (100 MHz, $\left.\mathrm{CDCl}_{3}, \mathrm{TMS}\right): \delta 170.1,143.3,139.6,139.2,138.4,131.8,131.2,128.52,128.48,128.1$, 127.7, 127.3, 127.1, 120.9, 119.9, 37.7, 19.7, 4.7, 2.5; IR $\left(\mathrm{CH}_{2} \mathrm{Cl}_{2}\right): v 3051,2974,1648,1620,1595$, 1486, 1361, 1100, 1073, 1008, 903, 768, $756 \mathrm{~cm}^{-1}$; HRMS (ESI) m/z: (M+H) Calcd. for $\mathrm{C}_{21} \mathrm{H}_{21} \mathrm{BrNO} 382.0801$; found 382.0805. 

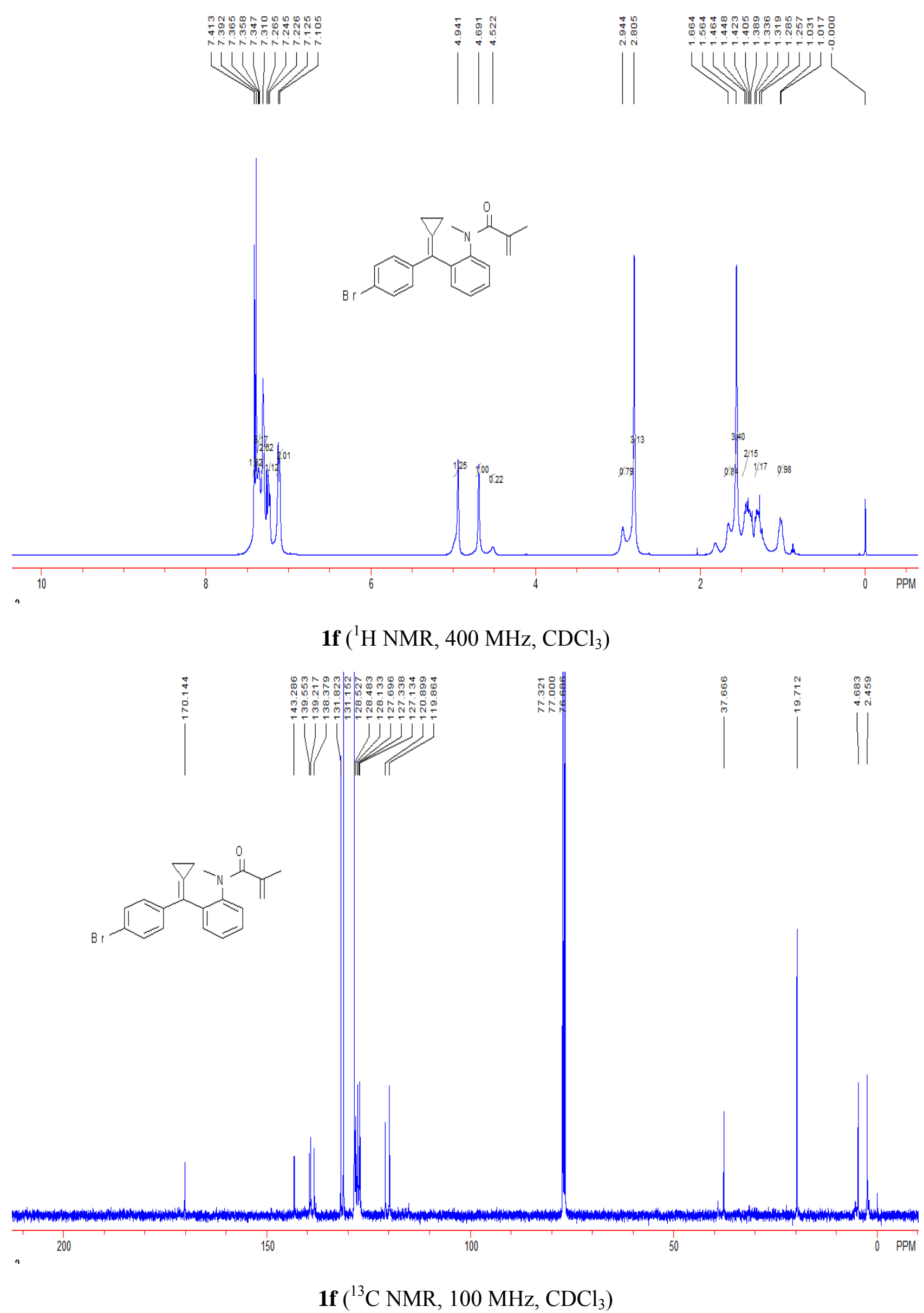


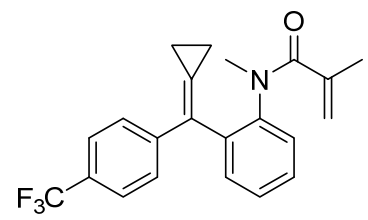

$N$-(2-(cyclopropylidene(4-(trifluoromethyl)phenyl)methyl)phenyl)- $N$-methylmethacrylamide

(1g): 566 mg, 76\% yield, white solid, m.p. $114-116{ }^{\circ} \mathrm{C}$; Eluent: PE/EA = 10/1. ${ }^{1} \mathrm{H}$ NMR (400 MHz, $\left.\mathrm{CDCl}_{3}, \mathrm{TMS}\right): \delta 7.54(\mathrm{~d}, 2 \mathrm{H}, J=7.6 \mathrm{~Hz}), 7.38-7.25(\mathrm{~m}, 6 \mathrm{H}), 4.96(\mathrm{~s}, 1 \mathrm{H}), 4.70(\mathrm{~s}, 1 \mathrm{H}), 2.77(\mathrm{~s}, 3 \mathrm{H})$, $1.58(\mathrm{~s}, 3 \mathrm{H}), 1.52-1.33(\mathrm{~m}, 3 \mathrm{H}), 1.10-1.07(\mathrm{~m}, 1 \mathrm{H}) ;{ }^{13} \mathrm{C} \mathrm{NMR}\left(100 \mathrm{MHz}, \mathrm{CDCl}_{3}, \mathrm{TMS}\right): \delta 170.1$, $144.2,143.3,139.2,138.1,131.8,129.9,128.9$ (q, $\left.J_{\mathrm{C}-\mathrm{F}}=32.8 \mathrm{~Hz}\right), 128.7,127.7,127.2,127.1$ (q, $\left.J_{\mathrm{C}-\mathrm{F}}=1.0 \mathrm{~Hz}\right), 127.0,125.0\left(\mathrm{q}, J_{\mathrm{C}-\mathrm{F}}=3.7 \mathrm{~Hz}\right), 124.1\left(\mathrm{q}, J_{\mathrm{C}-\mathrm{F}}=270.5 \mathrm{~Hz}\right), 120.0,37.5,19.7,4.7,2.5$; $\left.{ }^{19} \mathrm{~F} \mathrm{NMR} \mathrm{(376} \mathrm{MHz,} \mathrm{CDCl}_{3}, \mathrm{CFCl}_{3}\right): \delta-62.3$; IR $\left(\mathrm{CH}_{2} \mathrm{Cl}_{2}\right): v$ 2976, 1650, 1620, 1323, 1163, 1115 , 1069, 848, $768 \mathrm{~cm}^{-1}$; HRMS (ESI) m/z: (M+H) Calcd. for $\mathrm{C}_{22} \mathrm{H}_{21} \mathrm{~F}_{3} \mathrm{NO} 372.1570$; found 372.1584 . 


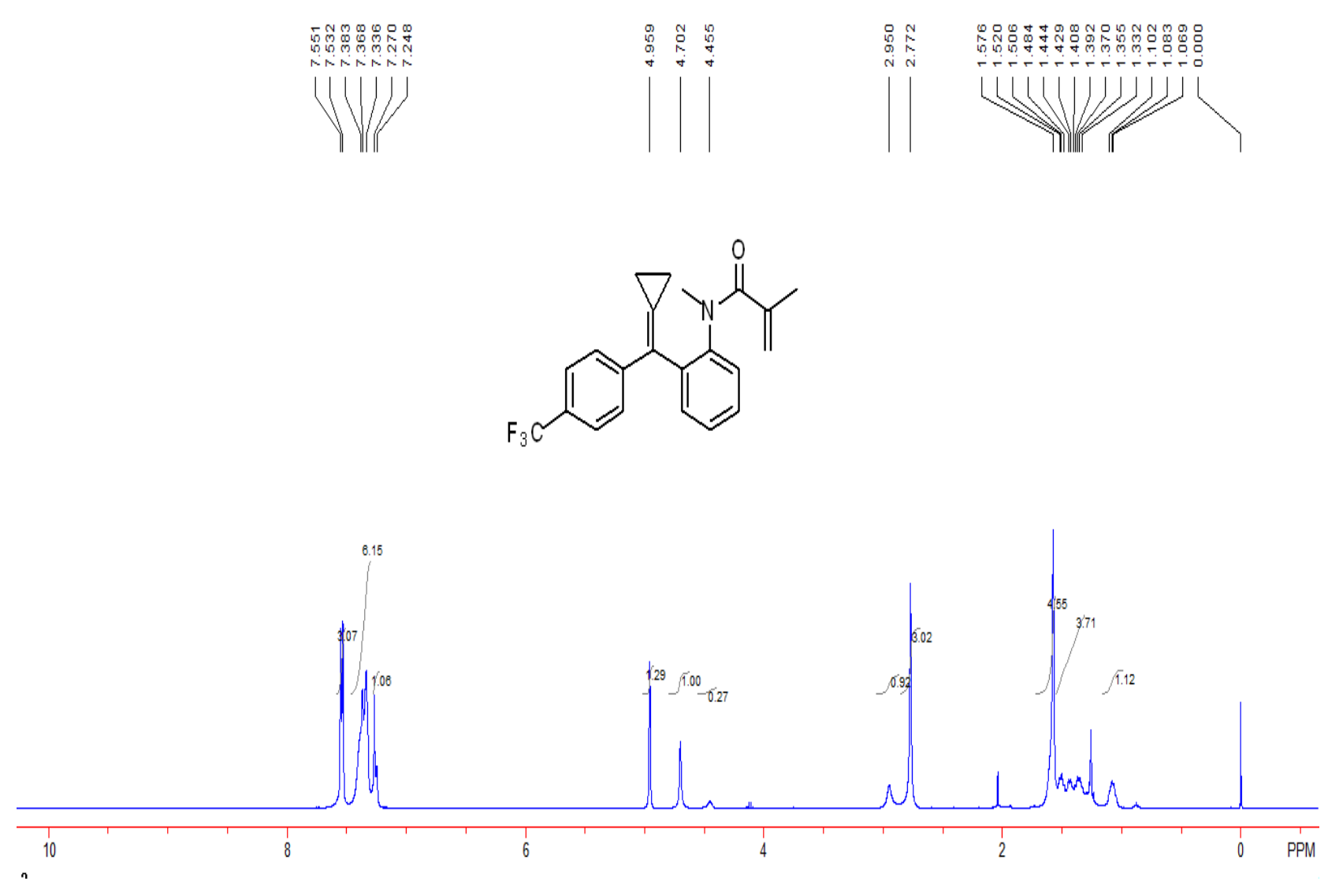

1 g $\left({ }^{1} \mathrm{H} \mathrm{NMR}, 400 \mathrm{MHz}, \mathrm{CDCl}_{3}\right)$

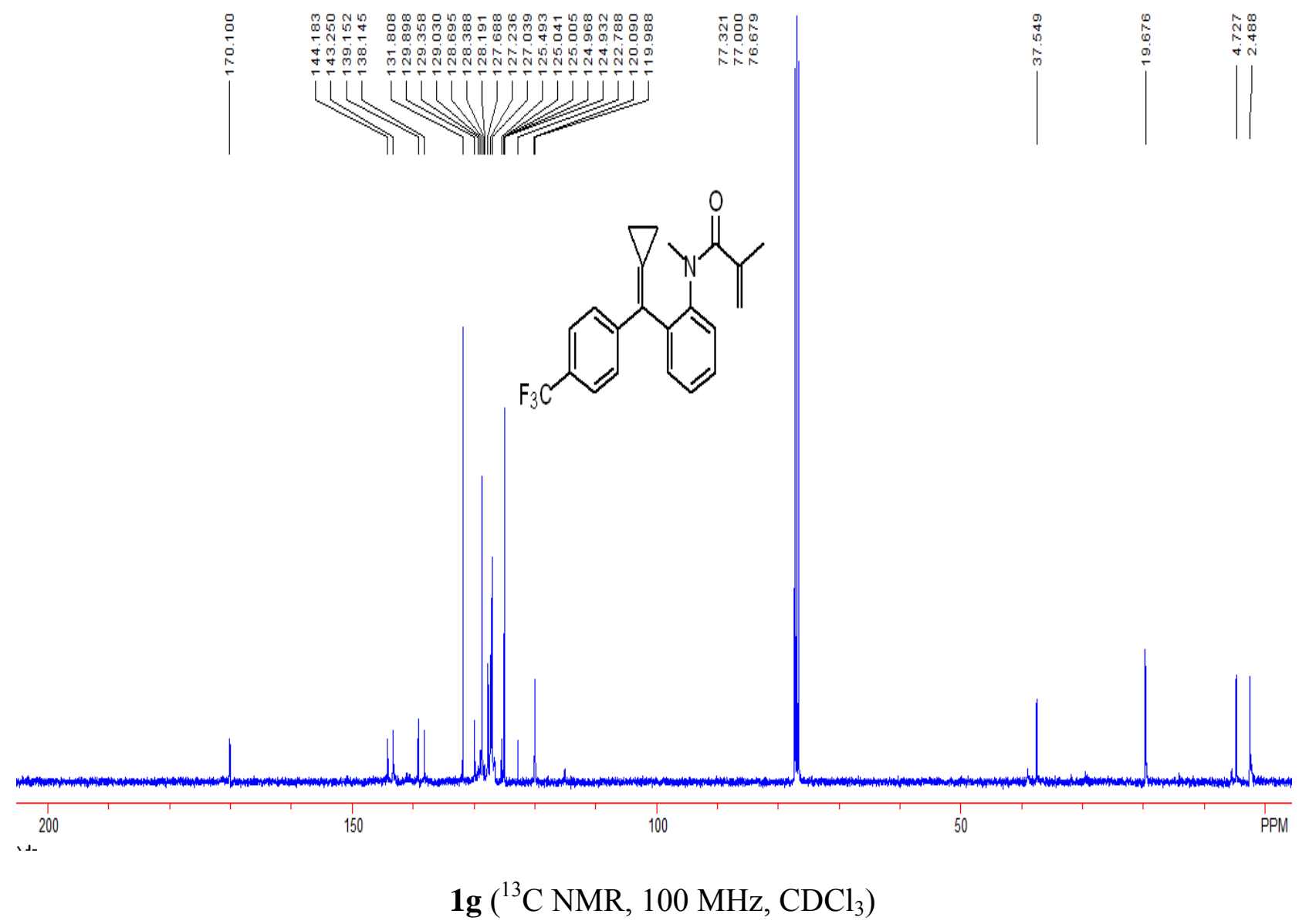




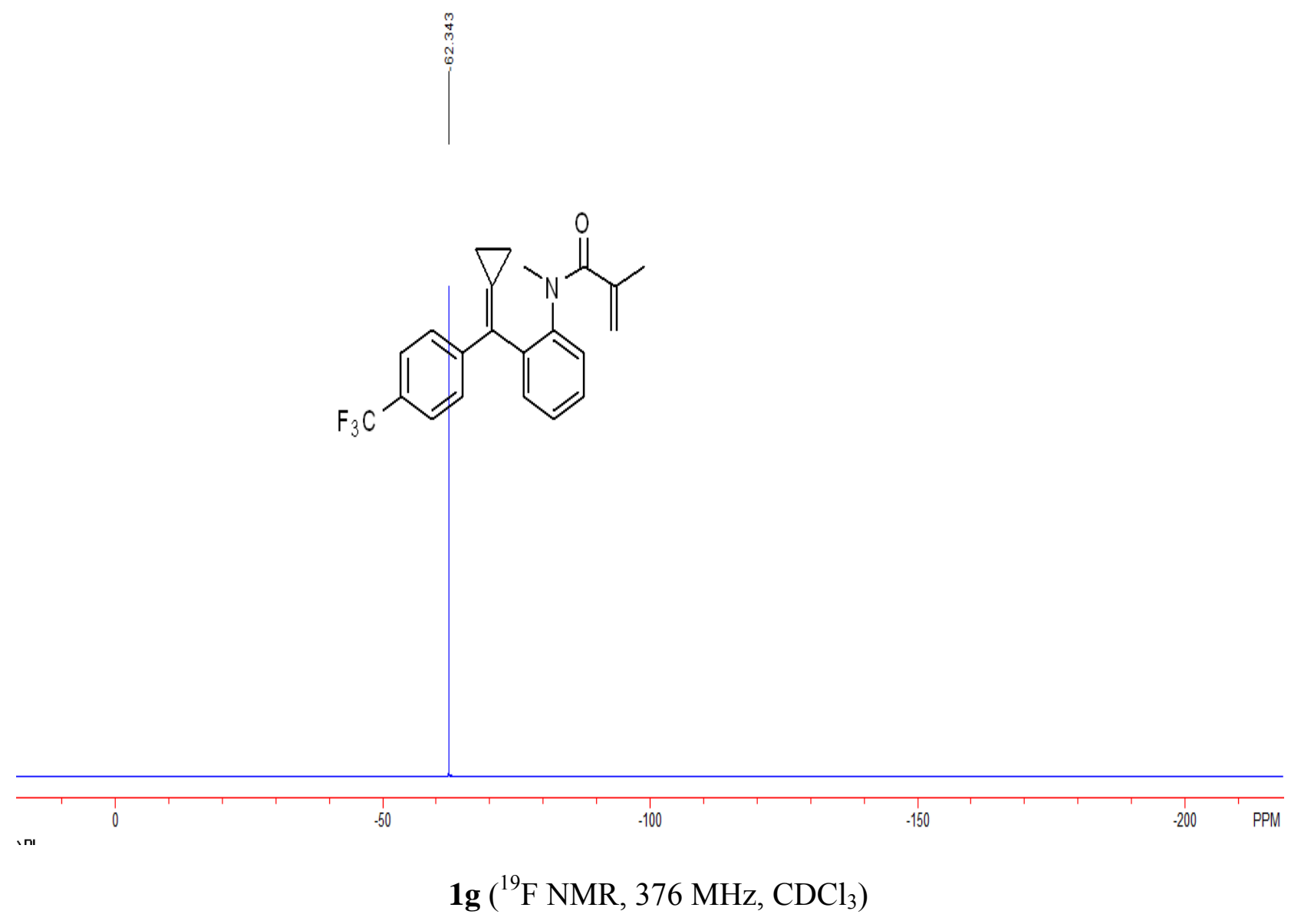




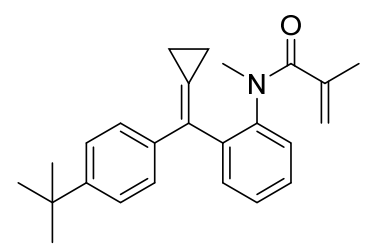

$N$-(2-((4-(tert-butyl)phenyl)(cyclopropylidene)methyl)phenyl)- $N$-methylmethacrylamide (1h):

$548 \mathrm{mg}, 76 \%$ yield, colorless liquid; Eluent: PE/EA = 10/1. ${ }^{1} \mathrm{H} \mathrm{NMR}$ (400 $\left.\mathrm{MHz}, \mathrm{CDCl}_{3}, \mathrm{TMS}\right): \delta$ 7.33-7.29 (m, 6H), 7.19 (t, 2H, J=8.4 Hz), 4.93 (s, 1H), $4.68(\mathrm{~s}, 1 \mathrm{H}), 2.77$ (s, 3H), 1.59 (s, 3H), 1.47-1.41 (m, 2H), $1.29(\mathrm{~s}, 9 \mathrm{H}), 1.27-1.26(\mathrm{~m}, 1 \mathrm{H}), 1.04-0.99(\mathrm{~m}, 1 \mathrm{H}) ;{ }^{13} \mathrm{C} \mathrm{NMR}\left(100 \mathrm{MHz}, \mathrm{CDCl}_{3}\right.$, TMS): $\delta 170.2,150.0,143.3,139.4,139.1,137.6,131.9,128.1,127.7,127.0,126.6,126.2,124.9$, 119.5, 37.5, 34.4, 31.2, 19.8, 4.6, 2.3; IR $\left(\mathrm{CH}_{2} \mathrm{Cl}_{2}\right): v 2962,2867,1651,1621,1488,1488,1361$, 1105, 907, 837, 768, 758, $734 \mathrm{~cm}^{-1}$; HRMS (ESI) m/z: $(\mathrm{M}+\mathrm{H})^{+}$Calcd. for $\mathrm{C}_{25} \mathrm{H}_{30} \mathrm{NO} 360.2322$; found 360.2322 . 


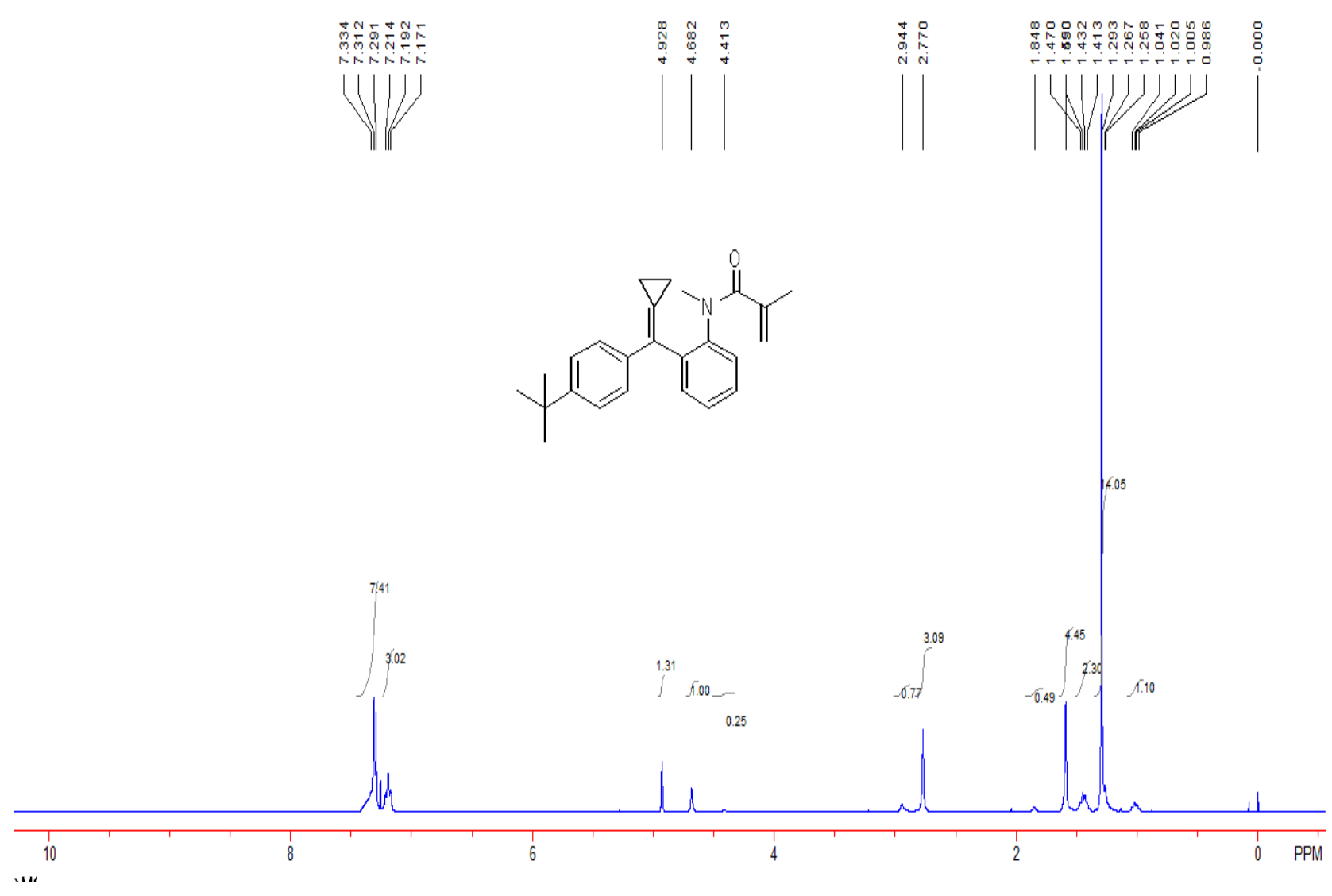

1h $\left({ }^{1} \mathrm{H}\right.$ NMR, $\left.400 \mathrm{MHz}, \mathrm{CDCl}_{3}\right)$

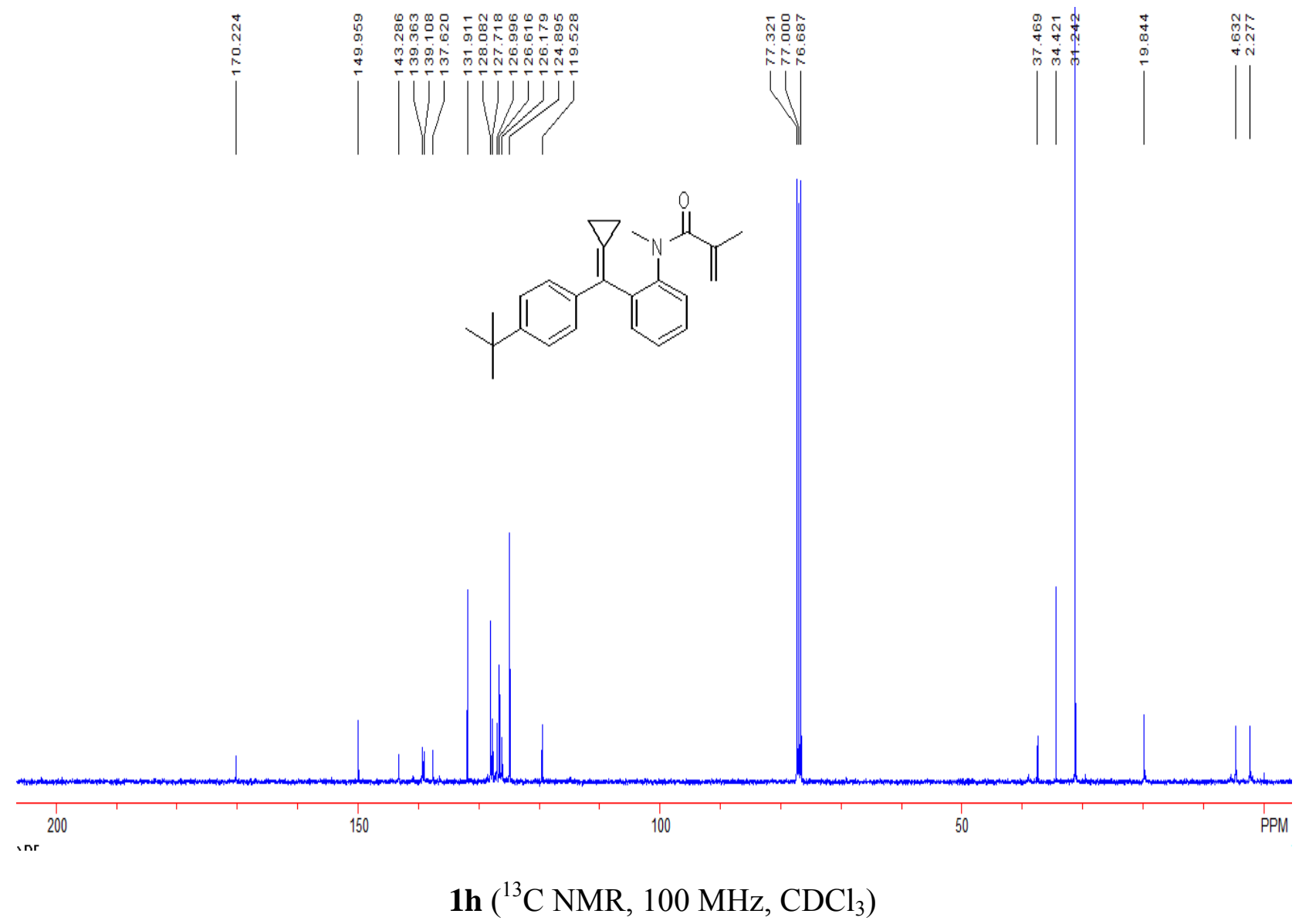




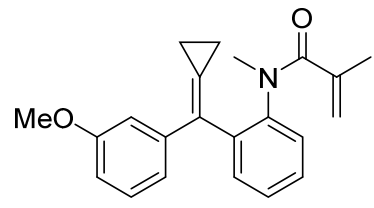

$N$-(2-(cyclopropylidene(3-methoxyphenyl)methyl)phenyl)- $N$-methylmethacrylamide (1i): 515 mg, 77\% yield, white solid, m.p. $120-122{ }^{\circ} \mathrm{C}$; Eluent: PE/EA = 10/1. ${ }^{1} \mathrm{H}$ NMR (400 MHz, $\mathrm{CDCl}_{3}$, TMS): $\delta$ 7.31-7.17 (m, 5H), 6.81-6.77 (m, 3H), $4.93(\mathrm{~s}, 1 \mathrm{H}), 4.69(\mathrm{~s}, 1 \mathrm{H}), 3.73(\mathrm{~s}, 3 \mathrm{H}), 2.81(\mathrm{~s}, 3 \mathrm{H})$, $1.59(\mathrm{~s}, 3 \mathrm{H}), 1.45-1.44(\mathrm{~m}, 2 \mathrm{H}), 1.30-1.28(\mathrm{~m}, 1 \mathrm{H}), 1.03-1.01(\mathrm{~m}, 1 \mathrm{H}) ;{ }^{13} \mathrm{C} \mathrm{NMR}\left(100 \mathrm{MHz}, \mathrm{CDCl}_{3}\right.$, TMS): $\delta 170.1,159.2,143.2,141.9,139.3,138.8,131.8,128.9,128.2,128.1,127.6,127.3,127.0$, 119.6, 119.5, 113.0, 112.0, 55.0, 37.5, 19.7, 4.7, 2.3; IR $\left(\mathrm{CH}_{2} \mathrm{Cl}_{2}\right): v 2972,1650,1621,1596,1578$, 1487, 1449, 1430, 1377, 1362, 1235, 1164, 1043, 768, $697 \mathrm{~cm}^{-1}$; HRMS (ESI) m/z: (M+H) Calcd. for $\mathrm{C}_{22} \mathrm{H}_{24} \mathrm{NO}_{2}$ 334.1802; found 334.1801. 

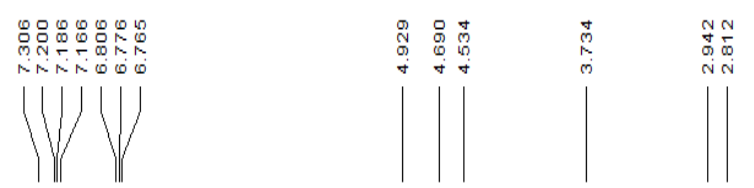

$\mid$<smiles>C=C(C)C(=O)N1C(=C(c2ccccc2)c2cccc(OC)c2)C2CC21</smiles>

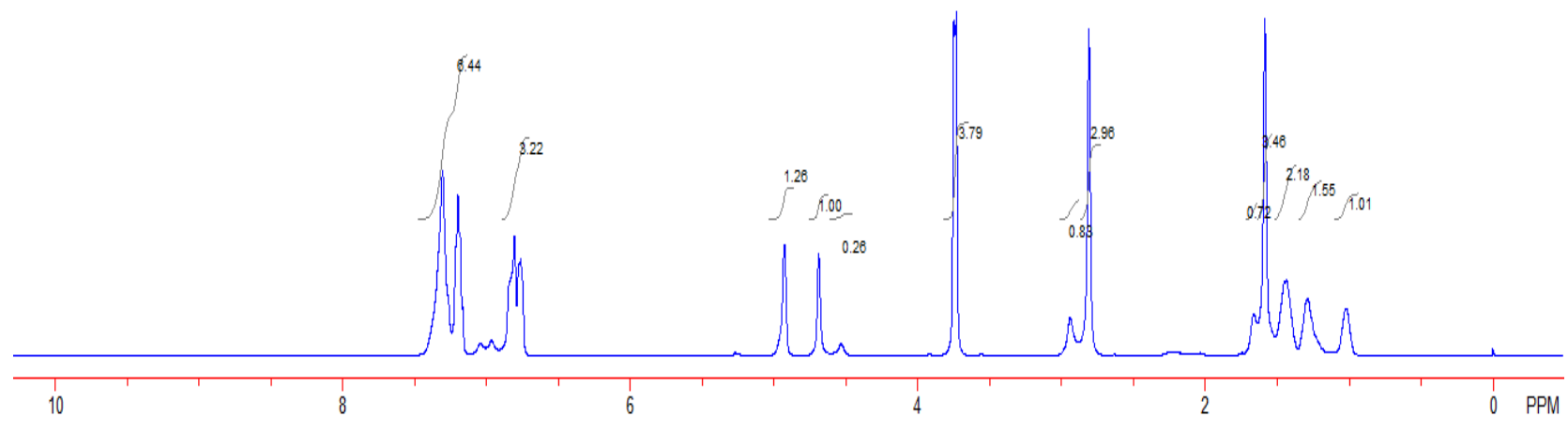

1i ( $\left.{ }^{1} \mathrm{H} \mathrm{NMR}, 400 \mathrm{MHz}, \mathrm{CDCl}_{3}\right)$

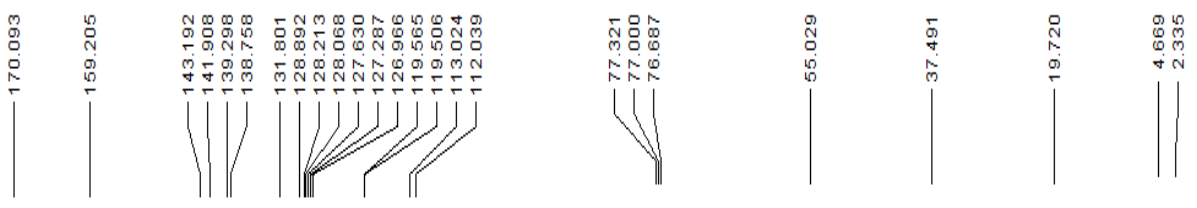<smiles>C=C(C)C(=O)N1c2ccccc2C(c2cccc(OC)c2)=C2CC21</smiles>

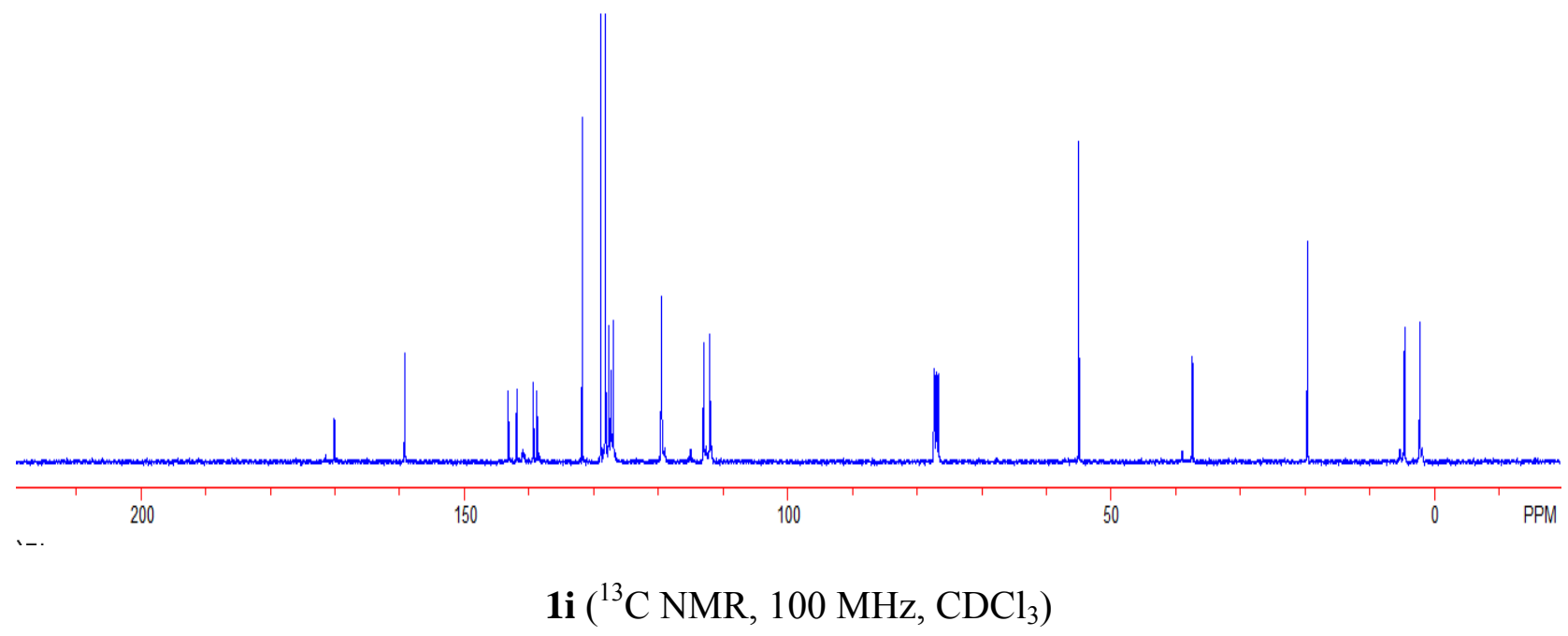




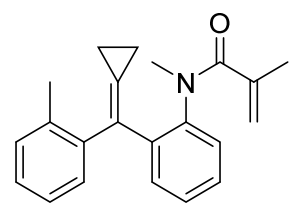

$N$-(2-(cyclopropylidene(o-tolyl)methyl)phenyl)- $N$-methylmethacrylamide (1j): $554 \mathrm{mg}, 87 \%$ yield, white solid, m.p. 97-99 ${ }^{\circ} \mathrm{C}$; Eluent: PE/EA = 10/1. ${ }^{1} \mathrm{H} \mathrm{NMR}\left(400 \mathrm{MHz}, \mathrm{CDCl}_{3}, \mathrm{TMS}\right): \delta 7.45$ $\left(\mathrm{dd}, J_{1}=7.2 \mathrm{~Hz}, J_{2}=1.6 \mathrm{~Hz}, 1 \mathrm{H}\right), 7.33-7.26(\mathrm{~m}, 2 \mathrm{H}), 7.20-7.06(\mathrm{~m}, 5 \mathrm{H}), 4.88(\mathrm{~s}, 1 \mathrm{H}), 4.67(\mathrm{~s}, 1 \mathrm{H})$, $2.48(\mathrm{~s}, 3 \mathrm{H}), 1.98(\mathrm{~s}, 3 \mathrm{H}), 1.63(\mathrm{~s}, 3 \mathrm{H}), 1.47-1.41(\mathrm{~m}, 1 \mathrm{H}), 1.30-1.24(\mathrm{~m}, 2 \mathrm{H}), 1.18-1.12(\mathrm{~m}, 1 \mathrm{H})$; ${ }^{13} \mathrm{C}$ NMR (100 MHz, $\left.\mathrm{CDCl}_{3}, \mathrm{TMS}\right): \delta 170.6,142.2,140.5,140.3,139.9,135.7,131.9,130.3,129.5$, $129.4,128.8,128.4,127.9,127.6,127.2,125.8,118.8,36.3,20.7,20.3,4.1,3.7 ; \mathrm{IR}\left(\mathrm{CH}_{2} \mathrm{Cl}_{2}\right): v$ 3054, 2974, 1649, 1621, 1487, 1449, 1362, 1103, 905, 763, 751, $725 \mathrm{~cm}^{-1}$; HRMS (ESI) m/z: $(\mathrm{M}+\mathrm{H})^{+}$Calcd. for $\mathrm{C}_{22} \mathrm{H}_{24} \mathrm{NO} 318.1852$; found 318.1856 . 


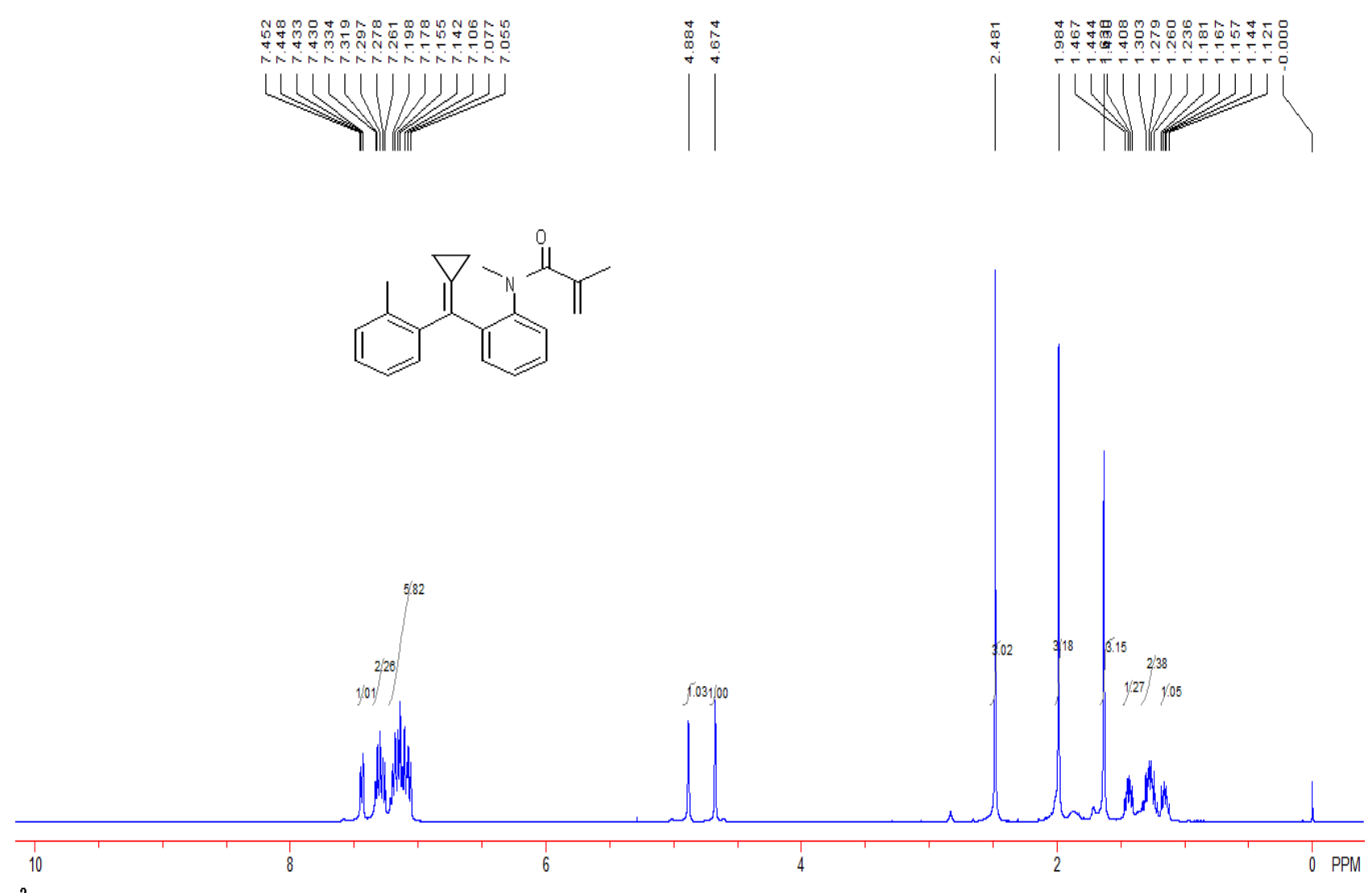

1j $\left({ }^{1} \mathrm{H} \mathrm{NMR}, 400 \mathrm{MHz}, \mathrm{CDCl}_{3}\right)$

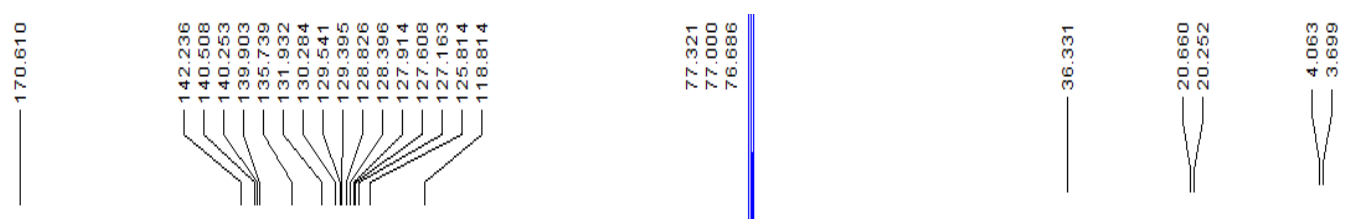<smiles>C=C(C)C(=O)N1c2ccccc2C(c2ccccc2C)=C2CC21</smiles>

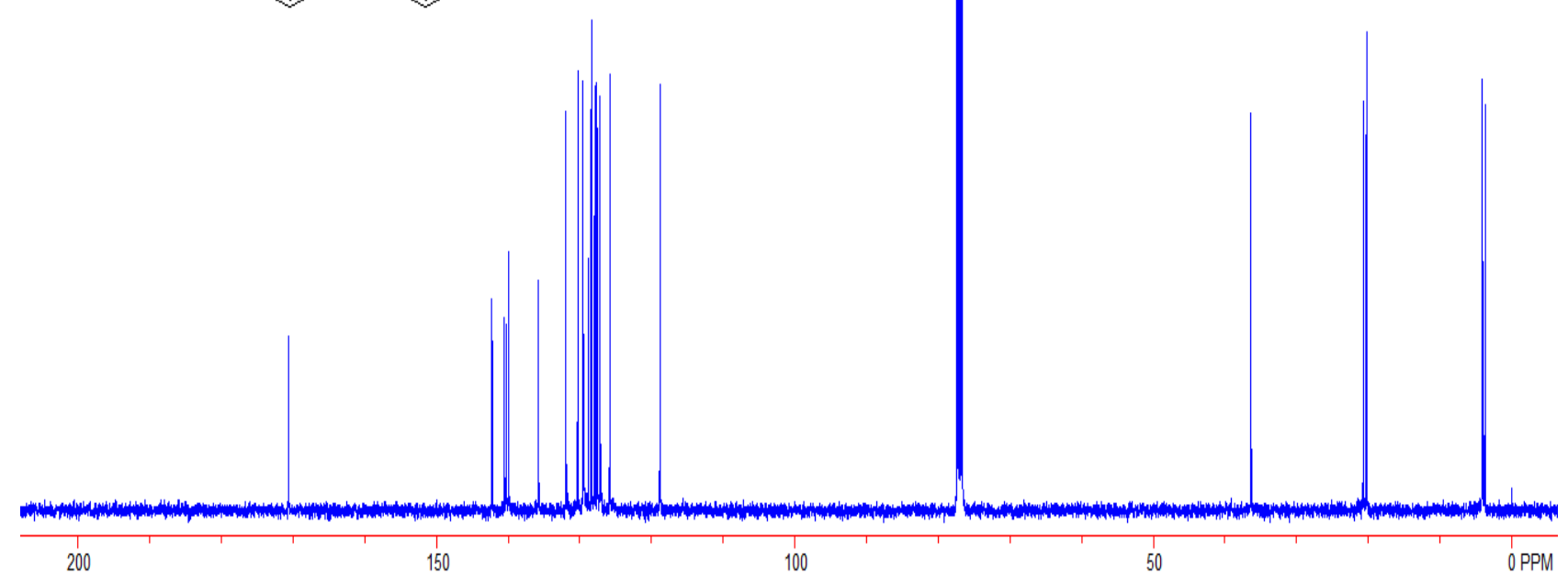

1j $\left({ }^{13} \mathrm{C} \mathrm{NMR,}, 100 \mathrm{MHz}, \mathrm{CDCl}_{3}\right)$ 


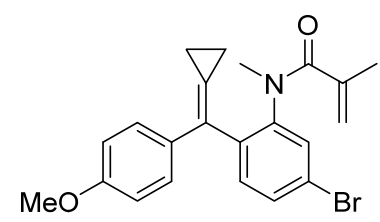

$N$-(5-bromo-2-(cyclopropylidene(4-methoxyphenyl)methyl)phenyl)- $N$-methylmethacrylamide

(1k): 602 mg, 73\% yield, white solid, m.p. 118-120 ${ }^{\circ} \mathrm{C}$; Eluent: PE/EA = 10/1. ${ }^{1} \mathrm{H}$ NMR (400 MHz, $\left.\mathrm{CDCl}_{3}, \mathrm{TMS}\right): \delta 7.41$ (brs, 2H), 7.20-7.18 (m, 3H), 6.83 (d, 2H, J = 8.8 Hz), $4.99(\mathrm{~s}, 1 \mathrm{H}), 4.72(\mathrm{~s}$, 1H), $3.78(\mathrm{~s}, 3 \mathrm{H}), 2.81(\mathrm{~s}, 3 \mathrm{H}), 1.59(\mathrm{~s}, 3 \mathrm{H}), 1.46-1.42(\mathrm{~m}, 2 \mathrm{H}), 1.27-1.24(\mathrm{~m}, 1 \mathrm{H}), 1.01-0.99(\mathrm{~m}$, $1 \mathrm{H}) ;{ }^{13} \mathrm{C}$ NMR (100 MHz, $\mathrm{CDCl}_{3}$, TMS): $\delta 170.0,158.8,144.6,139.0,138.1,133.1,132.6,130.5$, 130.1, 127.9, 126.8, 126.1, 121.0, 120.0, 113.5, 55.1, 37.6, 19.6, 4.6, 2.2; IR $\left(\mathrm{CH}_{2} \mathrm{Cl}_{2}\right): v 2971$, 2836, 1652, 1621, 1557, 1509, 1358, 1246, 1175, 1032, 905, 831, $734 \mathrm{~cm}^{-1}$; HRMS (ESI) m/z: $(\mathrm{M}+\mathrm{H})^{+}$Calcd. for $\mathrm{C}_{22} \mathrm{H}_{23} \mathrm{BrNO}_{2}$ 412.0907; found 412.0908 . 


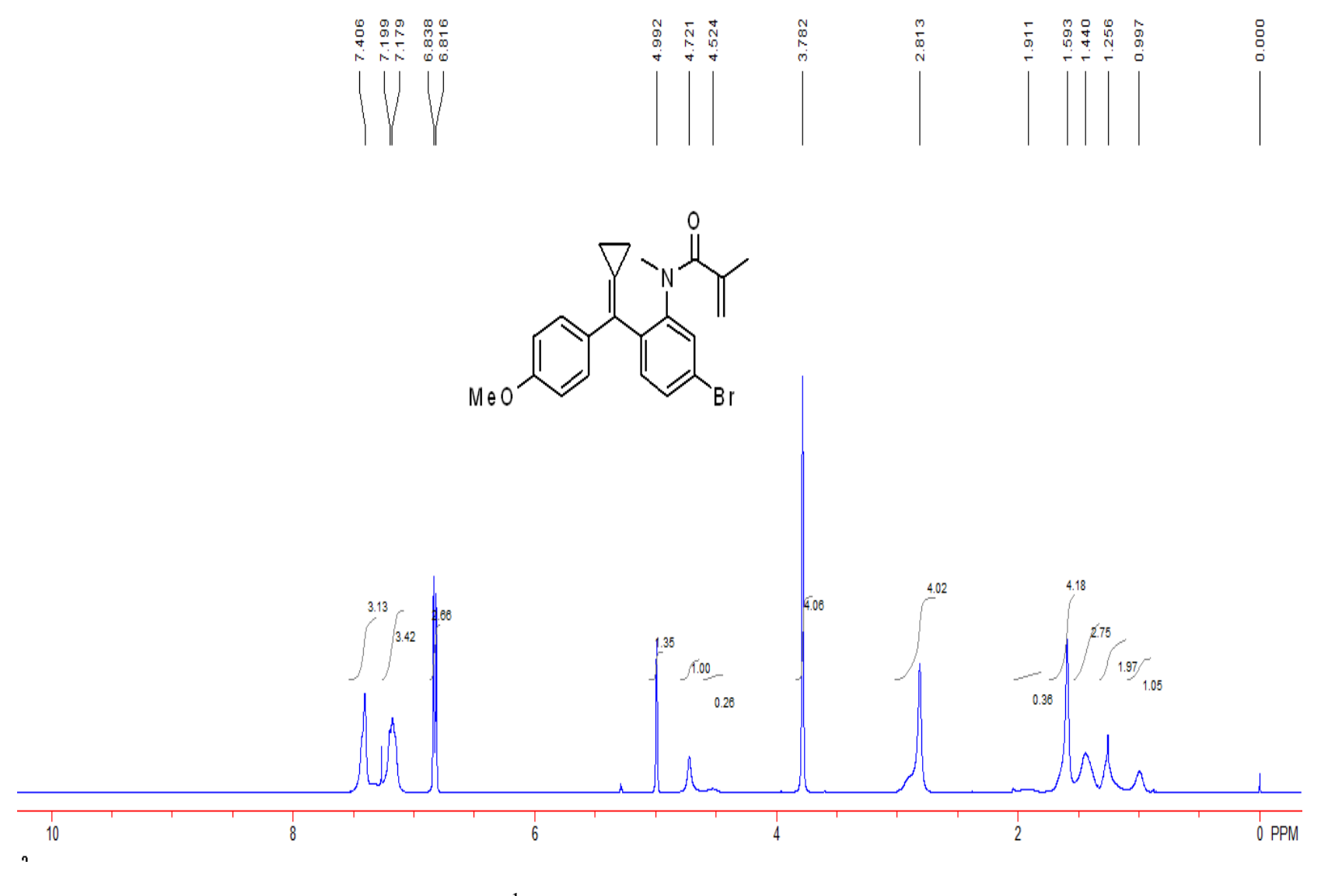

1k $\left({ }^{1} \mathrm{H}\right.$ NMR, $\left.400 \mathrm{MHz}, \mathrm{CDCl}_{3}\right)$
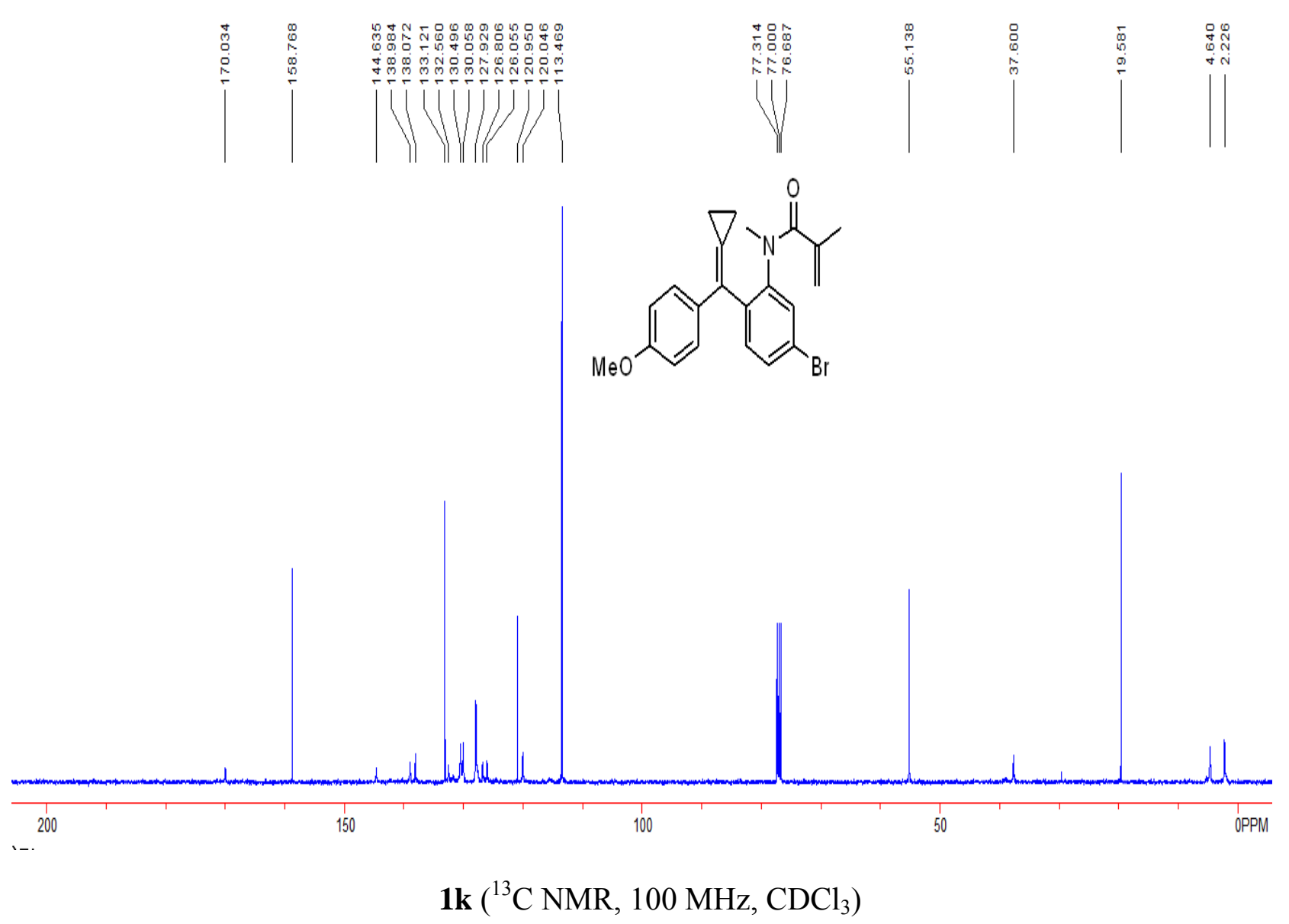


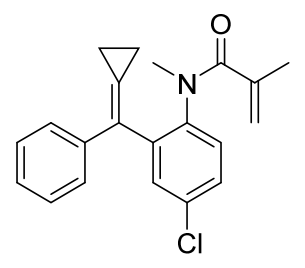

$N$-(4-chloro-2-(cyclopropylidene(phenyl)methyl)phenyl)- $N$-methylmethacrylamide (11): 534 mg, 79\% yield, white solid, m.p. $128-130{ }^{\circ} \mathrm{C}$; Eluent: PE/EA = 10/1. ${ }^{1} \mathrm{H}$ NMR (400 MHz, $\mathrm{CDCl}_{3}$, TMS): $\delta$ 7.33-7.14 (m, 8H), $4.96(\mathrm{~s}, 1 \mathrm{H}), 4.68(\mathrm{~s}, 1 \mathrm{H}), 2.71(\mathrm{~s}, 3 \mathrm{H}), 1.60(\mathrm{~s}, 3 \mathrm{H}), 1.48-1.41(\mathrm{~m}, 2 \mathrm{H})$, 1.35-1.05 (m, 2H); ${ }^{13} \mathrm{C}$ NMR (100 MHz, $\left.\mathrm{CDCl}_{3}, \mathrm{TMS}\right): \delta 169.8,141.8,140.6,139.7,138.9,132.3$, $131.4,128.8,128.2,128.0,127.2,127.1,126.7,119.7,37.3,19.5,4.5,2.3 ; \mathrm{IR}\left(\mathrm{CH}_{2} \mathrm{Cl}_{2}\right): v 3054$, 2974, 1652, 1622, 1484, 1361, 1113, 919, 773, $697 \mathrm{~cm}^{-1}$; HRMS (ESI) m/z: (M+H) Calcd. for $\mathrm{C}_{21} \mathrm{H}_{21} \mathrm{ClNO}$ 338.1306; found 338.1308. 


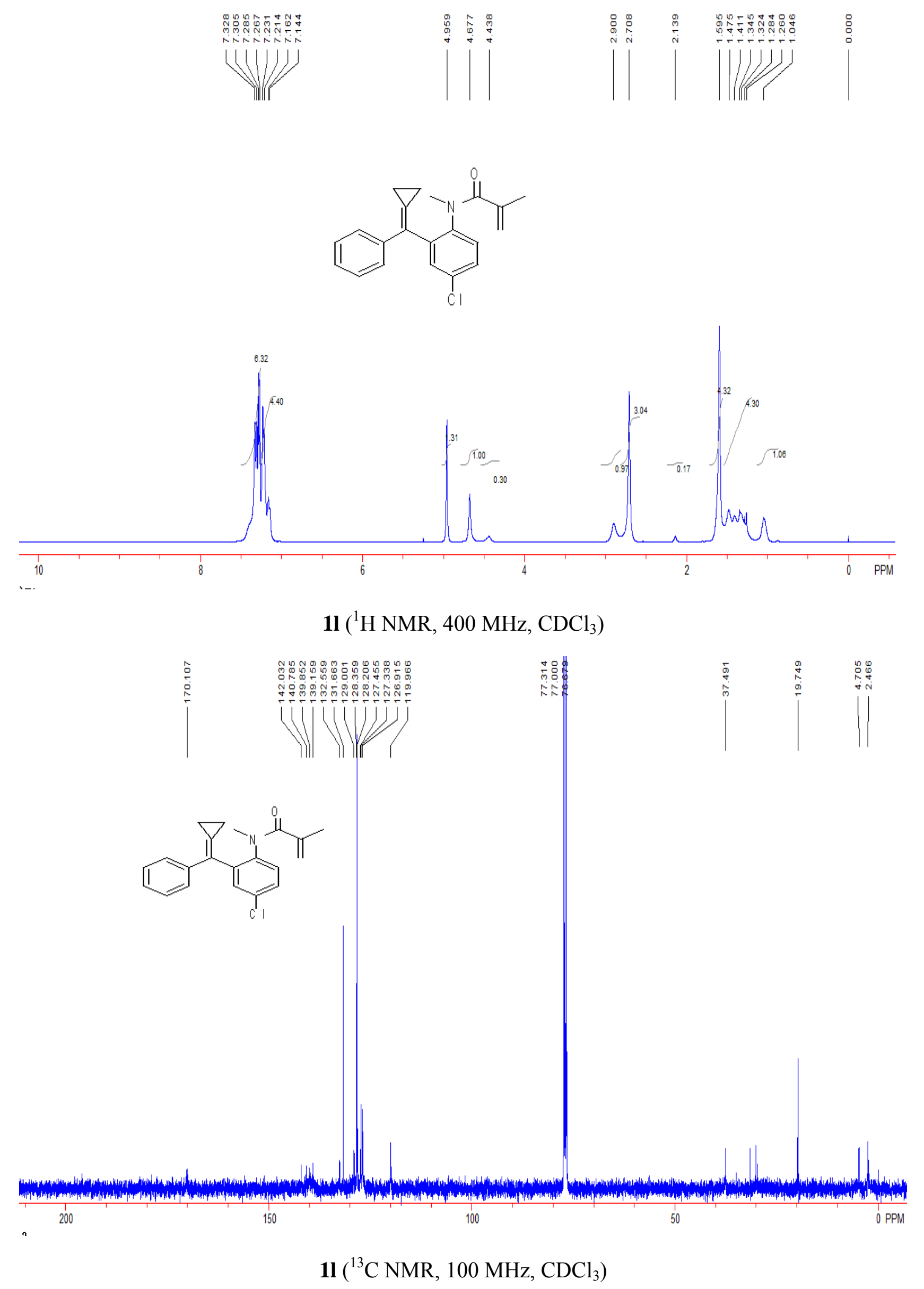




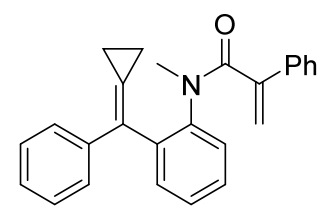

$N$-(2-(cyclopropylidene(phenyl)methyl)phenyl)- $N$-methyl-2-phenylacrylamide (1m) (two rotamers): $906 \mathrm{mg}, 66 \%$ yield, white solid, m.p. $135-137{ }^{\circ} \mathrm{C}$; Eluent: PE/EA = 10/1. ${ }^{1} \mathrm{H}$ NMR (400 $\mathrm{MHz}, \mathrm{CDCl}_{3}$, TMS): $\delta 7.49$ (d, 1.76H, $\left.J=7.6 \mathrm{~Hz}\right), 7.45-7.12(\mathrm{~m}, 12.08 \mathrm{H}), 7.02(\mathrm{t}, 1 \mathrm{H}, J=7.6 \mathrm{~Hz})$, $6.77(\mathrm{~d}, 1 \mathrm{H}, J=8.0 \mathrm{~Hz}), 5.49(\mathrm{~s}, 0.76 \mathrm{H}), 5.32(\mathrm{~s}, 1 \mathrm{H}), 5.03(\mathrm{~s}, 1 \mathrm{H}), 4.56(\mathrm{~s}, 0.76 \mathrm{H}), 2.83(\mathrm{~s}, 2.28 \mathrm{H})$, $2.73(\mathrm{~s}, 3 \mathrm{H}), 1.58(\mathrm{t}, 1.76 \mathrm{H}, J=7.6 \mathrm{~Hz}), 1.23-1.05(\mathrm{~m}, 3.52 \mathrm{H}), 0.89-0.78(\mathrm{~m}, 1.76 \mathrm{H}) ;{ }^{13} \mathrm{C} \mathrm{NMR}$ (100 MHz, $\left.\mathrm{CDCl}_{3}, \mathrm{TMS}\right): \delta 169.9,169.6,144.8,144.4,141.9,141.2,140.7,139.5,139.4,138.7$, $136.9,135.3,132.0,131.7,128.7,128.5,128.34,128.28,128.1,128.0,127.94,127.87,127.8,127.6$, $127.5,127.4,126.9,126.5,126.1,126.0,125.5,117.2,113.6,39.2,37.2,5.5,4.4,2.5,1.8$; IR $\left(\mathrm{CH}_{2} \mathrm{Cl}_{2}\right): v$ 3054, 2971, 1645, 1596, 1493, 1446, 1373, 1077, 912, 767, 751, $698 \mathrm{~cm}^{-1}$; HRMS (ESI) $\mathrm{m} / \mathrm{z}$ : $(\mathrm{M}+\mathrm{H})^{+}$Calcd. for $\mathrm{C}_{26} \mathrm{H}_{24} \mathrm{NO} 366.1852$; found 366.1853 . 

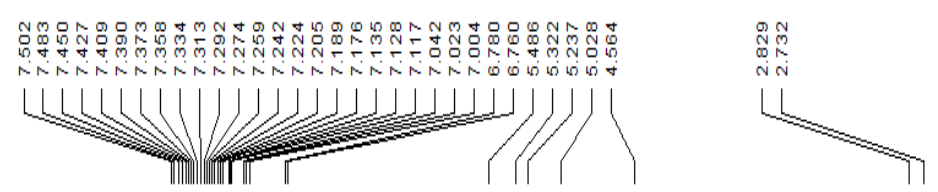

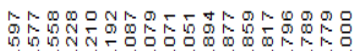

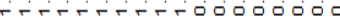
को

(IT⿱卄一二八<smiles>C=C(C(=O)N(C)c1ccccc1C(=C1CC1)c1ccccc1)c1ccccc1</smiles>

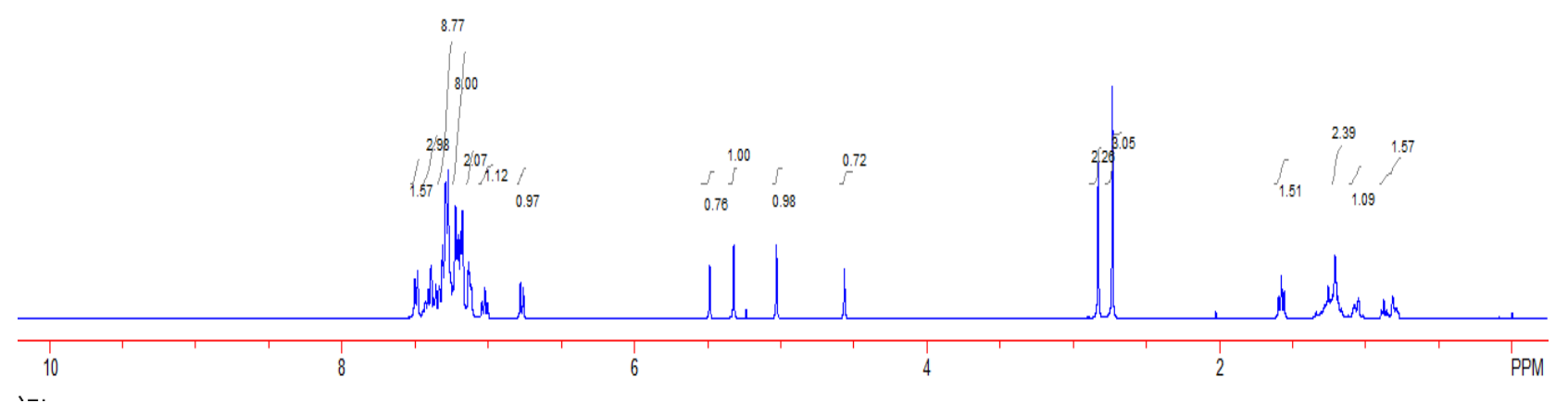

1m $\left({ }^{1} \mathrm{H} \mathrm{NMR}, 400 \mathrm{MHz}, \mathrm{CDCl}_{3}\right)$
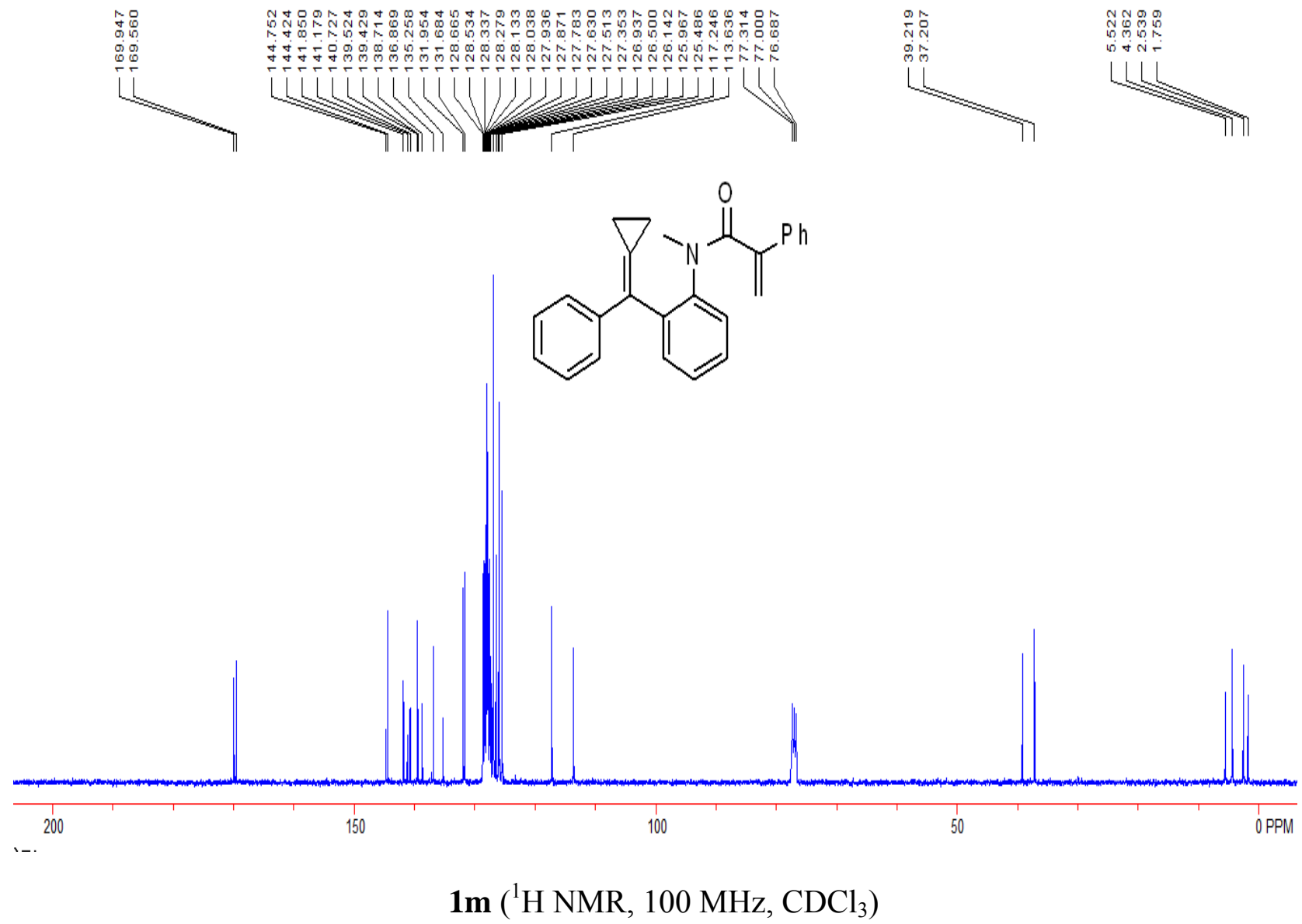


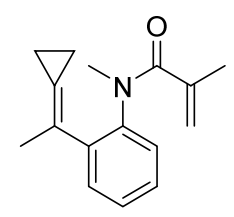

$N$-(2-(1-cyclopropylideneethyl)phenyl)- $N$-methylmethacrylamide (1n): $584 \mathrm{mg}$, 83\% yield, white solid, m.p. $51-52{ }^{\circ} \mathrm{C}$; Eluent: PE/EA = 10/1. ${ }^{1} \mathrm{H}$ NMR (400 MHz, $\left.\mathrm{CDCl}_{3}, \mathrm{TMS}\right): \delta$ 7.30-7.28 (m, 1H), 7.24-7.22 (m, 2H), 7.15-7.13 (m, 1H), $4.97(\mathrm{~s}, 1 \mathrm{H}), 4.78(\mathrm{~s}, 1 \mathrm{H}), 3.33(\mathrm{~s}, 3 \mathrm{H}), 2.09(\mathrm{~s}, 3 \mathrm{H})$, 1.67 (s, 3H), 1.25-1.05 (m, 4H); ${ }^{13} \mathrm{C}$ NMR (150 MHz, $\left.\mathrm{CDCl}_{3}, \mathrm{TMS}\right): \delta 171.0,142.1,139.6,139.4$, 130.2, 127.52, 127.46, 126.9, 124.4, 122.9, 119.8, 37.6, 21.3, 20.0, 4.3, 2.4; IR $\left(\mathrm{CH}_{2} \mathrm{Cl}_{2}\right): v 3044$, 2970, 2924, 1650, 1617, 1468, 1363, 1111, 1070, 924, 762, $589 \mathrm{~cm}^{-1}$; HRMS (ESI) m/z: (M+Na) Calcd. for $\mathrm{C}_{16} \mathrm{H}_{19} \mathrm{NONa} 264.1359$; found 264.1365. 

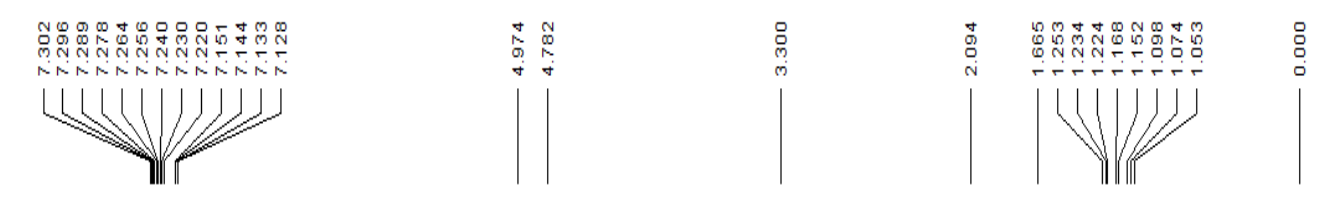<smiles>C=C(C)C(=O)n1c2cccc-2c(C)c2c1C2</smiles>

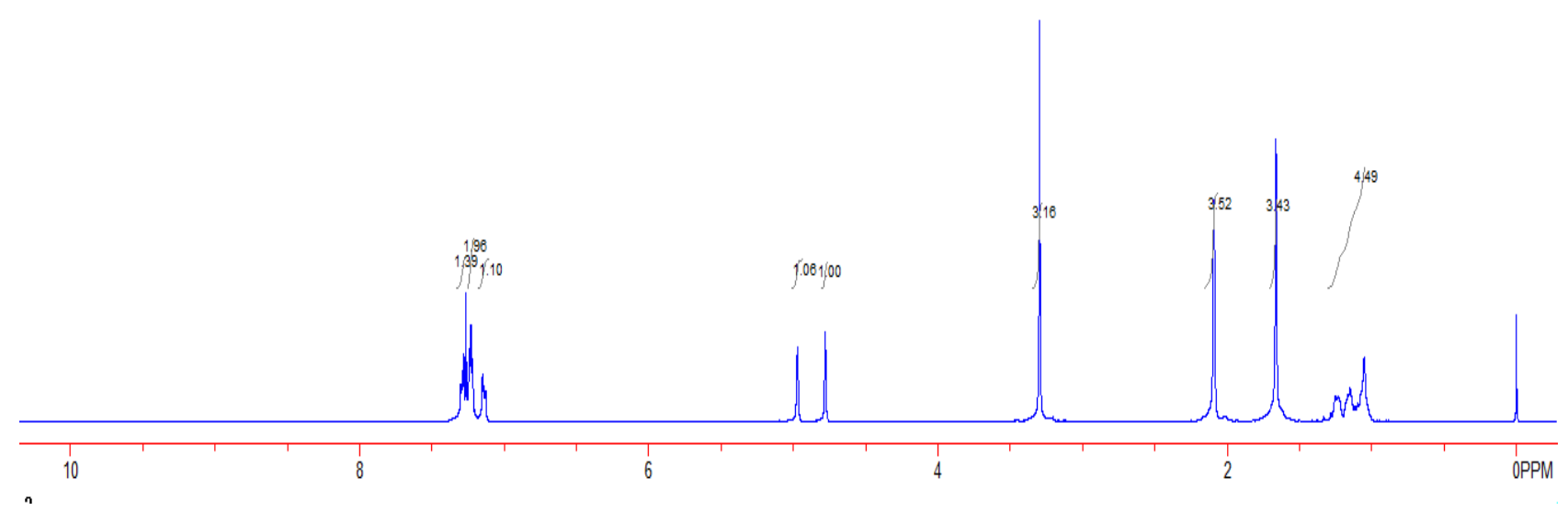
1n $\left({ }^{1} \mathrm{H} \mathrm{NMR}, 400 \mathrm{MHz}, \mathrm{CDCl}_{3}\right)$

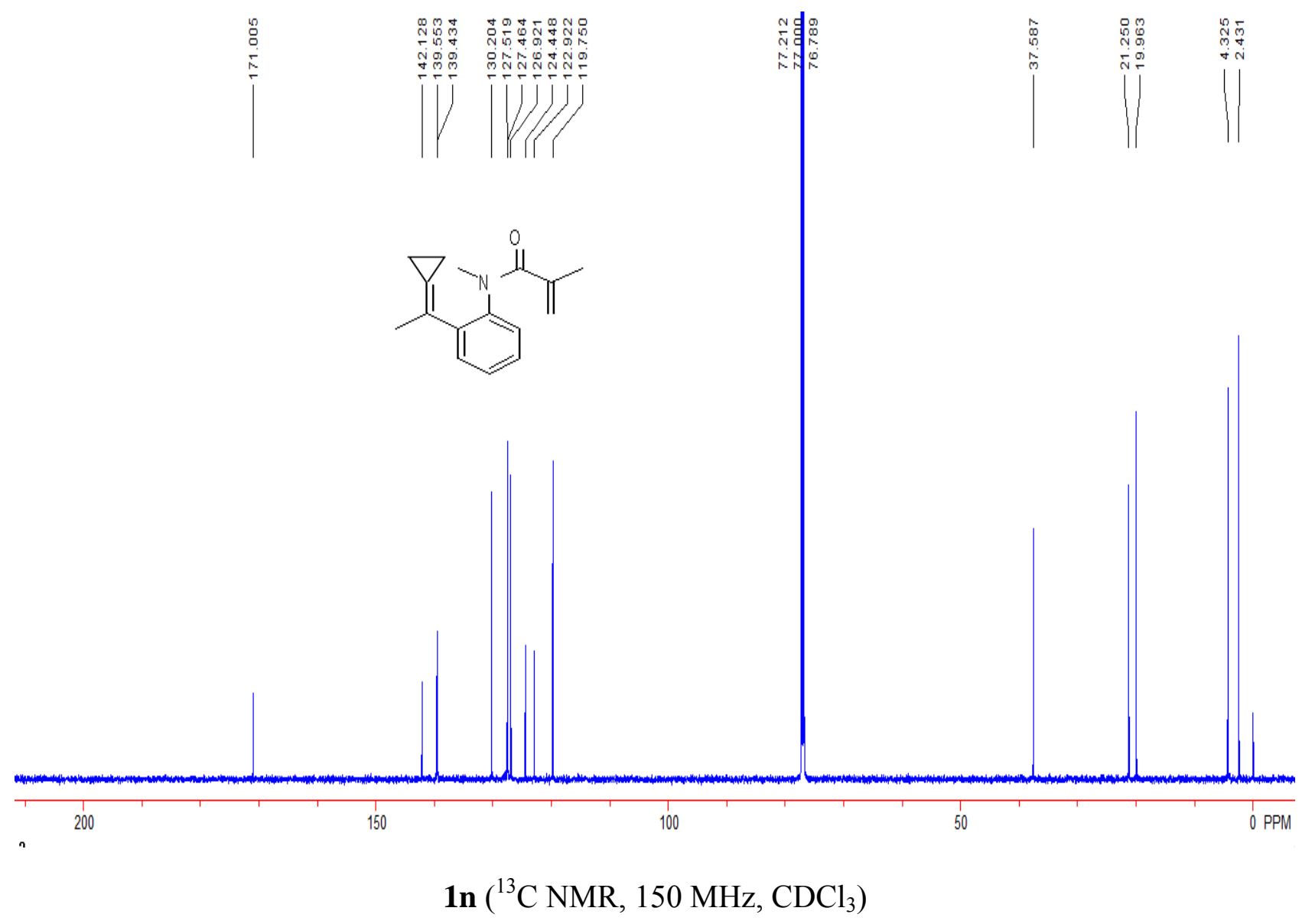




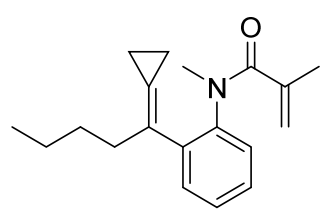

$N$-(2-(1-cyclopropylidenepentyl)phenyl)- $N$-methylmethacrylamide (10): $482 \mathrm{mg}$, 85\% yield, white solid, m.p. $56-57{ }^{\circ} \mathrm{C}$; Eluent: PE/EA = 10/1. ${ }^{1} \mathrm{H}$ NMR (400 MHz, $\left.\mathrm{CDCl}_{3}, \mathrm{TMS}\right): \delta$ 7.26-7.21 (m, 3H), 7.14-7.13 (m, 1H), 4.95 (s, 1H), 4.75 (s, 1H), 3.29 (s, 3H), 2.53-2.45 (m, 1H), 2.42-2.37 $(\mathrm{m}, 1 \mathrm{H}), 1.63(\mathrm{~s}, 3 \mathrm{H}), 1.38-1.25(\mathrm{~m}, 3 \mathrm{H}), 1.22-1.11(\mathrm{~m}, 3 \mathrm{H}), 0.93-0.84(\mathrm{~m}, 5 \mathrm{H}) ;{ }^{13} \mathrm{C}$ NMR $(150$ $\left.\mathrm{MHz}, \mathrm{CDCl}_{3}, \mathrm{TMS}\right): \delta 171.0,142.1,139.6,139.2,131.4,128.5,127.5,127.0,123.9,119.6,37.7$, 34.4, 30.6, 22.4, 20.0, 13.9, 3.2, 3.0; IR $\left(\mathrm{CH}_{2} \mathrm{Cl}_{2}\right): \vee$ 3050, 2965, 2925, 2852, 1650, 1616, 1590, 1489, 1450, 1362, 1113, 1230, 1000, 928, 774, $591 \mathrm{~cm}^{-1}$; HRMS (ESI) m/z: (M+H) Calcd. for $\mathrm{C}_{19} \mathrm{H}_{26} \mathrm{NO} 284.2009$; found 284.2013. 


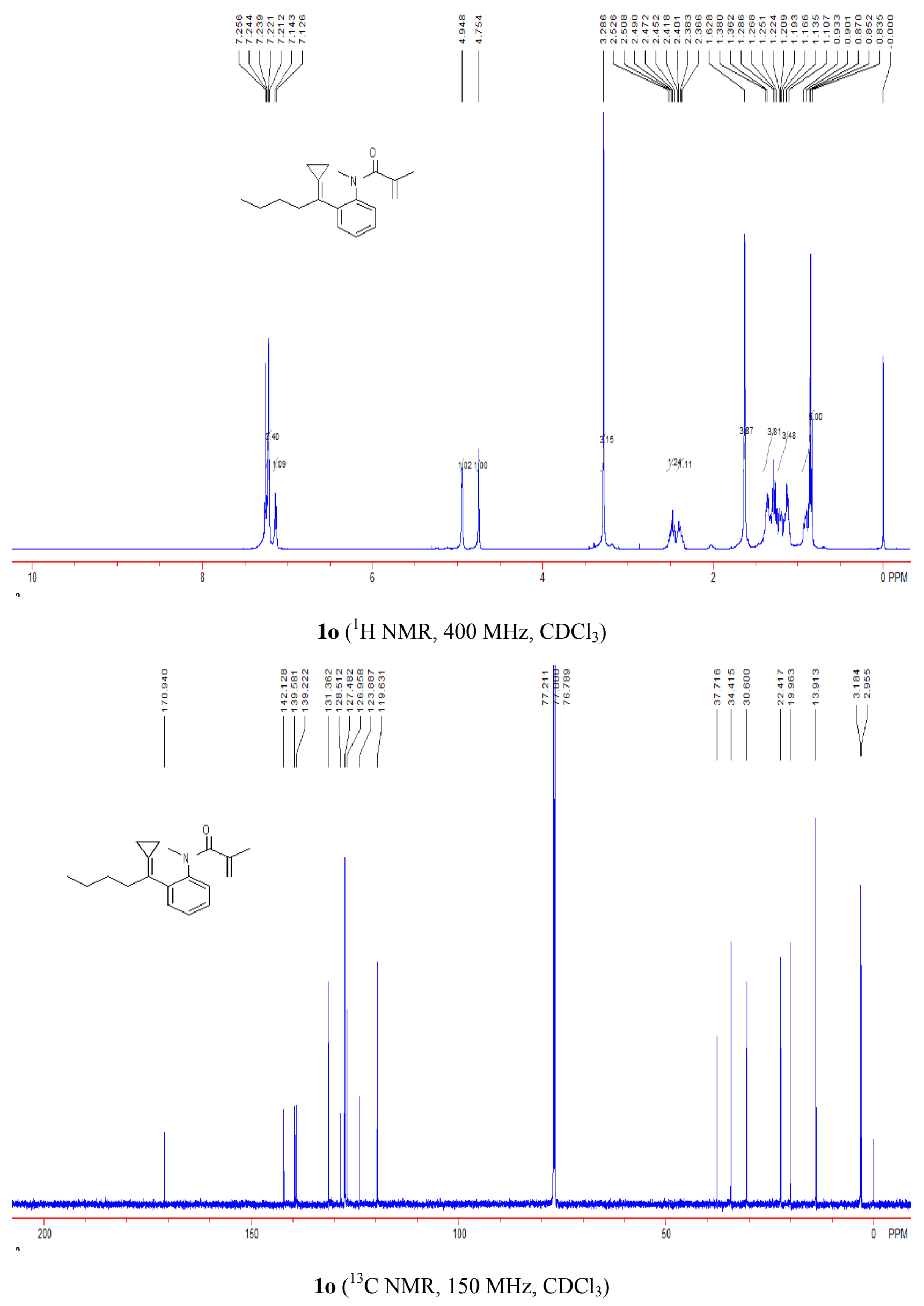




\section{Characterization Data of Products.}

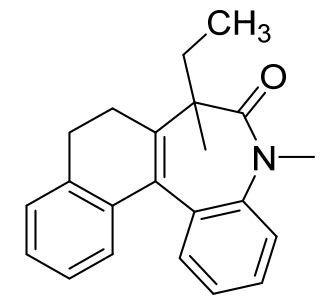

7-Ethyl-5,7-dimethyl-5,7,8,9-tetrahydro-6H-benzo[b]naphtho[1,2-d]azepin-6-one (3a)

Pale green oil, $53 \mathrm{mg}, 86 \%$ yield; Eluent: PE/EA $=10 / 1 .{ }^{1} \mathrm{H}$ NMR (400 $\left.\mathrm{MHz}, \mathrm{CDCl}_{3}, \mathrm{TMS}\right) \delta$ 7.37-7.33 (m, 1H), 7.31-7.25 (m, 2H), 7.20-7.17 (m, 1H), 7.15-7.08 (m, 3H), 6.96 (d, J = 7.6 Hz, $1 \mathrm{H}), 3.43(\mathrm{~s}, 3 \mathrm{H}), 2.84-2.68(\mathrm{~m}, 3 \mathrm{H}), 2.25\left(\mathrm{td}, J_{1}=6.0 \mathrm{~Hz}, J_{2}=16.4 \mathrm{~Hz}, 1 \mathrm{H}\right), 1.51(\mathrm{~s}, 3 \mathrm{H})$, 1.14-1.04 (m, 2H), $0.60(\mathrm{t}, J=7.2 \mathrm{~Hz}, 3 \mathrm{H}) ;{ }^{13} \mathrm{C} \mathrm{NMR}\left(150 \mathrm{MHz}, \mathrm{CDCl}_{3}, \mathrm{TMS}\right) \delta 174.4,143.5$, $142.3,136.6,136.5,132.5,131.9,130.0,127.9,126.8,126.7,126.0,123.5,121.3,51.3,37.8,28.6$, 26.7, 26.2, 22.7, 9.7; IR $\left(\mathrm{CH}_{2} \mathrm{Cl}_{2}\right): v 2959,2920,1659,1394,1250,1070,1057,806,779,766,729$, $670 \mathrm{~cm}^{-1}$; HRMS (ESI) m/z: (M+H) Calcd. for $\mathrm{C}_{22} \mathrm{H}_{24} \mathrm{NO} 318.1852$; found 318.1847. 


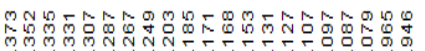

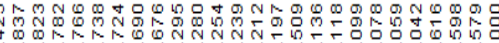

LLMMWUWJJJJ

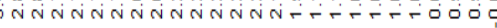

- IIII

แWUHUH LUHU
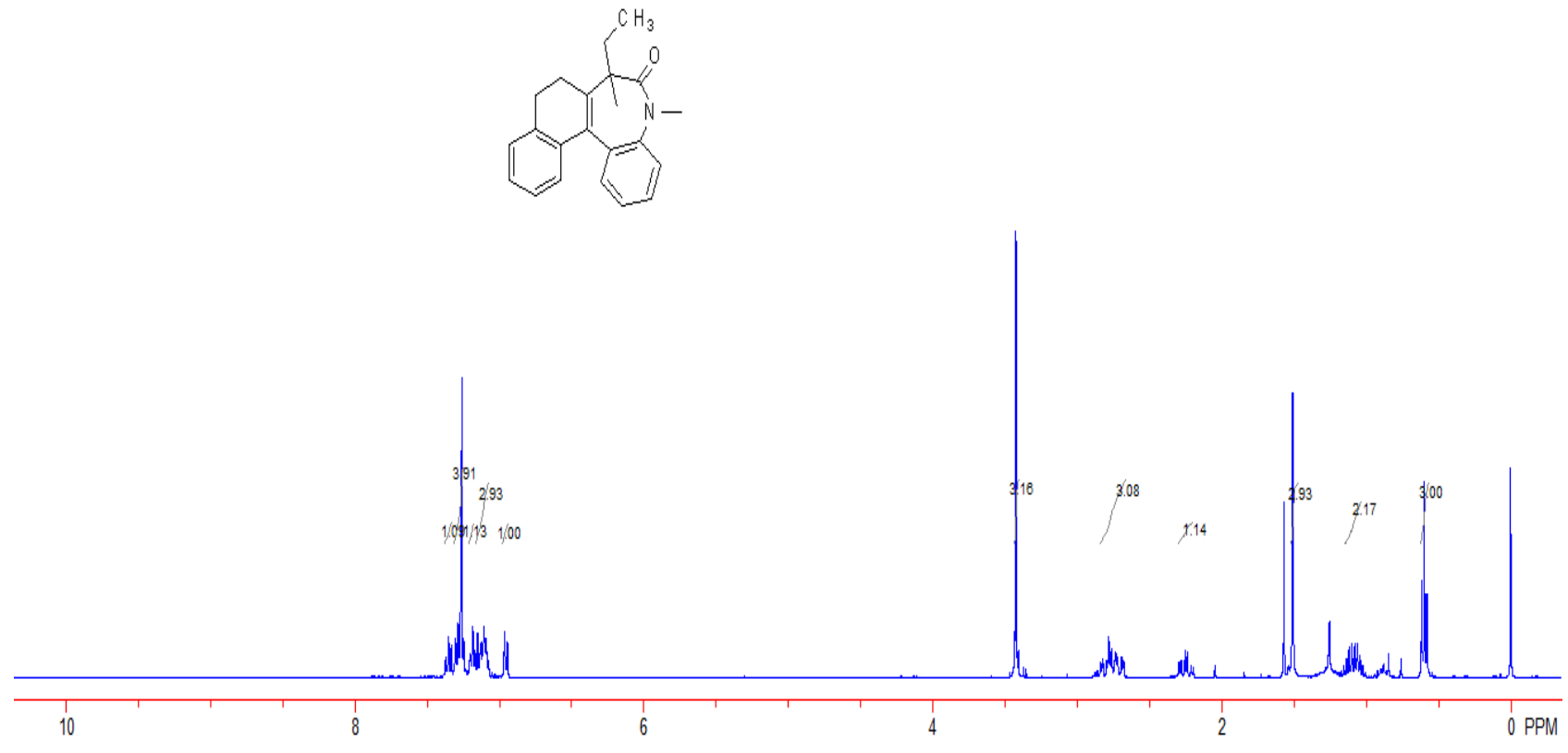

3a $\left({ }^{1} \mathrm{H}\right.$ NMR, $\left.400 \mathrm{MHz}, \mathrm{CDCl}_{3}\right)$

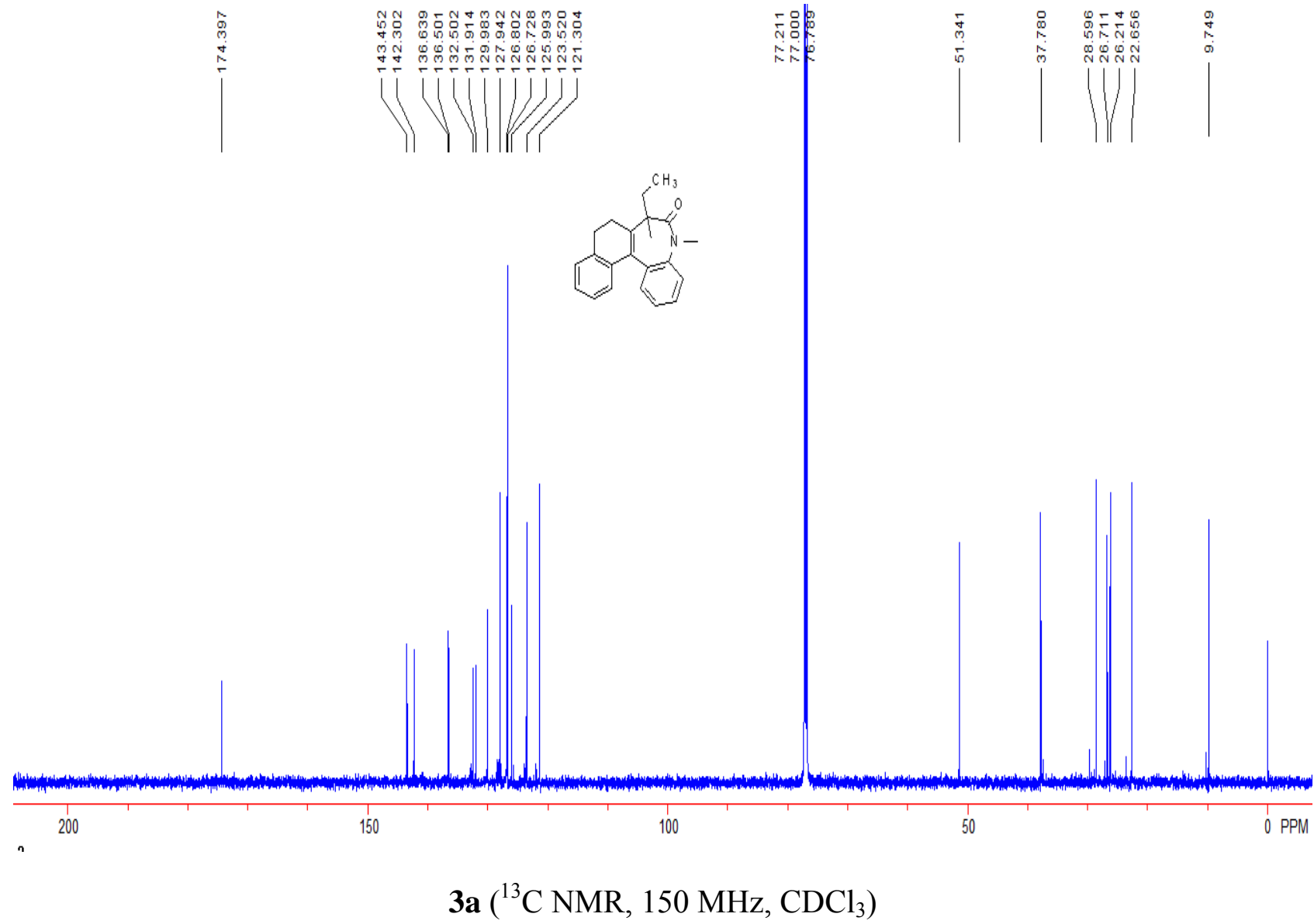




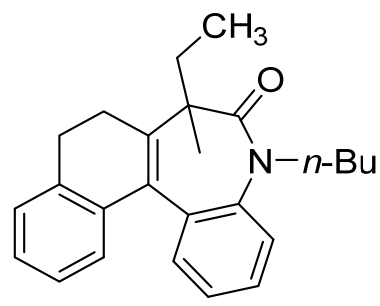

5-Butyl-7-ethyl-5,7-dimethyl-5,7,8,9-tetrahydro-6H-benzo[b]naphtho[1,2-d]azepin-6-one (3b)

Pale green oil, $66 \mathrm{mg}, 92 \%$ yield; Eluent: PE/EA $=10 / 1 .{ }^{1} \mathrm{H}$ NMR (400 $\left.\mathrm{MHz}, \mathrm{CDCl}_{3}, \mathrm{TMS}\right) \delta$ 7.36-7.32 (m, 2H), 7.28-7.26 (m, 1H), 7.20-7.09 (m, 4H), 6.94 (d, J = 7.2 Hz, 1H), 4.32-4.25 (m, $1 \mathrm{H}), 3.68-3.62(\mathrm{~m}, 1 \mathrm{H}), 2.86-2.66(\mathrm{~m}, 3 \mathrm{H}), 2.24\left(\mathrm{td}, J_{1}=6.4 \mathrm{~Hz}, J_{2}=16.4 \mathrm{~Hz}, 1 \mathrm{H}\right), 1.50-1.37(\mathrm{~m}$, $5 \mathrm{H}), 1.16-1.02(\mathrm{~m}, 4 \mathrm{H}), 0.75(\mathrm{t}, J=7.2 \mathrm{~Hz}, 3 \mathrm{H}), 0.61(\mathrm{t}, J=7.2 \mathrm{~Hz}, 3 \mathrm{H}) ;{ }^{13} \mathrm{C} \mathrm{NMR}(100 \mathrm{MHz}$, $\left.\mathrm{CDCl}_{3}, \mathrm{TMS}\right) \delta 173.2,143.8,141.1,136.7,136.5,133.9,132.0,129.9,127.9,126.7,126.6,126.0$, 123.8, 122.2, 51.5, 49.6, 30.0, 28.6, 26.6, 26.1, 22.7, 19.9, 13.7, 9.8; IR $\left(\mathrm{CH}_{2} \mathrm{Cl}_{2}\right): v$ 2988, 1644, 1493, 1450, 1379, 1248, 1231, 1051, 766, 750, $669 \mathrm{~cm}^{-1}$; HRMS (ESI) m/z: $(\mathrm{M}+\mathrm{H})^{+}$Calcd. for $\mathrm{C}_{25} \mathrm{H}_{30} \mathrm{NO} 360.2322$; found 360.2311 . 

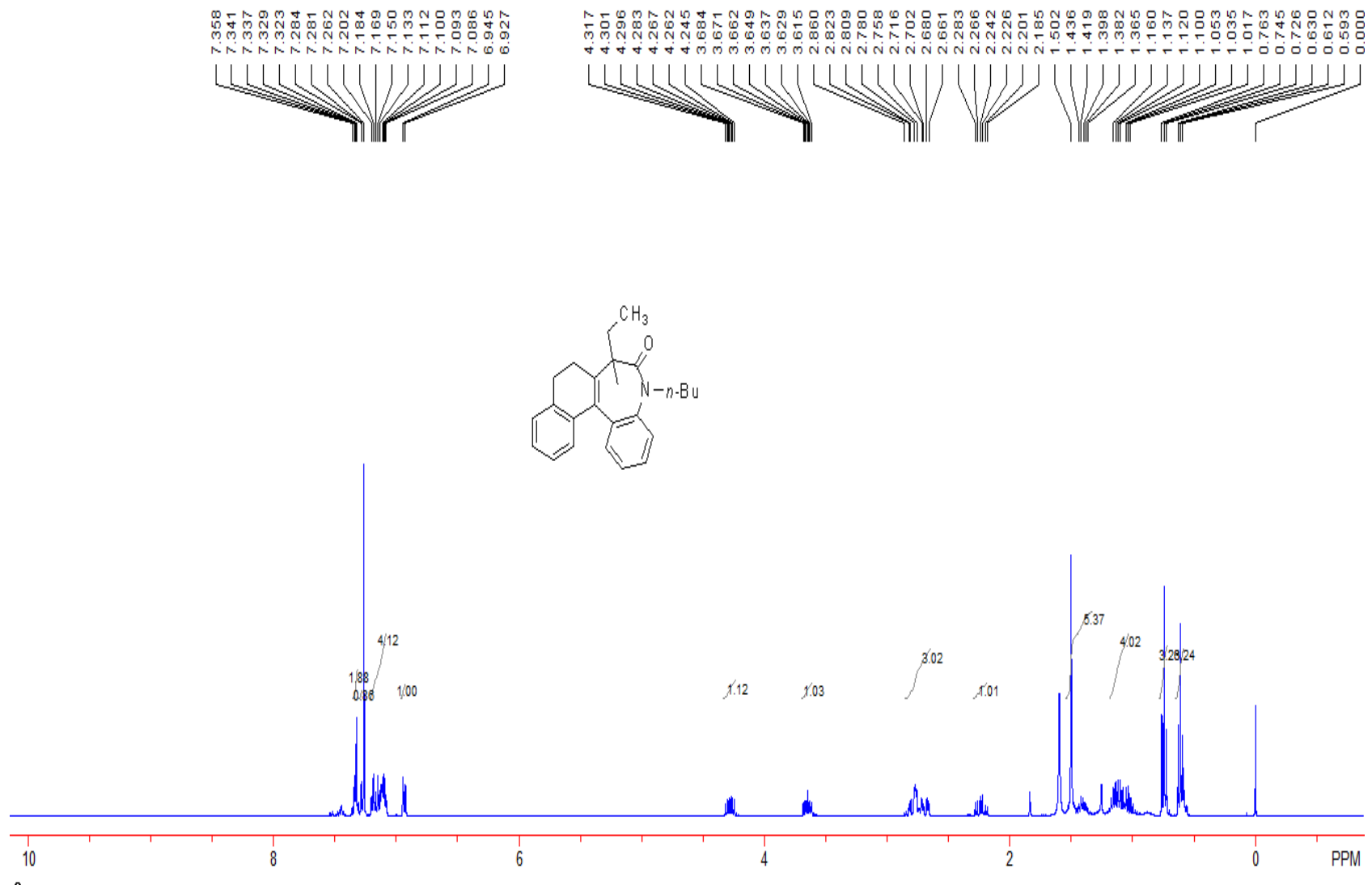

3b $\left({ }^{1} \mathrm{H}\right.$ NMR, $\left.400 \mathrm{MHz}, \mathrm{CDCl}_{3}\right)$
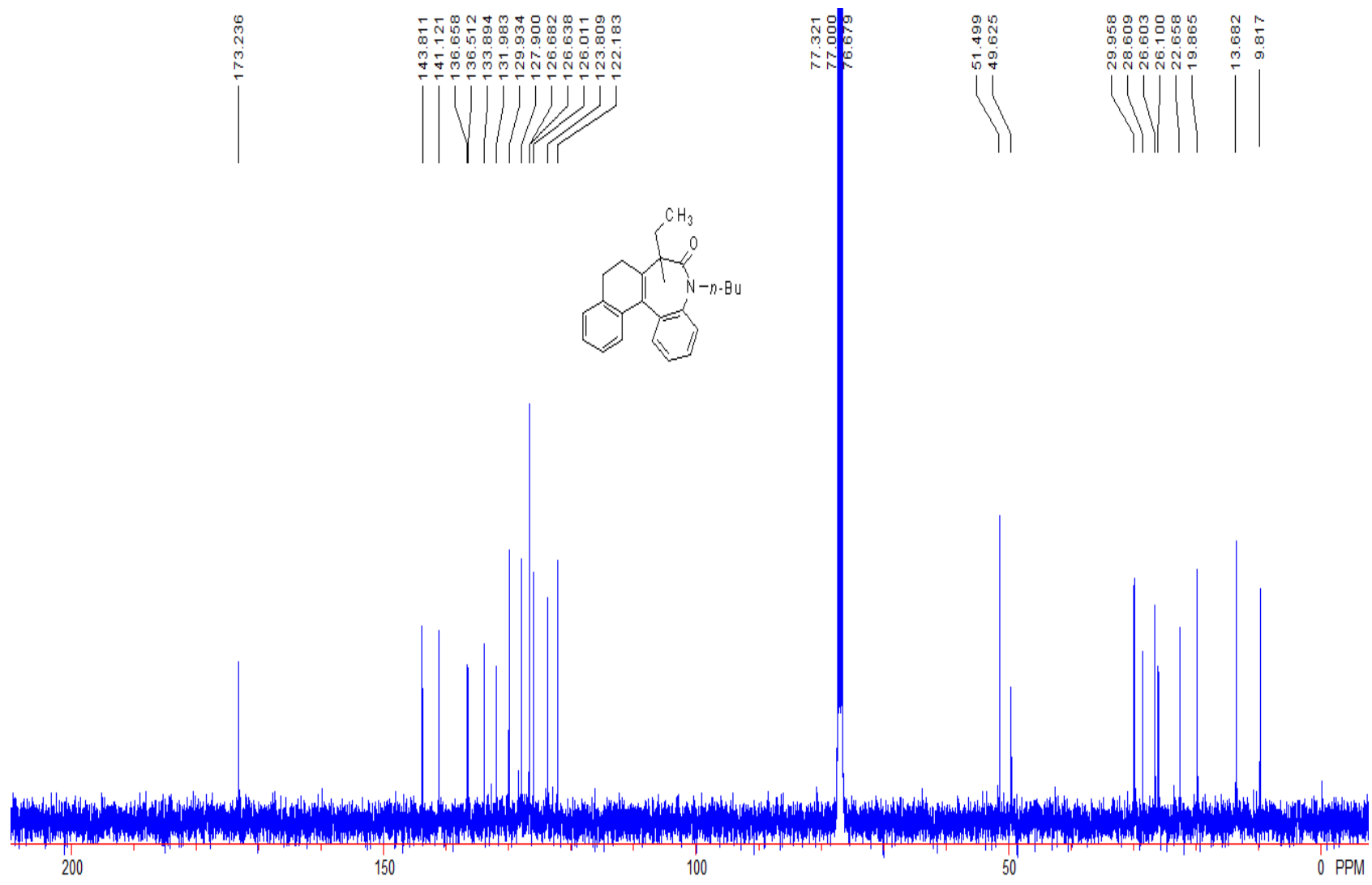

3b $\left({ }^{13} \mathrm{C} \mathrm{NMR}, 100 \mathrm{MHz}, \mathrm{CDCl}_{3}\right)$ 


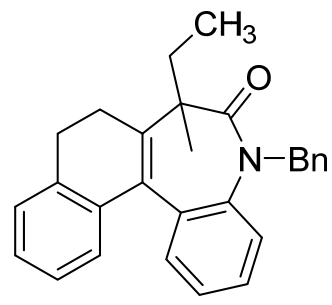

5-Benzyl-7-ethyl-7-methyl-5,7,8,9-tetrahydro-6H-benzo[b]naphtho[1,2-d]azepin-6-one (3c)

Pale green oil, $71 \mathrm{mg}, 90 \%$ yield; Eluent: $\mathrm{PE} / \mathrm{EA}=10 / 1 .{ }^{1} \mathrm{H}$ NMR $\left(400 \mathrm{MHz}, \mathrm{CDCl}_{3}, \mathrm{TMS}\right) \delta$ 7.31-7.29 (m, 1H), 7.25-7.24 (m, 2H), 7.23-7.18 (m, 2H), 7.17-7.13 (m, 1H), 7.08-7.06 (m, 3H), 7.04-7.01 (m, 3H), 5.52 (d, $J=15.6 \mathrm{~Hz}, 1 \mathrm{H}), 4.92$ (d, $J=15.6 \mathrm{~Hz}, 1 \mathrm{H}), 2.84-2.70(\mathrm{~m}, 3 \mathrm{H}), 2.27$ (td, $\left.J_{1}=6.4 \mathrm{~Hz}, J_{2}=16.0 \mathrm{~Hz}, 1 \mathrm{H}\right), 1.55(\mathrm{~s}, 3 \mathrm{H}), 1.24-1.21(\mathrm{~m}, 1 \mathrm{H}), 1.14-1.08(\mathrm{~m}, 1 \mathrm{H}), 0.67(\mathrm{t}, J=7.6$ $\mathrm{Hz}, 3 \mathrm{H}) ;{ }^{13} \mathrm{C} \mathrm{NMR}\left(150 \mathrm{MHz}, \mathrm{CDCl}_{3}\right.$, TMS) $\delta$ 173.7, 143.4 140.9, 137.9, 136.6, 136.3, 134.0, $132.3,129.9,128.4,127.9,127.0,126.7,126.6,125.9,124.0,122.0,52.7,51.6,28.6,26.7,26.2$, 22.7, 9.9; IR $\left(\mathrm{CH}_{2} \mathrm{Cl}_{2}\right): v$ 2919, 2850, 1649, 1494, 1447, 1378, 1249, 1079, 1066, 1058, 767, 697, $669 \mathrm{~cm}^{-1}$; HRMS (ESI) m/z: $(\mathrm{M}+\mathrm{H})^{+}$Calcd. for $\mathrm{C}_{28} \mathrm{H}_{28} \mathrm{NO} 394.2165$; found 394.2161. 


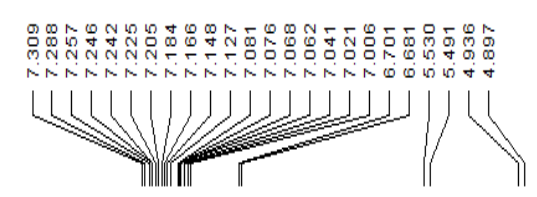

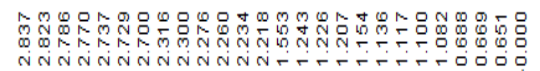

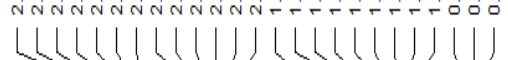

WW
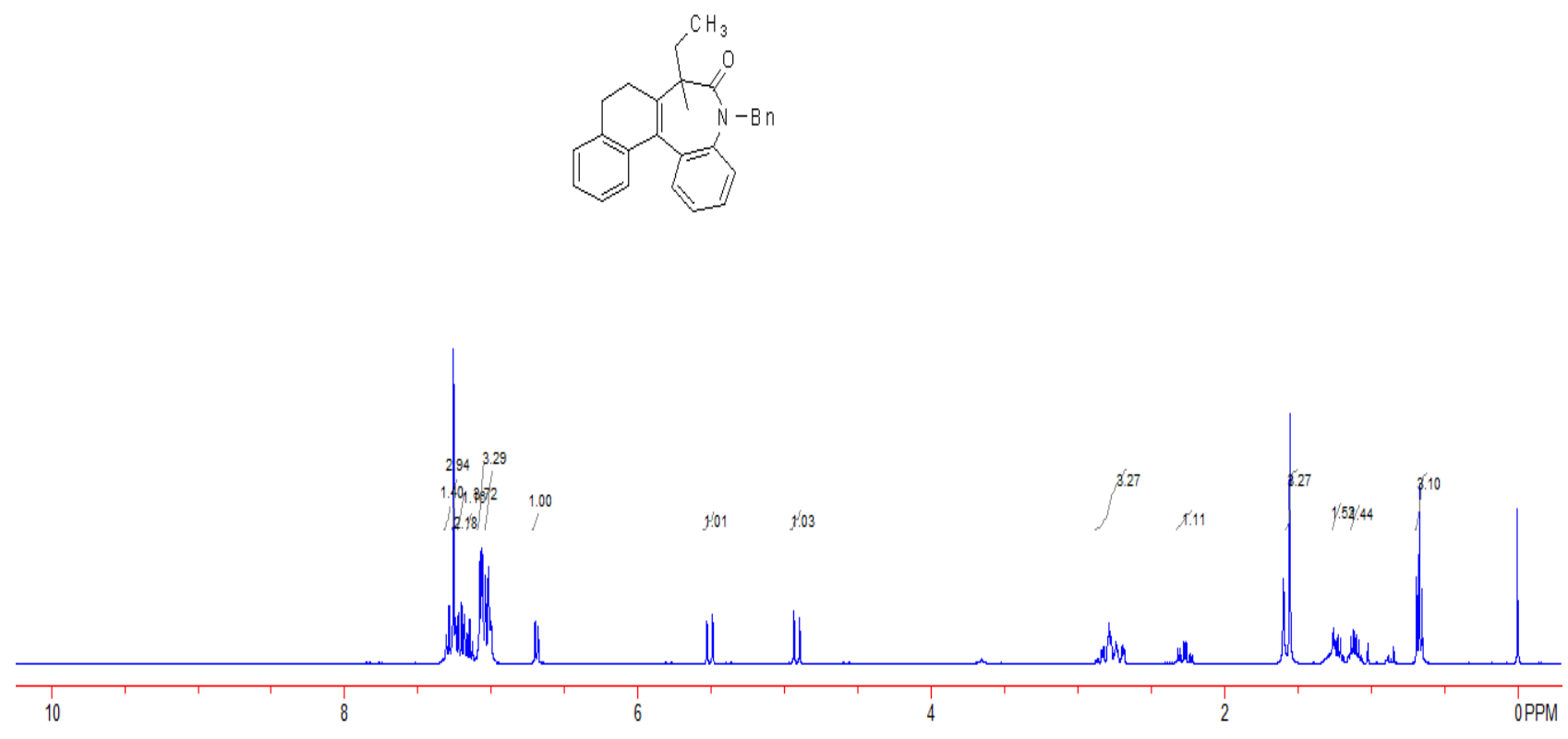

3c $\left({ }^{1} \mathrm{H}\right.$ NMR, $\left.400 \mathrm{MHz}, \mathrm{CDCl}_{3}\right)$
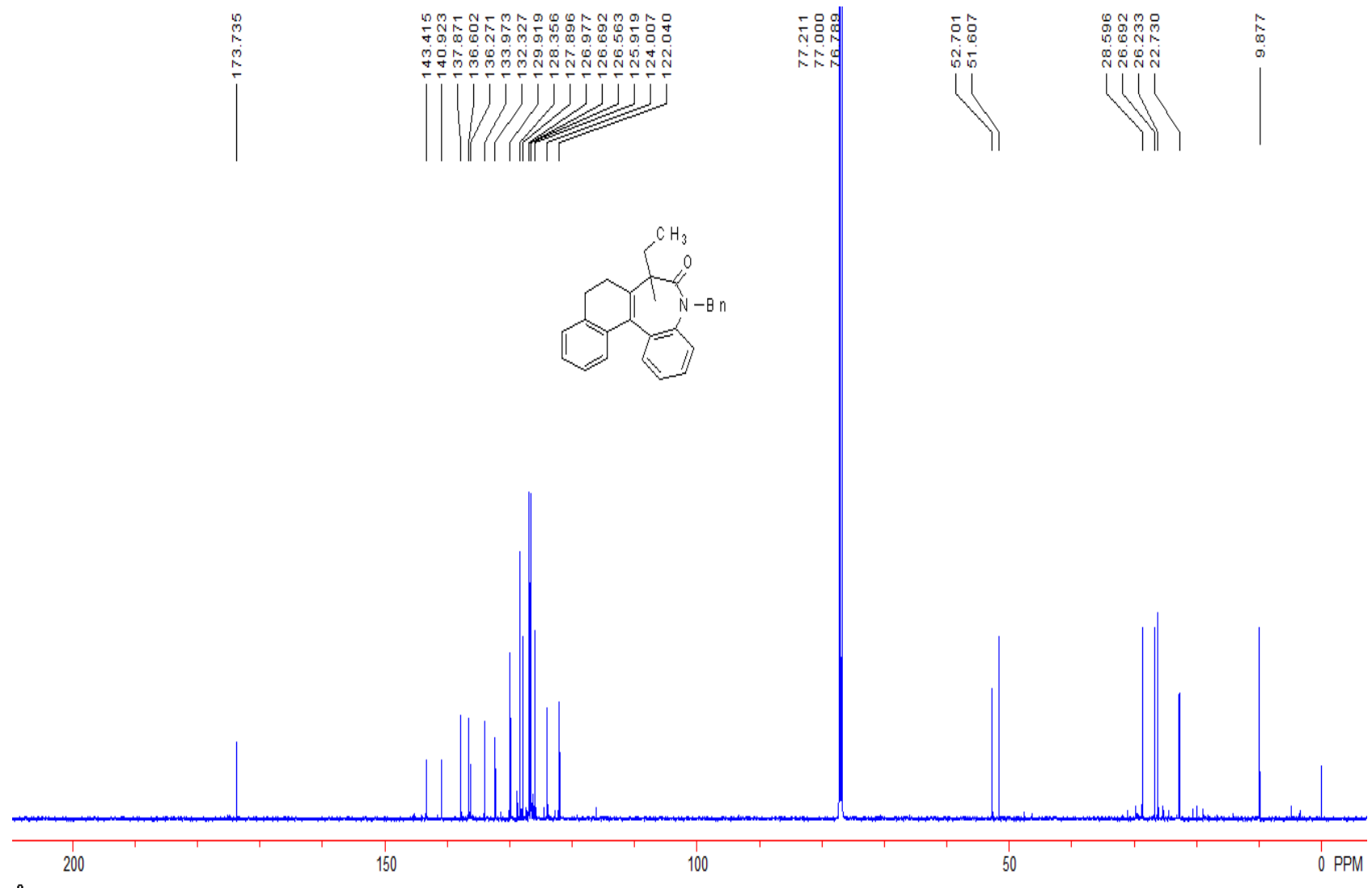

3c $\left({ }^{13} \mathrm{C} \mathrm{NMR,} 150 \mathrm{MHz}, \mathrm{CDCl}_{3}\right)$ 


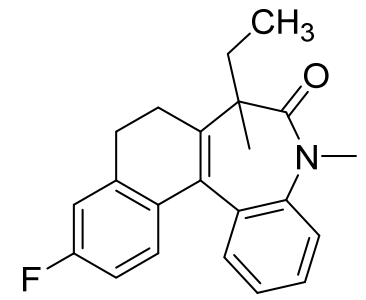

7-Ethyl-11-fluoro-5,7-dimethyl-5,7,8,9-tetrahydro-6H-benzo[b]naphtho[1,2-d]azepin-6-one (3d)

Pale green oil, $54 \mathrm{mg}, 85 \%$ yield; Eluent: PE/EA $=10 / 1 .{ }^{1} \mathrm{H}$ NMR (400 $\left.\mathrm{MHz}, \mathrm{CDCl}_{3}, \mathrm{TMS}\right) \delta$ 7.38-7.33 (m, 1H), 7.27-7.25 (m, 2H), 7.13-7.09 (m, 1H), 6.94-6.89 (m, 2H), 6.80-6.75 (m, 1H), $3.43(\mathrm{~s}, 3 \mathrm{H}), 2.85-2.68(\mathrm{~m}, 3 \mathrm{H}), 2.15\left(\mathrm{td}, J_{1}=5.6 \mathrm{~Hz}, J_{2}=16.4 \mathrm{~Hz}, 1 \mathrm{H}\right), 1.50(\mathrm{~s}, 3 \mathrm{H}), 1.14-1.01(\mathrm{~m}$, 2H), 0.59 (t, $J=7.2 \mathrm{~Hz}, 3 \mathrm{H}) ;{ }^{13} \mathrm{C} \mathrm{NMR}\left(100 \mathrm{MHz}, \mathrm{CDCl}_{3}, \mathrm{TMS}\right) \delta 174.4,161.5$ (d, $J=245.0 \mathrm{~Hz}$ ), 142.59, 142.57, 142.3, 139.2 (d, $J=8.0 \mathrm{~Hz}), 132.7$ (d, $J=3.6 \mathrm{~Hz}), 132.4,131.2,129.8,128.5$ (d, $J$ $=8.1 \mathrm{~Hz}), 128.1,123.6,121.4,113.7(\mathrm{~d}, J=21.9 \mathrm{~Hz}), 112.5(\mathrm{~d}, J=21.1 \mathrm{~Hz}), 51.3,37.8,28.8,26.7$, 26.0, 22.7, 9.8; ${ }^{19} \mathrm{~F}$ NMR (376 MHz, $\left.\mathrm{CDCl}_{3}\right): \delta$-115.7; IR $\left(\mathrm{CH}_{2} \mathrm{Cl}_{2}\right): v$ 2981, 1647, 1599, 1488, 1356, 1258, 1090, 1059, 889, 871, 771, 741, 721, $669 \mathrm{~cm}^{-1}$; HRMS (ESI) m/z: $(\mathrm{M}+\mathrm{H})^{+}$Calcd. for $\mathrm{C}_{22} \mathrm{H}_{23} \mathrm{FNO} 336.1758$; found 336.1751 . 


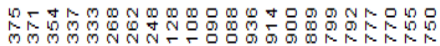

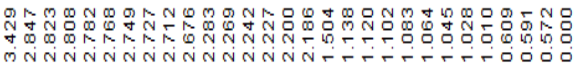

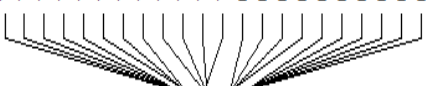

NAN

nो $1 / 1 / \pi$

WIIf
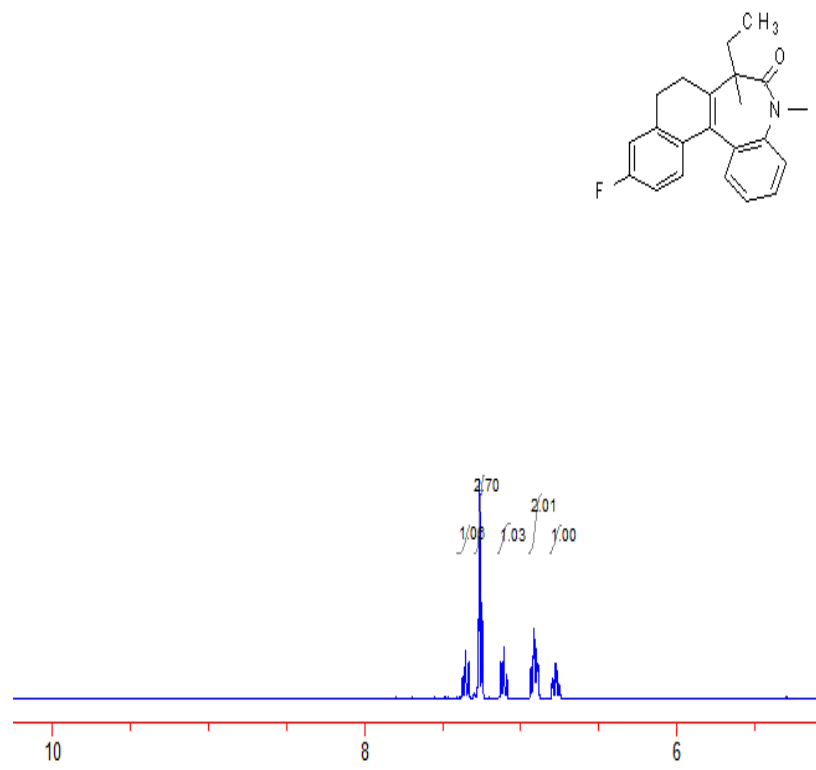

T

3d $\left({ }^{1} \mathrm{H}\right.$ NMR, $\left.400 \mathrm{MHz}, \mathrm{CDCl}_{3}\right)$
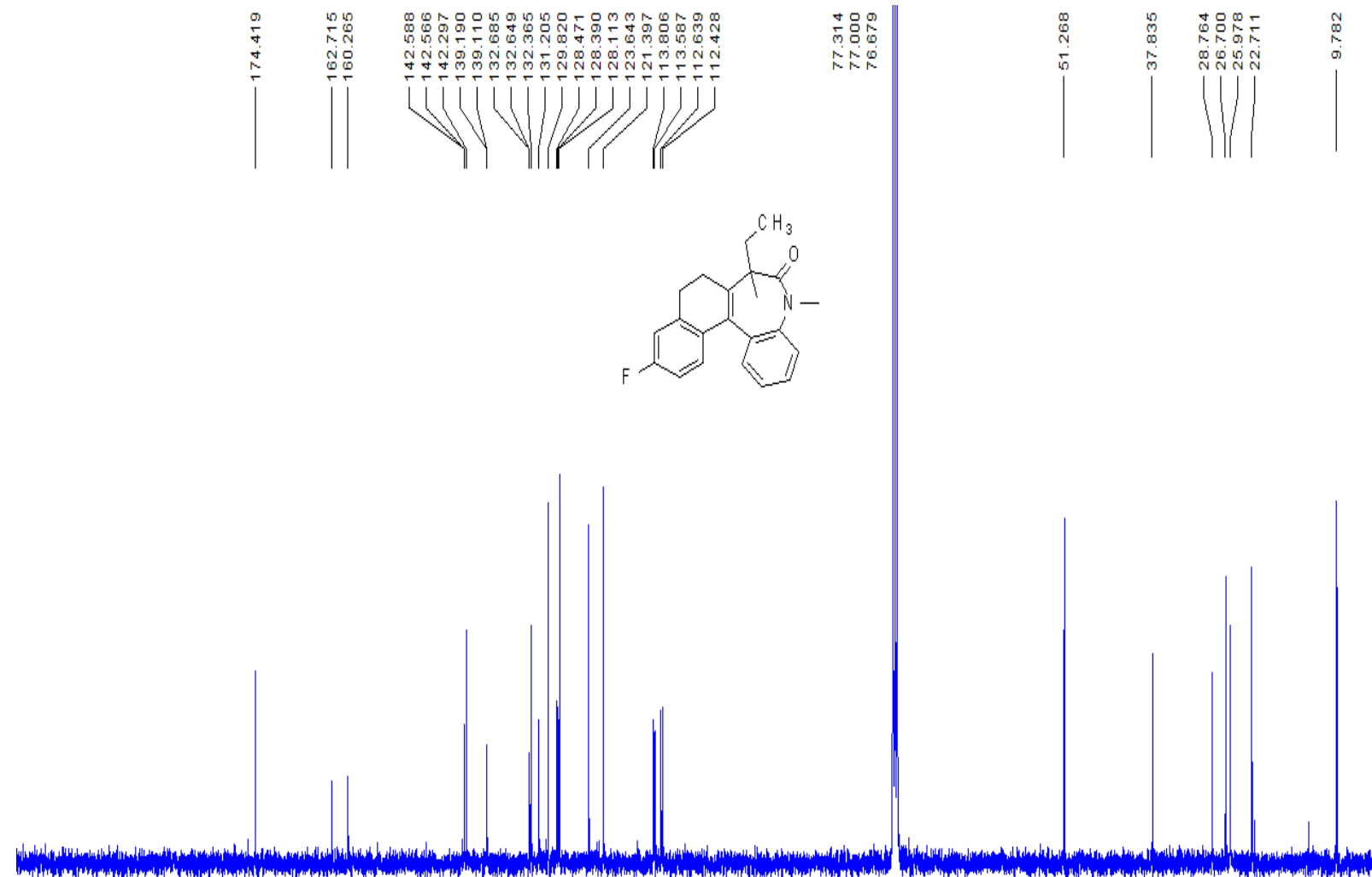

200

150
100

3d $\left({ }^{13} \mathrm{C} \mathrm{NMR}, 100 \mathrm{MHz}, \mathrm{CDCl}_{3}\right)$ 

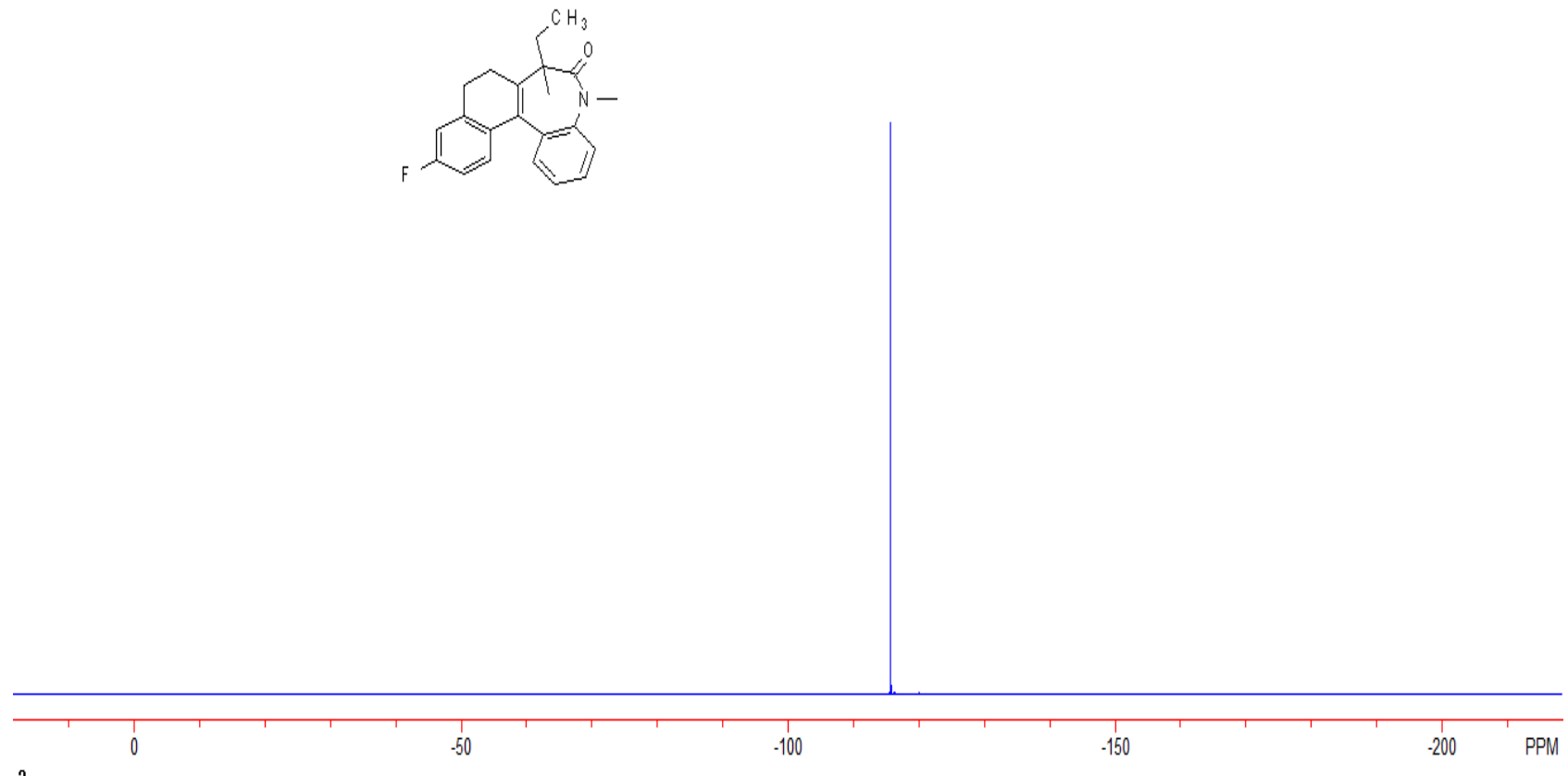

3d $\left({ }^{19} \mathrm{~F}\right.$ NMR, $\left.376 \mathrm{MHz}, \mathrm{CDCl}_{3}\right)$ 


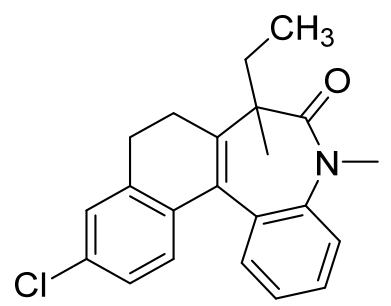

7-Ethyl-11-chloro-5,7-dimethyl-5,7,8,9-tetrahydro-6H-benzo[b]naphtho[1,2-d]azepin-6-one (3e)

Pale green oil, $58 \mathrm{mg}, 82 \%$ yield; Eluent: $\mathrm{PE} / \mathrm{EA}=10 / 1 .{ }^{1} \mathrm{H}$ NMR $\left(400 \mathrm{MHz}, \mathrm{CDCl}_{3}, \mathrm{TMS}\right) \delta$ 7.38-7.34 (m, 1H), 7.27-7.23 (m, 2H), 7.18 (d, J=1.2 Hz, 1H), 7.13-7.11 (m, 1H), 7.09-7.05 (m, $1 \mathrm{H}), 6.88(\mathrm{~d}, J=8.4 \mathrm{~Hz}, 1 \mathrm{H}), 3.43(\mathrm{~s}, 3 \mathrm{H}), 2.85-2.68(\mathrm{~m}, 3 \mathrm{H}), 2.28-2.18(\mathrm{~m}, 1 \mathrm{H}), 1.50(\mathrm{~s}, 3 \mathrm{H})$, 1.14-1.01 (m, 2H), $0.59(\mathrm{t}, J=7.2 \mathrm{~Hz}, 3 \mathrm{H}) ;{ }^{13} \mathrm{C} \mathrm{NMR}\left(100 \mathrm{MHz}, \mathrm{CDCl}_{3}, \mathrm{TMS}\right) \delta 174.3,143.7$, $142.3,138.5,135.0,132.08,132.06,131.2,129.7,128.2,128.1,126.7,126.0,123.6,121.4,51.4$, 37.8, 28.4, 26.7, 26.0, 22.6, 9.7; IR $\left(\mathrm{CH}_{2} \mathrm{Cl}_{2}\right):$ v 2954, 1644, 1596, 1499, 1445, 1381, 1256, 1254, 1089, 877, 823, 759, 721, $675 \mathrm{~cm}^{-1}$; HRMS (ESI) m/z: $(\mathrm{M}+\mathrm{H})^{+}$Calcd. for $\mathrm{C}_{22} \mathrm{H}_{23} \mathrm{ClNO} 352.1463$; found 352.1454 . 

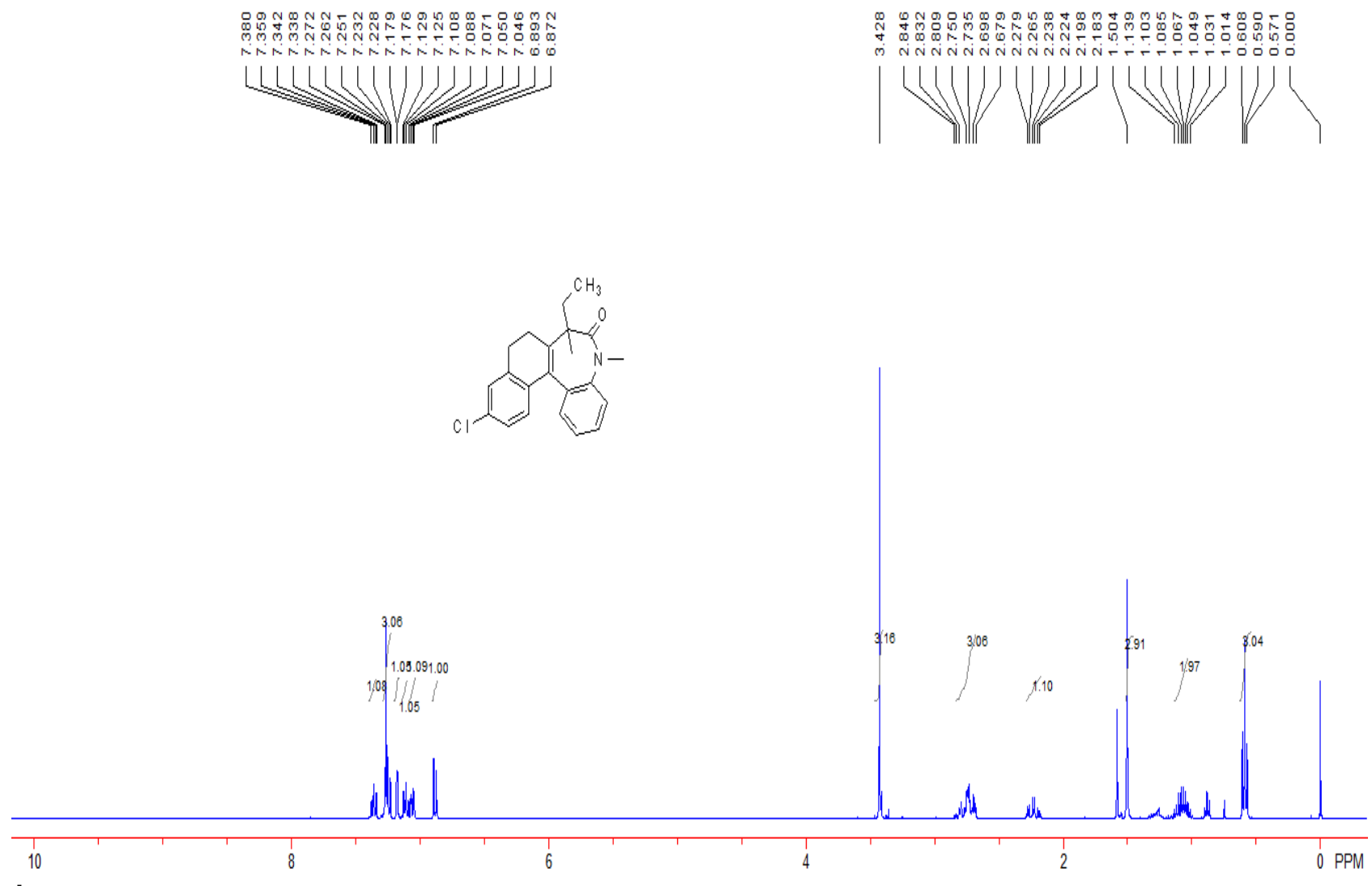

3e $\left({ }^{1} \mathrm{H}\right.$ NMR, $\left.400 \mathrm{MHz}, \mathrm{CDCl}_{3}\right)$
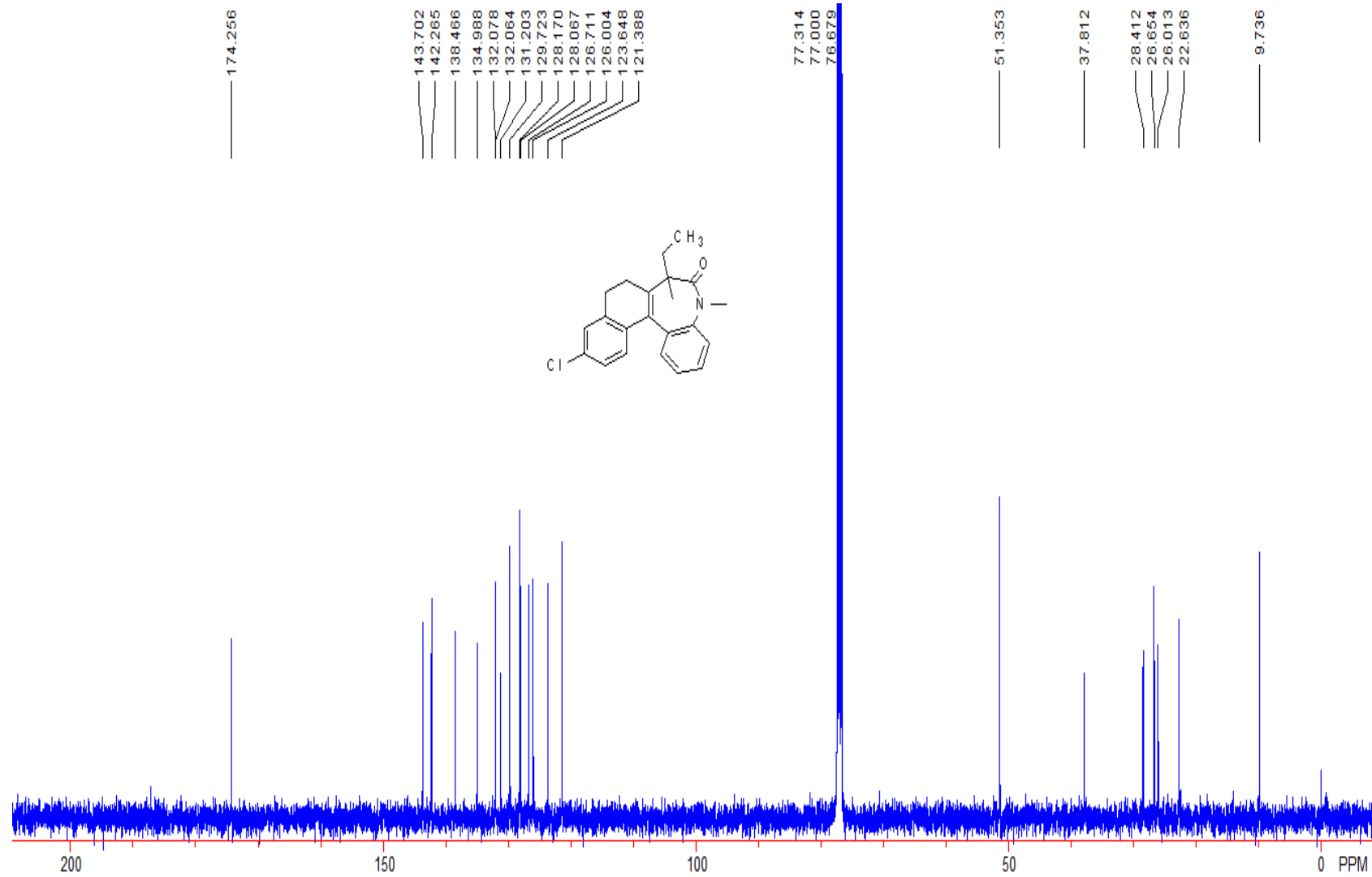

3e $\left({ }^{13} \mathrm{C}\right.$ NMR, $\left.100 \mathrm{MHz}, \mathrm{CDCl}_{3}\right)$ 


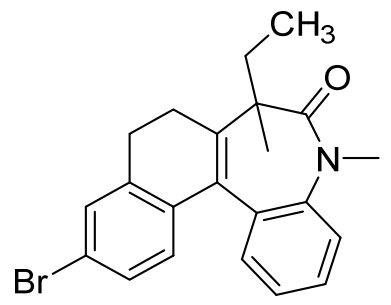

7-Ethyl-11-bromo-5,7-dimethyl-5,7,8,9-tetrahydro-6H-benzo[b]naphtho[1,2-d]azepin-6-one (3f)

Pale green oil, $64 \mathrm{mg}, 80 \%$ yield; Eluent: PE/EA $=10 / 1 .{ }^{1} \mathrm{H}$ NMR (400 $\left.\mathrm{MHz}, \mathrm{CDCl}_{3}, \mathrm{TMS}\right) \delta$ 7.38-7.33 (m, 2H), 7.27-7.20 (m, 3H), 7.13-7.09 (m, 1H), $6.82(\mathrm{~d}, J=8.4 \mathrm{~Hz}, 1 \mathrm{H}), 3.43(\mathrm{~s}, 3 \mathrm{H})$, 2.83-2.67 (m, 3H), 2.27-2.18 (m, 1H), $1.50(\mathrm{~s}, 3 \mathrm{H}), 1.12-1.03(\mathrm{~m}, 2 \mathrm{H}), 0.59(\mathrm{t}, J=7.2 \mathrm{~Hz}, 3 \mathrm{H}) ;{ }^{13} \mathrm{C}$ NMR (150 MHz, $\left.\mathrm{CDCl}_{3}, \mathrm{TMS}\right) \delta 174.2,143.9,142.3,138.8,135.5,132.0,131.3,129.7,129.6$, 129.0, 128.4, 128.2, 123.7, 121.4, 120.3, 51.4, 37.8, 28.3, 26.7, 26.1, 22.7, 9.8; IR $\left(\mathrm{CH}_{2} \mathrm{Cl}_{2}\right): v 2925$, 1644, 1596, 1494, 1445, 1384, 1355, 1243, 1168, 1095, 985, 876, 762, 726, 704, $678 \mathrm{~cm}^{-1}$; HRMS (ESI) m/z: (M+H) ${ }^{+}$Calcd. for $\mathrm{C}_{22} \mathrm{H}_{23} \mathrm{BrNO} 396.0958$; found 396.0960 . 


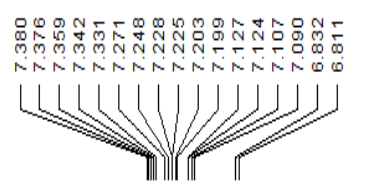

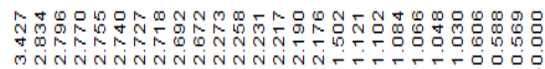
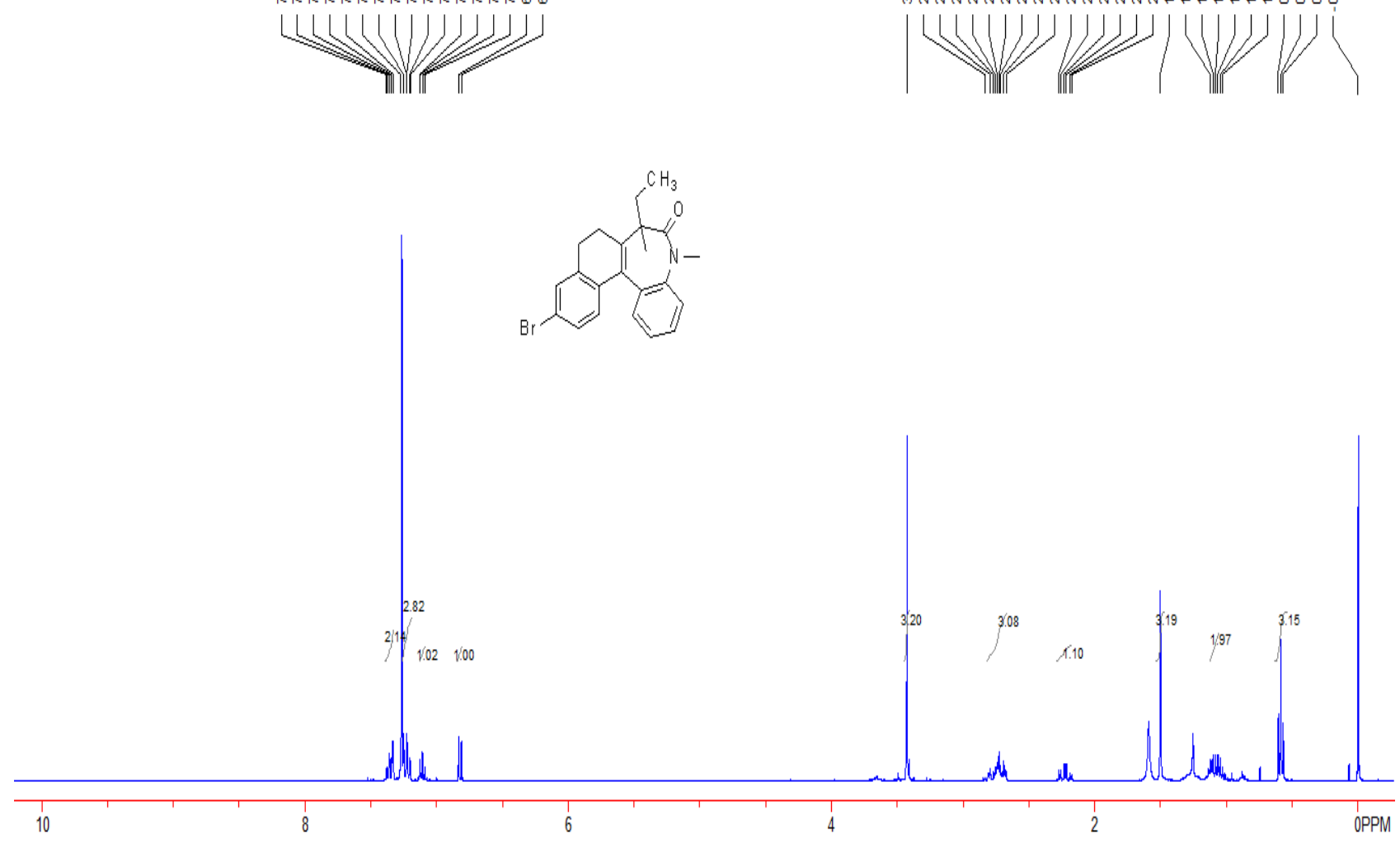

3f $\left({ }^{1} \mathrm{H} \mathrm{NMR}, 400 \mathrm{MHz}, \mathrm{CDCl}_{3}\right)$

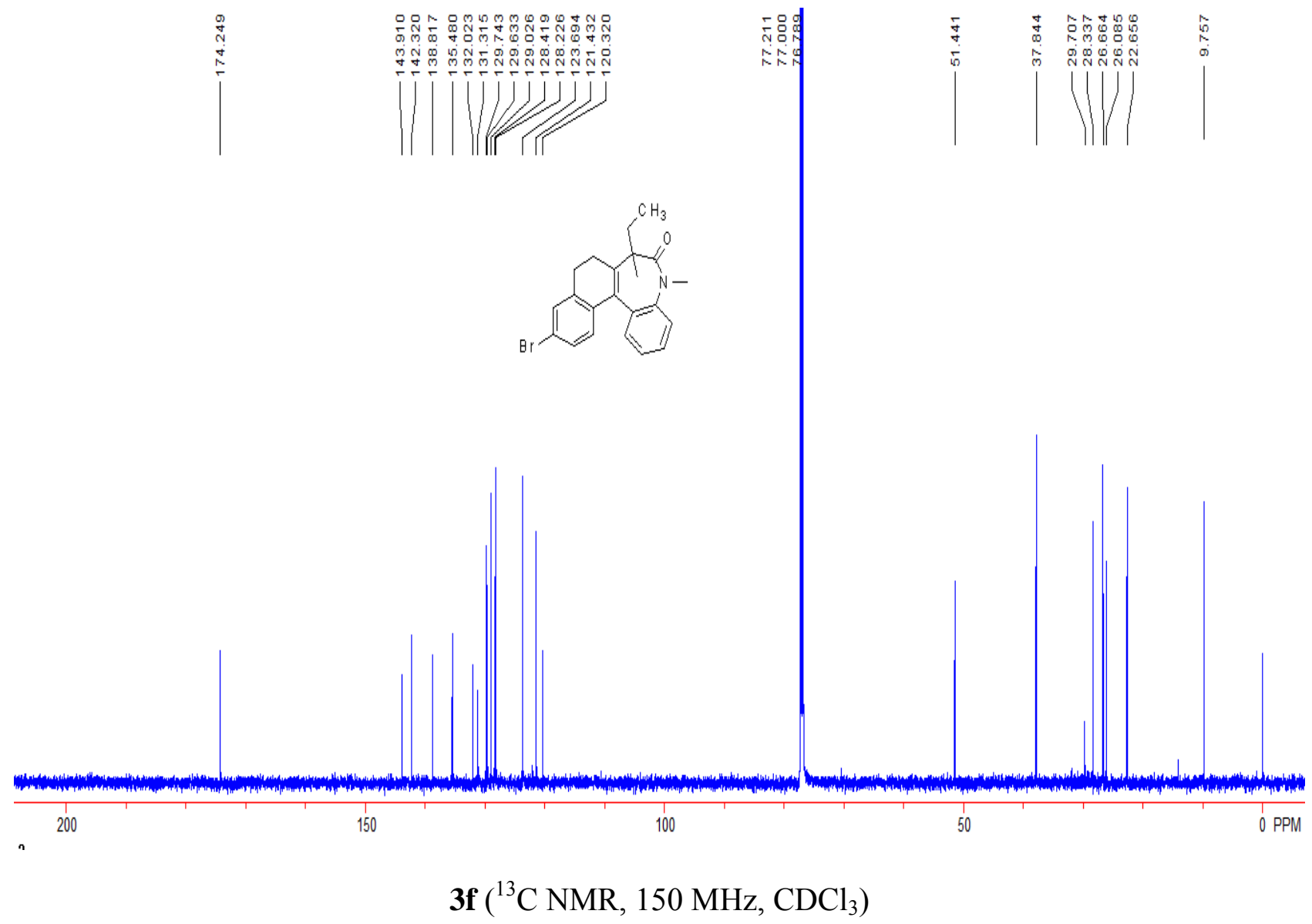




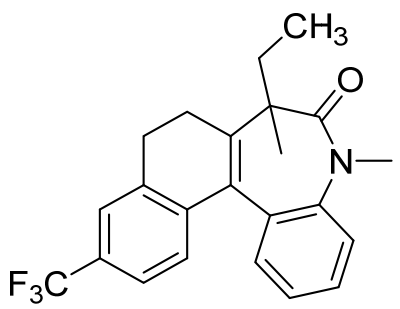

7-Ethyl-11-(trifluoromethyl)-5,7-dimethyl-5,7,8,9-tetrahydro-6H-benzo[b]naphtho[1,2-d]azepi n-6-one (3g)

Pale green oil, $61 \mathrm{mg}, 78 \%$ yield; Eluent: $\mathrm{PE} / \mathrm{EA}=10 / 1 .{ }^{1} \mathrm{H} \mathrm{NMR}\left(400 \mathrm{MHz}, \mathrm{CDCl}_{3}, \mathrm{TMS}\right) \delta 7.42$ (s, 1H), 7.40-7.22 (m, 5H), 7.14-7.11 (m, 1H), $7.06(\mathrm{~d}, J=8.0 \mathrm{~Hz}, 1 \mathrm{H}), 3.44(\mathrm{~s}, 3 \mathrm{H}), 2.87-2.75(\mathrm{~m}$, $3 \mathrm{H}), 2.32-2.24(\mathrm{~m}, 1 \mathrm{H}), 1.52(\mathrm{~s}, 3 \mathrm{H}), 1.15-1.06(\mathrm{~m}, 2 \mathrm{H}), 0.60(\mathrm{t}, J=7.2 \mathrm{~Hz}, 3 \mathrm{H}) ;{ }^{13} \mathrm{C} \mathrm{NMR}(100$ $\mathrm{MHz}, \mathrm{CDCl}_{3}$, TMS) $\delta 174.1,145.9,142.3,139.8,137.1,131.8,131.3,129.7,128.40(\mathrm{q}, J=32.1$ Hz), 128.37, 126.9, 124.2 (q, $J=270.6 \mathrm{~Hz}), 123.8,123.5(\mathrm{q}, J=3.7 \mathrm{~Hz}), 123.0(\mathrm{q}, J=3.7 \mathrm{~Hz})$, $121.5,51.5,37.9,28.3,26.6,26.0,22.6,9.8 ;{ }^{19} \mathrm{~F} \mathrm{NMR}\left(376 \mathrm{MHz}, \mathrm{CDCl}_{3}\right): \delta$-62.4; IR $\left(\mathrm{CH}_{2} \mathrm{Cl}_{2}\right): v$ 2917, 1648, 1495, 1355, 1118, 1099, 1074, 764, 669, $655 \mathrm{~cm}^{-1}$; HRMS (ESI) m/z: (M+H) Calcd. for $\mathrm{C}_{23} \mathrm{H}_{23} \mathrm{~F}_{3} \mathrm{NO} 386.1726$; found 386.1725. 

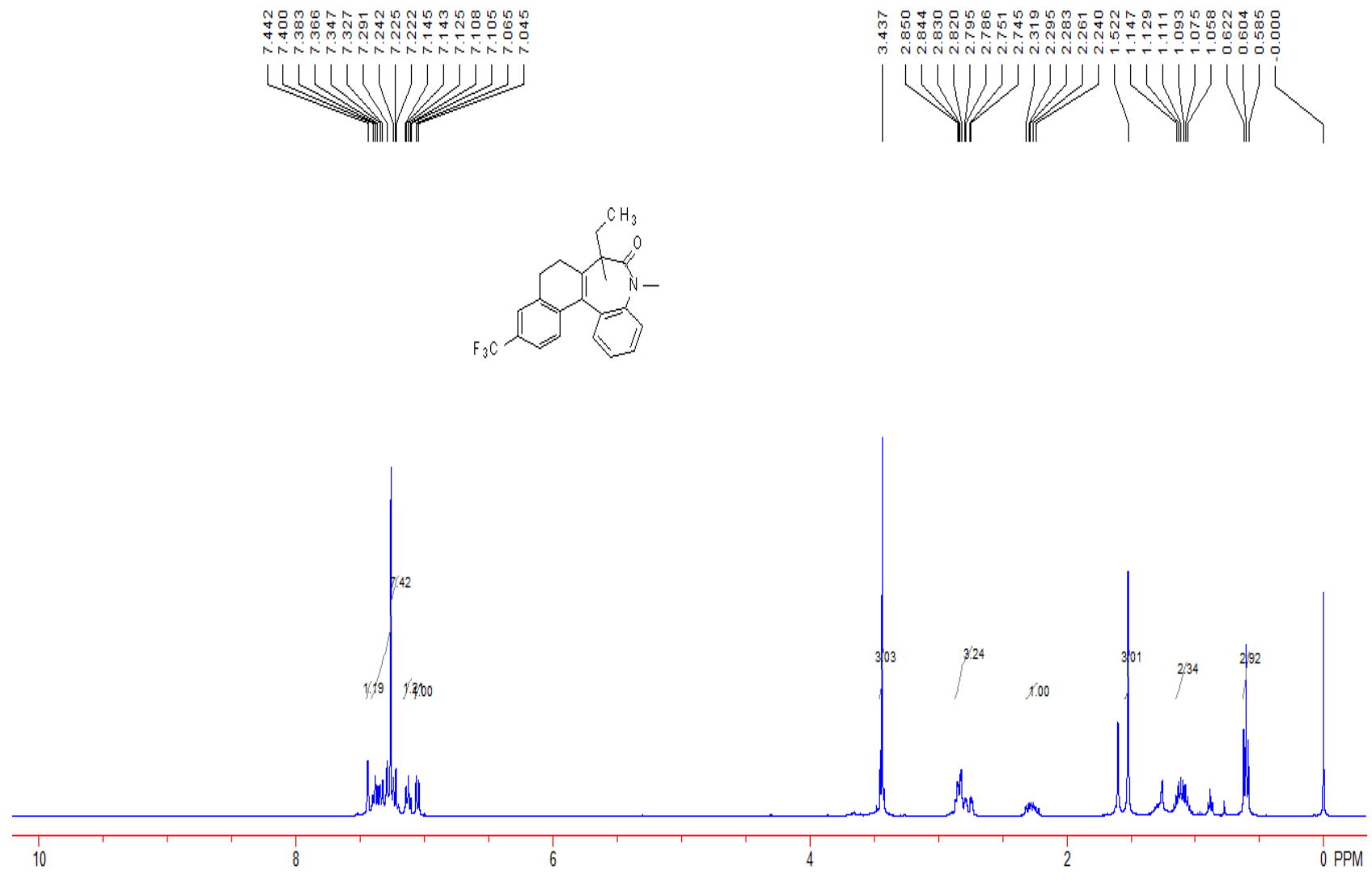

3g $\left({ }^{1} \mathrm{H}\right.$ NMR, $\left.400 \mathrm{MHz}, \mathrm{CDCl}_{3}\right)$
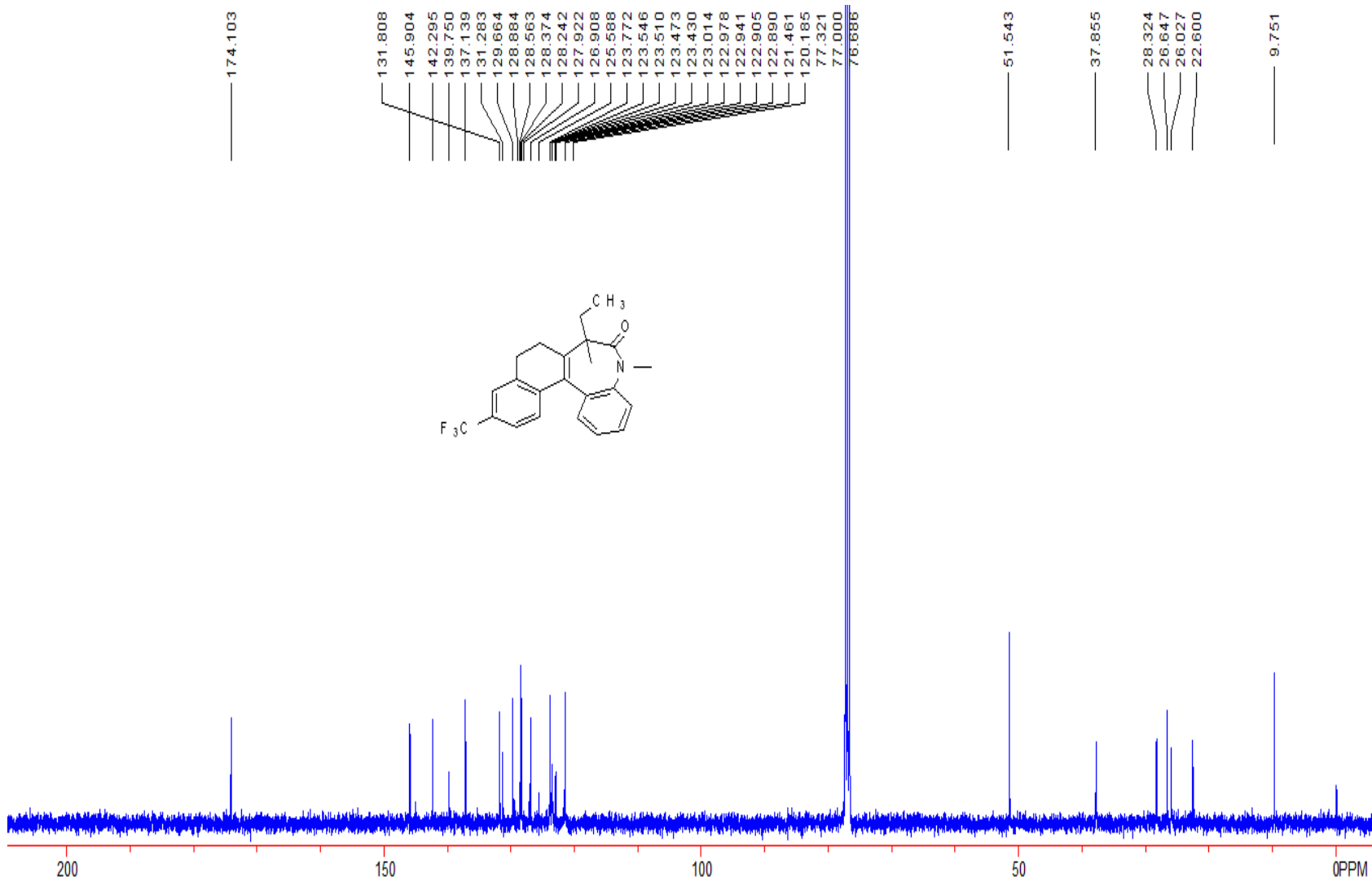

3g $\left({ }^{13} \mathrm{C} \mathrm{NMR}, 100 \mathrm{MHz}, \mathrm{CDCl}_{3}\right)$ 


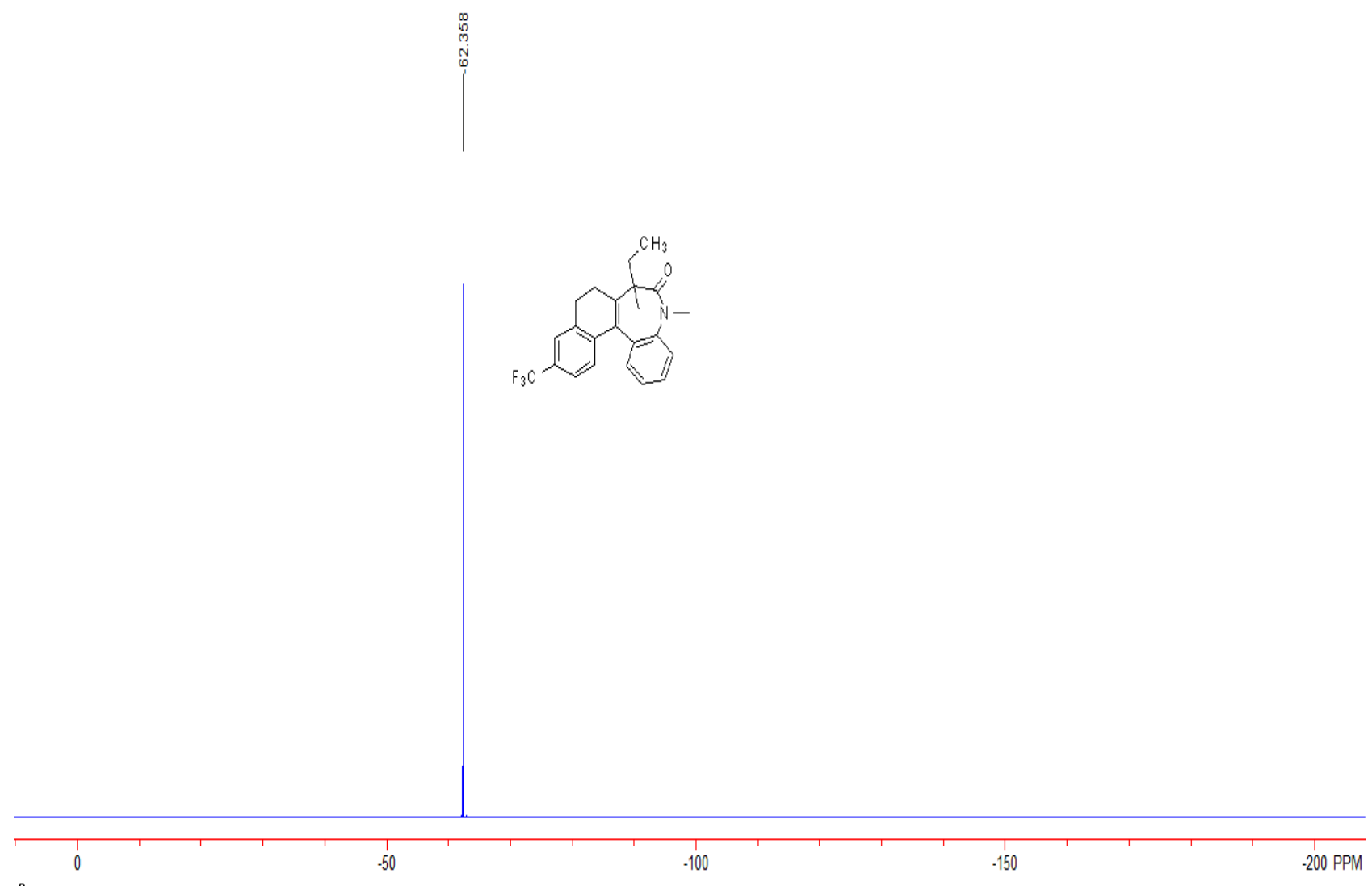

3g $\left({ }^{19} \mathrm{~F} \mathrm{NMR,} 376 \mathrm{MHz}, \mathrm{CDCl}_{3}\right)$ 


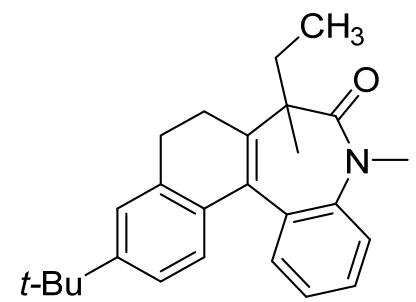

7-Ethyl-11-(tert-butyl)-5,7-dimethyl-5,7,8,9-tetrahydro-6H-benzo[b]naphtho[1,2-d]azepin-6-o ne (3h)

Pale green oil, $65 \mathrm{mg}, 87 \%$ yield; Eluent: $\mathrm{PE} / \mathrm{EA}=10 / 1 .{ }^{1} \mathrm{H}$ NMR $\left(400 \mathrm{MHz}, \mathrm{CDCl}_{3}, \mathrm{TMS}\right) \delta$ 7.34-7.33 (m, 2H), 7.24-7.21 (m, 2H), 7.13-7.09 (m, 2H), $6.90(\mathrm{~d}, J=8.0 \mathrm{~Hz}, 1 \mathrm{H}), 3.40(\mathrm{~s}, 3 \mathrm{H})$, 2.84-2.69 (m, 3H), 2.30-2.21 (m, 1H), $1.50(\mathrm{~s}, 3 \mathrm{H}), 1.32(\mathrm{~s}, 9 \mathrm{H}), 1.13-1.05(\mathrm{~m}, 2 \mathrm{H}), 0.59(\mathrm{t}, J=7.2$ $\mathrm{Hz}, 3 \mathrm{H}) ;{ }^{13} \mathrm{C} \mathrm{NMR}\left(100 \mathrm{MHz}, \mathrm{CDCl}_{3}\right.$, TMS) $\delta 174.4,149.8,142.6,142.3,136.1,133.8,132.5$, 131.6, 130.0, 127.8, 126.4, 123.9, 123.4, 122.8, 121.3, 51.3, 37.8, 34.5, 31.3, 29.0, 26.7, 26.4, 22.7, 9.8; IR $\left(\mathrm{CH}_{2} \mathrm{Cl}_{2}\right):$ v 2988, 1645, 1496, 1447, 1379, 1255, 1089, 1051, 766, 719, $668 \mathrm{~cm}^{-1}$; HRMS (ESI) $\mathrm{m} / \mathrm{z}$ : $(\mathrm{M}+\mathrm{H})^{+}$Calcd. for $\mathrm{C}_{26} \mathrm{H}_{32} \mathrm{NO} 374.2478$; found 374.2479 . 


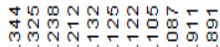

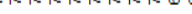

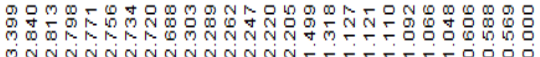

(1) II)

(4)

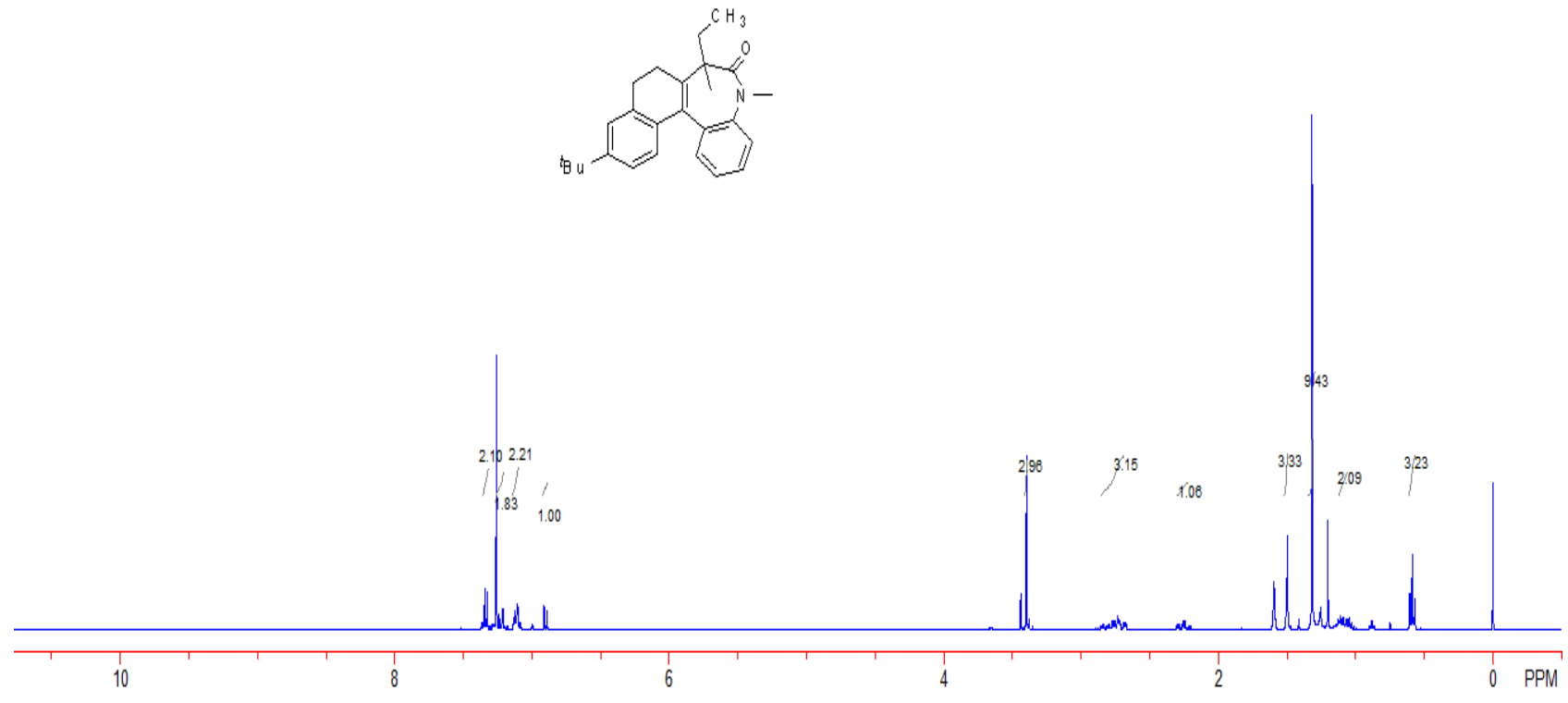

WUW

3h $\left({ }^{1} \mathrm{H}\right.$ NMR, $\left.400 \mathrm{MHz}, \mathrm{CDCl}_{3}\right)$
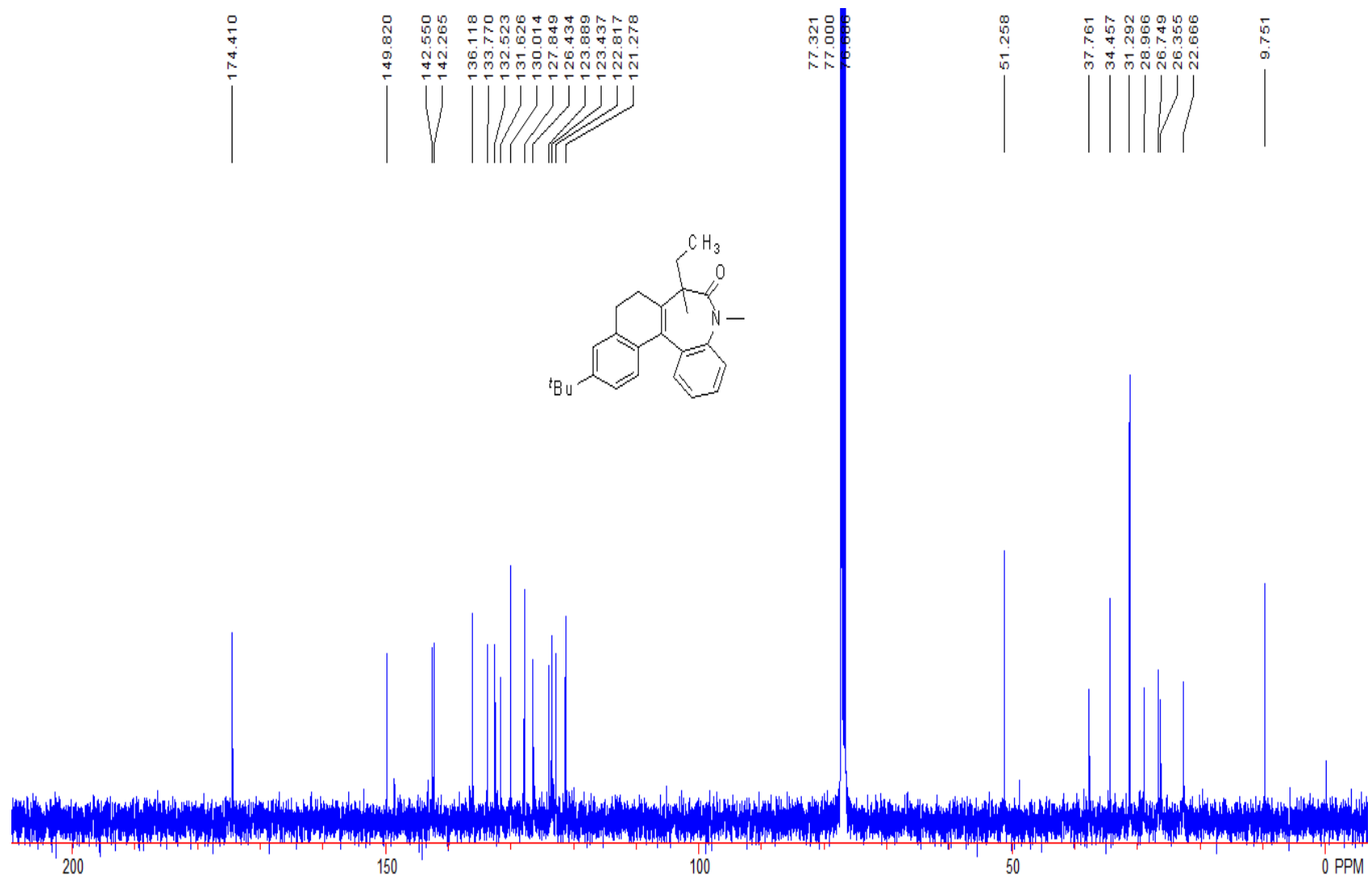

3h $\left({ }^{13} \mathrm{C} \mathrm{NMR}, 100 \mathrm{MHz}, \mathrm{CDCl}_{3}\right)$ 


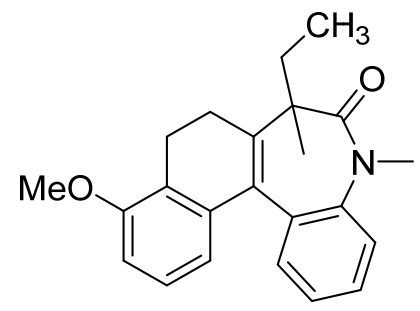

7-Ethyl-10-methoxy-5,7-dimethyl-5,7,8,9-tetrahydro-6H-benzo[b]naphtho[1,2-d]azepin-6-one (3i)

Pale green oil, $57 \mathrm{mg}, 82 \%$ yield; Eluent: $\mathrm{PE} / \mathrm{EA}=10 / 1 .{ }^{1} \mathrm{H}$ NMR $\left(400 \mathrm{MHz}, \mathrm{CDCl}_{3}, \mathrm{TMS}\right) \delta$ 7.36-7.32 (m, 1H), 7.26-7.24 (m, 2H), 7.11-7.04 (m, 2H), $6.78(\mathrm{~d}, J=8.4 \mathrm{~Hz}, 1 \mathrm{H}), 6.58(\mathrm{~d}, J=7.6$ $\mathrm{Hz}, 1 \mathrm{H}), 3.87(\mathrm{~s}, 3 \mathrm{H}), 3.42(\mathrm{~s}, 3 \mathrm{H}), 3.25-3.20(\mathrm{~m}, 1 \mathrm{H}), 2.73-2.67(\mathrm{~m}, 1 \mathrm{H}), 2.36\left(\mathrm{td}, J_{1}=5.6 \mathrm{~Hz}, J_{2}=\right.$ $16.4 \mathrm{~Hz}, 1 \mathrm{H}), 2.15\left(\mathrm{td}, J_{1}=5.6 \mathrm{~Hz}, J_{2}=16.8 \mathrm{~Hz}, 1 \mathrm{H}\right), 1.51(\mathrm{~s}, 3 \mathrm{H}), 1.12-1.05(\mathrm{~m}, 2 \mathrm{H}), 0.59(\mathrm{t}, J=$ $7.6 \mathrm{~Hz}, 3 \mathrm{H}) ;{ }^{13} \mathrm{C} \mathrm{NMR}\left(100 \mathrm{MHz}, \mathrm{CDCl}_{3}\right.$, TMS) $\delta 174.5,155.5,143.8,142.2,137.7,132.7,131.8$, $130.1,127.8,126.0,124.8,123.4,121.2,119.8,109.2$, 55.6, 51.3, 37.8, 26.7, 25.7, 22.6, 20.3, 9.8; IR $\left(\mathrm{CH}_{2} \mathrm{Cl}_{2}\right): v 2929,1637,1596,1466,1445,1358,1264,1081,1069,786,750,704,669 \mathrm{~cm}^{-1}$; HRMS (ESI) m/z: $(\mathrm{M}+\mathrm{H})^{+}$Calcd. for $\mathrm{C}_{23} \mathrm{H}_{26} \mathrm{NO}_{2}$ 348.1958, found 348.1953. 


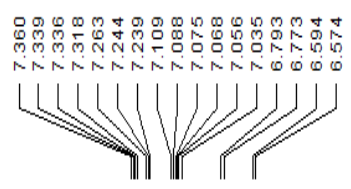

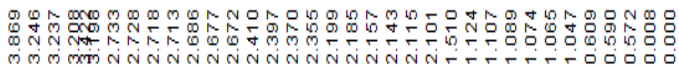
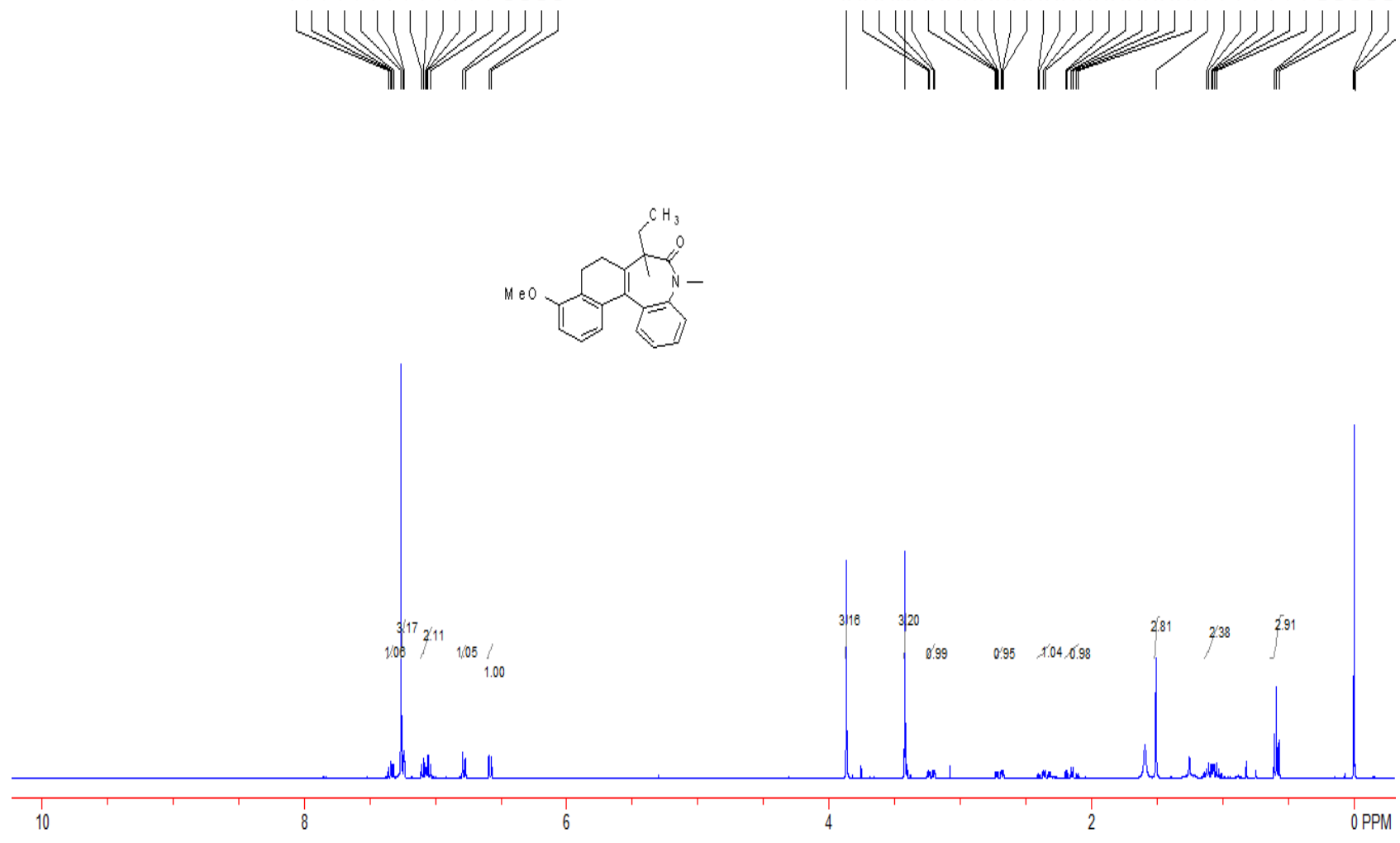

3i ( ${ }^{1} \mathrm{H}$ NMR, $\left.400 \mathrm{MHz}, \mathrm{CDCl}_{3}\right)$
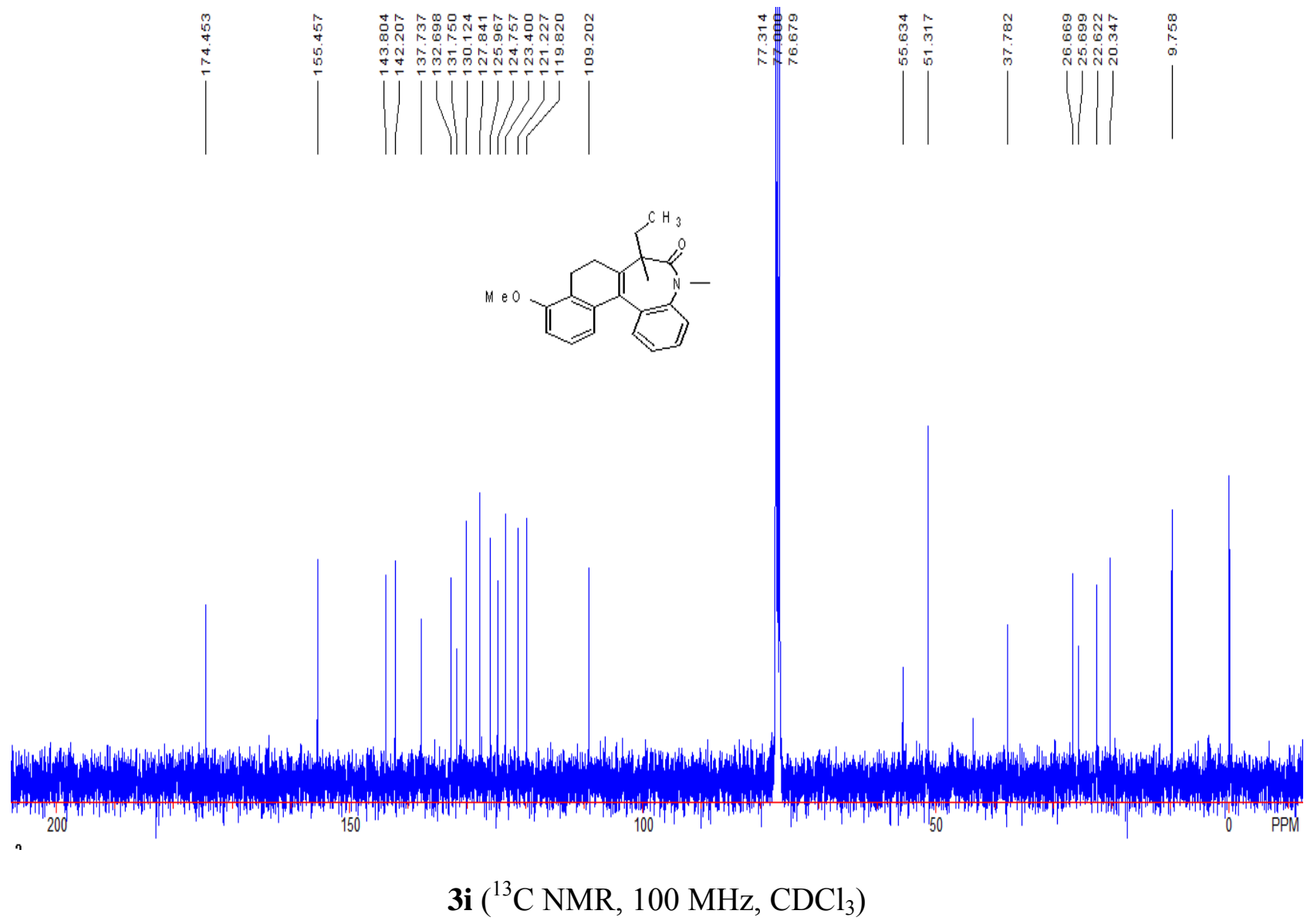


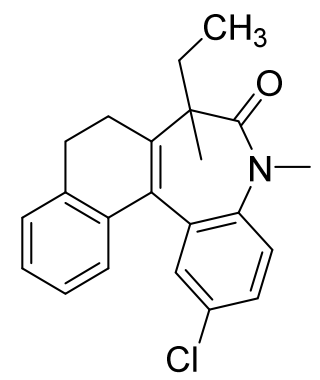

2-Chloro-7-ethyl-5,7-dimethyl-5,7,8,9-tetrahydro-6H-benzo[b]naphtho[1,2-d]azepin-6-one (3k) Pale green oil, $51 \mathrm{mg}, 73 \%$ yield; Eluent: PE/EA = 10/1. ${ }^{1} \mathrm{H}$ NMR (400 $\left.\mathrm{MHz}, \mathrm{CDCl}_{3}, \mathrm{TMS}\right) \delta$ 7.33-7.29 (m, 2H), 7.21-7.12 (m, 4H), $6.95(\mathrm{~d}, J=7.2 \mathrm{~Hz}, 1 \mathrm{H}), 3.40(\mathrm{~s}, 3 \mathrm{H}), 2.82-2.69(\mathrm{~m}, 3 \mathrm{H})$, $2.24\left(\mathrm{td}, J_{1}=6.4 \mathrm{~Hz}, J_{2}=16.4 \mathrm{~Hz}, 1 \mathrm{H}\right), 1.51(\mathrm{~s}, 3 \mathrm{H}), 1.19-1.07(\mathrm{~m}, 2 \mathrm{H}), 0.62(\mathrm{t}, J=7.2 \mathrm{~Hz}, 3 \mathrm{H})$; ${ }^{13} \mathrm{C}$ NMR $\left(150 \mathrm{MHz}, \mathrm{CDCl}_{3}, \mathrm{TMS}\right) \delta 174.1,144.7,140.9,136.6,136.0,134.1,131.1,129.4,128.9$, 128.1, 127.1, 126.9, 126.6, 126.3, 122.7, 51.5, 37.8, 28.5, 26.9, 26.3, 22.6, 9.8; IR $\left(\mathrm{CH}_{2} \mathrm{Cl}_{2}\right): v 2931$, 2922, 1637, 1485, 1445, 1106, 1091, 829, 770, 711, $657 \mathrm{~cm}^{-1}$; HRMS (ESI) m/z: (M+H) Calcd. for $\mathrm{C}_{22} \mathrm{H}_{23} \mathrm{ClNO} 352.1463$; found 352.1461. 


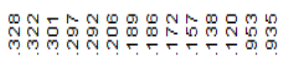

L...W

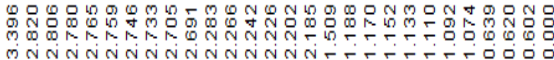

מul<smiles>CCC1(C)C(=O)N(C)c2ccc(Cl)cc2C1=C1CCc2ccccc21</smiles>

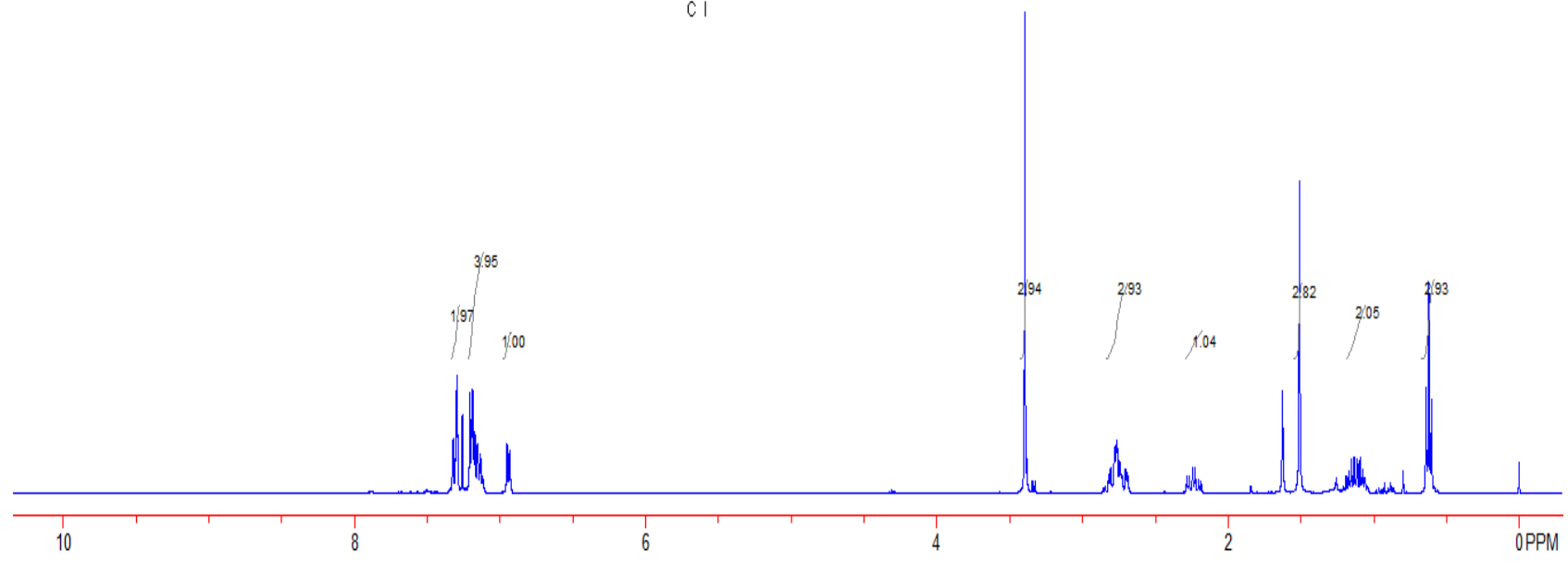

3k $\left({ }^{1} \mathrm{H}\right.$ NMR, $\left.400 \mathrm{MHz}, \mathrm{CDCl}_{3}\right)$
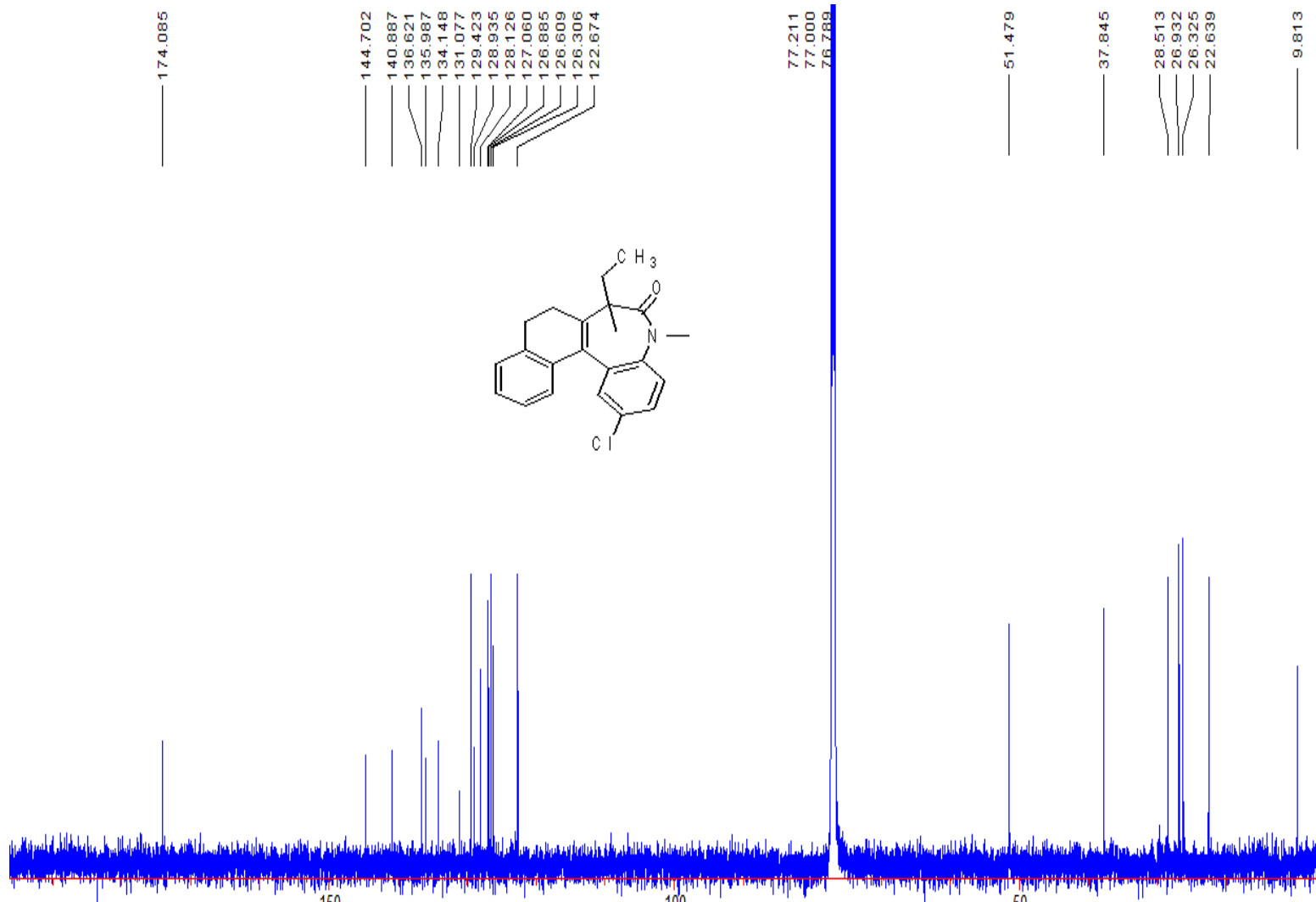


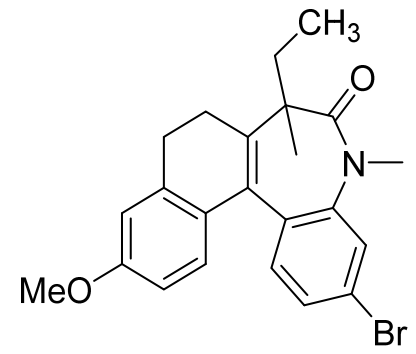

3-Bromo-7-ethyl-11-methoxy-5,7-dimethyl-5,7,8,9-tetrahydro-6H-benzo[b]naphtho[1,2-d]azepi n-6-one (3l)

Pale green oil, $64 \mathrm{mg}, 75 \%$ yield; Eluent: $\mathrm{PE} / \mathrm{EA}=10 / 1 .{ }^{1} \mathrm{H} \mathrm{NMR}\left(400 \mathrm{MHz}, \mathrm{CDCl}_{3}, \mathrm{TMS}\right) \delta 7.40$ $(\mathrm{d}, J=2.0 \mathrm{~Hz}, 1 \mathrm{H}), 7.24-7.16(\mathrm{~m}, 2 \mathrm{H}), 6.83(\mathrm{~d}, J=8.4 \mathrm{~Hz}, 1 \mathrm{H}), 6.75(\mathrm{~d}, J=2.4 \mathrm{~Hz}, 1 \mathrm{H}), 6.63$ (dd, $\left.J_{1}=2.8 \mathrm{~Hz}, J_{2}=8.4 \mathrm{~Hz}, 1 \mathrm{H}\right), 3.81(\mathrm{~s}, 3 \mathrm{H}), 3.41(\mathrm{~s}, 3 \mathrm{H}), 2.84-2.66(\mathrm{~m}, 3 \mathrm{H}), 2.21\left(\mathrm{td}, J_{1}=5.6 \mathrm{~Hz}, J_{2}\right.$ $=16.4 \mathrm{~Hz}, 1 \mathrm{H}), 1.50(\mathrm{~s}, 3 \mathrm{H}), 1.18-1.05(\mathrm{~m}, 2 \mathrm{H}), 0.61(\mathrm{t}, J=7.2 \mathrm{~Hz}, 3 \mathrm{H}) ;{ }^{13} \mathrm{C} \mathrm{NMR}(150 \mathrm{MHz}$, $\mathrm{CDCl}_{3}$, TMS) $\delta 174.3,158.6,143.4,141.3,138.5,131.6,131.4,130.9,129.1,127.9,126.6,124.1$, 121.0, 112.7, 111.0, 55.3, 51.3, 37.8, 29.0, 26.7, 26.2, 22.7, 9.7; IR $\left(\mathrm{CH}_{2} \mathrm{Cl}_{2}\right): v 2959,2919,1649$, 1602, 1501, 1462, 1244, 1176, 829, 818, 706, $669 \mathrm{~cm}^{-1}$; HRMS (ESI) m/z: $(\mathrm{M}+\mathrm{H})^{+}$Calcd. for $\mathrm{C}_{23} \mathrm{H}_{25} \mathrm{BrNO}_{2}$ 426.1063; found 426.1052 . 


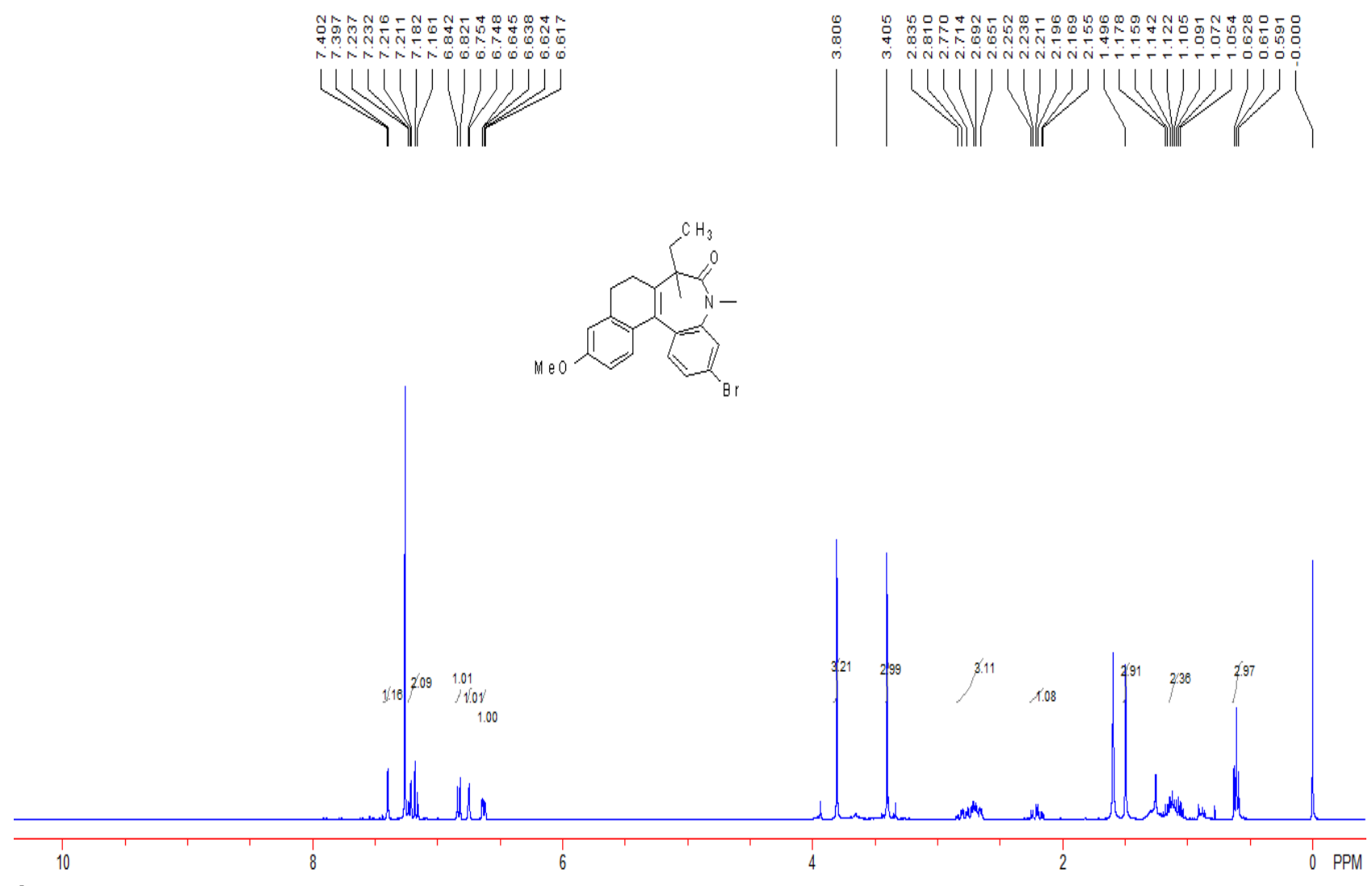

$\left.3 \mathbf{~ ( ~}{ }^{1} \mathrm{H} \mathrm{NMR}, 400 \mathrm{MHz}, \mathrm{CDCl}_{3}\right)$
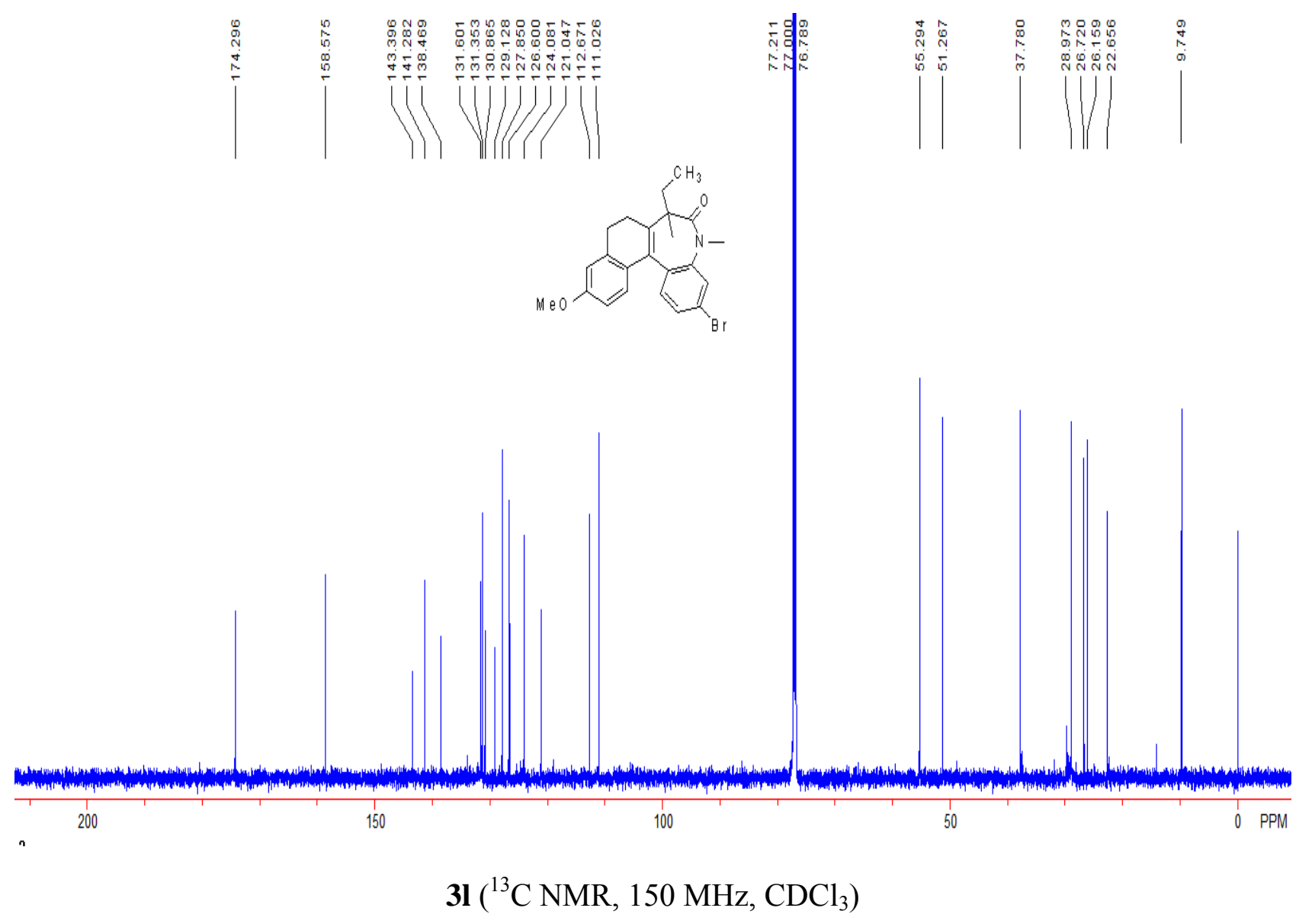


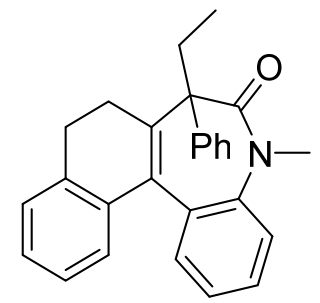

7-Ethyl-5-methyl-7-phenyl-5,7,8,9-tetrahydro-6H-benzo[b]naphtho[1,2-d]azepin-6-one (3m)

Pale green oil, $60 \mathrm{mg}$, 79\% yield; Eluent: PE/EA = 10/1. ${ }^{1} \mathrm{H}$ NMR (400 $\left.\mathrm{MHz}, \mathrm{CDCl}_{3}, \mathrm{TMS}\right) \delta$ 7.26-7.25 (m, 1H), 7.19-7.15 (m, 1H), $7.08(\mathrm{t}, J=7.2 \mathrm{~Hz}, 1 \mathrm{H}), 6.99-6.97(\mathrm{~m}, 1 \mathrm{H}), 6.96-6.81(\mathrm{~m}$, 8H), 6.75-6.71 (m, 1H), $3.45(\mathrm{~s}, 3 \mathrm{H}), 3.13-3.04(\mathrm{~m}, 1 \mathrm{H}), 2.94-2.84(\mathrm{~m}, 2 \mathrm{H}), 2.40\left(\mathrm{td}, J_{1}=5.2 \mathrm{~Hz}, J_{2}\right.$ $=16.8 \mathrm{~Hz}, 1 \mathrm{H}), 2.29-2.18(\mathrm{~m}, 2 \mathrm{H}), 0.61(\mathrm{t}, J=7.2 \mathrm{~Hz}, 3 \mathrm{H}) ;{ }^{13} \mathrm{C} \mathrm{NMR}\left(150 \mathrm{MHz}, \mathrm{CDCl}_{3}, \mathrm{TMS}\right) \delta$ $172.3,142.6,142.1,139.8,136.7,136.0,133.9$, 133.1, 130.0, 127.6, 127.1, 127.01, 126.99, 126.8, 126.1, 125.6, 123.0, 122.0, 59.9, 37.2, 31.3, 28.6, 25.2, 10.2; IR $\left(\mathrm{CH}_{2} \mathrm{Cl}_{2}\right): v 2923,1644,1596$, $1580,1492,1446,1351,1256,1241,1091,983,875,820,762,736,724,704,675 \mathrm{~cm}^{-1}$; HRMS (ESI) $\mathrm{m} / \mathrm{z}$ : $(\mathrm{M}+\mathrm{H})^{+}$Calcd. for $\mathrm{C}_{27} \mathrm{H}_{26} \mathrm{NO} 380.2009$; found 380.2004 . 


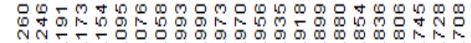

ARTRNR

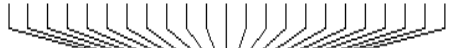

(a)

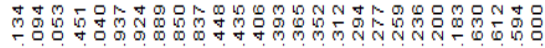

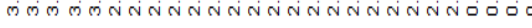

Ly MU U U

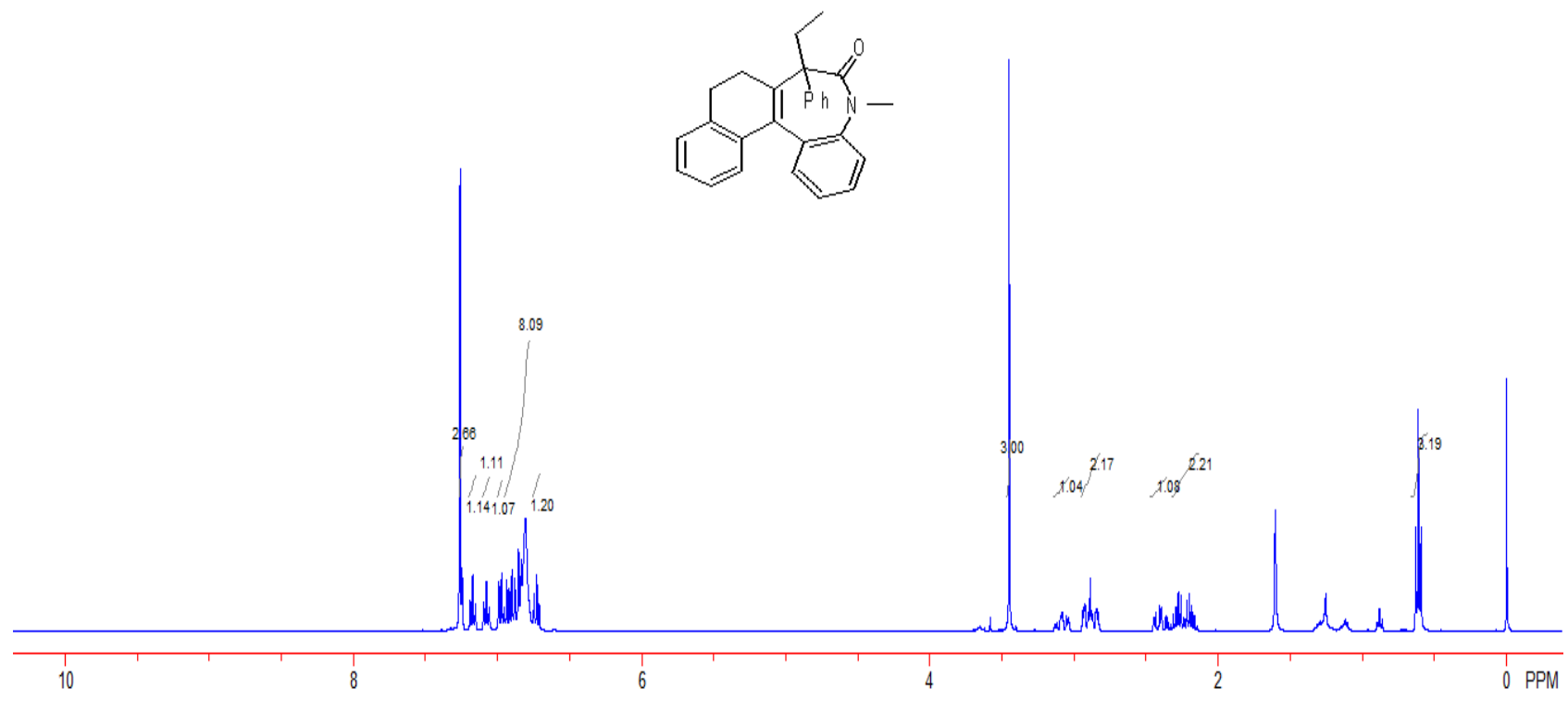

3m $\left({ }^{1} \mathrm{H}\right.$ NMR, $\left.400 \mathrm{MHz}, \mathrm{CDCl}_{3}\right)$
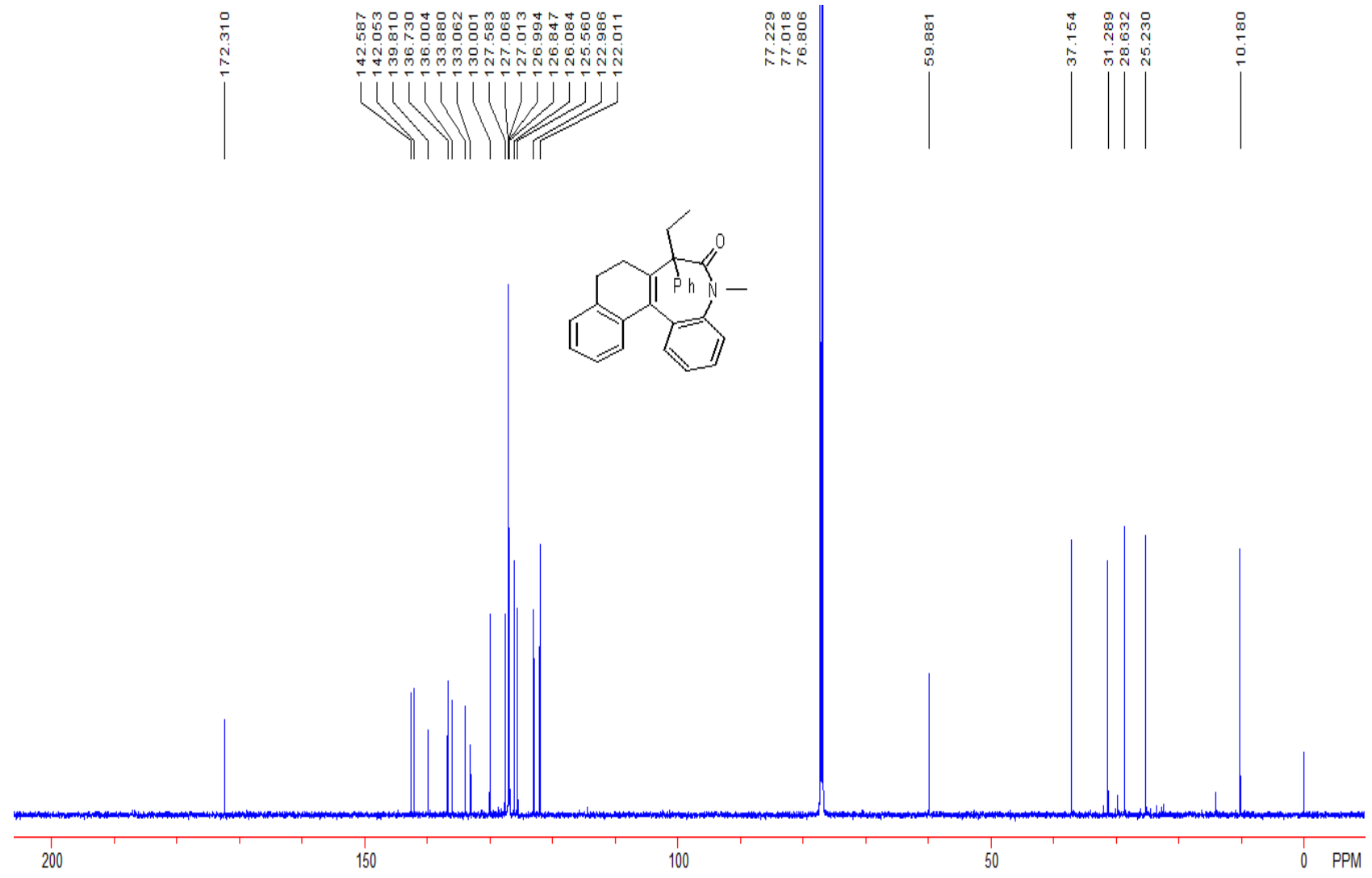

3m $\left({ }^{13} \mathrm{C} \mathrm{NMR,} 150 \mathrm{MHz}, \mathrm{CDCl}_{3}\right)$ 


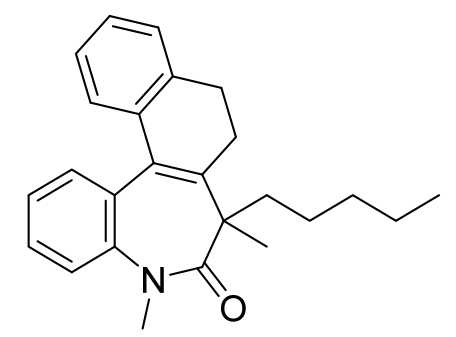

\section{5,7-Dimethyl-7-pentyl-5,7,8,9-tetrahydro-6H-benzo[b]naphtho[1,2-d]azepin-6-one (3p)}

Pale green oil, $55 \mathrm{mg}, 76 \%$ yield; Eluent: PE/EA $=10 / 1 .{ }^{1} \mathrm{H}$ NMR (400 $\left.\mathrm{MHz}, \mathrm{CDCl}_{3}, \mathrm{TMS}\right) \delta$ 7.37-7.33 (m, 1H), 7.30-7.24 (m, 2H), 7.20-7.16 (m, 1H), 7.15-7.09 (m, 3H), $6.96(\mathrm{~d}, J=7.6 \mathrm{~Hz}$, $1 \mathrm{H}), 3.41(\mathrm{~s}, 3 \mathrm{H}), 2.83-2.68(\mathrm{~m}, 3 \mathrm{H}), 2.25\left(\mathrm{td}, J_{1}=6.0 \mathrm{~Hz}, J_{2}=16.4 \mathrm{~Hz}, 1 \mathrm{H}\right), 1.52(\mathrm{~s}, 3 \mathrm{H})$, 1.13-0.93 (m, 6H), 0.83-0.76 (m, 2H), $0.71(\mathrm{t}, J=7.2 \mathrm{~Hz}, 3 \mathrm{H}) ;{ }^{13} \mathrm{C} \mathrm{NMR}\left(150 \mathrm{MHz}, \mathrm{CDCl}_{3}, \mathrm{TMS}\right)$ $\delta 174.5,143.8,142.3,136.6,136.5,132.6,131.8,130.0,127.9,126.8,126.72,126.70,126.0,123.5$, 121.4, 50.9, 37.7, 34.1, 31.9, 28.7, 26.1, 25.1, 23.3, 22.1, 13.9; IR $\left(\mathrm{CH}_{2} \mathrm{Cl}_{2}\right): v 2956,2919,2900$, 1644, 1605, 1469, 1377, 1081, 967, 815, 771, $669 \mathrm{~cm}^{-1}$; HRMS (ESI) m/z: $(\mathrm{M}+\mathrm{H})^{+}$Calcd. for $\mathrm{C}_{25} \mathrm{H}_{30} \mathrm{NO} 360.2322$; found 360.2321 . 


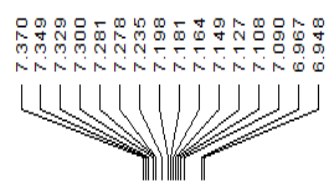

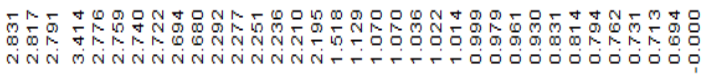

LIU W W W
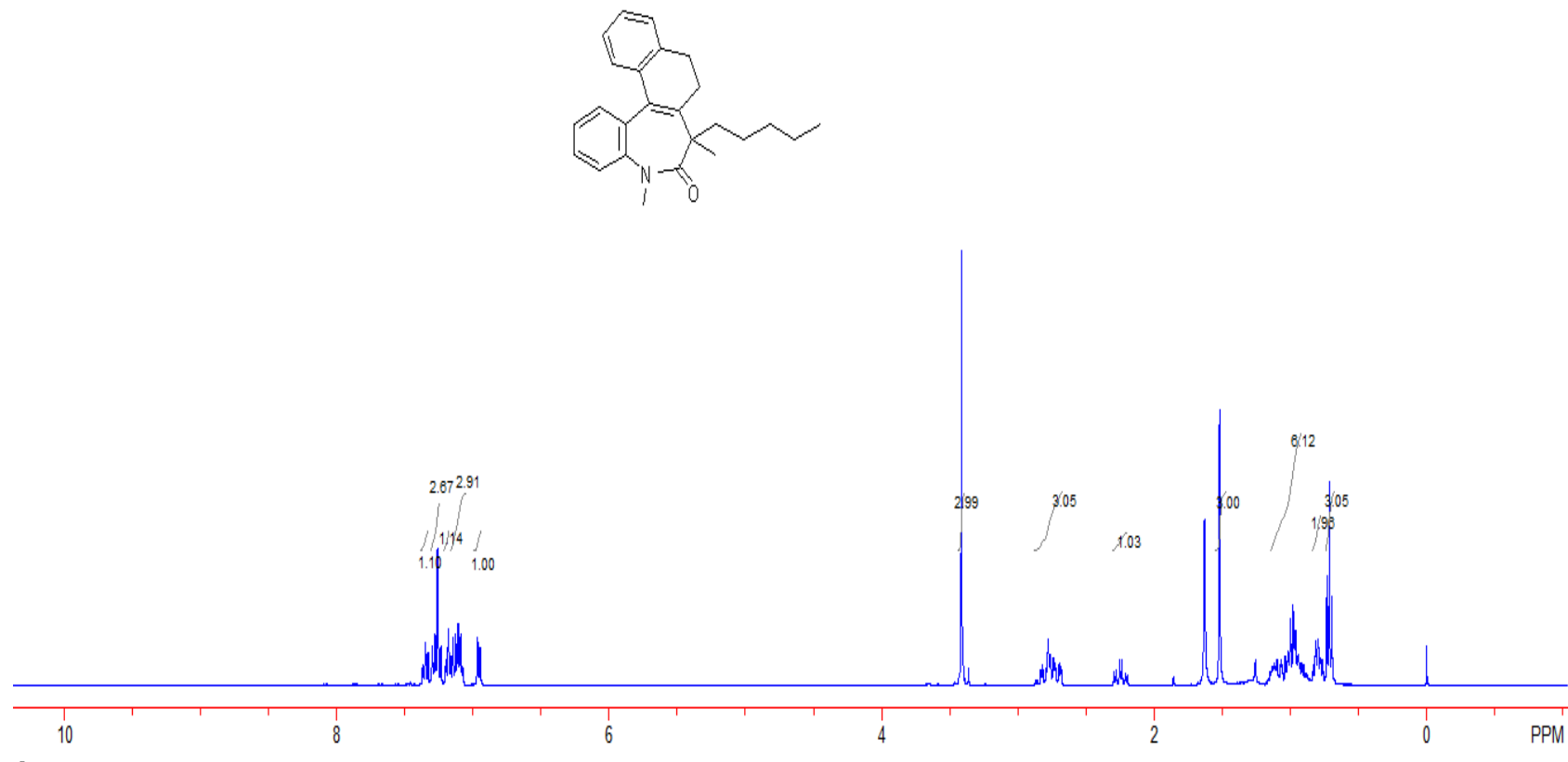

3p $\left({ }^{1} \mathrm{H}\right.$ NMR, $\left.400 \mathrm{MHz}, \mathrm{CDCl}_{3}\right)$
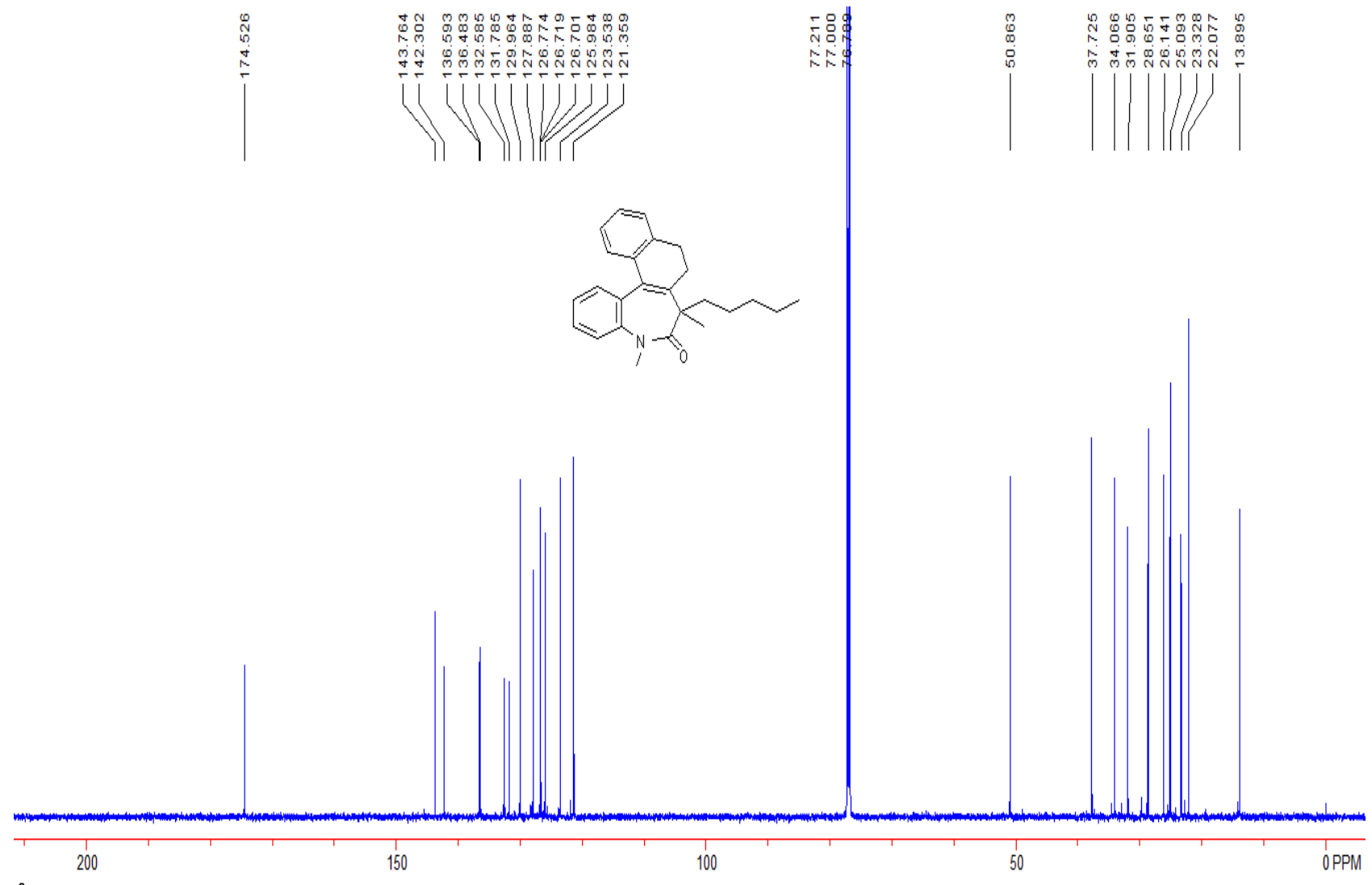

3p $\left({ }^{13} \mathrm{C}\right.$ NMR, $\left.150 \mathrm{MHz}, \mathrm{CDCl}_{3}\right)$ 


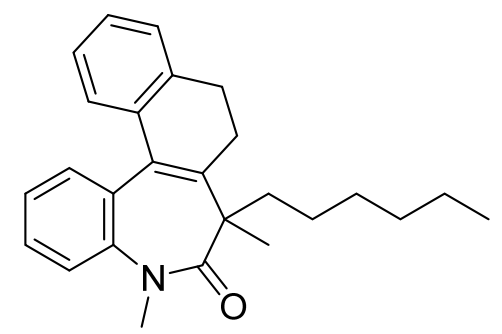

5,7-Dimethyl-7-hexyl-5,7,8,9-tetrahydro-6H-benzo[b]naphtho[1,2-d]azepin-6-one (3q)

Pale green oil, $59 \mathrm{mg}, 79 \%$ yield; Eluent: PE/EA = 10/1. ${ }^{1} \mathrm{H}$ NMR (400 $\left.\mathrm{MHz}, \mathrm{CDCl}_{3}, \mathrm{TMS}\right) \delta$ 7.37-7.33 (m, 1H), 7.30-7.24 (m, 2H), 7.20-7.17 (m, 1H), 7.15-7.08 (m, 3H), 6.96 (d, J = 7.2 Hz, $1 \mathrm{H}), 3.41(\mathrm{~s}, 3 \mathrm{H}), 2.83-2.68(\mathrm{~m}, 3 \mathrm{H}), 2.25\left(\mathrm{td}, J_{1}=6.4 \mathrm{~Hz}, J_{2}=16.4 \mathrm{~Hz}, 1 \mathrm{H}\right), 1.52(\mathrm{~s}, 3 \mathrm{H})$, 1.13-1.04 (m, 4H), 0.97-0.90 (m, 4H), 0.85-0.75 (m, 5H); $\left.{ }^{13} \mathrm{C} \mathrm{NMR} \mathrm{(150} \mathrm{MHz,} \mathrm{CDCl}_{3}, \mathrm{TMS}\right) \delta$ $174.5,143.8,142.3,136.6,136.5,132.6,131.8,130.0,127.9,126.8,126.73,126.70,126.0,123.6$, 121.4, 50.9, 37.7, 34.1, 31.3, 29.3, 28.7, 26.2, 25.4, 23.3, 22.4, 13.9; IR $\left(\mathrm{CH}_{2} \mathrm{Cl}_{2}\right): v 2989,2941$, 2901, 1647, 1597, 1494, 1379, 1355, 1256, 1067, 1050, 764, 719, 699, $668 \mathrm{~cm}^{-1}$; HRMS (ESI) m/z: $(\mathrm{M}+\mathrm{H})^{+}$Calcd. for $\mathrm{C}_{26} \mathrm{H}_{32} \mathrm{NO} 374.2478$; found 374.2472. 

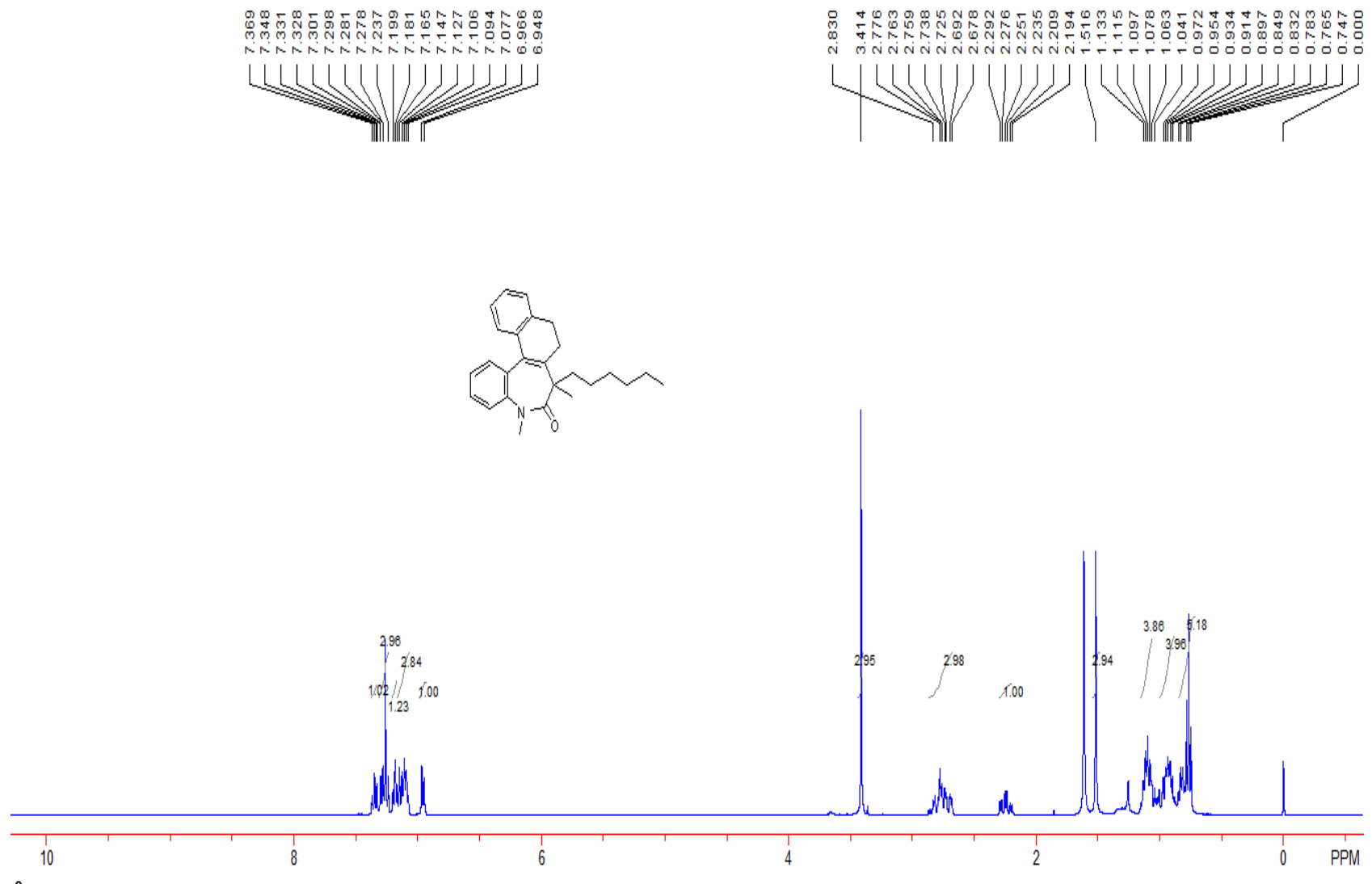

3q $\left({ }^{1} \mathrm{H} \mathrm{NMR}, 400 \mathrm{MHz}, \mathrm{CDCl}_{3}\right)$
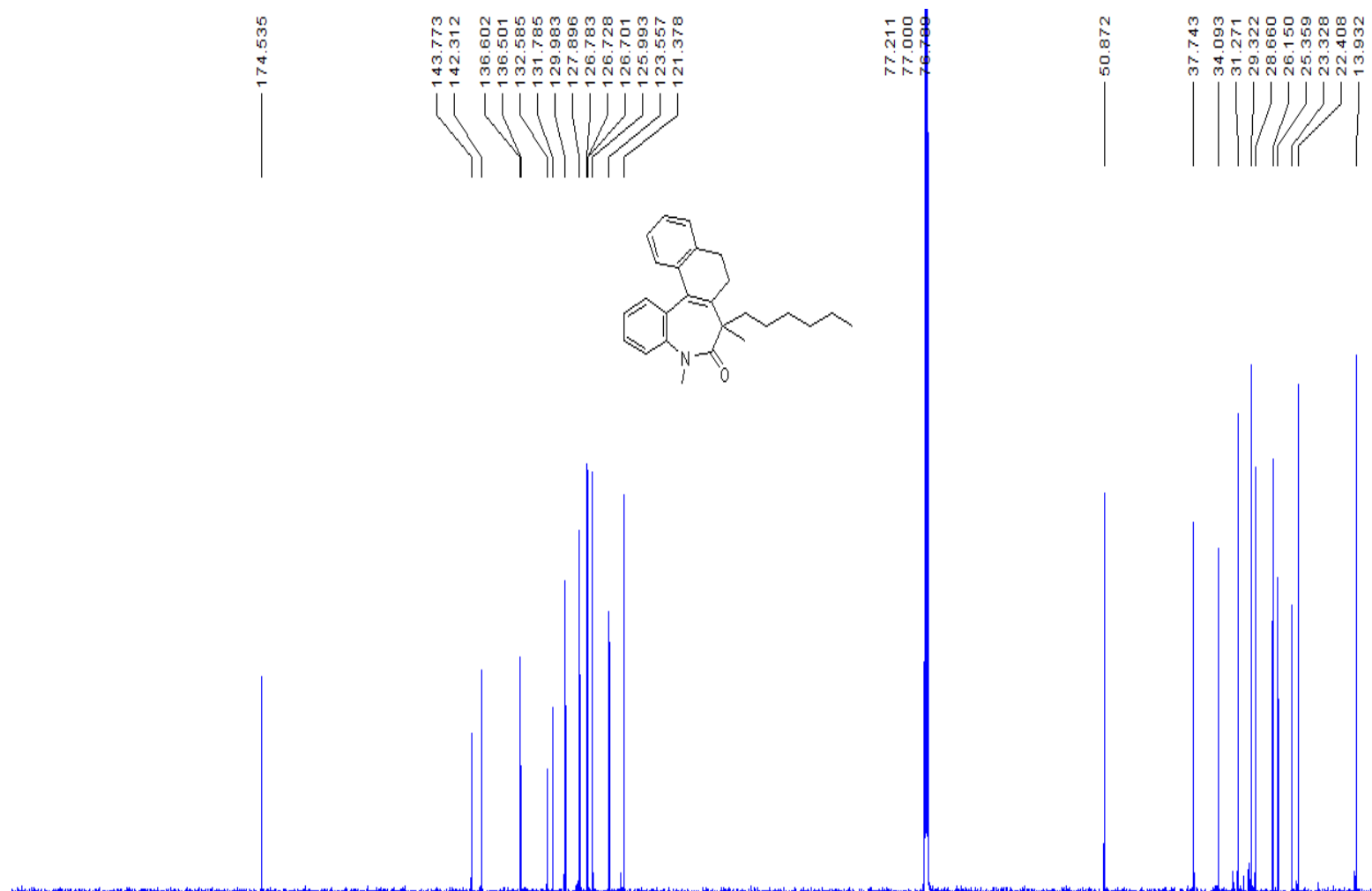

3q $\left({ }^{13} \mathrm{C} \mathrm{NMR,}, 150 \mathrm{MHz}, \mathrm{CDCl}_{3}\right)$ 


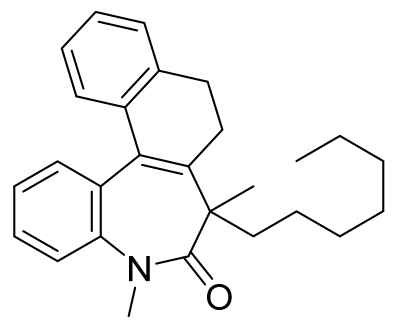

5,7-Dimethyl-7-heptyl-5,7,8,9-tetrahydro-6H-benzo[b]naphtho[1,2-d]azepin-6-one (3r)

Pale green oil, $58 \mathrm{mg}, 75 \%$ yield; Eluent: PE/EA = 10/1. ${ }^{1} \mathrm{H}$ NMR (400 $\left.\mathrm{MHz}, \mathrm{CDCl}_{3}, \mathrm{TMS}\right) \delta$ 7.37-7.33 (m, 1H), 7.30-7.24 (m, 2H), 7.20-7.08 (m, 4H), $6.96(\mathrm{~d}, J=7.6 \mathrm{~Hz}, 1 \mathrm{H}), 3.41(\mathrm{~s}, 3 \mathrm{H})$, 2.87-2.68 (m, 3H), $2.24\left(\mathrm{td}, J_{1}=6.4 \mathrm{~Hz}, J_{2}=16.8 \mathrm{~Hz}, 1 \mathrm{H}\right), 1.52(\mathrm{~s}, 3 \mathrm{H}), 1.12-0.90(\mathrm{~m}, 10 \mathrm{H})$, 0.83-0.80 (m, 5H); ${ }^{13} \mathrm{C}$ NMR (100 MHz, $\mathrm{CDCl}_{3}$, TMS) $\delta 174.5,143.8,142.3,136.6,136.5,132.6$, $131.8,130.0,127.9,126.8,126.73,126.70,126.0,123.5,121.4,50.9,37.7,34.1,31.6,29.6,28.7$, 28.6, 26.1, 25.4, 23.3, 22.5, 14.0; IR $\left(\mathrm{CH}_{2} \mathrm{Cl}_{2}\right): v 2932,2918,2909,1648,1494,1486,1447,1379$, 1255, 1081, 1059, 800, 763, 751, 699, $669 \mathrm{~cm}^{-1}$; HRMS (ESI) m/z: $(\mathrm{M}+\mathrm{H})^{+}$Calcd. for $\mathrm{C}_{27} \mathrm{H}_{34} \mathrm{NO}$ 388.2635; found 388.2628 . 


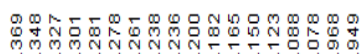

uMW W.

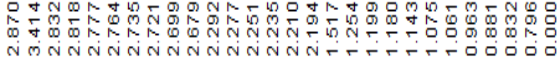

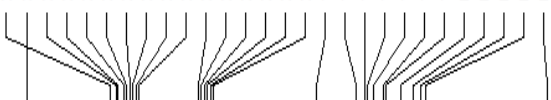

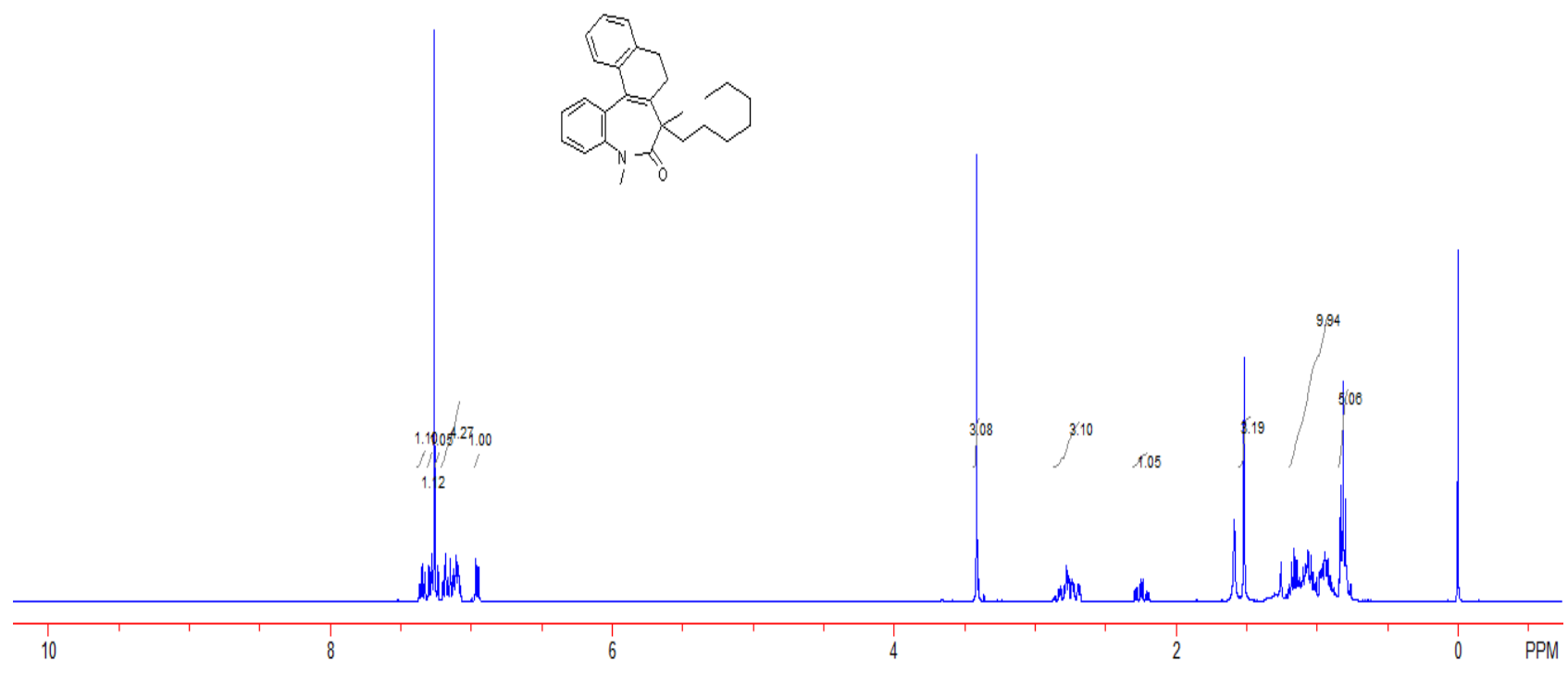

3r $\left({ }^{1} \mathrm{H}\right.$ NMR, $\left.400 \mathrm{MHz}, \mathrm{CDCl}_{3}\right)$
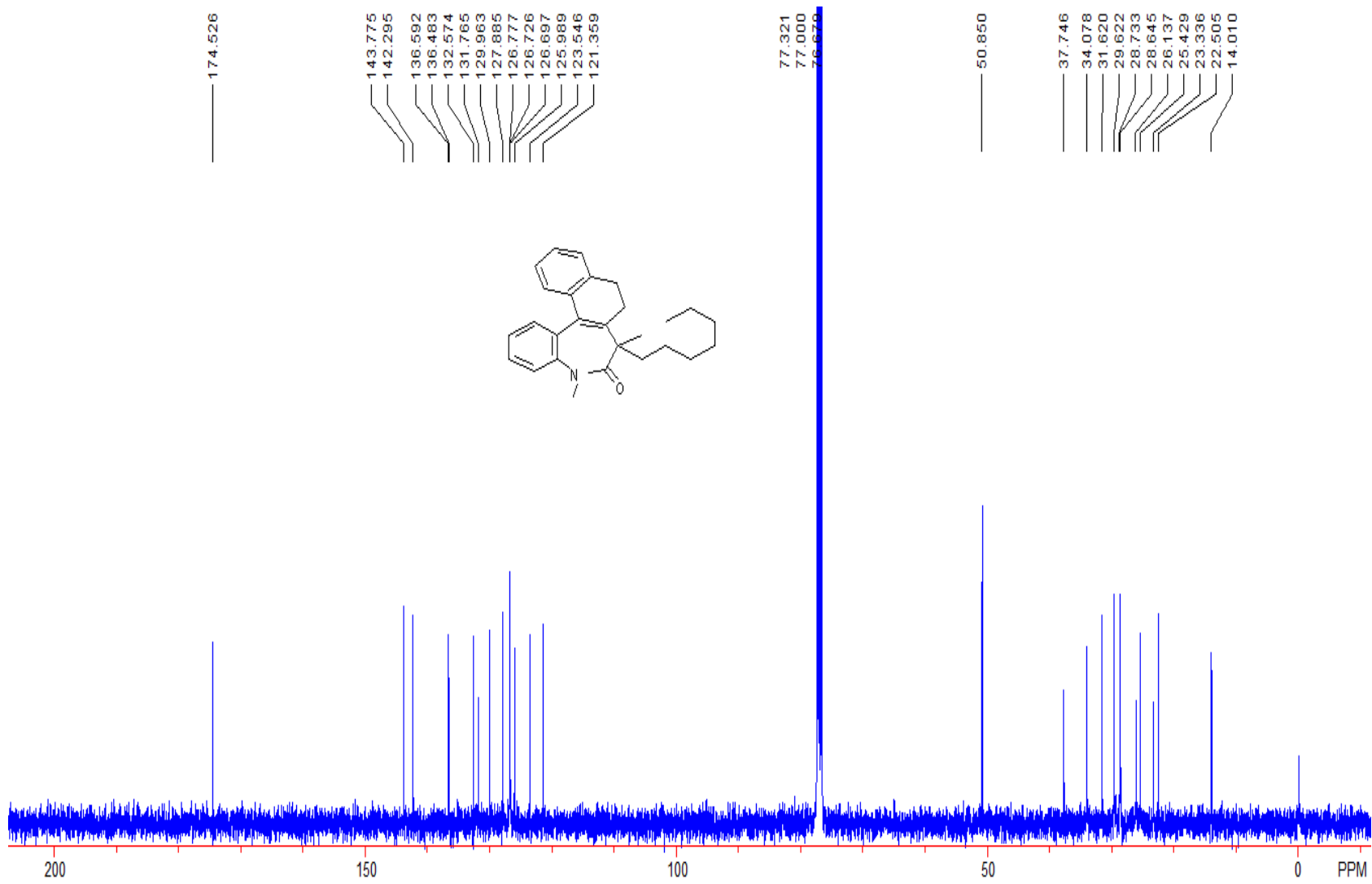

3r $\left({ }^{1} \mathrm{H}\right.$ NMR, $\left.400 \mathrm{MHz}, \mathrm{CDCl}_{3}\right)$ 


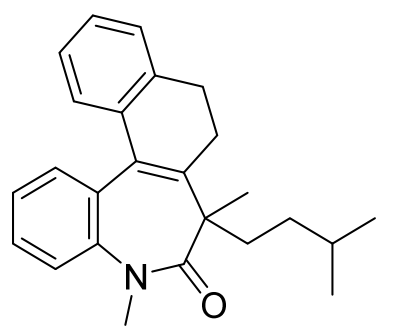

5,7-Dimethyl-7-isopentyl-5,7,8,9-tetrahydro-6H-benzo[b]naphtho[1,2-d]azepin-6-one (3s)

Pale green oil, $55 \mathrm{mg}, 77 \%$ yield; Eluent: PE/EA = 10/1. ${ }^{1} \mathrm{H}$ NMR (400 $\left.\mathrm{MHz}, \mathrm{CDCl}_{3}, \mathrm{TMS}\right) \delta$ 7.36-7.32 (m, 1H), 7.31-7.25 (m, 2H), 7.20-7.17 (m, 1H), 7.15-7.08 (m, 3H), 6.96 (d, J = 7.6 Hz, $1 \mathrm{H}), 3.42(\mathrm{~s}, 3 \mathrm{H}), 2.84-2.69(\mathrm{~m}, 3 \mathrm{H}), 2.25\left(\mathrm{td}, J_{1}=6.4 \mathrm{~Hz}, J_{2}=16.8 \mathrm{~Hz}, 1 \mathrm{H}\right), 1.52(\mathrm{~s}, 3 \mathrm{H})$, 1.05-1.00 (m, 4H), 0.83-0.81 (m, 1H), $0.53(\mathrm{~d}, J=6.0 \mathrm{~Hz}, 6 \mathrm{H}) ;{ }^{13} \mathrm{C} \mathrm{NMR}\left(150 \mathrm{MHz}, \mathrm{CDCl}_{3}, \mathrm{TMS}\right)$ $\delta 174.5,143.7,142.3,136.6,136.5,132.5,131.8,130.1,127.9,126.8,126.73,126.71,126.0,123.5$, 121.4, 50.7, 37.8, 34.5, 32.0, 28.7, 28.1, 26.2, 23.4, 22.3, 22.2; IR $\left(\mathrm{CH}_{2} \mathrm{Cl}_{2}\right): v 2943,2919,1648$, 1597, 1494, 1451, 1329, 1164, 1200, 1096, 1079, 765, 749, 704, 691, $670 \mathrm{~cm}^{-1}$; HRMS (ESI) m/z: $(\mathrm{M}+\mathrm{H})^{+}$Calcd. for $\mathrm{C}_{25} \mathrm{H}_{30} \mathrm{NO} 360.2322$; found 360.2316. 


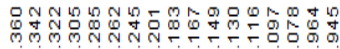

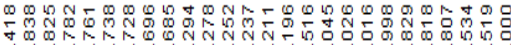

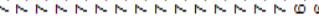

.

(n)

แUUHUHJ) LUHH
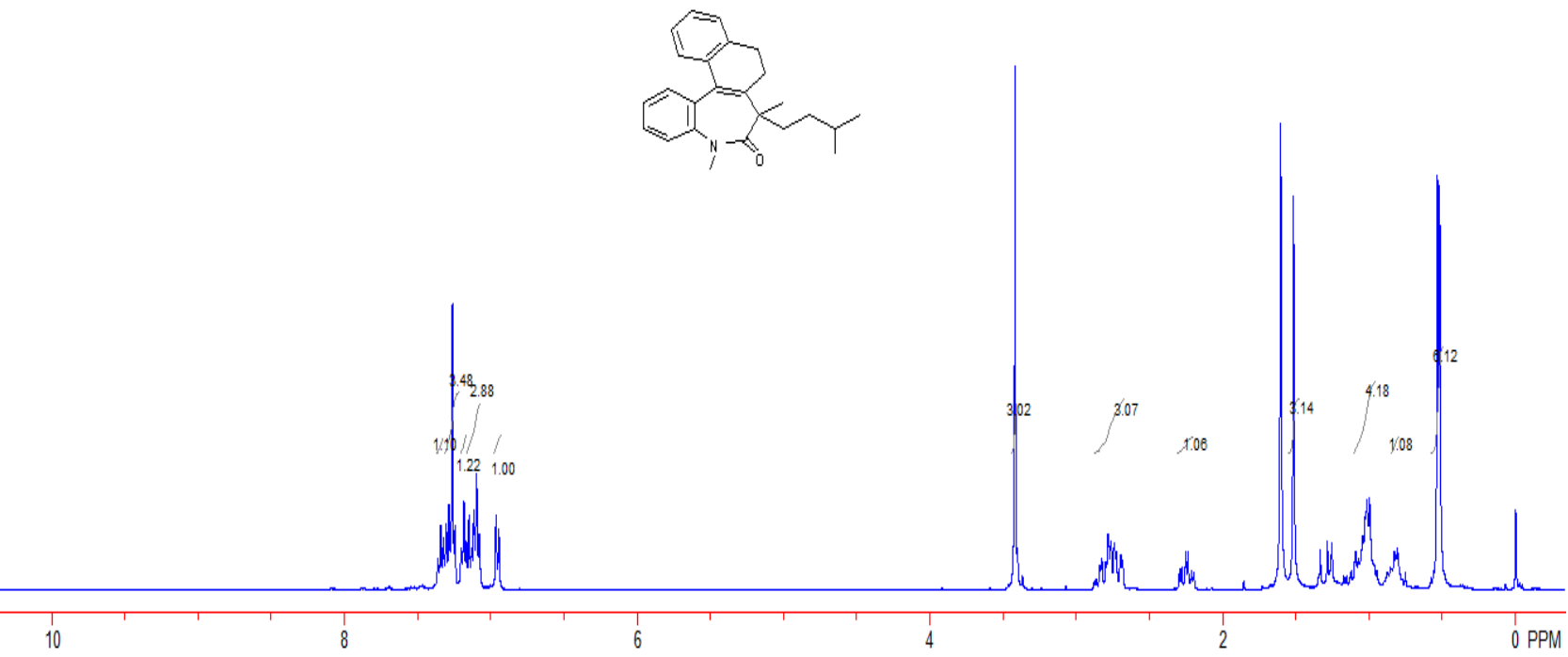

3s $\left({ }^{1} \mathrm{H} \mathrm{NMR}, 400 \mathrm{MHz}, \mathrm{CDCl}_{3}\right)$
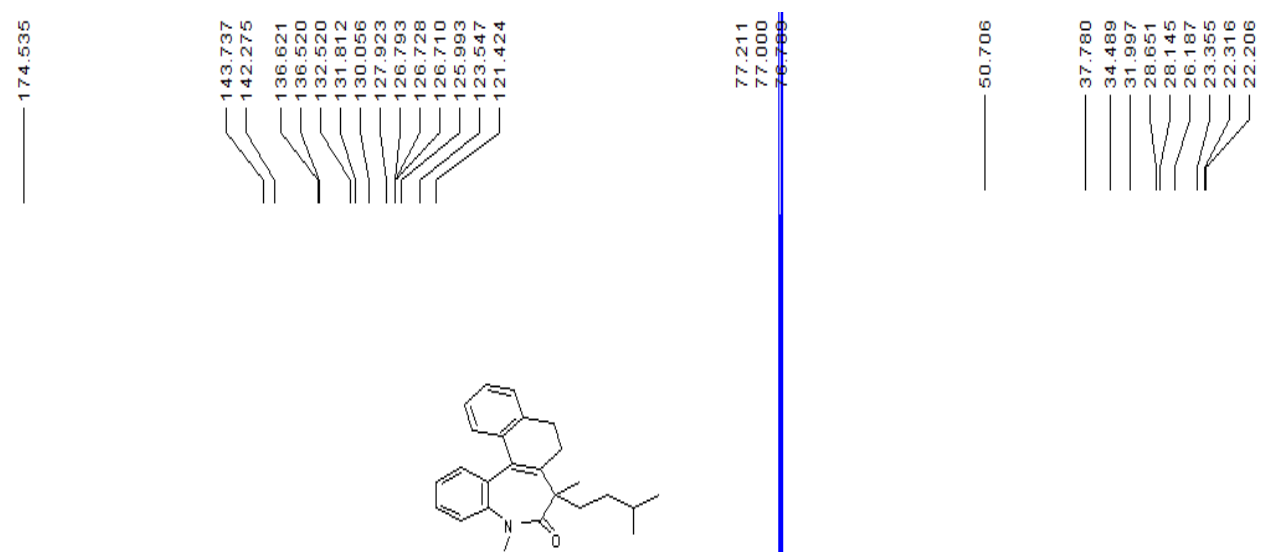

3s $\left({ }^{13} \mathrm{C} \mathrm{NMR,} 150 \mathrm{MHz}, \mathrm{CDCl}_{3}\right)$ 


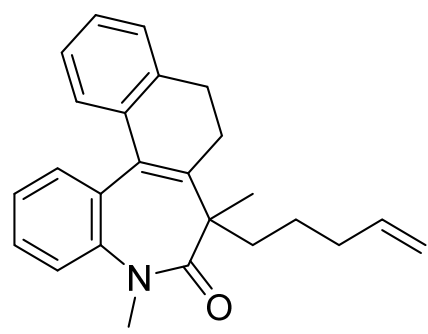

5,7-Dimethyl-7-(pent-4-en-1-yl)-5,7,8,9-tetrahydro-6H-benzo[b]naphtho[1,2-d]azepin-6-one (3t)

Pale green oil, $51 \mathrm{mg}, 72 \%$ yield; Eluent: PE/EA = 10/1. ${ }^{1} \mathrm{H}$ NMR (400 $\left.\mathrm{MHz}, \mathrm{CDCl}_{3}, \mathrm{TMS}\right) \delta$ 7.37-7.34 (m, 1H), 7.30-7.29 (m, 1H), 7.25-7.24 (m, 1H), 7.19-7.18 (m, 1H), 7.16-7.08 (m, 3H), $6.96(\mathrm{~d}, J=7.8 \mathrm{~Hz}, 1 \mathrm{H}), 5.47-5.40(\mathrm{~m}, 1 \mathrm{H}), 4.78-4.74(\mathrm{~m}, 2 \mathrm{H}), 3.41(\mathrm{~s}, 3 \mathrm{H}), 2.84-2.69(\mathrm{~m}, 3 \mathrm{H})$, $2.25\left(\mathrm{td}, J_{1}=6.4 \mathrm{~Hz}, J_{2}=16.8 \mathrm{~Hz}, 1 \mathrm{H}\right), 1.61-1.57(\mathrm{~m}, 2 \mathrm{H}), 1.52(\mathrm{~s}, 3 \mathrm{H}), 1.13-1.00(\mathrm{~m}, 4 \mathrm{H}) ;{ }^{13} \mathrm{C}$ NMR (150 MHz, $\left.\mathrm{CDCl}_{3}, \mathrm{TMS}\right) \delta 174.3,143.6,142.3,138.2,136.6,136.5,132.6,131.9,130.0$, $128.0,126.8,126.0,123.6,121.4,114.5,50.8,37.8,33.8,28.6,26.1,24.9,23.4,22.7 ; \mathrm{IR}\left(\mathrm{CH}_{2} \mathrm{Cl}_{2}\right)$ : $v 2936,2914,1644,1597,1449,1389,1360,1251,1197,1145,1114,1065,876,767,750,729,669$ $\mathrm{cm}^{-1}$; HRMS (ESI) m/z: $(\mathrm{M}+\mathrm{H})^{+}$Calcd. for $\mathrm{C}_{25} \mathrm{H}_{28} \mathrm{NO} 358.2165$; found 358.2160. 

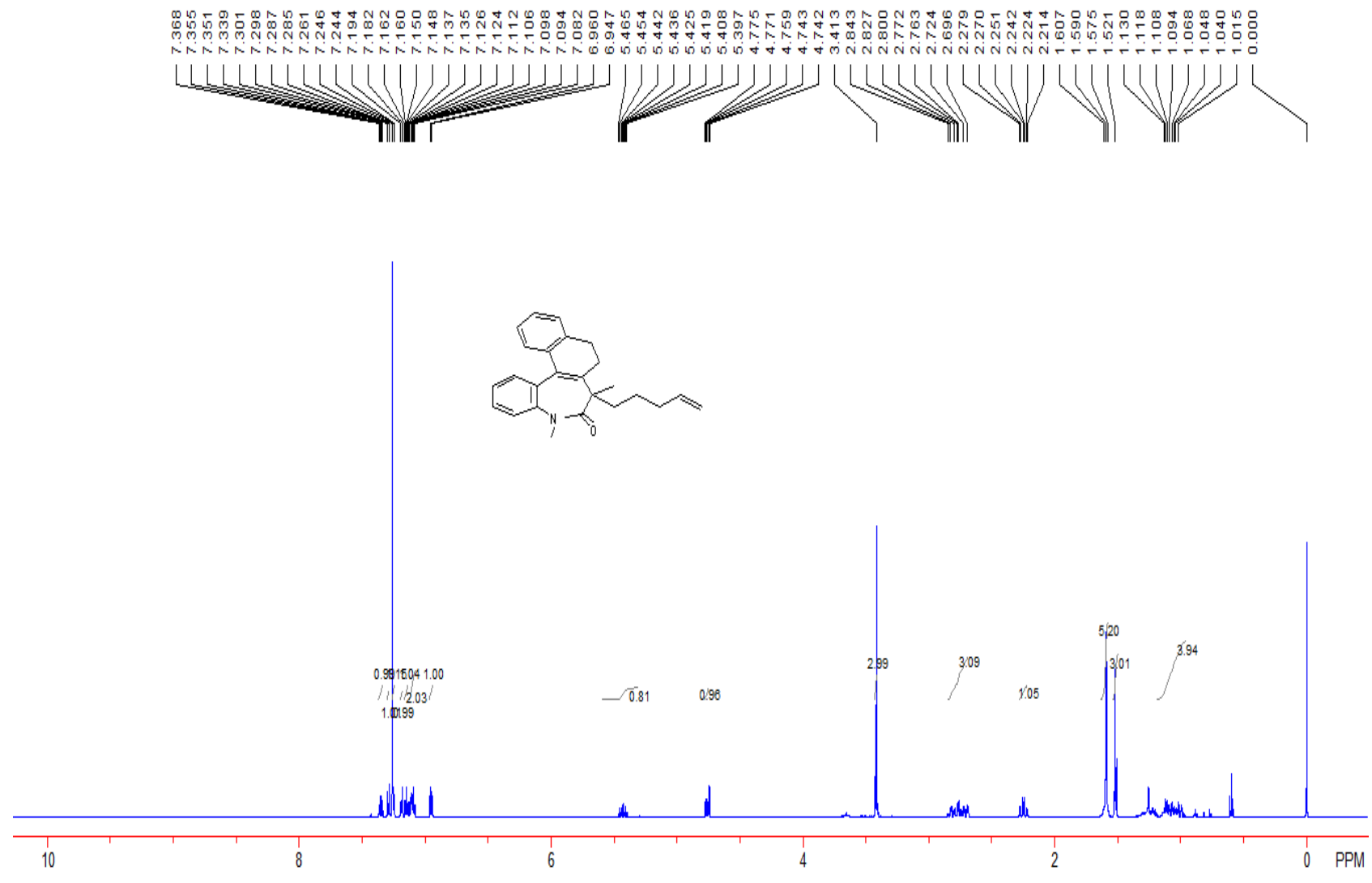

3t ( ${ }^{1} \mathrm{H}$ NMR, $\left.400 \mathrm{MHz}, \mathrm{CDCl}_{3}\right)$
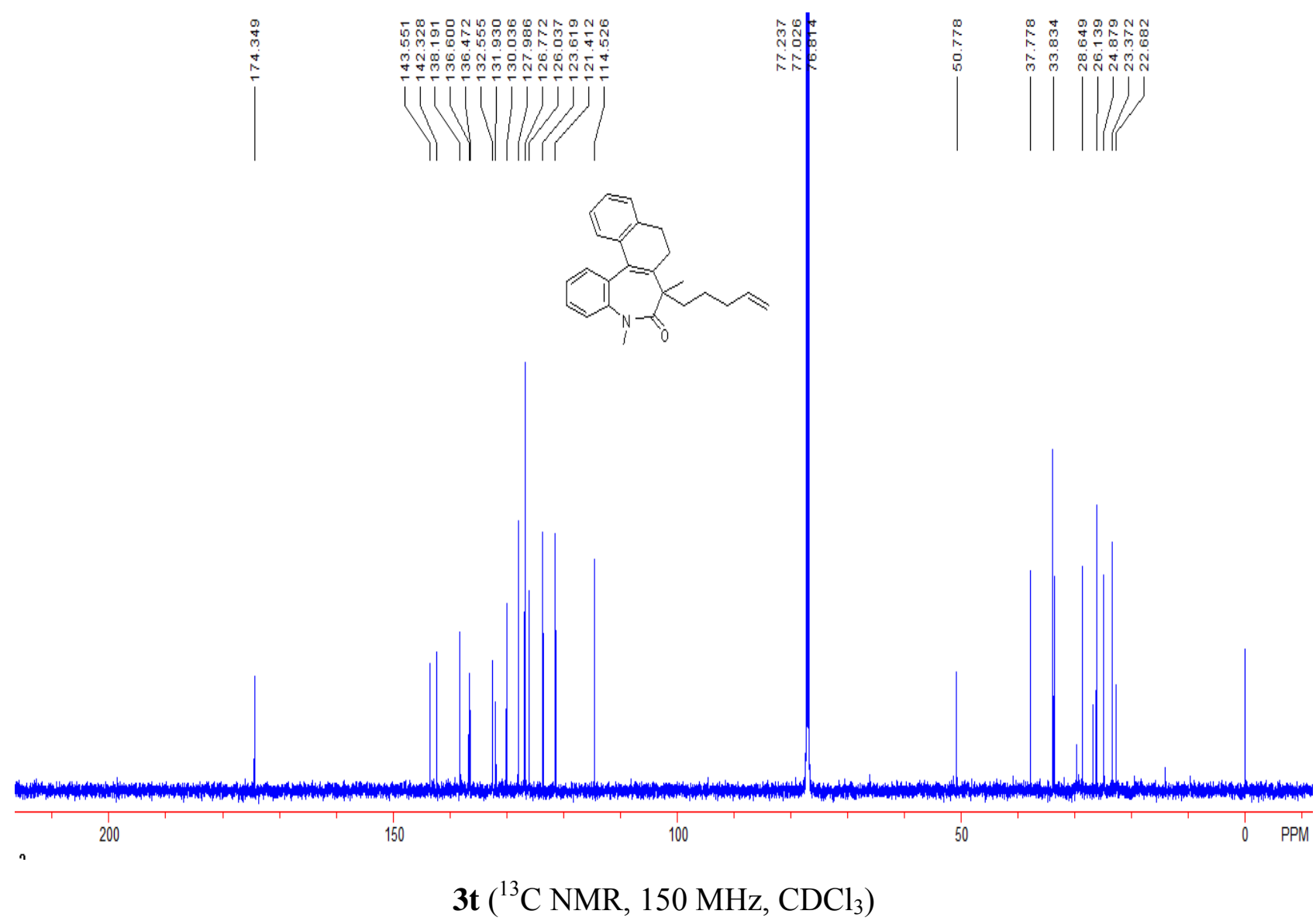


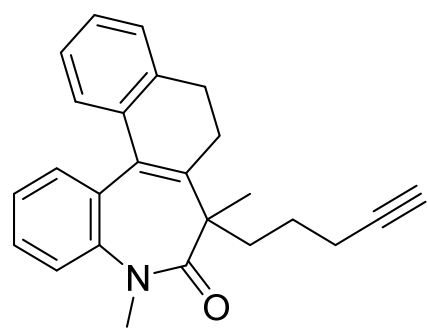

5,7-Dimethyl-7-(pent-4-yn-1-yl)-5,7,8,9-tetrahydro-6H-benzo[b]naphtho[1,2-d]azepin-6-one (3u)

Pale green oil, $55 \mathrm{mg}, 78 \%$ yield; Eluent: PE/EA = 10/1. ${ }^{1} \mathrm{H}$ NMR (400 $\left.\mathrm{MHz}, \mathrm{CDCl}_{3}, \mathrm{TMS}\right) \delta$ 7.37-7.34 (m, 1H), 7.31-7.26 (m, 2H), 7.20-7.17 (m, 1H), 7.15-7.08 (m, 3H), $6.95(\mathrm{~d}, J=7.6 \mathrm{~Hz}$, $1 \mathrm{H}), 3.42(\mathrm{~s}, 3 \mathrm{H}), 2.82-2.69(\mathrm{~m}, 3 \mathrm{H}), 2.25\left(\mathrm{td}, J_{1}=6.4 \mathrm{~Hz}, J_{2}=16.4 \mathrm{~Hz}, 1 \mathrm{H}\right), 1.76-1.72(\mathrm{~m}, 3 \mathrm{H})$, $1.54(\mathrm{~s}, 3 \mathrm{H}), 1.25-1.10(\mathrm{~m}, 4 \mathrm{H}) ;{ }^{13} \mathrm{C} \mathrm{NMR}\left(150 \mathrm{MHz}, \mathrm{CDCl}_{3}, \mathrm{TMS}\right) \delta 174.0,143.1,142.2,136.5$, 136.4, 132.4, 132.1, 130.1, 128.1, 126.83, 126.77, 126.0, 123.7, 121.4, 83.7, 68.4, 50.5, 37.8, 33.4, 28.6, 26.1, 24.7, 23.3, 18.6; IR $\left(\mathrm{CH}_{2} \mathrm{Cl}_{2}\right): v$ 3309, 2958, 2921, 2916, 2208, 1644, 1499, 1494, 1352, 1247, 1044, 876, 765, 729, 699, 696, 678, $669 \mathrm{~cm}^{-1}$; HRMS (ESI) m/z: $(\mathrm{M}+\mathrm{H})^{+}$Calcd. for $\mathrm{C}_{25} \mathrm{H}_{26} \mathrm{NO} 356.2009$; found 356.2006. 


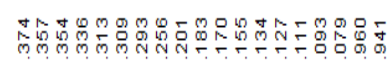

uiviuu.

al)

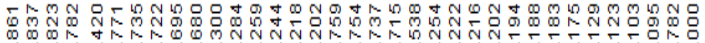

anan mannasanana...................

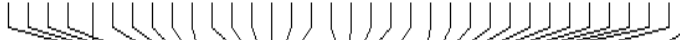

$=\infty$<smiles>C#CCCCC1(CCC#C)C(=O)N(C)c2ccccc2C2=C1c1ccccc1CC2</smiles>

2,72271

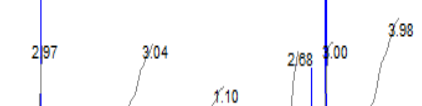

Wh

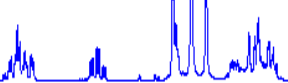

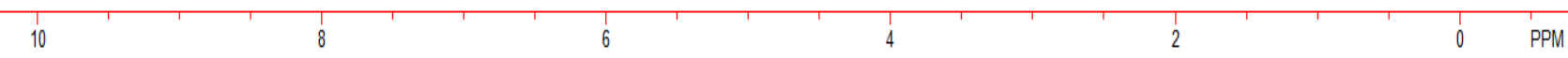

3u $\left({ }^{1} \mathrm{H}\right.$ NMR, $\left.400 \mathrm{MHz}, \mathrm{CDCl}_{3}\right)$
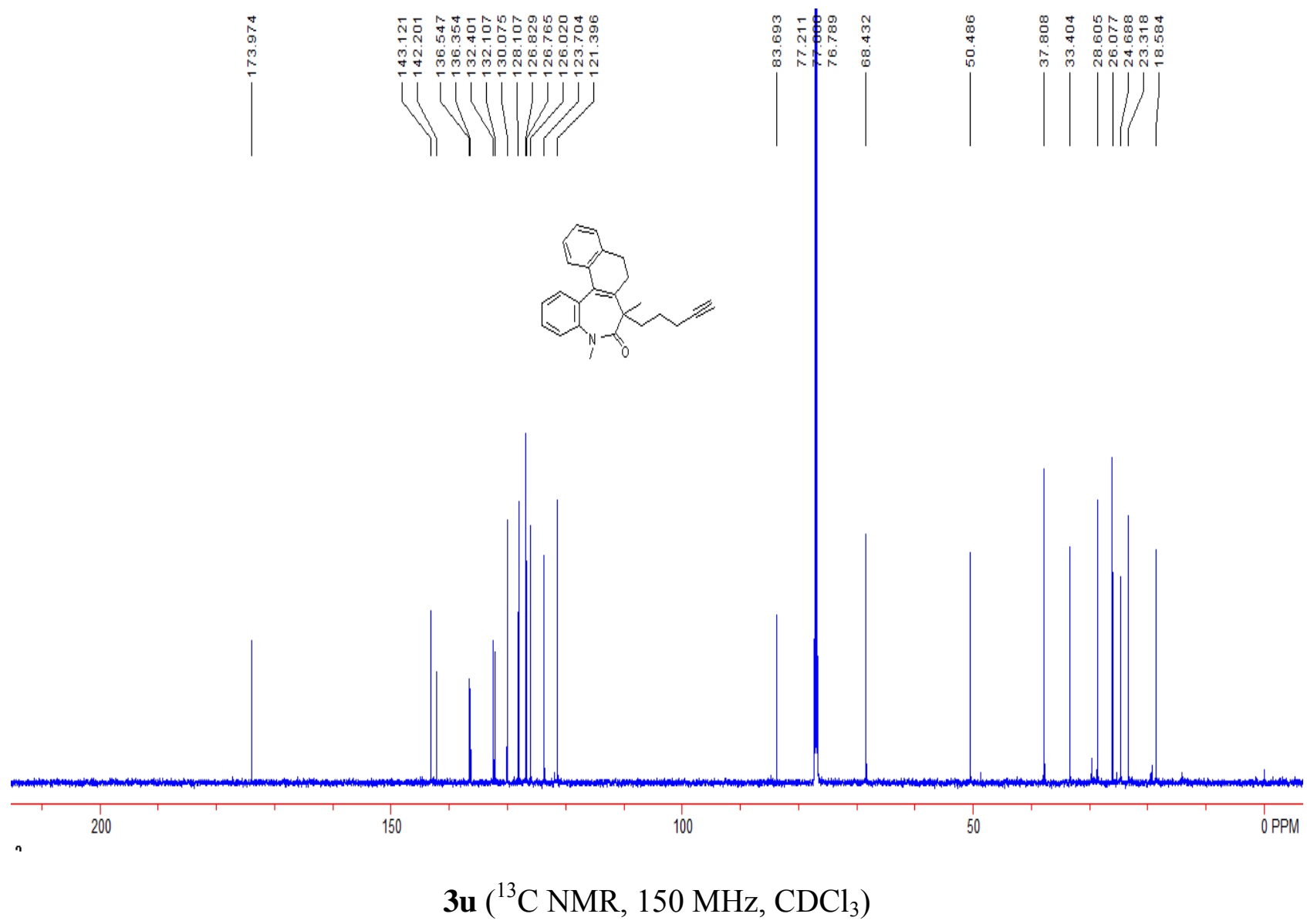


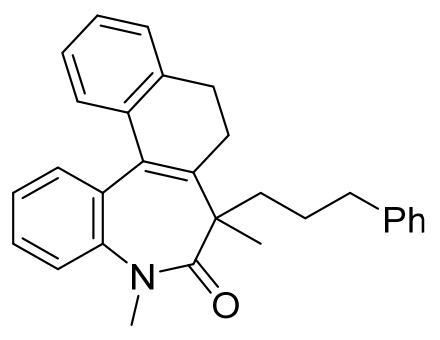

5,7-Dimethyl-7-(3-phenylpropyl)-5,7,8,9-tetrahydro-6H-benzo[b]naphtho[1,2-d]azepin-6-one (3v)

Pale green oil, $64 \mathrm{mg}, 78 \%$ yield; Eluent: PE/EA = 10/1. ${ }^{1} \mathrm{H}$ NMR (400 $\left.\mathrm{MHz}, \mathrm{CDCl}_{3}, \mathrm{TMS}\right) \delta$ 7.36-7.31 (m, 1H), 7.29-7.24 (m, 2H), 7.22-7.17 (m, 4H), 7.15-7.07 (m, 3H), 7.00-6.91 (m, 3H), $3.40(\mathrm{~s}, 3 \mathrm{H}), 2.84-2.67(\mathrm{~m}, 3 \mathrm{H}), 2.24\left(\mathrm{td}, J_{1}=6.0 \mathrm{~Hz}, J_{2}=16.4 \mathrm{~Hz}, 1 \mathrm{H}\right), 2.13-2.09(\mathrm{~m}, 2 \mathrm{H})$, 1.51-1.41 (m, 4H), 1.29-1.24 (m, 1H), 1.13-1.03 (m, 2H); ${ }^{13} \mathrm{C}$ NMR (150 MHz, CDCl 3 , TMS) $\delta$ $174.3,143.4,142.2,141.9,136.5,136.4,132.5,131.9,129.9,128.2,128.0,126.8,126.0,125.6$, 123.6, 121.3, 50.7, 37.7, 36.1, 34.0, 28.6, 27.4, 26.1, 23.4; IR $\left(\mathrm{CH}_{2} \mathrm{Cl}_{2}\right): v 2972,2925,1650,1492$, 1453, 1381, 1374, 1045, 879, 762, $668 \mathrm{~cm}^{-1}$; HRMS (ESI) m/z: $(\mathrm{M}+\mathrm{H})^{+}$Calcd. for $\mathrm{C}_{29} \mathrm{H}_{30} \mathrm{NO}$ 408.2322; found 408.2312. 


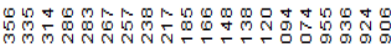

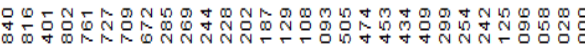

LLUUUUUHUJUJ

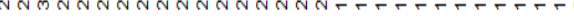

W.WWW WW
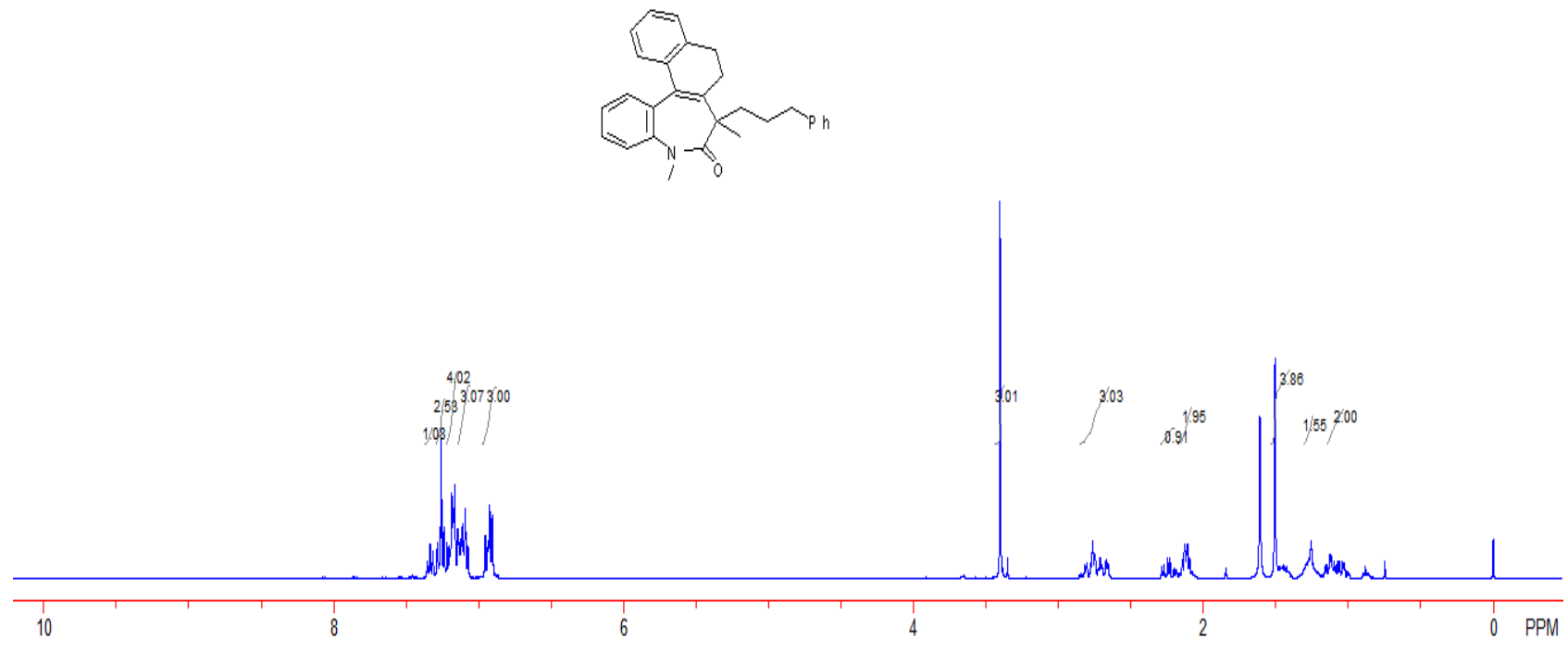

3v $\left({ }^{1} \mathrm{H}\right.$ NMR, $\left.400 \mathrm{MHz}, \mathrm{CDCl}_{3}\right)$
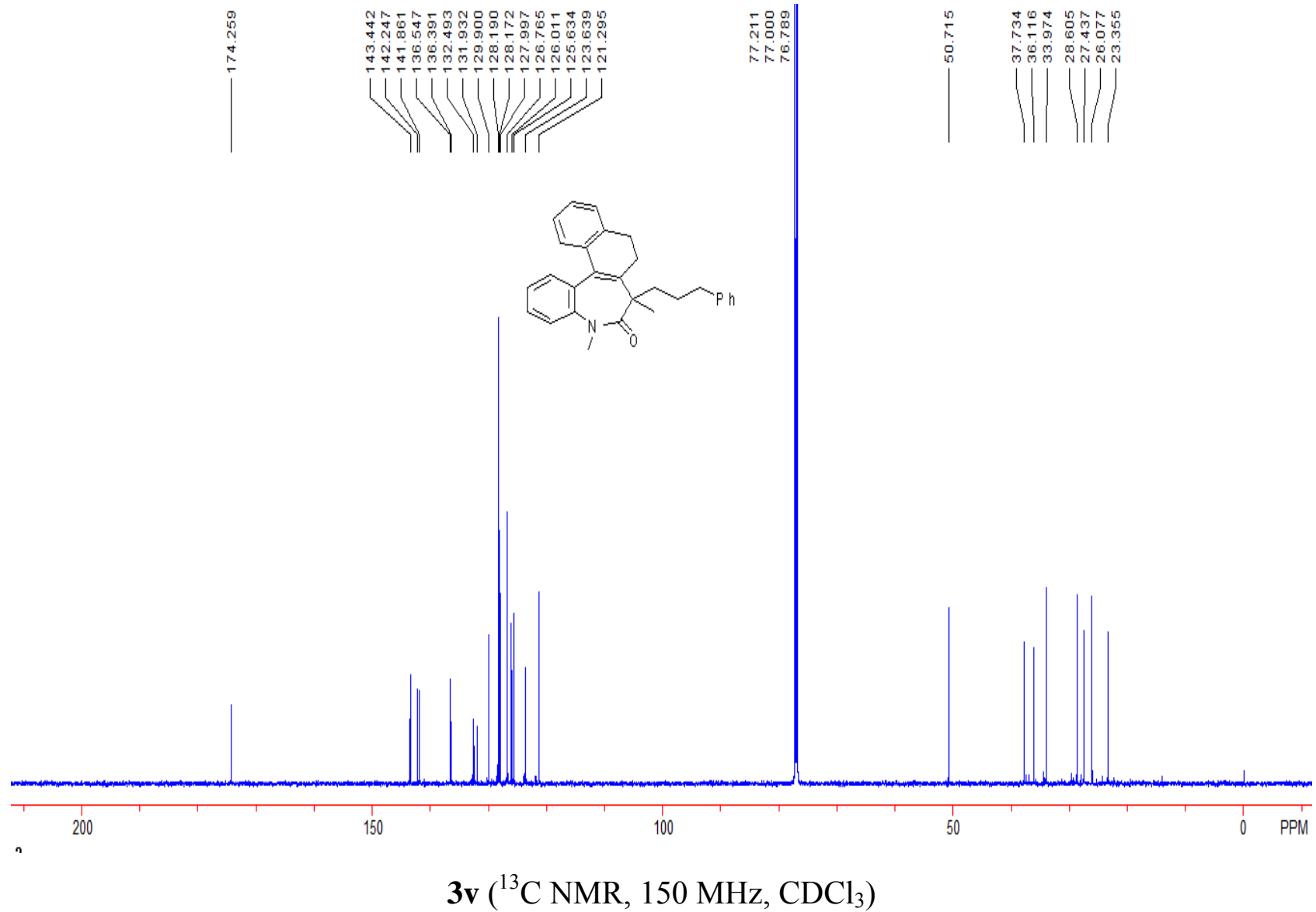


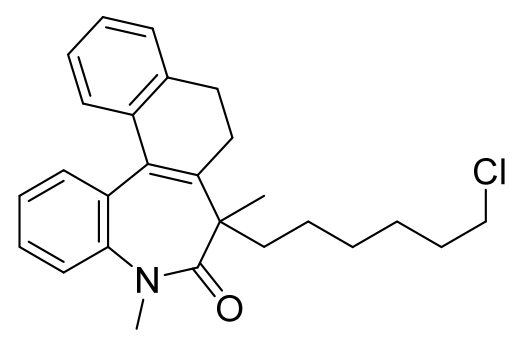

5,7-Dimethyl-7-(6-chlorohexyl)-5,7,8,9-tetrahydro-6H-benzo[b]naphtho[1,2-d]azepin-6-one (3w)

Pale green oil, $64 \mathrm{mg}, 83 \%$ yield; Eluent: PE/EA = 10/1. ${ }^{1} \mathrm{H}$ NMR $\left(600 \mathrm{MHz}, \mathrm{CDCl}_{3}, \mathrm{TMS}\right) \delta$ 7.37-7.35 (m, 1H), $7.30\left(\mathrm{dd}, J_{1}=1.2 \mathrm{~Hz}, J_{2}=7.8 \mathrm{~Hz}, 1 \mathrm{H}\right), 7.26-7.25(\mathrm{~m}, 1 \mathrm{H}), 7.20-7.18(\mathrm{~m}, 1 \mathrm{H})$, 7.16-7.08 (m, 3H), 6.95 (d, $J=7.2 \mathrm{~Hz}, 1 \mathrm{H}), 3.42-3.39(\mathrm{~m}, 5 \mathrm{H}), 2.82-2.69(\mathrm{~m}, 3 \mathrm{H}), 2.25\left(\mathrm{td}, J_{1}=6.0\right.$ $\left.\mathrm{Hz}, J_{2}=16.8 \mathrm{~Hz}, 1 \mathrm{H}\right), 1.59-1.55(\mathrm{~m}, 2 \mathrm{H}), 1.52(\mathrm{~s}, 3 \mathrm{H}), 1.14-1.05(\mathrm{~m}, 4 \mathrm{H}), 1.02-0.93(\mathrm{~m}, 2 \mathrm{H})$, 0.88-0.85 (m, 2H); ${ }^{13} \mathrm{C}$ NMR (150 MHz, $\mathrm{CDCl}_{3}$, TMS) $\delta 174.4,143.6,142.3,136.6,136.4,132.6$, $131.9,130.0,128.0,126.8,126.7,126.0,123.6,121.4,50.8,44.9,37.7,33.9,32.3,28.8,28.6,26.3$, 26.1, 25.2, 23.3; IR $\left(\mathrm{CH}_{2} \mathrm{Cl}_{2}\right): v$ 2969, 2930, 2924, 1646, 1494, 1352, 1209, 1054, 1042, 878, 765, 729, 699, 678, $668 \mathrm{~cm}^{-1}$; HRMS (ESI) m/z: $(\mathrm{M}+\mathrm{H})^{+}$Calcd. for $\mathrm{C}_{26} \mathrm{H}_{31} \mathrm{ClNO}$ 408.2089; found 408.2092 

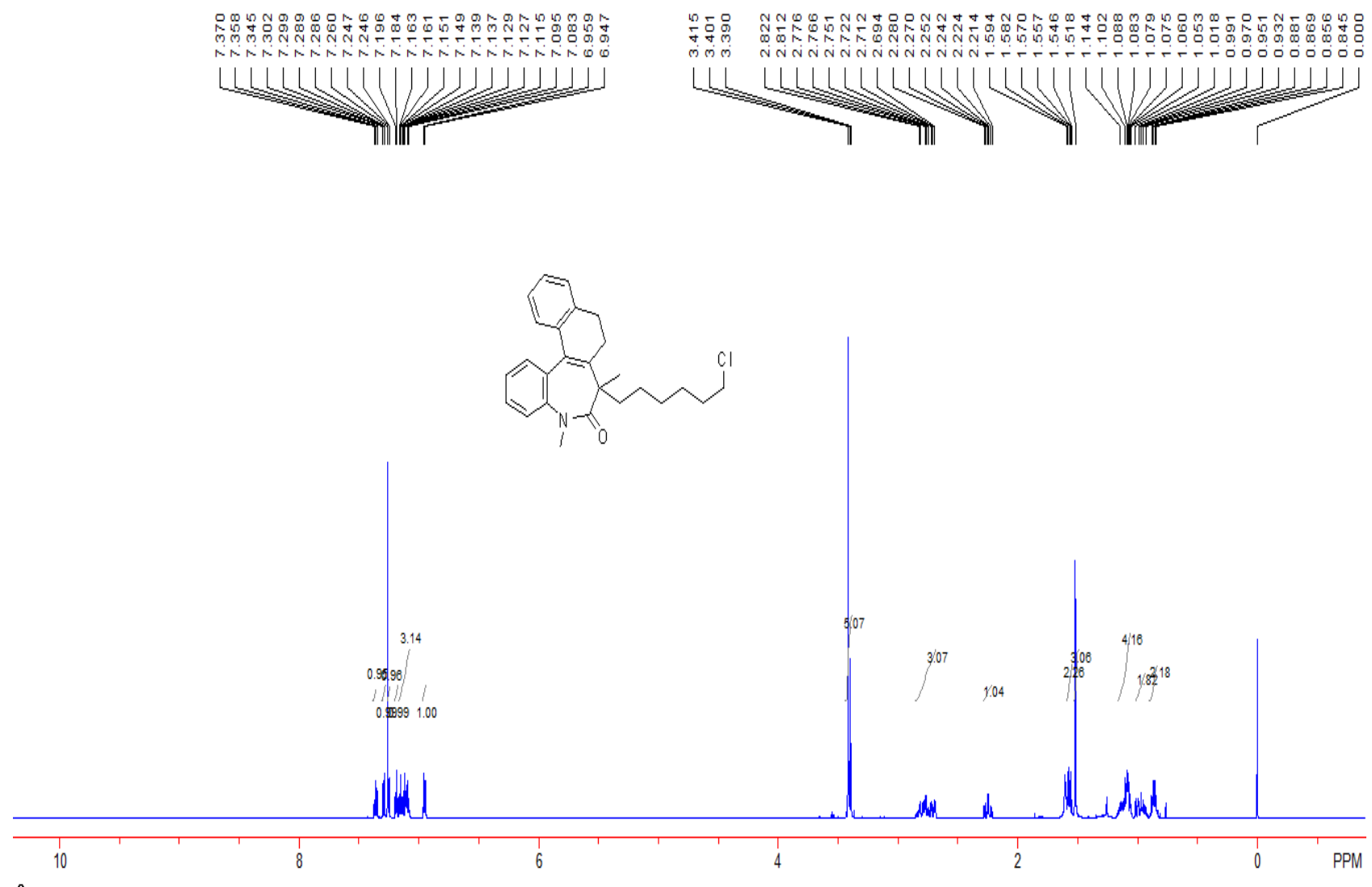

3w $\left({ }^{1} \mathrm{H}\right.$ NMR, $\left.400 \mathrm{MHz}, \mathrm{CDCl}_{3}\right)$
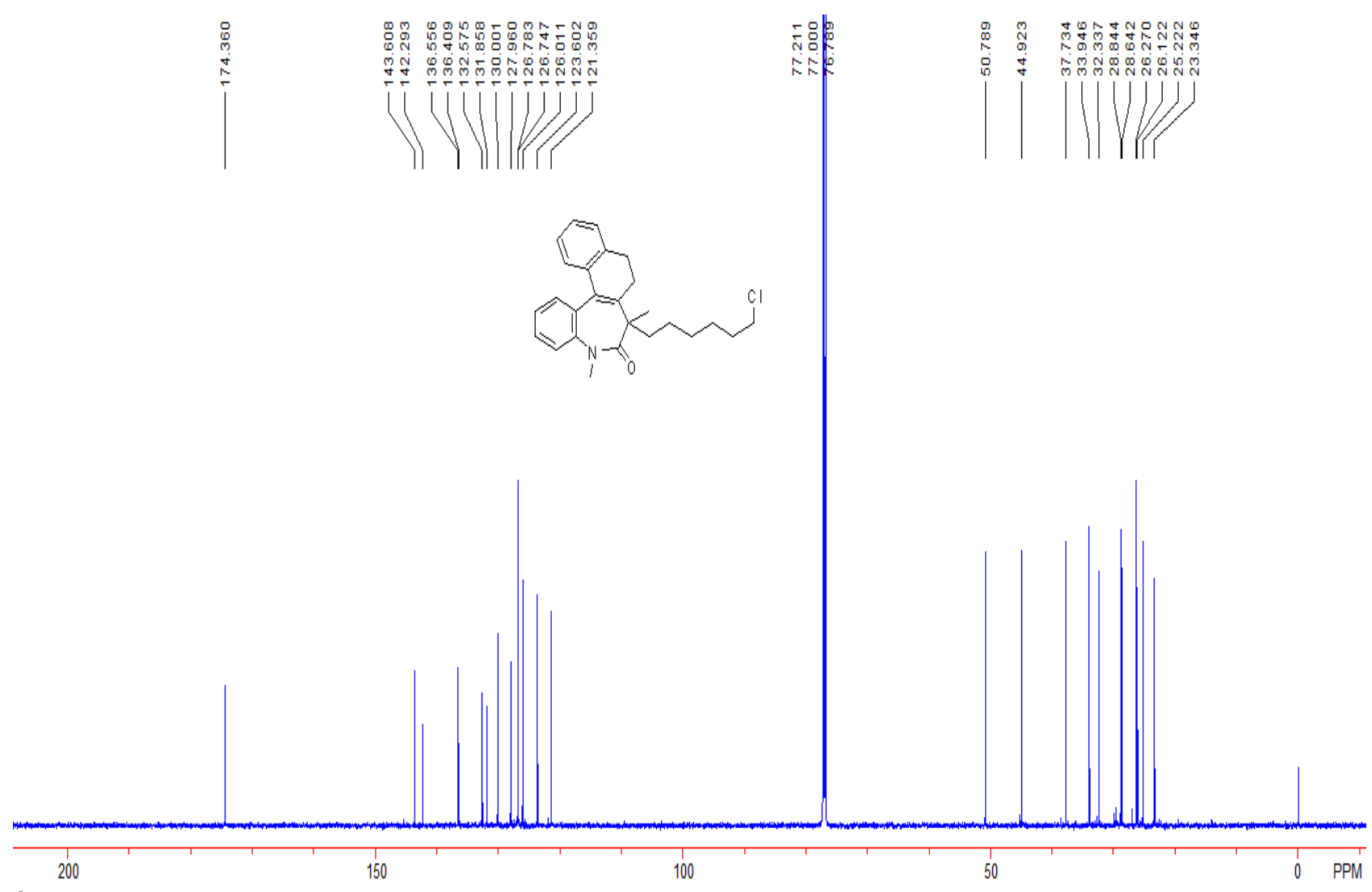

3w $\left({ }^{13} \mathrm{C} \mathrm{NMR,} 150 \mathrm{MHz}, \mathrm{CDCl}_{3}\right)$ 


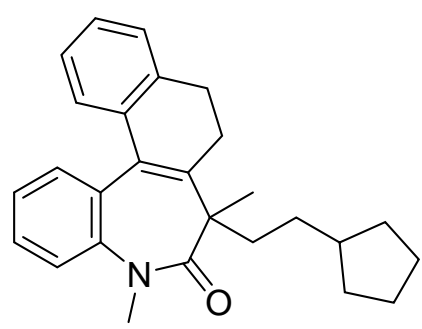

5,7-Dimethyl-7-(2-cyclopentylethyl)-5,7,8,9-tetrahydro-6H-benzo[b]naphtho[1,2-d]azepin-6-on e (3x)

Pale green oil, $59 \mathrm{mg}, 76 \%$ yield; Eluent: $\mathrm{PE} / \mathrm{EA}=10 / 1 .{ }^{1} \mathrm{H}$ NMR $\left(400 \mathrm{MHz}, \mathrm{CDCl}_{3}, \mathrm{TMS}\right) \delta$ 7.37-7.33 (m, 1H), 7.31-7.24 (m, 2H), 7.20-7.18 (m, 1H), 7.17-7.09 (m, 3H), 6.96 (d, J = 7.2 Hz, $1 \mathrm{H}), 3.42(\mathrm{~s}, 3 \mathrm{H}), 2.87-2.68(\mathrm{~m}, 3 \mathrm{H}), 2.24\left(\mathrm{td}, J_{1}=6.4 \mathrm{~Hz}, J_{2}=16.8 \mathrm{~Hz}, 1 \mathrm{H}\right), 1.52(\mathrm{~s}, 3 \mathrm{H})$, 1.42-1.25 (m, 7H), 1.11-0.96 (m, 4H), 0.68-0.65 (m, 2H); $\left.{ }^{13} \mathrm{C} \mathrm{NMR} \mathrm{(100} \mathrm{MHz,} \mathrm{CDCl}_{3}, \mathrm{TMS}\right) \delta$ $174.5,143.7,142.3,136.6,136.5,132.5,131.8,130.1,127.9,126.8,126.72,126.70,126.0,123.5$, $121.4,50.7,40.1,37.8,33.3,32.23,32.16,31.8,28.6,26.2,24.93,24.90,23.4 ; \mathrm{IR}\left(\mathrm{CH}_{2} \mathrm{Cl}_{2}\right): v 2980$, 2920, 2910, 1645, 1595, 1494, 1444, 1352, 1307, 1231, 1042, 765, 729, 699, 676, $665 \mathrm{~cm}^{-1}$; HRMS (ESI) m/z: $(\mathrm{M}+\mathrm{H})^{+}$Calcd. for $\mathrm{C}_{27} \mathrm{H}_{32} \mathrm{NO} 386.2478$; found 386.2475 . 

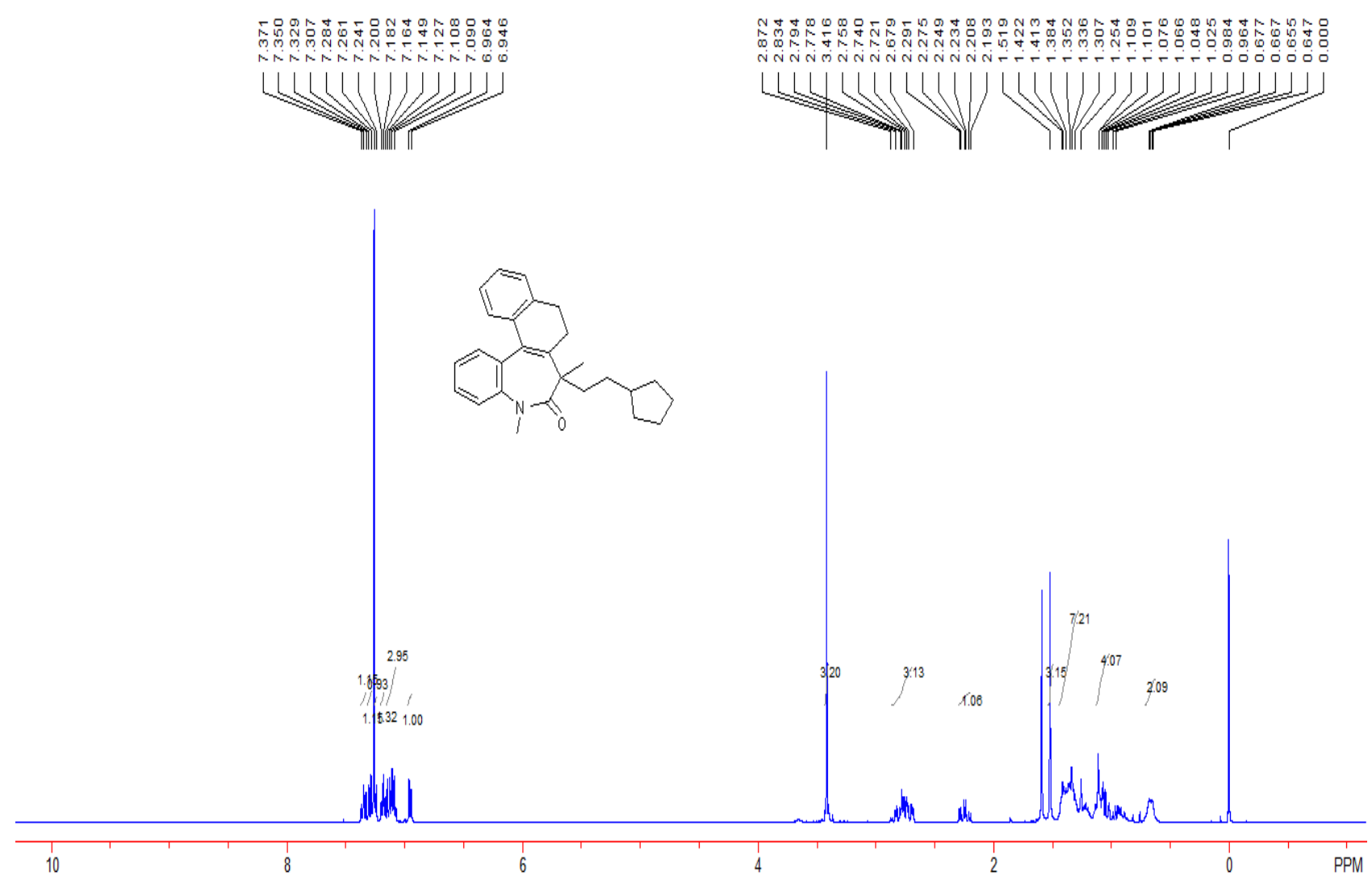

3x ( ${ }^{1} \mathrm{H}$ NMR, $\left.400 \mathrm{MHz}, \mathrm{CDCl}_{3}\right)$
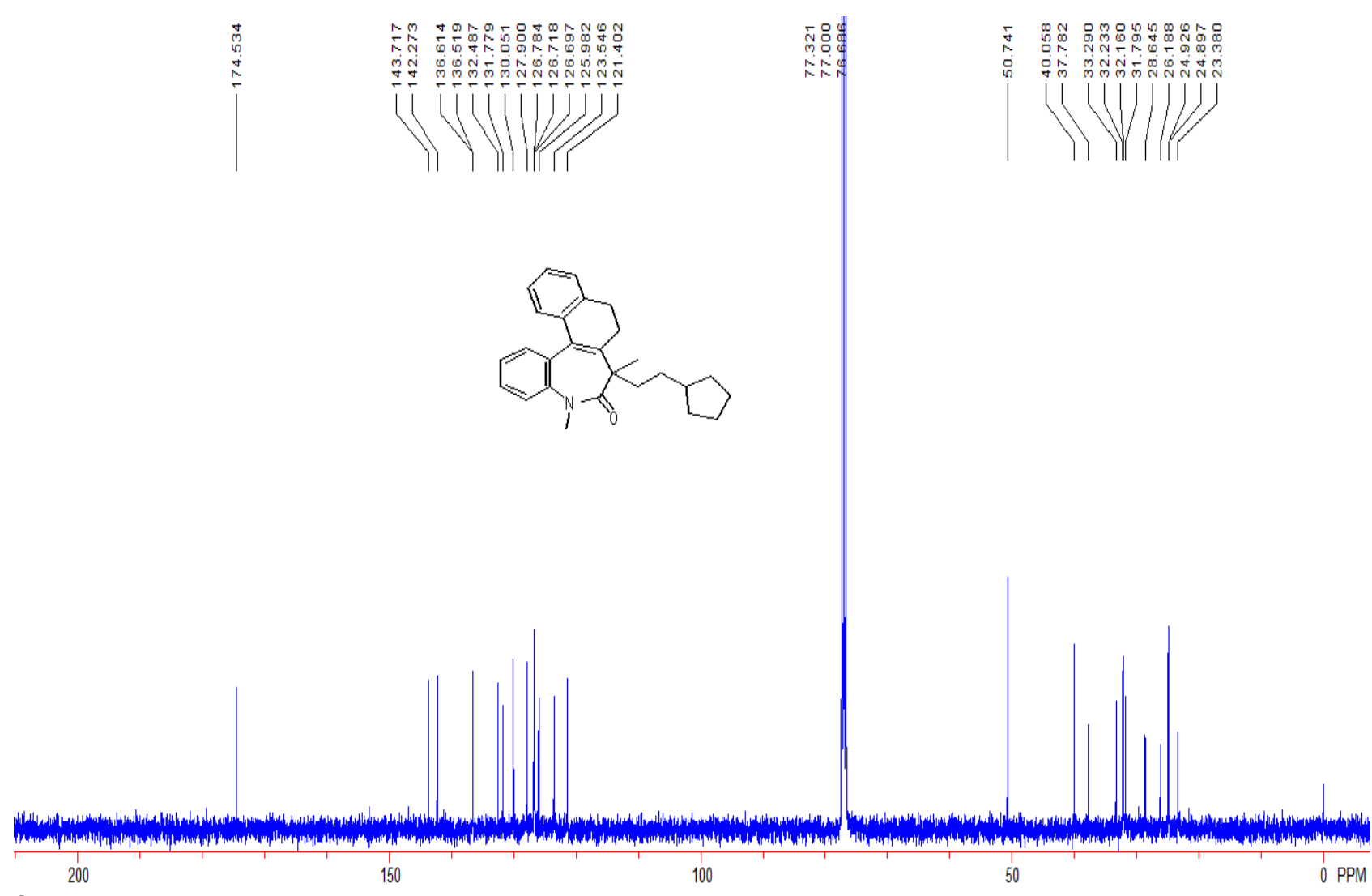

$3 \mathbf{x}\left({ }^{13} \mathrm{C} \mathrm{NMR}, 100 \mathrm{MHz}, \mathrm{CDCl}_{3}\right)$ 


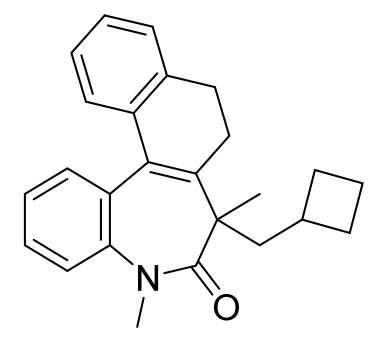

5,7-Dimethyl-7-cyclobutylmethyl-5,7,8,9-tetrahydro-6H-benzo[b]naphtho[1,2-d]azepin-6-one (3z)

Pale green oil, $52 \mathrm{mg}, 72 \%$ yield; Eluent: $\mathrm{PE} / \mathrm{EA}=10 / 1 .{ }^{1} \mathrm{H}$ NMR $\left(600 \mathrm{MHz}, \mathrm{CDCl}_{3}, \mathrm{TMS}\right) \delta$ 7.38-7.35 (m, 1H), $7.29\left(\mathrm{dd}, J_{1}=1.2 \mathrm{~Hz}, J_{2}=7.8 \mathrm{~Hz}, 1 \mathrm{H}\right), 7.26-7.24(\mathrm{~m}, 1 \mathrm{H}), 7.19-7.17(\mathrm{~m}, 1 \mathrm{H})$, 7.16-7.08 (m, 3H), $6.95(\mathrm{~d}, J=7.8 \mathrm{~Hz}, 1 \mathrm{H}), 3.41(\mathrm{~s}, 3 \mathrm{H}), 2.85-2.69(\mathrm{~m}, 3 \mathrm{H}), 2.23\left(\mathrm{td}, J_{1}=6.0 \mathrm{~Hz}\right.$, $\left.J_{2}=16.8 \mathrm{~Hz}, 1 \mathrm{H}\right), 2.16-2.11(\mathrm{~m}, 1 \mathrm{H}), 1.80-1.75(\mathrm{~m}, 2 \mathrm{H}), 1.53-1.49(\mathrm{~m}, 4 \mathrm{H}), 1.33-1.21(\mathrm{~m}, 4 \mathrm{H})$, 1.16-1.13 (m, 1H); ${ }^{13} \mathrm{C}$ NMR (150 MHz, $\mathrm{CDCl}_{3}$, TMS) $\delta 174.4,143.6,142.5,136.6,136.5,132.6$, $131.9,130.1,127.9,126.8,126.7,126.0,123.5,121.5,50.8,40.2,37.9,33.6,30.0,29.4,28.8,26.2$, 23.3, 19.0; IR $\left(\mathrm{CH}_{2} \mathrm{Cl}_{2}\right): v$ 2989, 2950, 2921, 1645, 1576, 1494, 1443, 1352, 1041, 765, 700, 729, 678, $669 \mathrm{~cm}^{-1}$; HRMS (ESI) m/z: (M+H) ${ }^{+}$Calcd. for $\mathrm{C}_{25} \mathrm{H}_{28} \mathrm{NO} 358.2165$; found 358.2160 . 


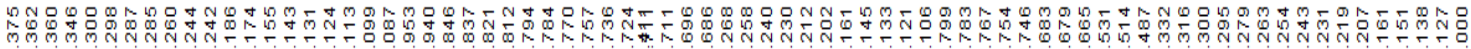
LQLLU

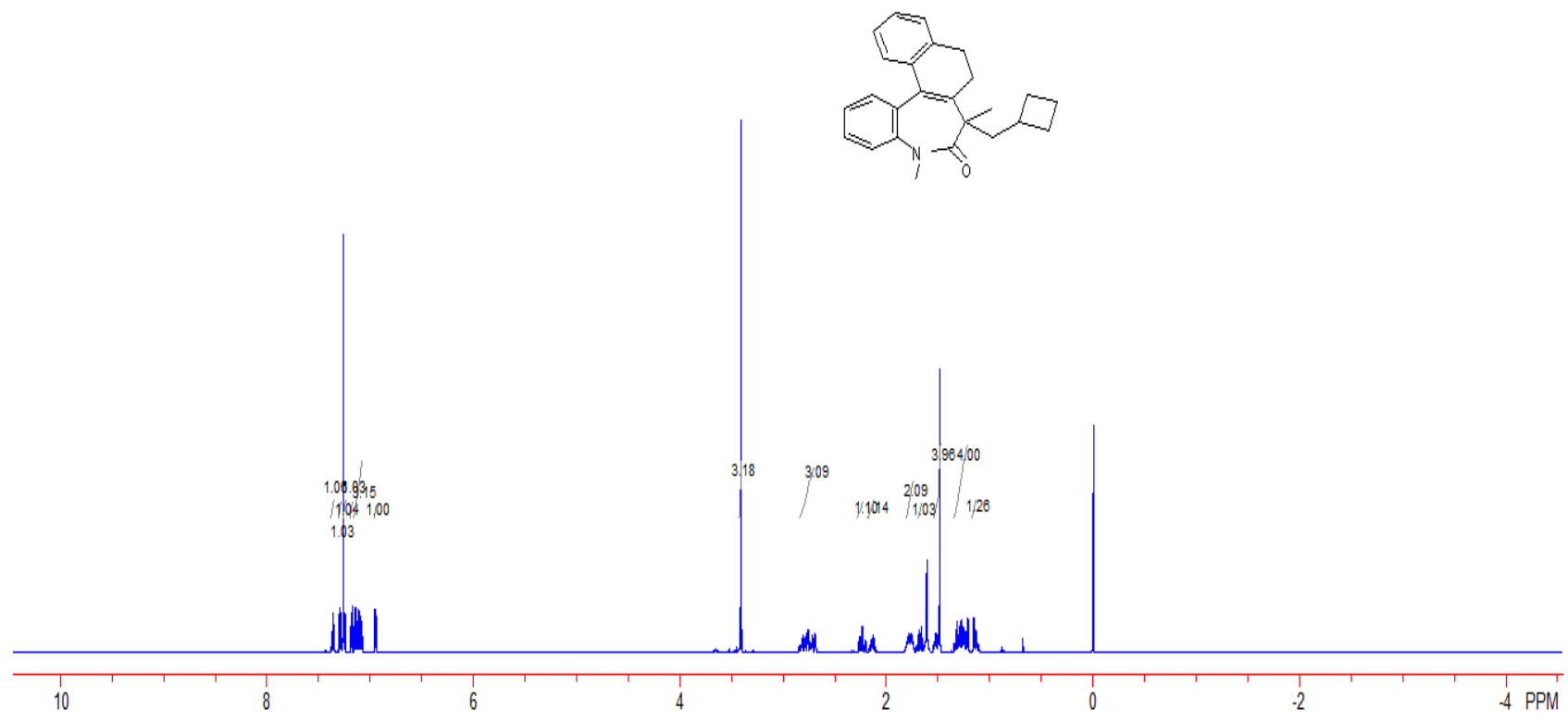

$3 z\left({ }^{1} \mathrm{H} \mathrm{NMR}, 600 \mathrm{MHz}, \mathrm{CDCl}_{3}\right)$
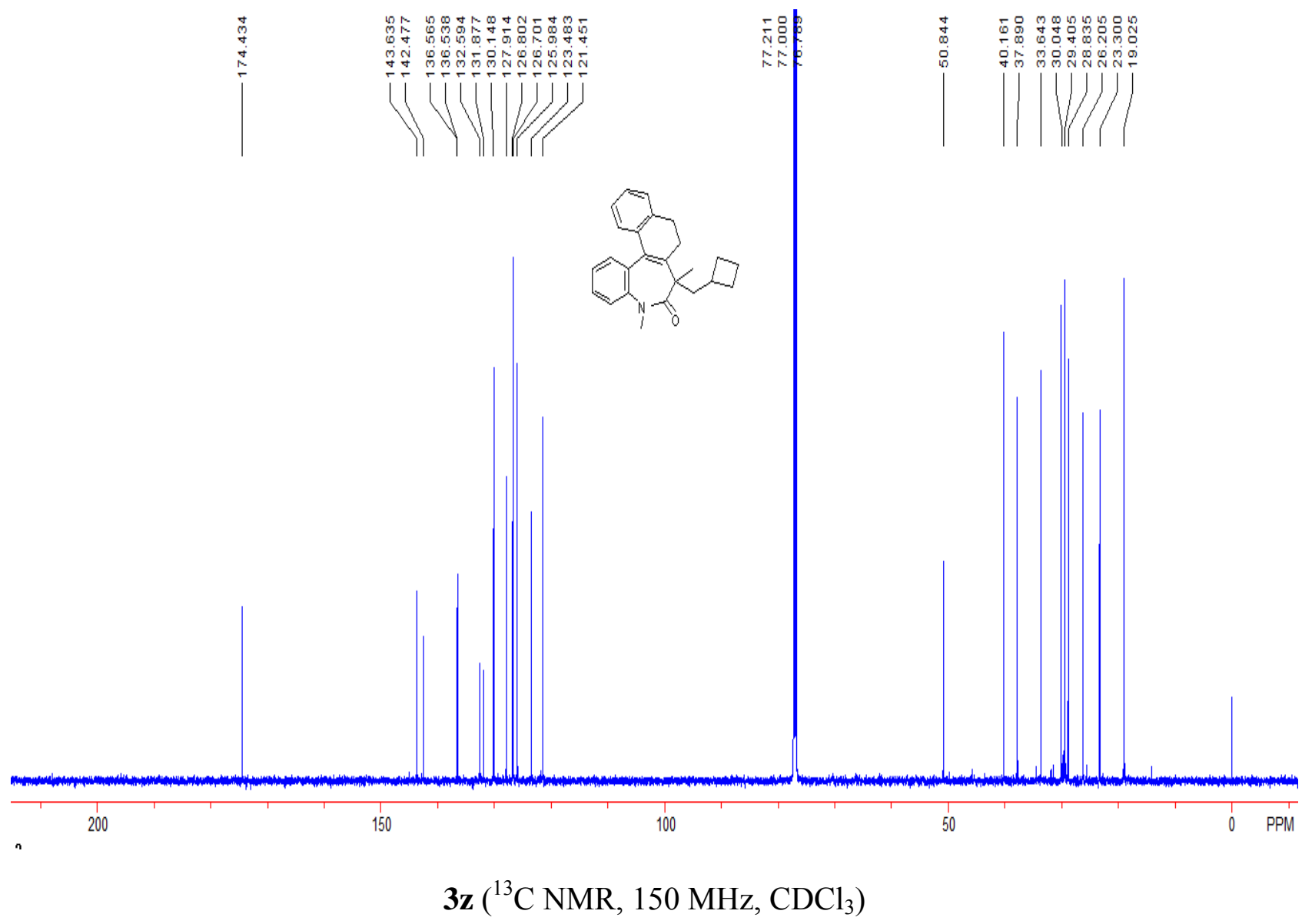


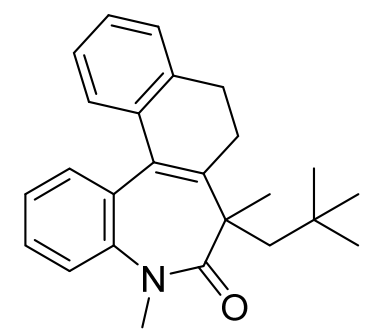

\section{5,7-Dimethyl-7-neopentyl-5,7,8,9-tetrahydro-6H-benzo[b]naphtho[1,2-d]azepin-6-one (3ab)}

Pale green oil, $50 \mathrm{mg}, 70 \%$ yield; Eluent: $\mathrm{PE} / \mathrm{EA}=10 / 1 .{ }^{1} \mathrm{H} \mathrm{NMR}\left(600 \mathrm{MHz}, \mathrm{CDCl}_{3}, \mathrm{TMS}\right) \delta$ 7.37-7.35 (m, 1H), 7.28-7.24 (m, 2H), 7.18-7.17 (m, 1H), 7.15-7.07 (m, 3H), $6.94(\mathrm{~d}, J=7.8 \mathrm{~Hz}$, $1 \mathrm{H}), 3.41(\mathrm{~s}, 3 \mathrm{H}), 2.85-2.74(\mathrm{~m}, 3 \mathrm{H}), 2.27\left(\mathrm{td}, J_{1}=6.0 \mathrm{~Hz}, J_{2}=16.4 \mathrm{~Hz}, 1 \mathrm{H}\right), 1.63(\mathrm{~s}, 3 \mathrm{H})$, 1.26-1.25 (m, 2H), $0.72(\mathrm{~s}, 9 \mathrm{H}) ;{ }^{13} \mathrm{C} \mathrm{NMR}\left(150 \mathrm{MHz}, \mathrm{CDCl}_{3}, \mathrm{TMS}\right) \delta 174.5,144.8,142.6,136.8$, $136.5,132.9,131.8,130.1,127.8,126.9,126.7,126.6,126.0,123.5,121.4,51.6,44.5,38.2,31.6$, 31.1, 29.1, 26.3, 24.4; IR $\left(\mathrm{CH}_{2} \mathrm{Cl}_{2}\right):$ v 2959, 2947, 2910, 1647, 1597, 1495, 1447, 1351, 1255, 849, 812, 767, 749, 729, $669 \mathrm{~cm}^{-1}$; HRMS (ESI) m/z: $(\mathrm{M}+\mathrm{H})^{+}$Calcd. for $\mathrm{C}_{25} \mathrm{H}_{30} \mathrm{NO} 360.2322$; found 360.2313 . 


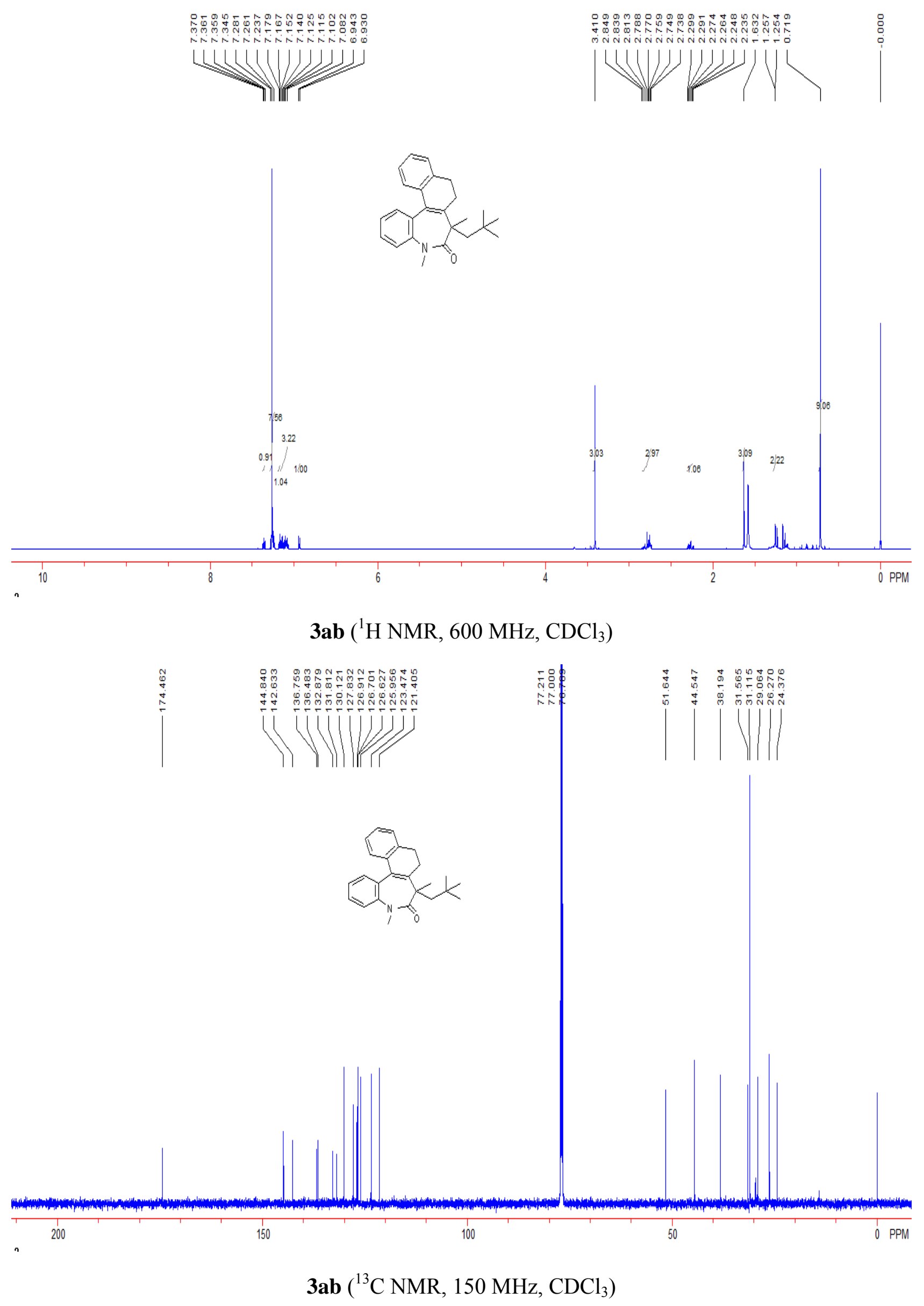




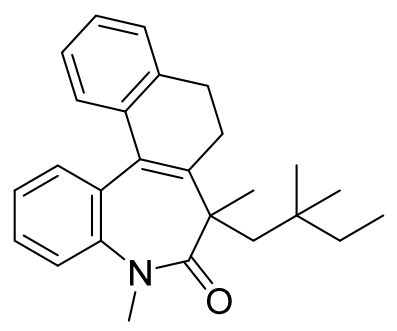

5,7-Dimethyl-7-(2,2-dimethylbutyl)-5,7,8,9-tetrahydro-6H-benzo[b]naphtho[1,2-d]azepin-6-on e (3ac)

Pale green oil, $50 \mathrm{mg}$, 67\% yield; Eluent: PE/EA = 10/1. ${ }^{1} \mathrm{H}$ NMR (400 $\left.\mathrm{MHz}, \mathrm{CDCl}_{3}, \mathrm{TMS}\right) \delta$ 7.38-7.33 (m, 1H), 7.29-7.23 (m, 2H), 7.18-7.06 (m, 4H), $6.93(\mathrm{~d}, J=7.6 \mathrm{~Hz}, 1 \mathrm{H}), 3.41(\mathrm{~s}, 3 \mathrm{H})$, 2.88-2.72 (m, 3H), 2.32-2.22 (m, 1H), $1.63(\mathrm{~s}, 3 \mathrm{H}), 1.21-1.19(\mathrm{~m}, 2 \mathrm{H}), 0.96-0.87$ (m, 2H), $0.75(\mathrm{~s}$, $3 \mathrm{H}), 0.68(\mathrm{~s}, 3 \mathrm{H}), 0.50(\mathrm{t}, J=7.6 \mathrm{~Hz}, 3 \mathrm{H}) ;{ }^{13} \mathrm{C} \mathrm{NMR}\left(150 \mathrm{MHz}, \mathrm{CDCl}_{3}, \mathrm{TMS}\right) \delta 174.6,145.0$, $142.7,136.8,136.5,132.9,131.8,130.2,127.9,126.9,126.7,126.6,126.0,123.5,121.4,51.6,42.2$, 38.2, 37.4, 34.1, 29.1, 27.7, 26.8, 26.3, 24.3, 8.1; IR $\left(\mathrm{CH}_{2} \mathrm{Cl}_{2}\right):$ v 2954, 2931, 1647, 1607, 1489, 1409, 1309, 1256, 1106, 1034, 849, 829, 777, 739, 709, $668 \mathrm{~cm}^{-1}$; HRMS (ESI) m/z: (M+H) Calcd. for $\mathrm{C}_{26} \mathrm{H}_{32} \mathrm{NO} 374.2478$; found 374.2471. 


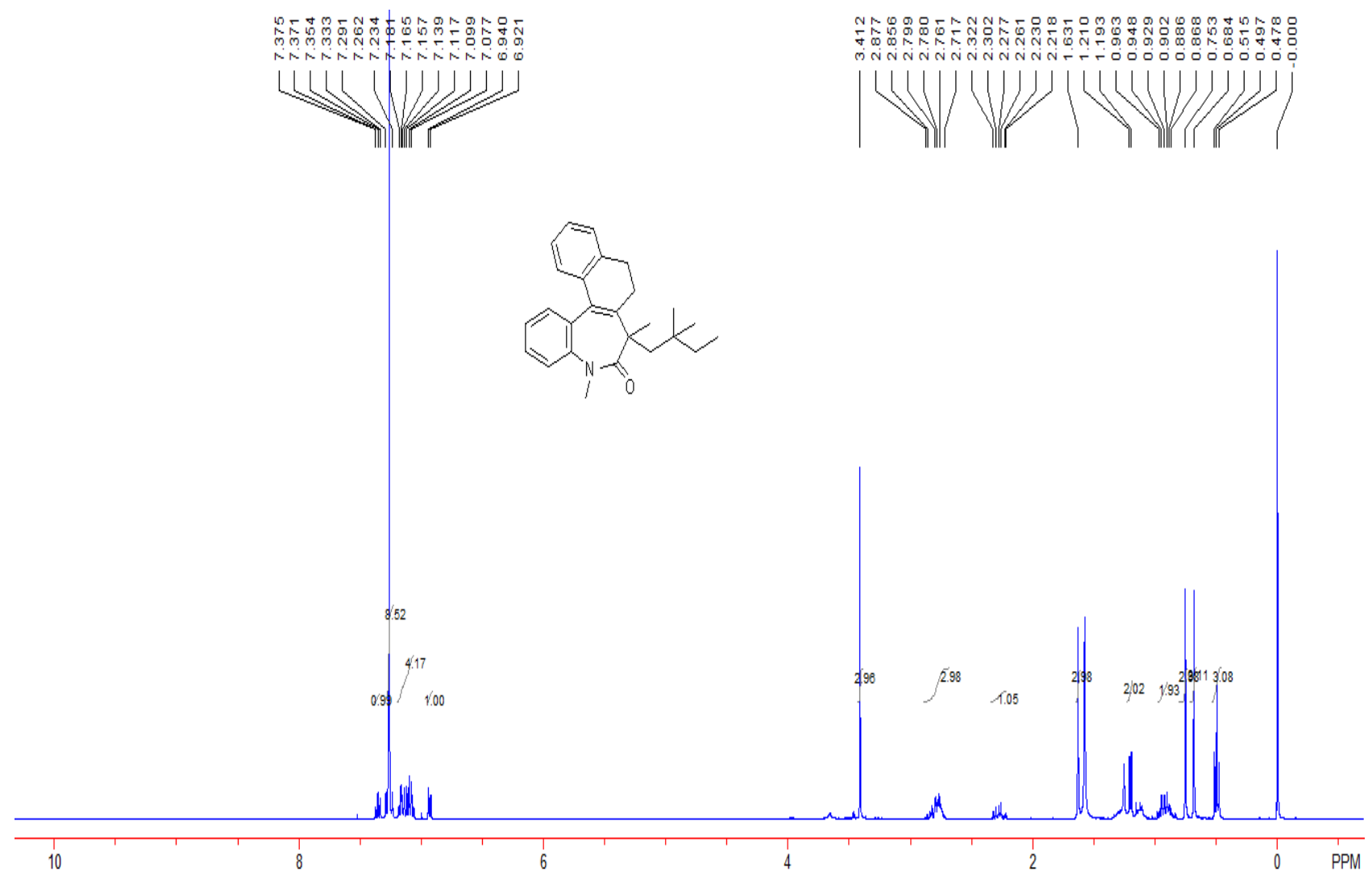

3ac $\left({ }^{1} \mathrm{H} \mathrm{NMR}, 400 \mathrm{MHz}, \mathrm{CDCl}_{3}\right)$
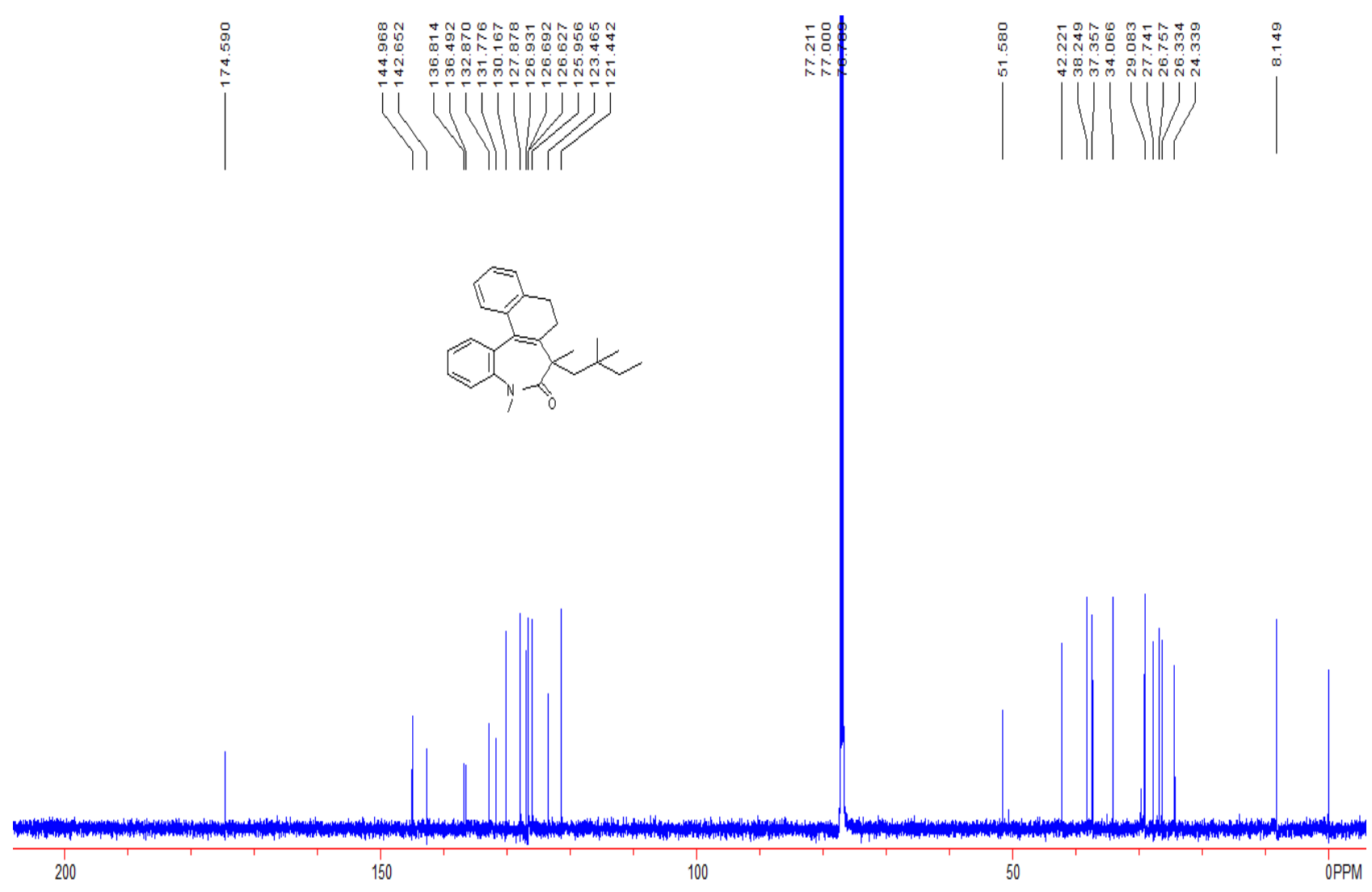

3ac $\left({ }^{13} \mathrm{C} \mathrm{NMR,} 150 \mathrm{MHz}, \mathrm{CDCl}_{3}\right)$ 


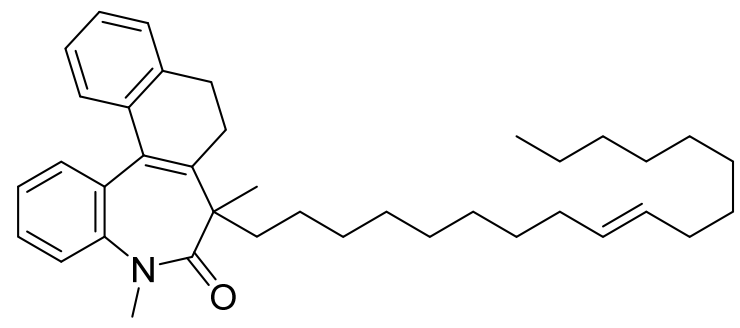

(E)-5,7-dimethyl-7-(octadec-9-en-1-yl)-5,7,8,9-tetrahydro-6H-benzo[b]naphtho[1,2-d]azepin-6one (3ad)

Pale green oil, $56 \mathrm{mg}, 75 \%$ yield; Eluent: PE/EA = 10/1. ${ }^{1} \mathrm{H}$ NMR (400 $\left.\mathrm{MHz}, \mathrm{CDCl}_{3}, \mathrm{TMS}\right) \delta$ 7.38-7.32 (m, 1H), 7.30-7.23 (m, 2H), 7.20-7.08 (m, 4H), 6.96 (d, J = 7.2 Hz, 1H), 5.38-5.35 (m, 2H), $3.41(\mathrm{~s}, 3 \mathrm{H}), 2.83-2.69(\mathrm{~m}, 3 \mathrm{H}), 2.24\left(\mathrm{td}, J_{1}=6.0 \mathrm{~Hz}, J_{2}=16.4 \mathrm{~Hz}, 1 \mathrm{H}\right), 1.96-1.92(\mathrm{~m}, 2 \mathrm{H})$, $1.51(\mathrm{~s}, 3 \mathrm{H}), 1.33-1.04(\mathrm{~m}, 25 \mathrm{H}), 0.97-0.79(\mathrm{~m}, 8 \mathrm{H}) ;{ }^{13} \mathrm{C} \mathrm{NMR}\left(150 \mathrm{MHz}, \mathrm{CDCl}_{3}, \mathrm{TMS}\right) \delta 174.5$, $143.8,142.3,136.6,136.5,132.6,131.8,130.4,130.3,130.0,127.9,126.8,126.73,126.70,126.0$, $123.5,121.4,50.9,37.7,34.1,32.6,31.9,29.64,29.55,29.5,29.29,29.28,29.2,29.0,28.7,26.2$, 25.4, 23.3, 22.7, 14.1; IR $\left(\mathrm{CH}_{2} \mathrm{Cl}_{2}\right)$ : v 2974, 2943, 2910, 2906, 1670, 1648, 1623, 1486, 1443, 1379, 1265, 1072, 1049, 1011, 977, 825, 808, 767, 747, 729, $669 \mathrm{~cm}^{-1}$; HRMS (ESI) m/z: (M+H) Calcd. for $\mathrm{C}_{38} \mathrm{H}_{54} \mathrm{NO} 540.4200$; found 540.4192 . 


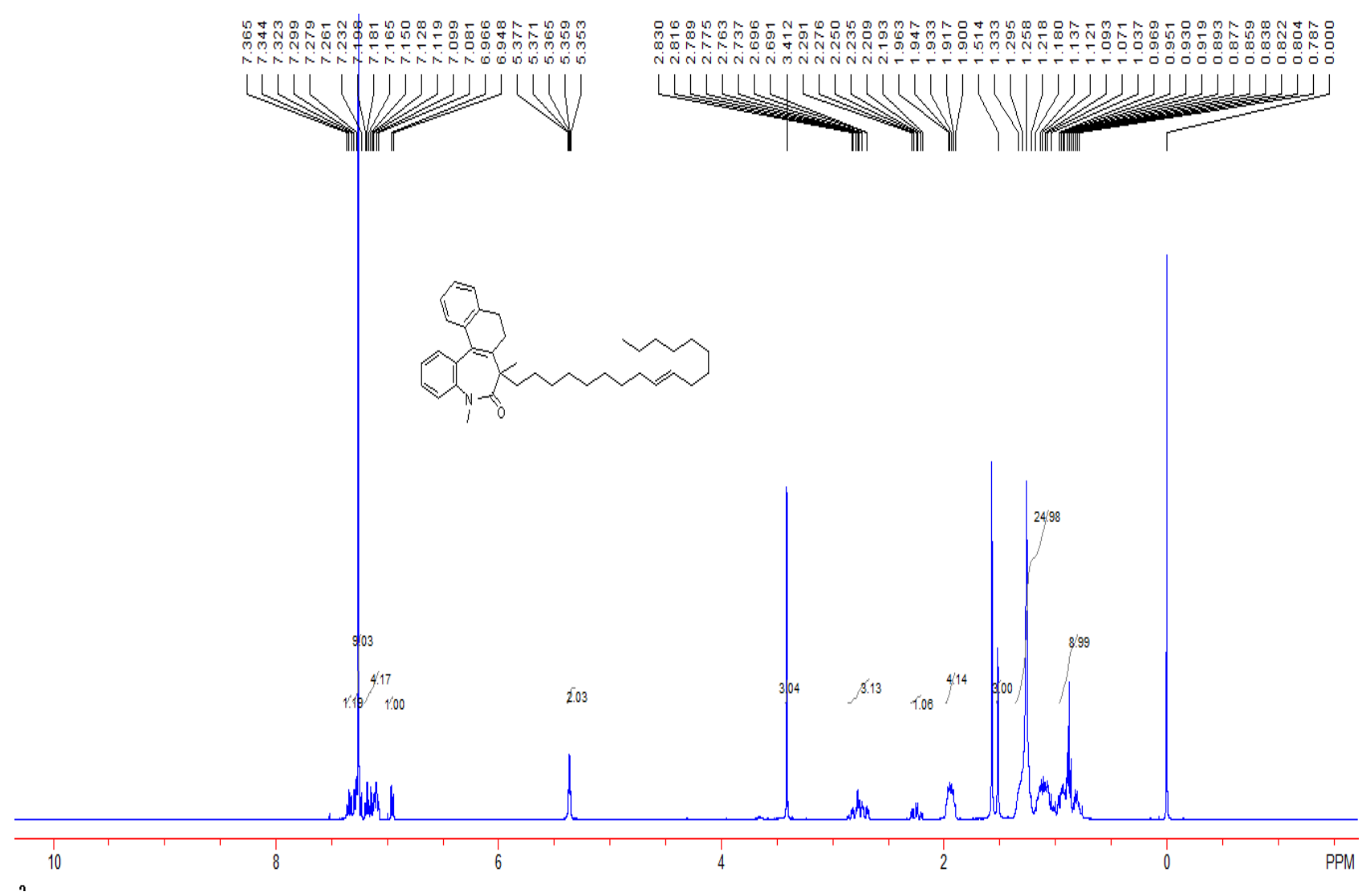

3ad $\left({ }^{1} \mathrm{H} \mathrm{NMR}, 400 \mathrm{MHz}, \mathrm{CDCl}_{3}\right)$

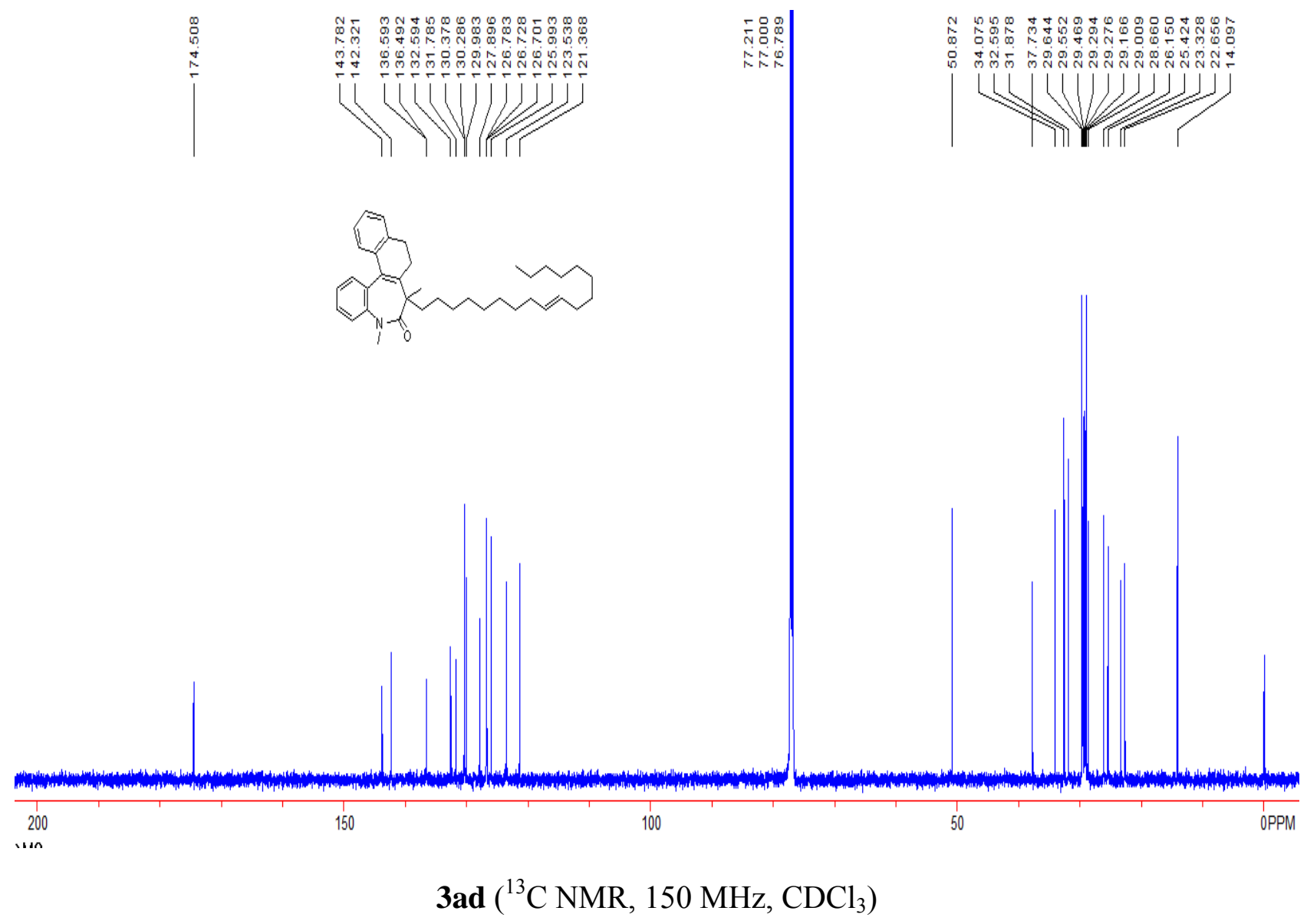




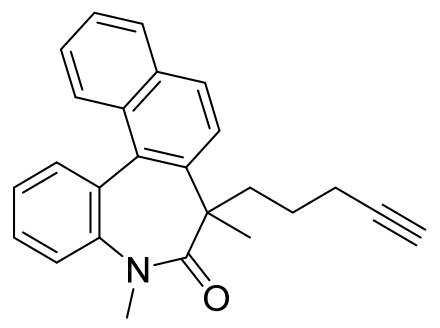

5,7-Dimethyl-7-(pent-4-yn-1-yl)-5,7-dihydro-6H-benzo[b]naphtho[1,2-d]azepin-6-one (4a)

Pale green oil, $69 \mathrm{mg}$, 98\% yield; Eluent: PE/EA = 10/1. ${ }^{1} \mathrm{H}$ NMR (400 MHz, $\mathrm{CDCl}_{3}$, TMS) $\delta 8.07$ $(\mathrm{d}, J=8.4 \mathrm{~Hz}, 1 \mathrm{H}), 7.87(\mathrm{~d}, J=9.2 \mathrm{~Hz}, 1 \mathrm{H}), 7.67(\mathrm{~d}, J=8.8 \mathrm{~Hz}, 1 \mathrm{H}), 7.58\left(\mathrm{dd}, J_{1}=1.6 \mathrm{~Hz}, J_{2}=8.0\right.$ $\mathrm{Hz}, 1 \mathrm{H}), 7.49-7.42(\mathrm{~m}, 3 \mathrm{H}), 7.38\left(\mathrm{dd}, J_{1}=0.8 \mathrm{~Hz}, J_{2}=8.0 \mathrm{~Hz}, 1 \mathrm{H}\right), 7.29-7.27(\mathrm{~m}, 1 \mathrm{H}), 3.37$ (s, 3H), $1.88(\mathrm{~s}, 3 \mathrm{H}), 1.75(\mathrm{t}, J=2.8 \mathrm{~Hz}, 1 \mathrm{H}), 1.70-1.68(\mathrm{~m}, 2 \mathrm{H}), 1.26-1.14(\mathrm{~m}, 4 \mathrm{H}) ;{ }^{13} \mathrm{C} \mathrm{NMR}(100 \mathrm{MHz}$, $\left.\mathrm{CDCl}_{3}, \mathrm{TMS}\right) \delta 175.0,142.0,140.7,133.2,132.9,132.8,132.3,131.6,128.7,128.4,127.9,126.7$, 126.2, 125.8, 124.0, 123.7, 121.9, 83.7, 68.5, 50.6, 37.5, 33.8, 25.1, 24.3, 18.6; IR $\left(\mathrm{CH}_{2} \mathrm{Cl}_{2}\right): v 3327$, 2938, 2922, 2200, 1648, 1491, 1443, 1347, 1091, 1015, 850, 777, 763, 753, 679, $667 \mathrm{~cm}^{-1}$; HRMS (ESI) $\mathrm{m} / \mathrm{z}:(\mathrm{M}+\mathrm{H})^{+}$Calcd. for $\mathrm{C}_{25} \mathrm{H}_{24} \mathrm{NO} 354.1852$; found 354.1848 . 


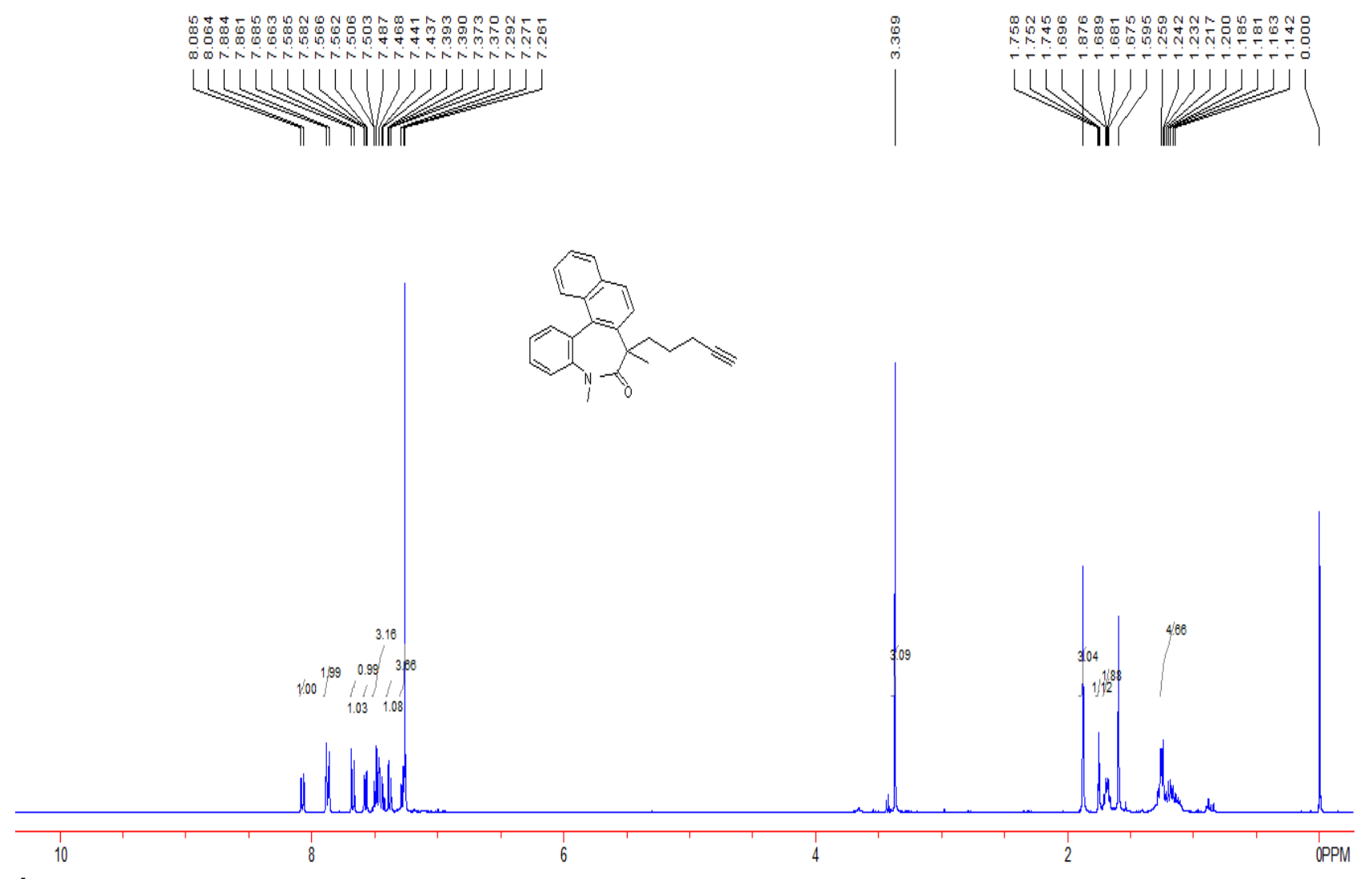

4a $\left({ }^{1} \mathrm{H}\right.$ NMR, $\left.400 \mathrm{MHz}, \mathrm{CDCl}_{3}\right)$
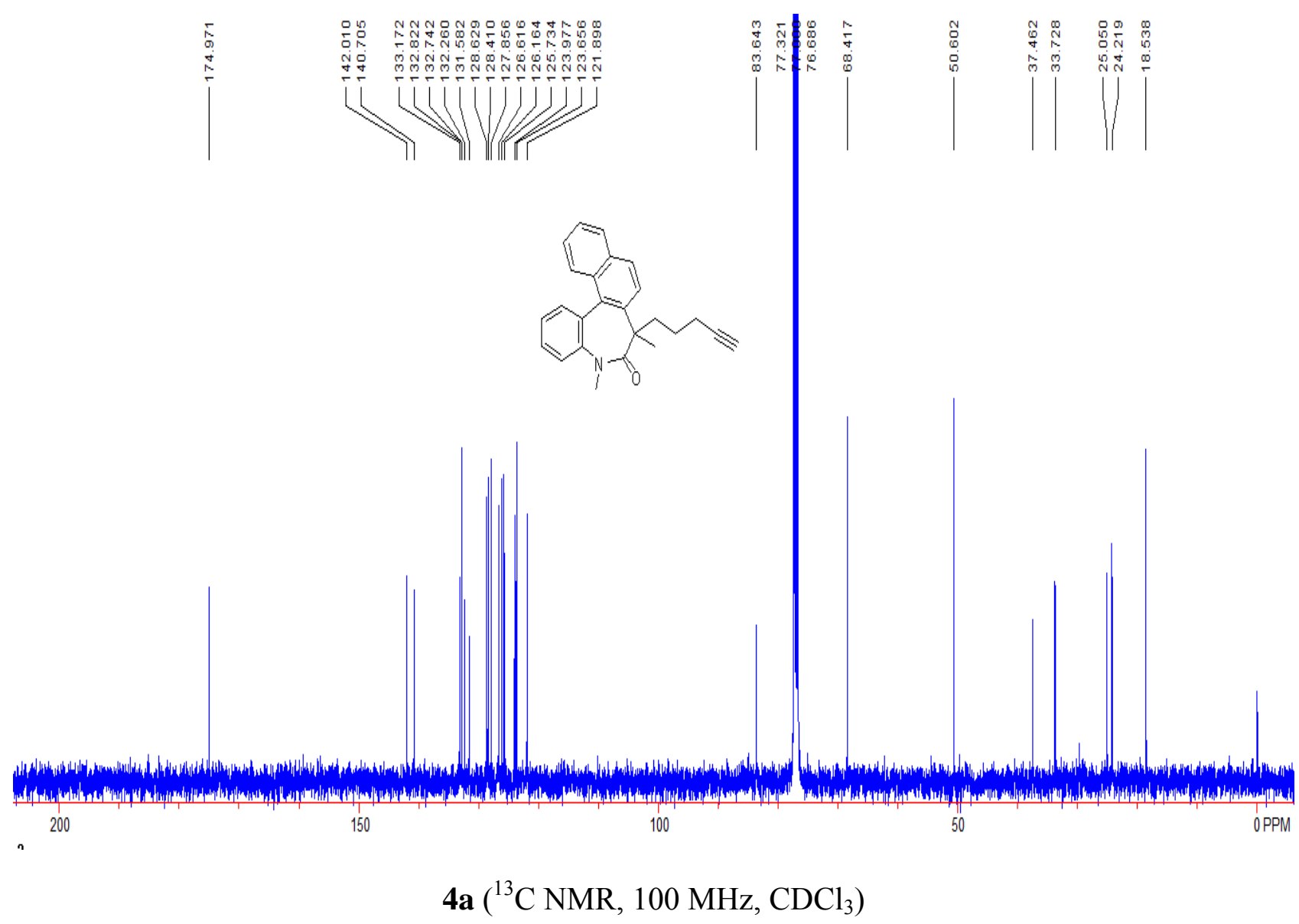


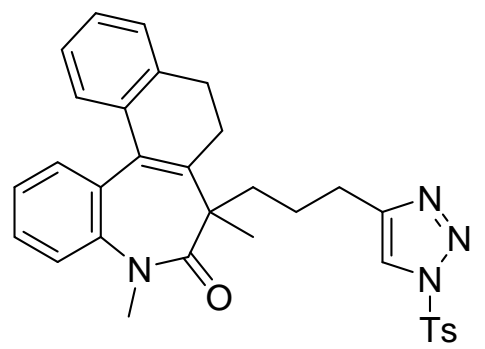

5,7-Dimethyl-7-(3-(1-tosyl-1H-1,2,3-triazol-4-yl)propyl)-5,7,8,9-tetrahydro-6H-benzo[b]napht ho[1,2-d]azepin-6-one (4b)

Pale green oil, $108 \mathrm{mg}$, 95\% yield; Eluent: PE/EA = 10/1. ${ }^{1} \mathrm{H}$ NMR (400 MHz, $\mathrm{CDCl}_{3}$, TMS) $\delta 7.95$ (d, $J=8.4 \mathrm{~Hz}, 2 \mathrm{H}), 7.55$ (s, 1H), 7.37 (d, $J=8.4 \mathrm{~Hz}, 2 \mathrm{H}), 7.34-7.30$ (m, 1H), 7.23-7.20 (m, 2H), 7.18-7.13 (m, 2H), 7.10-7.05 (m, 2H), $6.92(\mathrm{~d}, J=7.6 \mathrm{~Hz}, 1 \mathrm{H}), 3.39$ (s, 3H), 2.86-2.65 (m, 3H), $2.44(\mathrm{~s}, 3 \mathrm{H}), 2.30-2.20(\mathrm{~m}, 3 \mathrm{H}), 1.51(\mathrm{~s}, 3 \mathrm{H}), 1.33-1.26(\mathrm{~m}, 2 \mathrm{H}), 1.13-1.02(\mathrm{~m}, 2 \mathrm{H}) ;{ }^{13} \mathrm{C}$ NMR $(150$ $\mathrm{MHz}_{2} \mathrm{CDCl}_{3}$, TMS) $\delta 173.9,147.08,147.07,142.9,142.0,136.5,136.2,133.3,132.2,132.1,130.4$, $129.8,128.5,128.2,126.84,126.79,126.8,126.0,123.8,121.2,120.2,50.5,37.7,33.5,28.6,26.0$, 25.4, 25.0, 23.3, 21.8; IR $\left(\mathrm{CH}_{2} \mathrm{Cl}_{2}\right):$ v 2924, 1641, 1595, 1389, 1193, 1178, 1092, 1009, 969, 768, 730, $669 \mathrm{~cm}^{-1}$; HRMS (ESI) m/z: (M+H) ${ }^{+}$Calcd. for $\mathrm{C}_{32} \mathrm{H}_{33} \mathrm{~N}_{4} \mathrm{O}_{3} \mathrm{~S} 553.2268$; found 553.2250. 

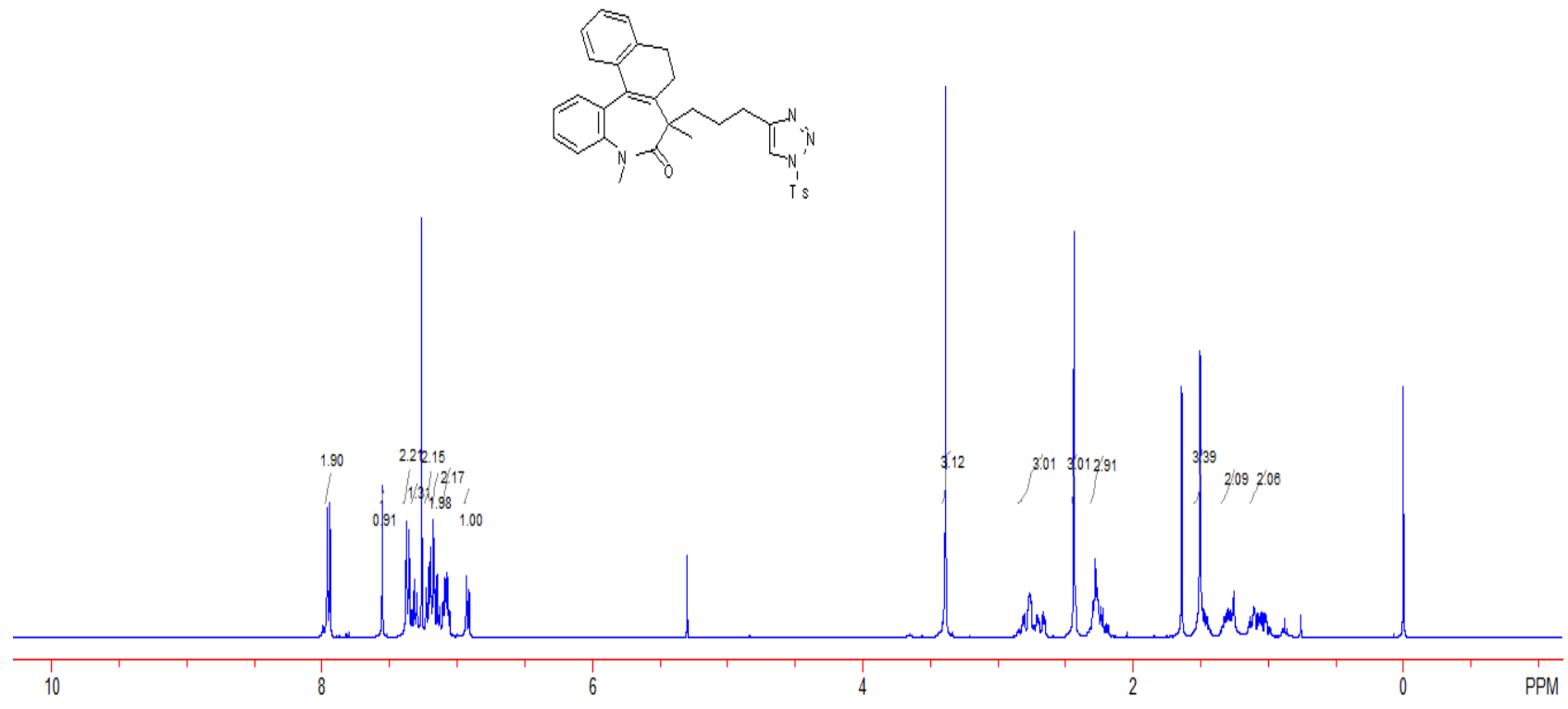

4b $\left({ }^{1} \mathrm{H}\right.$ NMR, $\left.400 \mathrm{MHz}, \mathrm{CDCl}_{3}\right)$
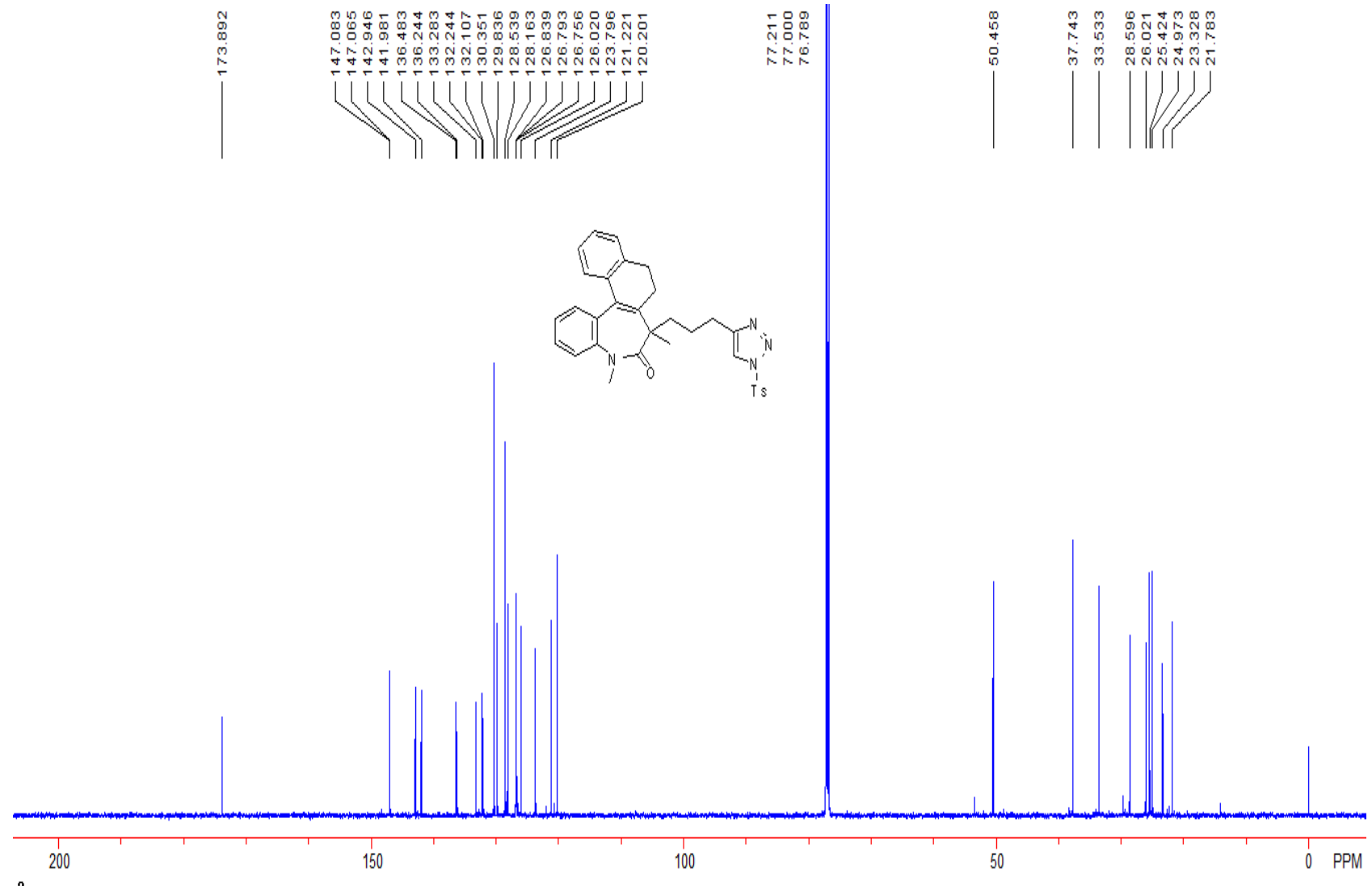

4b $\left({ }^{13} \mathrm{C} \mathrm{NMR}, 150 \mathrm{MHz}, \mathrm{CDCl}_{3}\right)$ 


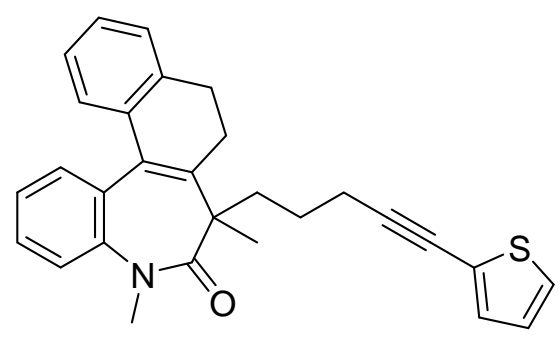

5,7-Dimethyl-7-(5-(thiophen-2-yl)pent-4-yn-1-yl)-5,7,8,9-tetrahydro-6H-benzo[b]naphtho[1,2d]azepin-6-one (4c)

Pale green oil, $75 \mathrm{mg}, 98 \%$ yield; Eluent: PE/EA $=10 / 1 .{ }^{1} \mathrm{H}$ NMR (400 $\left.\mathrm{MHz}, \mathrm{CDCl}_{3}, \mathrm{TMS}\right) \delta$ 7.30-7.27 (m, 3H), 7.20-7.13 (m, 3H), 7.11-7.07 (m, 1H), 7.04-7.01 (m, 2H), 6.96-6.91 (m, 2H), $3.42(\mathrm{~s}, 3 \mathrm{H}), 2.84-2.70(\mathrm{~m}, 3 \mathrm{H}), 2.26\left(\mathrm{td}, J_{1}=6.4 \mathrm{~Hz}, J_{2}=16.4 \mathrm{~Hz}, 1 \mathrm{H}\right), 2.00-1.97(\mathrm{~m}, 2 \mathrm{H}), 1.56(\mathrm{~s}$, 3H), 1.30-1.13 (m, 4H); ${ }^{13} \mathrm{C}$ NMR (150 MHz, $\mathrm{CDCl}_{3}$, TMS) $\delta 174.0,143.1,142.1,136.5,136.3$, 132.3, 132.1, 131.0, 130.0, 128.1, 126.81, 126.77, 126.7, 126.0, 125.9, 124.0, 123.8, 121.3, 93.4, 73.8, 50.5, 37.8, 33.7, 28.6, 26.1, 24.8, 23.4, 19.9; IR $\left(\mathrm{CH}_{2} \mathrm{Cl}_{2}\right): v 2974,2932,2201,1645,1494$, 1446, 1441, 1354, 1217, 1097, 1048, 881, 777, 762, 754, 672, $657 \mathrm{~cm}^{-1}$; HRMS (ESI) m/z: (M+H) Calcd. for $\mathrm{C}_{29} \mathrm{H}_{28} \mathrm{NOS}$ 438.1886; found 438.1882. 


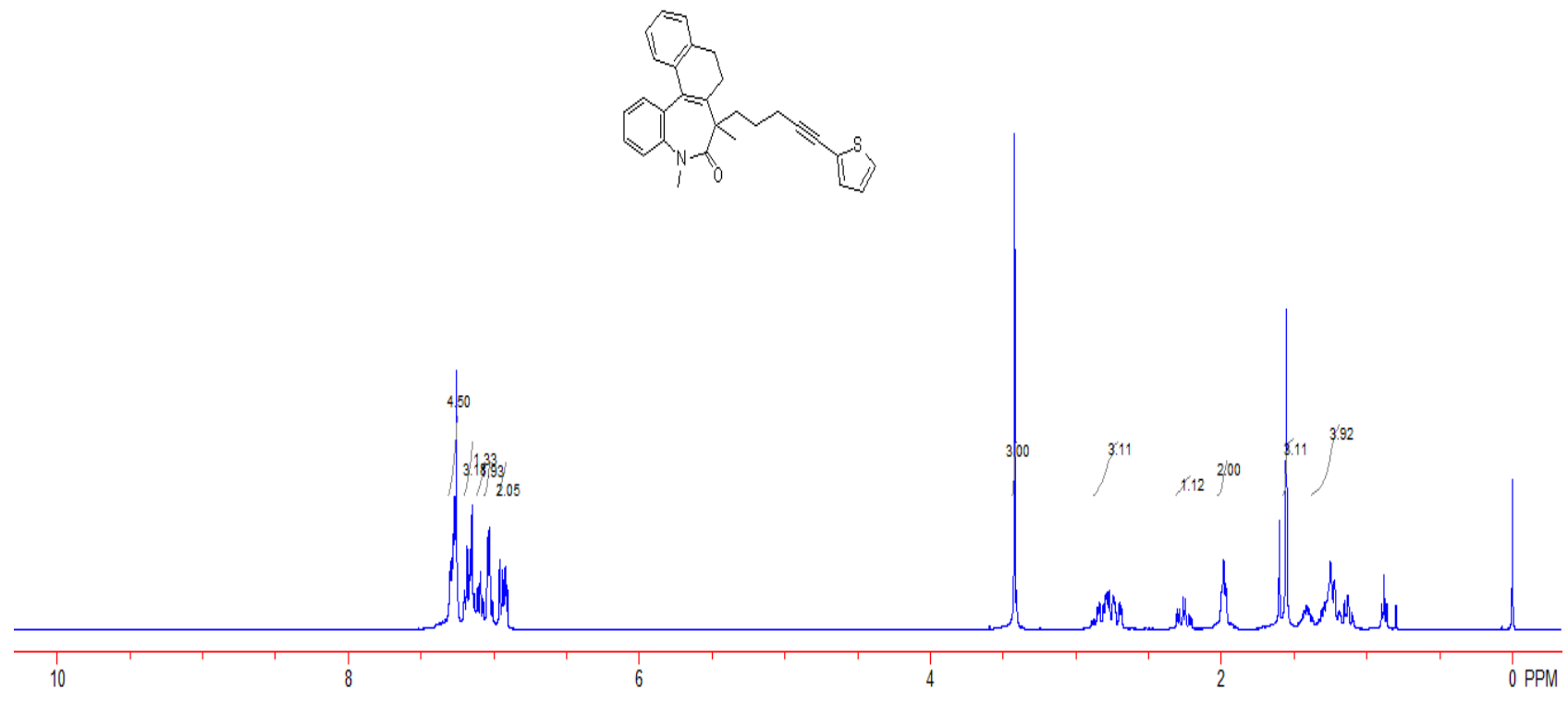

4c $\left({ }^{1} \mathrm{H}\right.$ NMR, $\left.400 \mathrm{MHz}, \mathrm{CDCl}_{3}\right)$

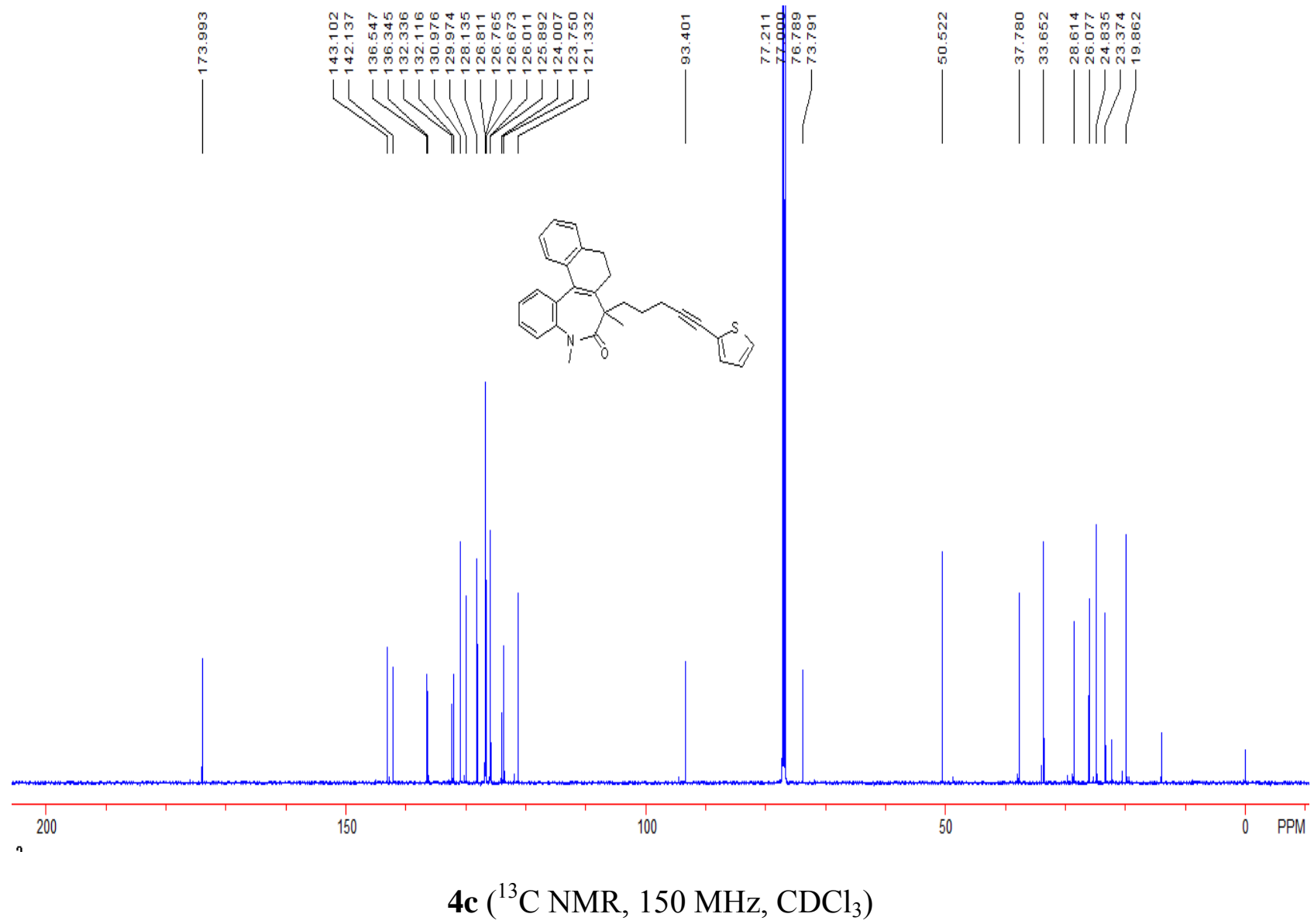




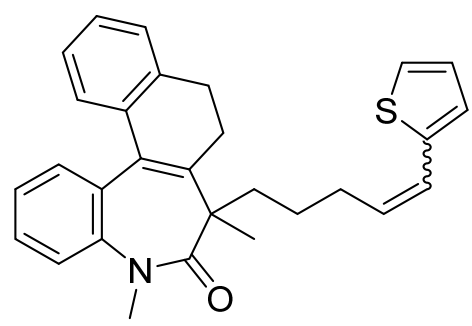

5,7-Dimethyl-7-(5-(thiophen-2-yl)pent-4-en-1-yl)-5,7,8,9-tetrahydro-6H-benzo[b]naphtho[1,2d]azepin-6-one (4d)

Pale green oil, $74 \mathrm{mg}, Z: E=5: 1,98 \%$ yield; Eluent: PE/EA $=10 / 1 .{ }^{1} \mathrm{H} \mathrm{NMR}\left(400 \mathrm{MHz}, \mathrm{CDCl}_{3}\right.$, TMS) $\delta$ 7.37-7.31 (m, 1.22), 7.29-7.24 (m, 2.4H), 7.20-7.08 (m, 6H), 6.96-6.90 (m, 2.43), 6.85-6.78 $(\mathrm{m}, 1.2 \mathrm{H}), 6.33(\mathrm{~d}, J=11.2 \mathrm{~Hz}, 1 \mathrm{H}), 6.24(\mathrm{~d}, J=15.6 \mathrm{~Hz}, 0.2 \mathrm{H}), 5.70-5.61(\mathrm{~m}, 0.2 \mathrm{H}), 5.12\left(\mathrm{dt}, J_{1}=\right.$ $\left.7.2 \mathrm{~Hz}, J_{2}=11.6 \mathrm{~Hz}, 1 \mathrm{H}\right), 3.42(\mathrm{~s}, 3.63 \mathrm{H}), 2.86-2.69(\mathrm{~m}, 3.59 \mathrm{H}), 2.30-2.20(\mathrm{~m}, 1.21 \mathrm{H}), 2.00-1.96$ (m, 2.37H), $1.54(\mathrm{~s}, 3.68 \mathrm{H}), 1.20-1.09(\mathrm{~m}, 5.49 \mathrm{H}) ;{ }^{13} \mathrm{C} \mathrm{NMR}\left(150 \mathrm{MHz}, \mathrm{CDCl}_{3}, \mathrm{TMS}\right) \delta 174.3$, $174.2,143.4,142.8,142.3,142.2$, 140.5, 136.58, 136.56, 136.43, 136.40, 132.5, 132.4, 132.0, 130.2, $130.1,130.0,129.9,128.0,127.1,127.0,126.80,126.77,126.6,126.0,124.8,124.2,123.7,123.6$, $123.3,123.1,121.9,121.5,121.3,50.74,50.70,37.80,37.75,33.7,32.9,29.7,29.1,28.6,26.13$, 26.10, 25.4, 25.2, 23.4; IR $\left(\mathrm{CH}_{2} \mathrm{Cl}_{2}\right):$ v 2932, 1642, 1589, 1394, 1178, 1093, 1020, 988, 969, 778, 728, $668 \mathrm{~cm}^{-1}$; HRMS (ESI) m/z: (M+H) ${ }^{+}$Calcd. for $\mathrm{C}_{29} \mathrm{H}_{30} \mathrm{NOS} 440.2043$; found 440.2032. 


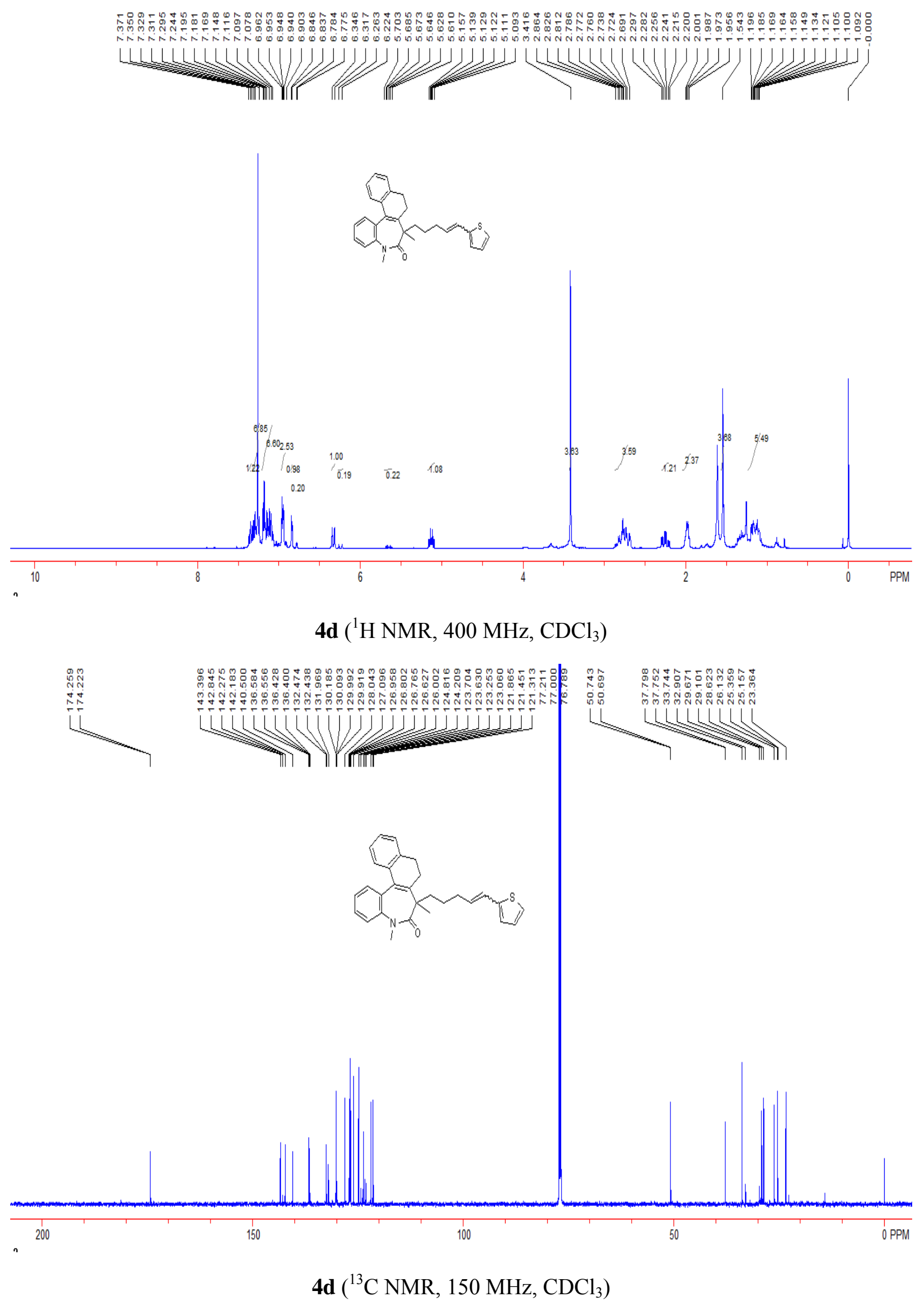




\section{X-ray data.}

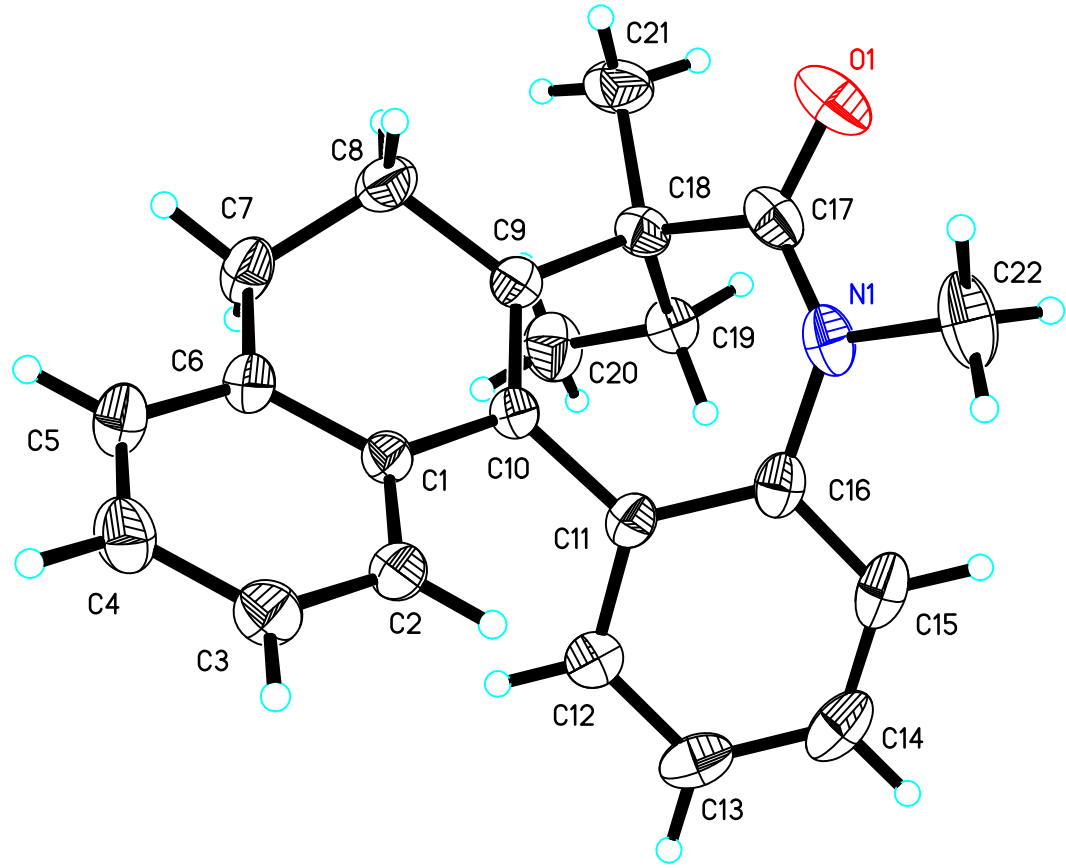

Single crystals of 3a were grown in dichloromethane and hexanes. Dichloromethane $(2.0 \mathrm{~mL})$ was added to 3a (15 mg in a $4 \mathrm{~mL}$ vial) followed by 3 drops of hexanes. The $4 \mathrm{~mL}$ vial was capped and placed at room temperature in the experimental cabinet for 3 days, whereupon crystals formed. A colorless block shaped crystal of 3a was used for the X-ray crystallographic analysis. The X-ray intensity data were measured at 293(2) K, on a Rigaku AFC7R diffractometer. The crystal data of 3a have been deposited in CCDC with number 1937021 and have been displayed at 30\% ellipsoid contour probability level.

Table 1. Crystal data and structure refinement for 3a.

Identification code

Empirical formula

Formula weight

Temperature

Wavelength

Crystal system 3a

$\mathrm{C}_{22} \mathrm{H}_{23} \mathrm{NO}$

317.41

293(2) K

$0.71073 \AA$

Monoclinic 
Space group

Unit cell dimensions

Volume

Z

Density (calculated)

Absorption coefficient

$\mathrm{F}(000)$

Crystal size

Theta range for data collection

Index ranges

Reflections collected

Independent reflections

Completeness to theta $=25.242^{\circ}$

Absorption correction

Max. and min. transmission

Refinement method

Data / restraints / parameters

Goodness-of-fit on $\mathrm{F}^{2}$

Final $\mathrm{R}$ indices $[\mathrm{I}>2 \operatorname{sigma}(\mathrm{I})]$

$\mathrm{R}$ indices (all data)

Extinction coefficient

Largest diff. peak and hole
P 21/c

$$
\begin{array}{ll}
\mathrm{a}=7.8709(4) \AA & \mathrm{a}=90^{\circ} . \\
\mathrm{b}=17.9762(7) \AA & \mathrm{b}=102.141(2)^{\circ} . \\
\mathrm{c}=12.3763(6) \AA & \mathrm{g}=90^{\circ} .
\end{array}
$$

1711.94(14) $\AA^{3}$

4

\section{$1.232 \mathrm{Mg} / \mathrm{m}^{3}$}

$0.075 \mathrm{~mm}^{-1}$

680

$0.200 \times 0.140 \times 0.110 \mathrm{~mm}^{3}$

2.266 to $25.999^{\circ}$.

$-6 \leq \mathrm{h} \leq 9,-22 \leq \mathrm{k} \leq 22,-15 \leq 1 \leq 14$

8496

$3346[\mathrm{R}($ int $)=0.0465]$

$99.4 \%$

Semi-empirical from equivalents

0.7456 and 0.5928

Full-matrix least-squares on $\mathrm{F}^{2}$

$3346 / 0 / 221$

1.024

$\mathrm{R} 1=0.0526, \mathrm{wR} 2=0.1248$

$\mathrm{R} 1=0.0817, \mathrm{wR} 2=0.1479$

$0.059(7)$

0.214 and -0.148 e. $\AA^{-3}$ 


\section{References.}

1) Yu, L.-Z.; Xu, Q.; Tang, X.-Y.; Shi. M. ACS Catalysis 2016, 6, 526.

2) Stang, P. J.; Boehshar, M.; Wingert, H.; Kitamura, T. J. Am. Chem. Soc. 1988, 110, 3272. 